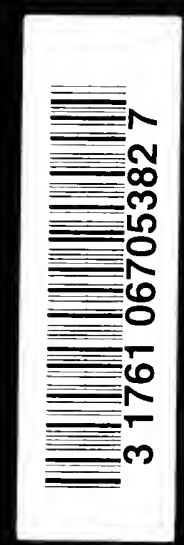




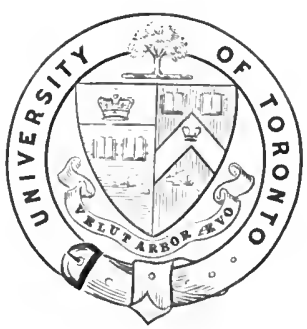

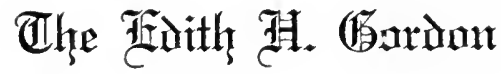
迎equest - 1940 in mtemoriant

Fititl 其:

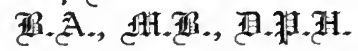

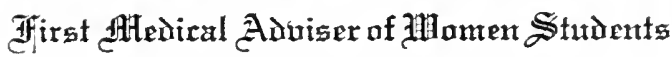
Hetniversity of $\mathbb{C}$ aranto Ffrom 1921 to 1939

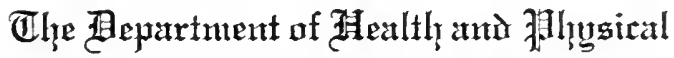
Touncation for 
MS

N

\section{Diseases and Deformities} $\mathrm{OF}$

\section{THE FOOT}

BY

JOHN JOSEPH NUTT, B.L., M.D. Surgeon-in-Chief, New York State Hospital for the Care of Crippled and Deformed Children; Surgeon, Sea Breeze Hospital; Assistant Attending Surgeon, in Charge of Orthopedic Cases, Willard Parker Hospital; Member of the American Orthopedic Association.

ILLUSTRATED

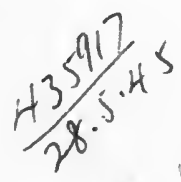

NEW YORK

E. B. TRE.AT \& COMPANY

24I-3 WEST 23D ST.

I 9 I 5 
Copyright, 1913

By E. B. TREAT \& Co. 


\section{PREFACE}

This handbook is prepared for the use of physicians who have not had the time or the opportunity for thorough study of this often neglected subject and who feel keenly their inability to prescribe scientifically and successfully for the many who consult them regarding their pedal conditions. Textbooks on orthopedic surgery are rarely consulted by the general practitioner, as most of the diseases and deformities of the frame-work of the body demand such treatment as only orthopedic surgeons are prepared to give.

With regard to the feet, however, much of the treatment is so simple that the general practitioner can and should assume the responsibility of preventing deformities, correcting abuses and those conditions which have already occurred and treating minor diseases of the bones and joints. Many painful and disagreeable conditions, such as chilblains, corns, ingrowing toe-nail, painful heel, excessive sweating of the feet, etc., may be cured by simple measures, and these, as well as the operations for severer complications, are herein fully described and amply illustrated.

2020 Broadway, New York.

John Joseph Nutt, M. D.

September 10, I9I3. 
Digitized by the Internet Archive in 2007 with funding from Microsoft Corporation 


\title{
CONTENTS
}

\author{
CHAPTER I
}

ANATOMY

PAGE

Skeleton of the Foot - Bones - Ligaments - Muscles and Tendons - Nerves . . . . . . . . . . 13

\section{CHAPTER II \\ PHYSIOLOGY}

Distribution of Weight - Movements - The Foot at Rest - Standing - Work, Rest, Fatigue — Walking . . . . . . . . . . . . . . $4 \mathrm{I}$

CHAPTER III

EXAMINATION

Inspection - History - Palpation - Pain . . . . 59

CHAPTER IV

SHAFFER'S FOOT. WEAK-FOOT.. FLAT-FOOT

Shaffer's Foot - Consequences - Symptoms - Etiology - Treatment - Traction Shoes - Operation - Weak-Foot - Characteristics - Results of Non-Treatment - Treatment - Flat-Foot - Osseous Flat-Foot - Pathology - Treatment of Weak-Foot and Flat-Foot-Exercises - Arch Supporters - Whitman's Brace - Shaffer's Brace - Manipulations - Strapping-Severe Cases Plaster-of-Paris Dressing - Tenotomy -Excision 
- Arthrodesis - Tarsectomy - Scaphoidectomy

-Post-Operative Treatment-Liniments . . 64

CHAPTER V

\section{CONGENITAL CLUB-FOOT}

Varieties - Frequency - Etiology - General Appearance - Explanation of Appearance - Changes in the Bones - Changes in Ligaments and Muscles - Pathology After Use - Confirmation of Structural Changes . . . . . . . . . III

\section{CHAPTER VI}

\section{TREATMENT OF CONGENITAL CLUB-FOOT}

Early Treatment - Manipulation - Muslin Bandage - Adhesive Plaster - Braces - Felt Splint Wood Splint - Plaster-of-Paris - Willard's Shoe - Taylor's Brace - Deformity in the Long Bones - Therapeutics of Early Treatment - Treatment When the Child Begins to Stand - Congenital Talipes Equino-Valgus - Congenital Talipes Equinus - Congenital Talipes Valgus - Congenital Talipes Varus - Congenital Talipes Calcaneous - Congenital Talipes Valgus and Varus - Congenital Talipes Planus - Congenital Talipes Cavus - Congenital Talipes Valgo-Cavus - Congenital Talipes EquinoCavus - Operative Procedures in the Treatment of Congenital Club-Foot - Wolff's Law - Davis' Law - Tenotomy - History - Value - Tendo Achillis - Posterior Ligament - Tibialis Posticus - Astragalo-Scaphoid Capsule - Flexor Longus Digitorum - Tibialis Anticus - Peroneus Longus and Brevis - Plantar Fascia - Danger of Aneurism - Indications for Tenotomy - Phelps' Operation - Bone Operations - Indications - Bones to be Attacked - Astragalectomy - Partial Excision - Ogston's Operation - Cuneiform Tarsectomy —Multiple Cuneiform Osteotamies . . . . I25 
CHAPTER VII

POTTS' PARAPLEGIA. CEREBRAL PARALYSIS

Paralysis Complicating Pott's Disease-Spastic Paralysis - Diagnosis - Examination - Treatment - Tenotomies - Neurotomy - Injections of Alcohol — Stoeffel's Technique . . . . . . . I78

\section{CHAPTER VIII}

\section{INFANTILE PARALYSIS}

Residual Paralysis - Diagnosis - Recent Cases Apparatus - Active Movements - Massage Electricity - Heat - Functional Use - Untreated Cases of Long Standing - Valgus - Varus Equinus - Calcaneus - Cavus - Hollow ClawFoot - Cases of Undoubtable Permanent Paralysis-Reduction of Deformity - Recovery of Muscles - Braces - Tendon Transplantation Astragalectomy . . . . . . . . . . 183

CHAPTER IX

\section{TUBERCULOSIS AND GONORRHEAL DISEASE}

Tuberculous Disease of the Foot-Diagnosis - Differential Diagnosis - Immobilization - Campbell Braces - Thomas Knee Brace - Bier's Treatment - Tuberculin Treatment - Heliotherapy - Seabathing - Constitutional Treatment - Operations - Sinuses - Injections - Gonorrheal Infection of the Foot - Treatment . . . . . . . . 217

CHAPTER X

OTHER AILMENTS

Painful Heel - Treatment - Operation - Metatarsalgia - Treatment - Morton's Toe - Treatment Hallux Valgus - Treatment - Operation - Ham- 


\section{CONTENTS}

mer-Toe - Treatment - Operation - Raynaud's Disease - Treatment - Myasthenia Angio-Solerotica (Intermittent Limping) - Treatment - Perforating Ulcer of the Foot - Treatment - Pernio (Chilblains) - Treatment - Congelation (FrostBite) - Treatment - Hyperidrosis (Excessive Sweating) - Treatment - Erythromelalgia Plantar Neuralgia - Clavus (Corns) - Treatment - Paronychia (Ingrowing Toe-Nail) - Treatment - Callosities - Painful Soles . . . 248

CHAPTER XI

FOOT APPAREL

Stockings - Shoes - Heels - Rocker Sole . . • 277 


\section{ILLUSTRATIONS}

PAGE

Plate I Radiograph of a Practically Normal Forefoot I6 II Unilateral Congenital Talipes Equino-Varus I20 III Flail Ankle Supported by Silk Ligaments . I84

“ IV Tuberculous Disease with Old Scars . . . 224 FIG.

I. Axis of Ankle-Joint Movement . . . . I4

2. Trochlear Surface of Astragalus . . . . . $\mathrm{I}_{5}$

3. Three Principal Axes of Movements of the Foot 25

4. Plantar Flexion in Walking . . . . . $3 \mathbf{I}$

5. Tibial Flexion • • • • • • . . . . 44

6. Movements at the Ankle-Joint . . . . . 45

7. Movements at the Sub-Astragaloid Joint . •, 46

8. Movements at the Medio-Tarsal Joint . . . 47

9. Standing Positions . . . . . . . . . 50

ı. Heel-Walking . . . . . . . . . . 54

I I. Toe-Walking . • • . . . . . . 56

12-I4. Effect of a Shortened Gastrocnemius . . . 65

15-16. Effect of a Shortened Gastrocnemius . . 67

17-18. Illustrating Difficulty in Descending Stairs when Dorsal Flexion of Foot is Limited . . . 69

19-20. Illustrating Difficulty in Descending Stairs when Dorsal Flexion of Foot is Limited . . 70 


\section{ILLUSTRATIONS}

FIG.

2I. Author's Traction Shoe . . . . . . . 73

22. Author's Traction Shoe in Detail . . . . 75

23. Lengthening the Tendo Achillis . . . . . 77

24-25. Adducted and Everted Feet . . . . . 79

26. Abducted and Everted Feet Seen from Behind . 80

27. Abducted and Everted Feet Seen from in Front 80

28-29. Advancement of the External Malleolus . . 8r

30-3r. A Shoe Before and After the Insertion of Wedges . . . . . . . . . 87

32-33. Inverting Heel . . . . . . . . . 88

34. An Exercise for Weak Feet . . . . . . 90

35. The Feet at Rest . . . . . . . . . $9 \mathrm{I}$

36. Forcible Correction of Abduction . . . 98

37-38. Adhesive Plaster Strapping . . . . . 99

39. Manipulation of Metatarsal Joints . . . . Ior

40. Manipulation of the Toe-Joints . . . . . IO2

4I-42. The Plaster-of-Paris Bandage . . . . . 104

43. Congenital Talipes Equino-Varus . . . . I2I

44. Manipulation of Congenital Club-Foot . . . I27

45-52. The Judson Club-Foot Brace . . . . . . I32

53. The Single Steel Bar Brace, First Position . . I34

54. The Single Steel Bar Brace, Second Position • I35

85. The Single Steel Bar Brace, Third Position . . 136

56. The Taylor Club-Foot Brace . . . . . . I4I

57. The Taylor Club-Foot Brace, Applied . . . I42

58. The Taylor Club-Foot Brace, Applied . . . 147 


\section{ILLUSTRATIONS}

FIG.

59. The Use of a Wedge in Adduction . . . . I55

6o. Relapsed Congenital Talipes Equino-Varus . . I59

6r. Dangle-Foot . . . . . . . . . . 184

62. Paralytic Talipes Valgus . . . . . . . I90

63-65. Examples of Equinus Deformity . . . . . I9I

66-68. Varieties of Calcaneus . . . . . . . 193

69. Paralytic Calcaneus . . . . . . . . . 194

70. Talipes Plantaris . . . . . . . . . 195

71. Talipes Arcuatus . . . . . . . . . 196

72. Paralysis of Anterior Muscles . . . . . 198

73. Paralysis of Tendo Achillis and Anterior Muscles . . . . . . . . . . 199

74. Paralysis of Posterior and Plantar Muscles . . 200

75. Paralysis of All Muscles Except Gastroc-

Nemius and Soleus . . . . . . . 201

76. Shaffer's Lateral Traction Shoe . . . . . 204

77. Right-Angle Stop at Ankle-Joint . . . . . 206

78. Reverse-Stop at Ankle-Joint . . . . . . 207

79. A Stop-Joint Allowing a Few Degrees of

Motion Only . . . . . . . . . 207

8o. Location of Ankle-Brace Joint . . . . . 208

81. Night-Shoe . . . . . . . . . . . 2II

82. Tuberculous Foot . . . . . . . . . 219

83. Campbell Brace . . . . . . . . . 228

84. Thomas Knee-Brace . . . . . . . . 230

85-86. Transverse Section of the Forefoot . . . . 25I

87. Callus in Morton's Toe . . . . . . 256 


\section{ILLUSTRATIONS}

FIG.

88. Hallux Valgus . . . . . . . . . . 258

89. Operation for Hallux Valgus . . . . . . 260

9o. Conservative Treatment of Ingrowing Toe-Nail 273 9I-92. The French and Cuban Heels . . . . . . 280

93. A Faulty Shoe-Toe . . . . . . . . . 28I 94-95. The Toe of the Shoe . . . . . . . . 282

96. An Excellent Shoe . . . . . . . . . 282

97-98. Rocker-Sole and Flat-Sole Shoe . . . . . 284 99-100. Alterations in Length of Foot During Rest and Weight-Bearing . • . . . . . . 285 Ior. Alterations in Length of Foot During TipToeing . . . . . . . . . . . 286 


\section{DISEASES AND DEFORMITIES OF THE FOOT}

\section{CHAPTER I \\ ANATOMY}

\section{THE BONES}

THE bones entering into the formation of the foot are: the astragalus, the os calcis, the scaphoid, the cuboid, the internal, middle and external cuneiforms, the five metatarsals, fourteen phalanges and two sesamoid bones under the head of the first metatarsal. Other sesamoid bones are inconstant: the interphalangeal one of the great toe is found in about 50 per cent. of cases according to Pfitzner, quoted by Dwight, and the peroneum, the sesamoid in the tendon of the peroneus longus, occurs in about Io per cent. (Dwight). Variations in the conformation of the bones and in their numbers, changes which are not pathological but which may have important bearings on diagnosis and treatment, have been studied and the results presented in book form by the late Professor Dwight of Harvard University.

The Astragalus is the only bone of the foot entering into the ankle joint. It articulates with both bones of 
the leg. Its upper articular surface is convex anteroposteriorly. The axis of the chief movement in which this surface enters, that is to say, the axis of the most pronounced movement in the ankle joint, is close to the calcaneo-astragaloid joint. It passes considerably below the internal malleolus, but not more than an eighth of an inch below the external malleolus. The tip of the external malleolus is a good guide to this axis. How-

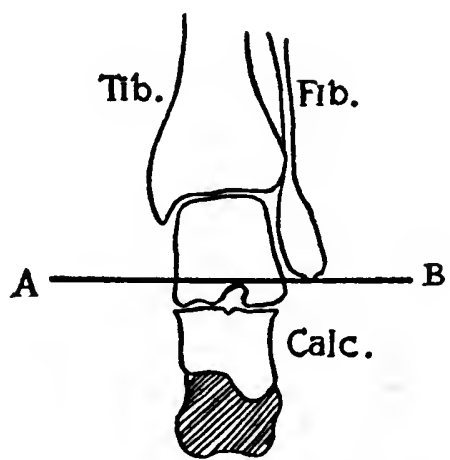

Fig. 1. Axis of Ankle-Joint Movement

The transverse axis of movement at the ankle-joint is represented by the line A-B. It is at the tip of the external malleolus and not through the joint surfaces of the tibio-tarsal joint.

ever, this axis is not stationary; its outer end moves forward during plantar flexion and backward during dorsal flexion. Thus the outer border of the superior articular surface of the astragalus has two movements: the one most marked of rotation about a transverse axis and another, forward and inward or backward and inward, about a vertical axis situated internal to the internal malleolar-astragaloid articulation. The cause of these two 
movements is seen in the shape of the trochlear surface: while the inner edge is almost completely in an anteroposterior plane, the outer edge is so curved that it lies in a plane extending downward and inward. The widest part of the trochlear surface is usually somewhat anterior to the center, the narrowest being at the posterior extremity.

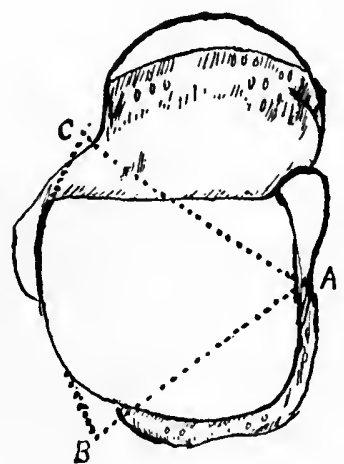

Fig. 2. Trochlear Surface of Astragalus

Left astragalus, from above, demonstrating the arc, B-C, of a secondary movement at the ankle-joint about a vertical axis, $A$.

The articular surface on the internal surface of the astragalus and that on the internal malleolus nearly correspond in size, showing that there is slight movement there other than that of rotation on a transverse axis. The articular surface on the external surface of the astragalus and that on the external malleolus, on the other hand, do not correspond in size, the former being extended much further antero-posteriorly and thus permitting the secondary movement about the vertical axis above described. 
There is no lateral movement, no movement from side to side, at this joint except when the narrowest, the posterior, portion of the trochlear is presented in the mortice formed by the tibia and the fibula. When the widest part of the trochlear is brought directly between the malleoli they are slightly separated and the inferior tibio-fibular ligament is stretched.

The head and neck of the astragalus incline inward toward the center of the foot; but it may be deformed and incline outward toward the other foot, as in congenital club-foot. The head forms the ball of a balland-socket joint, the socket being formed by the scaphoid, by the sustentaculum tali of the os calcis, and by the calcaneo-scaphoid ligament.

Below, the astragalus articulates with the os calcis through both the body and neck, the two joints being separated by the interosseous ligament. The synovial membrane of the posterior joint is separate from that of any other, while that of the anterior communicates with the synovial membrane lining the astragalo-scaphoid joint.

The movements permitted between the astragalus and the os calcis are inversion and eversion on a longitudinal axis and slight rotation on a vertical axis. The movement on an antero-posterior axis, producing inversion and eversion, turns the sole of the foot inward and upward or outward and upward. The former movement, inversion, is the more pronounced. The axis of this movement, to be exact, is oblique; extending from the 


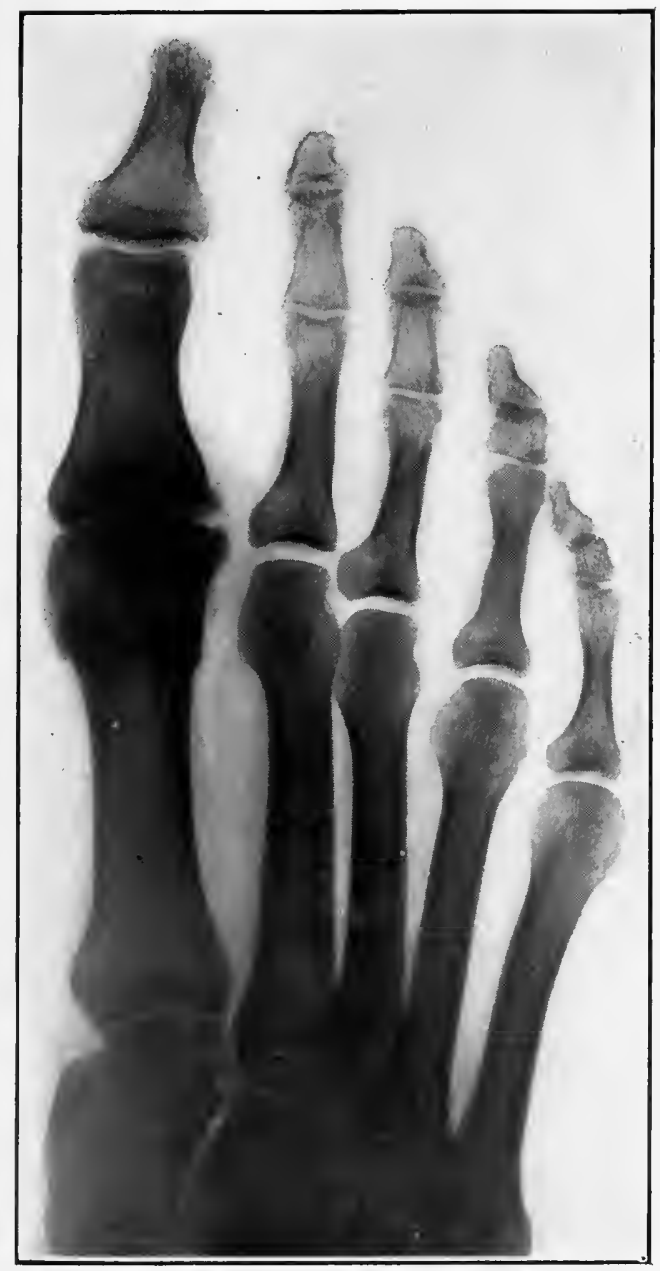

Plate I.-Radiograpi of a Practically Nordal, Forefoot

The slight deviation in the direction of the distal phalanges of the first, fourth and fifth toes from the long axes of their metatarsals shows that the foot-wear has not always been the best fitting. A properly fitting shoe should preserve. the straight alignment of the bones of the toes and also the fan-like expansion of the outer metatarsals. 

upper portion of the head of the astragalus downward, backward, and outward. The rotation through the vertical axis turns the heel inward or outward and the fore-foot in an opposite direction on an horizontal plane.

The Scaphoid assists in forming the socket for the head of the astragalus. Anteriorly it articulates with the three cuneiform bones and, occasionally, externally with the cuboid. On its internal surface is a tuberosity to which is attached a part of the tibialis posticus muscle. This tuberosity is an important landmark in studying deformities.

The Os Calcis, or calcaneum, is the largest bone in the foot. Above, it articulates with the astragalus; anteriorly, with the cuboid. Movement at the calcaneocuboid joint is almost entirely in a downward and inward and in an upward and outward direction. This joint has a synovial membrane separate from any other joint. On the inferior surface of the os calcis are two tubercles: an inner and an outer one. The former is the larger. When the bone is resting on a flat surface its long axis is directed forward, downward, and outward. A tubercle on the external surface, the peroneal tubercle, is situated slightly below and in front of the center of this surface and separates two grooves, through the lower of which runs the tendon of the peroneus longus muscle and through the upper the tendon of the peroneus brevis.

The Cuboid articulates with the os calcis posteriorly; with the two outer metatarsals in front; and, in- 
ternally, with the external cuneiform, and sometimes with the scaphoid. Externally it presents a tuberosity which is close to a groove on this bone for the tendon of the peroneus longus.

The Cuneiform Bones. - The three cuneiform bones articulate in front with the three inner metatarsals.

The Metatarsal Bones.-Of the five metatarsal bones, the first is the strongest and the shortest, while the second is the longest.

The Sesamoid Bones.-Under the metatarsalphalangeal joint of the great toe are the two sesamoid bones. They are in the tendon of the flexor brevis hallucis and are united by a transverse ligament. These bones are not accidental formations due to pressure (Bell), but have been found in the still-born baby (Cross). They are essential to the perfect physiology of the foot.

The Phalanges.-There are two phalanges for the great toe and three for each of the others.

Arches.-In most descriptions of the foot, the bones are divided into two rows and described as forming arches. Each foot is spoken of as forming three arches: an inner and an outer longitudinal arch and an anterior, or transverse, arch. None of these arches is a perfect arch and therefore can have no keystone. In fact, nothing is gained by trying to adopt the terms of an engineer of inanimate structures.

Skeleton of the Foot.-If the articulated bones of a foot are placed upon the table, it will be seen that the 
foot rests upon the os calcis, through its internal and external tuberosities, and upon the heads of the five metatarsals. The cuboid on the outer side is much nearer the table-top than is the scaphoid or the internal cuneiform on the inner side. Neither the scaphoid nor any of the cuneiforms nor the cuboid reach the plane on which rests the os calcis and the heads of the metatarsals. The lower edge of the tubercle of the scaphoid is slightly above a line connecting the posterior edge of the internal malleolus and the inferior tubercle on the head of the first metatarsal. Attention has been drawn to this relation by Feiss.

Just back of the scaphoid is the head of the astragalus, with which it articulates, and below the head is the sustentaculum tali. On the outer side of the os calcis is seen the peroneal tubercle and on the outer side of the cuboid may be seen the groove for the tendon of the peroneus longus. The os calcis projects backward into what is called the tuberosity. It is this tuberosity which forms the projection of the heel, and therefore when the longitudinal axis of the os calcis is altered, as it is in a lowering of the dome of the foot, then the prominence of the heel is altered. Negroes are so frequently afflicted with the deformity of flat-foot, in which the axis of the os calcis is changed from forward, downward, and outward to a direction of forward, horizontal, and inward, and in consequence have such prominent heels that it has been mistaken for a characteristic of the race, instead of a characteristic of an acquired deformity. 
It will be noted that the medio-tarsal joint is not transverse to the long axis of the foot: it lies in a plane which is oblique from within, outward and backward. There are only two bones in the foot back of this joint: the os calcis, articulating with the cuboid, and the astragalus, articulating with the scaphoid. In front of the mediotarsal joint, the cuboid and the scaphoid are not side by side; the three cuneiforms articulate with the scaphoid anteriorly and the cuboid articulates with the external cuneiform.

The five metatarsals, lying side by side, form a dome, convexity upward, much like that which is formed by the metacarpals when the hand is cupped. The two outer metatarsals articulate with the cuboid and the three inner ones with the cuneiforms. There is less movement between the middle metatarsal and the ones on either side of it, than there is between the other metatarsal bones.

It should be noticed that the joints between the heads of the metatarsals and the first phalanges are not in the same plane. The first two joints, those of the great toe and of the second toe, have their transverse axes in alignment and the axes of the outer three form an almost straight line, but the axes of the outer three joints is directed obliquely outward and backward from the axis of movement of the two inner joints. The latter axis is nearly at a right angle to the longitudinal axis of the foot.

The first metatarsal bone is conspicuously larger than any of the other metatarsals. In fact, there is reason for 
considering it the first phalanx, but all we need to notice here is that it is a very strong, stout bone.

The sesamoid bones under its head prevent pressure on the tendon of the flexor longus pollicis while the foot is weight-bearing, so that that tendon is always free to move. These bones also permit the head of the metatarsal to be moved under the same conditions. Upon assuming the position of tip-toe, abduction takes place at this joint, the large toe being moved away from the others.

\section{THE LIGAMENTS}

The ligaments are very numerous between the bones of the foot.

Plantar Fascia.-Among these might be included the plantar fascia, as it has a ligamentous action among its other functions. No one can see this fascia without being impressed with its remarkable strength. It stretches from the tuberosities of the os calcis forward to the heads of the metatarsals and the first phalanges. It is made of white fibrous tissue containing many fat cells. To it is attached the plantar skin, which, therefore, does not move upon it as does the palmar skin on its fascia. The upper or deep surface of this fascia sends layers to the bones to form compartments for the muscles, nerves, and vessels.

Plantar Ligaments.-On the plantar surface, there are two true ligaments of large size: the long and the short plantar ligaments. They extend from the os calcis 
forward, the shorter to the part of the cuboid behind the oblique ridge, the longer to this ridge and, by some of its fibers, to the metatarsal bones. Thus this long plantar, the long inferior calcaneo-cuboid ligament, encloses the peroneal groove and forms a canal for the tendon of the peroneus longus.

On the dorsum of the foot there are the dorsal ligaments of each joint, but none of these demands our special attention.

External Lateral Ligament.-On the outer side of the foot the lateral ligament of the ankle joint is strong and important. It may be divided into three slips: the one most anterior, extends from the anterior border of the malleolus forward and inward to the astragalus; the middle slip extends from the outer surface of the malleolus, close to its apex, to the tubercle on the outer surface of the os calcis, - this part of the ligament is crossed by the tendon of the peroneus brevis; the posterior slip arising from the posterior border of the malleolus and attached at its other end to the astragalus close to the articular facet for the fibula.

Internal Lateral Ligament.-On the inner side of the foot are two ligaments of the greatest consequence in many deformities of the foot. They are the internal lateral ligament of the ankle and the inferior calcaneoscaphoid ligament. The internal lateral ligament of the ankle, the deltoid ligament, is attached to the internal malleolus above, while below its attachments are: behind to the astragalus, in the middle to the astragalus and to 
the sustentaculum tali, and in front to the calcaneoscaphoid ligament and to the scaphoid bone. It is probably this ligament which offers greater resistance to the correction of congenital club-foot than any other structure exclusive of bone.

Calcaneo-Scaphoid Ligament. - The calcaneoscaphoid ligament is an exceedingly strong, thick ligament extending from the sustentaculum tali to the scaphoid. As in some positions it supports the head of the astragalus, it has been called the suspensory ligament; but it is worthy of emphasis that this ligament must be less of a factor in supporting the astragalar head when the scaphoid is rotated downward, - as when the foot is on the ground and the toes are turned outward, - than when the foot is adducted, for the ligament is then relaxed.

Posterior Ligament.-The posterior ligament of the ankle joint, the posterior tibio-astragaloid ligament, may be an important factor in preventing full dorsal flexion at this joint. It lies in front of the narrowest part of the tendo Achillis, opposite the thickest part of the internal malleolus, and is easily divided through the same puncture made by the tenotome for division of the tendo Achillis.

\section{MUSCLES AND TENDONS}

It is well to remember in studying muscles that though the name of the muscle will often indicate one action of which it is capable it is by no means its only action. Most 
of the muscles of the foot cross several joints and each one of these joints is affected by contractions of the muscles crossing it. Therefore a study of the physiology of these muscles is by no means a simple matter. Then, too, a muscle or a group of muscles, acting unopposed will produce one set of movements, while if opposed by the action of another muscle, or muscular group, a different set of motions will be produced. It is an impossibility for any muscle of the foot to produce a movement confined to any one joint, unless its action is counteracted in part by one or more other muscles. Thus flexion or extension of the ankle is not a simple movement: some movement takes place in at least the sub-astragaloid joint. Under normal conditions almost every joint in the foot is affected by the movement in any one joint. It is this close association of joint movements which makes analysis of the movements difficult. For instance, rotation on a vertical axis takes place both in the subastragaloid and in the medio-tarsal joints, and so does rotation on an antero-posterior axis.

However, it simplifies our study, if, with this in mind, we consider the chief axes of the movements of the foot to be three: one transverse, for flexion and extension through the ankle joint; one antero-posterior, passing through the sub-astragaloid joint, for inversion and eversion of the sole; and one vertical, through the mediotarsal joint, for abduction and adduction of the forefoot.

Calf Muscles.-The calf muscles consist of the 
gastrocnemius, the soleus, and the plantarus. The last is a small muscle, seldom inserted into the plantar fascia, but usually into the os calcis with the tendo Achillis. The gastrocnemius and the soleus have very large bellies which form the greater part of the calf. If the other muscles of the back of the leg are undeveloped, the lower

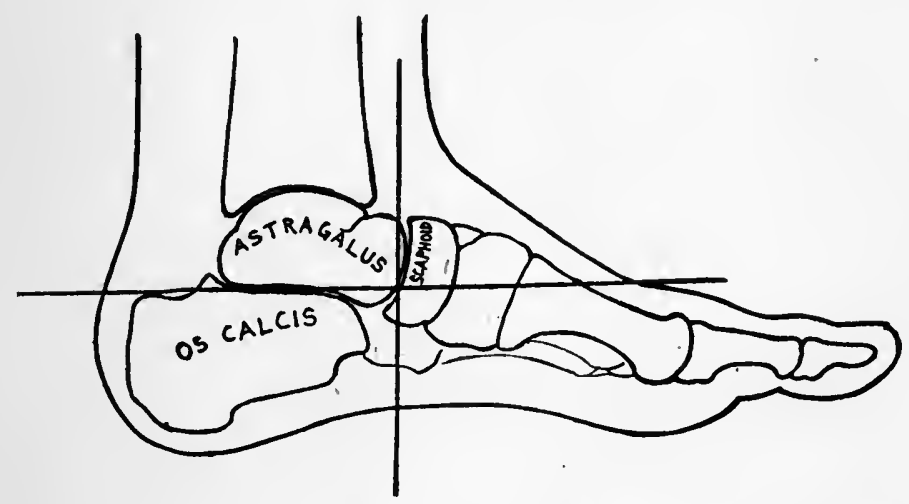

Fig. 3. The Three Principal Axes of Movements of the Foot

They are transverse, longitudinal and vertical. The transverse is shown in cross-section on the astragalus. It should be placed nearer the sub-astragaloid joint. It is here that the movements of the ankle, dorsal and plantar flexion take place. The longitudinal is parallel with the long axis of the foot, through the sub-astragaloid joint. Motion here is in the direction of inversion and eversion of the sole. The vertical is through the medio-tarsal joint, with motion producing abduction and adduction of the fore-foot.

third of the leg appears very small and the calf seems situated higher up than normally. The leg of the dancing girl, with the muscles all well developed, is much more graceful than the leg of the laborer, whose gastrocnemius and soleus are out of all proportion to the development of the other muscles. 
The gastrocnemius is attached above to the femur and below to the os calcis through the tendo Achillis, common to it and the soleus. Thus the influence of this muscle is felt in three joints: the knee, the ankle, and the sub-astragaloid. The muscle is stretched to its greatest extent when the knee is fully extended, the ankle dorsal flexed, and the foot inverted. If the knee is flexed, the dorsal flexion and the inversion can be increased. Therefore in testing the length of this muscle it is essential that the knee be in extension. The soleus is attached to both the leg bones and to the tendo Achillis.

Tendo Achillis. - The tendo Achillis is not inserted directly into the posterior part of the os calcis, but its fibers should be considered as passing around the tuberosity into the plantar surface, although these fibers are more or less ossified. Thus the force exerted through this tension is more of a lifting and a thrusting forward than of a direct pulling upward. The insertion of the tendon is not exactly in coincidence with the long axis of the os calcis, but is slightly to the inside, so that it has a tendency to turn the ankle outward and to invert the sole of the foot when drawn on by contraction of the calf muscles. Thus it will be seen that the muscular action serves to emphasize that outward thrust of the ankle, when the weight-bearing foot is raised on tip-toe, which must take place on account of the shape of the trochlear surface of the astragalus and to which attention has been called in discussing that bone.

Between the tendo Achillis and the posterior part of 
the tuberosity is a bursa which is subject to injury and disease.

The narrowest part of the tendo Achillis is one and one-half inches above the heel, at a point about opposite the thickest part of the internal malleolus, and here it is not closely associated with important structures and is therefore the site of selection for subcutaneous tenotomy.

Peronei.-The two peroneal muscles have attachment to the fibula, their tendons passing downward behind and close to the external malleolus. The tendon of the brevis lies in front of the tendon of the longus in this location. On the outer surface of the calcaneum the brevis lies above the longus and here they are separated by the peroneal tubercle. Here each tendon has its separate synovial sheath. The brevis is inserted into the fifth metatarsal. The longus, at the tubercle of the cuboid, enters the groove on that bone and runs diagonally forward and inward to be inserted into the outer surface of the base of the first metatarsal and the corresponding surface of the internal cuneiform close to the cuneiform-metatarsal joint.

The action of these two muscles is to plantar-flex the ankle, to evert the foot on the longitudinal axis passing through the sub-astragaloid joint and to abduct the forefoot on the vertical axis passing through the mediotarsal joint. The brevis has a very weak abducting action, but greatly strengthens the calcaneo-cuboid joint and slightly flexes it. The longus draws the inner border of the foot toward the cuboid and heightens and greatly 
strengthens the dome of the foot. Furthermore, in this action it rotates the inner cuneiform and the first metatarsal inward, and thus crowds upward and outward the scaphoid, which in its turn brings a pressure to bear against the head of the astragalus. It should be noted well that the peroneus longus can act much better in holding down the forefoot and in strengthening the dome if the foot is in adduction rather than abduction.

Structures at the Inner Ankle.-Behind the inner malleolus pass the tendons of three muscles together with the posterior tibial vessels and nerve. Just back of the thickest part of the malleolus these structures lie in the following order: close to the bone, the tendon of the tibialis posticus; next, that of the flexor longus digitorum; then a vein, the artery, and the other vein; then the posterior tibial nerve; and finally the tendon of the flexor longus pollicis. The first letter in each word in the following sentence corresponds with the first letter in the name of each structure in its proper order, as is known to many students: Timothy doth vex all very nervous people.

Flexor Longus Pollicis.-This muscle arises from the fibula. Passing downward, its tendon enters the outer groove on the back part of the tibia; then passes between two tubercles on the posterior surface of the astragalus and turning inward and forward passes under the sustentaculum tali; next crosses the tendon of the flexor longus digitorum, passing above it; and proceeds forward between the sesamoid bones under the head of 
the first metatarsal to be attached to the base of the second phalanx.

Action of this muscle produces at the ankle joint plantar flexion; at the subastragaloid, slight inversion; at the medio-tarsal, slight adduction; and flexion at the astragalo-scaphoid, the scapho-cuneiform, the cuneiform-metatarsal and the metatarsal-phalangeal joints. If the great toe is held rigid against the ground by the action of the short plantar muscles attached to it and by the weight of the body and the heel be raised, contraction of the flexor longus pollicis will throw the ankle outward and forward and, by flexion of the abovementioned joints, will raise the vault and shorten the distance between the heel and the toe. In this position of the weight-bearing foot, the great toe is drawn away from the other toes and rotated upon its longitudinal axis. Under ordinary conditions flexion of the first phalanx is prevented by the action of the flexor brevis, the abductor, and the adductor pollicis. If the extreme tip-toe position is assumed and the last phalanx only is on the ground, the position habitually used in dancing the ballet, the metatarsal-phalangeal joint rides on the tendon of the flexor longus pollicis and the sesamoids are lifted from the supporting surface.

Contraction of the flexor longus pollicis, therefore, does not flex the great toe unless it is desired to grasp something, and "grasping is not a function of the great toe when it is exerting its greatest strength." When the foot is at rest the metatarsal-phalangeal and the inter- 
phalangeal joints may easily be flexed, but the foot in action, or ready for action as in assuming a position for springing, has the first phalanx held securely against the metatarsal and the inter-phalangeal joint equally immobilized. In this respect the great toe is in strong contrast to the other toes; under contraction of their common flexor they flex at their first inter-phalangeal joints and extend at the distal joints, the flexor surface of the last phalanx being held against the ground, but the proximal inter-phalangeal joint being raised, flexed. These toes may, however, for prehensile purposes be made to flex at all their points, but that is not their strongest nor their usual action. It will be seen from the foregoing that, in order that the flexor longus pollicis may act properly, it is necessary that the flexor brevis, the abductor, and the adductor pollicis act normally. A patient with paralysis of these small plantar muscles but with the other muscles of the foot and leg intact, will complain that in walking it is difficult to raise the heel, that he must bring the foot forward in its straight, rightangled position, and that when he attempts to rise on tiptoe his foot feels very weak. He may not be able to do so at all. Upon examination it will be seen that when he rises on tip-toe, the great toe bends up and flexes at the inter-phalangeal joint. Holding this joint down by finger-pressure enables the patient to execute the tip-toe movement more easily. It is likewise essential for the normal functionating of this muscle, that the metatarsalphalangeal joint of the great toe be normal. Deformity, 
such as hallux valgus, or clothing of the foot which prevents normal movements, especially abduction, must interfere seriously with its actions.

A word further is needed as to the thrusting out of the ankle during contraction of this muscle when the forefoot is held immobile, as by the weight of the body in

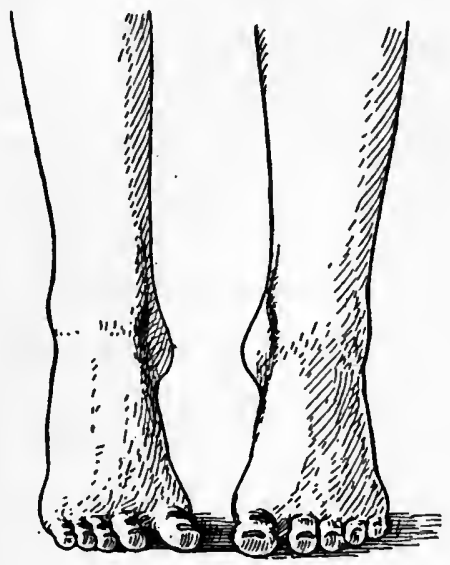

Fig. 4. Plantar Flexion in Walking

Plantar flexion of the weight-bearing foot, in standing and walking, thrusts the ankle upward, outward and forward as the forefoot can not be moved downward, inward and backward-the direction it takes when the free-foot is plantar flexed.

walking: its power, together with that of the flexor longus digitorum and of the tibialis posticus, would tend to produce this movement, as they are all on the inner side of the joint; but the greatest factor in its production is the formation of the tibio-astragaloid joint. With the forefoot resting on the ground, the movement producing the adduction seen in extreme flexion of the 
free foot, produces the outward movement of the ankle. This turning outward of the ankle when the heel is raised can not take place unless the feet are parallel or adducted, and therefore the correct understanding of this movement and its mechanism is of the greatest importance in determining the proper positon of the feet in walking and standing.

A common idea of the function of the flexor longus pollicis is expressed in Morris's Anatomy: "A strong flexor of the last phalanx of the great toe and of great importance in walking, as it presses the great toe firmly against the ground." As it is scarcely ever used as a flexor of the great toe, flexion should not be considered as one of its prime functions and though the muscle is of great importance in walking we do not believe it is used to press the great toe against the ground except, possibly, just before the heel is raised.

The Flexor Longus Digitorum arises from the tibia and is inserted into the last phalanx of each of the four outer toes. As the tendon passes around the ankle, it is separated from the tendon of the flexor longus pollicis by the nerve and the artery with its venæ comities, and lies close to and on the outer side of the tendon of tibialis posticus. That is, the tendon of the tibialis posticus lies between the tendon of the flexor longus digitorum and the internal malleolus. From this point it runs forward, outward, and downward between the abductor pollicis and the flexor brevis below and the tendon of the flexor longus pollicis above. The action of 
this muscle is to assist in moving the ankle upward, forward, and outward; to flex and to adduct the forefoot and to flex the metatarsal-phalangeal and the inter-phalangeal joints of the four outer toes. This action, however, is modified by the accessorius, the flexor brevis, and the lumbricales.

Accessorius.-This small muscle from its origin at the tubercles of the os calcis, draws directly backward the tendon of the long flexor to which it is attached at its curved position in the plantar, thus altering the direction of its force from one of marked adduction and internal rotation to one more nearly in line with the long diameter of the foot.

Flexor Brevis Digitorum.-By its insertion into the middle phalanges of the four outer toes, this muscle flexes the first inter-phalangeal joints and holds the second inter-phalangeal joints in extension when the foot is weight-bearing.

Lumbricales.- They arise from the tendons of the flexor longus digitorum and are inserted into the tendons of the extensor longus digitorum upon the dorsum of the first phalanges of the four outer toes. Their action is to extend the phalanges and thus to assist in holding the plantar surfaces of the distal phalanges to the ground. They will also, by adducting the outer three toes and by abducting the second toe, tend to preserve the transverse arch. They also assist to a slight extent in flexing the metatarsal-phalangeal joints of these toes. Thus it will be seen that the usual action of the flexor longus digi- 
torum is not to flex the toes as though about to grasp some object, - they are not dug into the ground as represented by some sculptors, - but that its action is to flex the four smaller toes at their first inter-phalangeal joints while the distal phalanges are held flat against the ground.

Tibialis Posticus.-This is stronger than either of the other muscles passing behind the inner malleolus. It arises from both the tibia and the fibula, its tendon passing beneath that of the flexor longus digitorum and reaching the innermost groove on the back of the internal malleolus. It then passes forward to be inserted into the tuberosity of the scaphoid. Expansions of this tendon are inserted into the sustentaculum tali; the three cuneiform bones; the cuboid, and the second, third, and fourth metatarsals. In passing from the groove on the tibia the tendon passes beneath the inferior calcaneo-scaphoid ligament from which it is separated only by the synovial sheath of the tendon. It is this ligamentous support to the astragalus which, according to some surgeons (Hancock), is the influence whereby the tibialis posticus helps to preserve the normal dome of the foot. Undoubtedly, when one is standing, especially in the vicious position of abduction of the forefoot, this tendon does act as a more or less passive support; but in action, the adduction and inversion which it produces, together with flexion of the medio-tarsal joint, so opposes any descent of the head of the astragalus that no ligamentous action is present or necessary. By the wide extent of its insertion into the under surfaces of the other bones, and 
especially into the second, third, and fourth metatarsals, its influence in preserving the transverse arch is also of importance.

There are four muscles passing from the front of the leg across the ankle to the foot. They are the tibialis anticus, the extensor proprius pollicis, the extensor longus digitorum, and the peroneus tertius.

Tibialis Anticus.-This muscle arises from the upper two-thirds of the outer surface of the tibia. When well developed it may project in advance of the crest of that bone, but it never overlaps it. When it is atrophied a sulcus may be felt between the crest and the muscle's belly. Its tendon is inserted into the internal cuneiform on the lower part of its internal surface and into the adjacent part of the first metatarsal.

The action of the tibialis anticus is usually described as being that of a dorsal flexor of the ankle and at the same time an elevator of the inner border of the foot. The chief cause of flat-foot has been ascribed to overwork and partial paralysis of this muscle (L. A. Sayre). The condition of this muscle is undoubtedly an etiological factor in some cases of flat-foot, but it would seem that the weakness in uncomplicated conditions is due to improper use of the muscle rather than primarily to the muscle itself, and the improper use is rather a lack of work than over-work. Its action at the ankle is to lessen the angle between the foot and the leg, but whether this action should be expressed as flexing the foot or as flexing the leg depends on which is the fixed and which is 
the movable attachment. This question will be referred to again in discussing the succeeding muscles. Acting from above this muscle has an adducting and an inward rotating force on the internal cuneiform and the first metatarsal and tends to cause an inversion of the sole. The changed position of the internal cuneiform and of the first metatarsal places them in the most advantageous position for supporting the weight of the body.

The Extensor Proprius Pollicis arises from the fibula and is inserted into the first and second phalanges of the great toe.

The Extensor Longus Digitorum arises from the fibula and partly from the tibia and is inserted into the three phalanges of the four outer toes and into the ligament over the metatarsal-phalangeal joints.

The Peroneus Tertius arises from the fibula and is inserted into the fifth metatarsal.

These muscles on the front of the leg have generally been considered as acting from their upper attachments only. The names of two of them imply that they are first of all useful as extensors of the digits. One of their functions is undoubtedly to hold the forefoot and the toes in extension and thus to give a firm support against which the posterior muscles may act, but of greater importance is their function of flexing the leg on the foot. It can not truthfully be said that barefooted people need these great extensors of the toes - and the same holds true for the long flexors - and that modern foot- 
wear has done away with their usefulness. Those who have developed the prehensile power of the toes have done so mostly through the development of the plantar muscles.

Of the plantar muscles, the accessorius and the lumbricales have been described.

The Abductor Pollicis is a strong muscle inserted into the inner part of the lower surface of the base of the first phalanx of the great toe and into the internal sesamoid bone. Its action is to flex the first phalanx and to abduct the great toe (toward the middle plane of the body). This action will draw the metatarsal inward toward the other foot into a position which supports the internal cuneiform and the scaphoid to the best advantage. Thus it produces flexion and abduction of the astragaloscaphoid and of the cuneiform-metatarsal joints, but its action at the metatarsal-phalangeal joint is, normally, to support that joint by compressing the articular surfaces s.rongly together.

The Flexor Brevis Digitorum is a comparatively weak muscle. It is inserted into the middle phalanx of each of the four outer toes and by flexing the first interphalangeal joint helps to hold the plantar surface of the last phalanx to the ground during action of the long flexor.

The Abductor Minimi Digiti is inserted into the base of the fifth metatarsal and into the first phalanx of the little toe. Its action is probably to assist in altering 
the weight-bearing surface of the ball of the foot to accommodate it to uneven surfaces and to the changing positions of the foot in walking and running.

The Flexor Brevis Pollicis is inserted into the first phalanx of the great toe. It is not only a flexor of the metatarsal-phalangeal joint, but a slight adductor as well, as its origin from the cuboid is to the outer side of the middle line of the foot. Its tendons on either side of the head of the metatarsal are attached to the sesamoid bones and not the least important of its actions is to control these bones.

The Adductor Pollicis is also attached to the first phalanx. The great toe, then, has three strong muscles to the first phalanx. Their action is more especially to support the joint strongly when the long flexor is contracted. The abductor is larger than the adductor and abduction is the more important of the two movements in altering the weight-bearing surface, in preserving the transverse arch, and in supporting the clome.

The Transversus Pedis is a small muscle which assists the abductor minimi digiti in adjusting the ball of the foot to the varying pressures.

The Interossei consist of three plantar and four dorsal muscles. Their common action is to flex the first phalanges and to extend the other two of the four outer toes. This they do by virtue of their insertions into the first phalanges and into the tendons of the extensor longus digitorum. It is mostly due to them, assisted by the lumbricales, that the toes are not flexed in walking. 
The Extensor Brevis Digitorum is the only muscle on the dorsum of the foot. Of its four tendons, the innermost is attached to the first phalanx of the great toe and the outer three to the tendons of the extensor longus digitorum going to the second, third, and fourth toes. The obliquity of the direction of these three outer tendons assists in correcting the direction of pull by the tendons of the extensor longus digitorum.

\section{NERVES}

The nerve supply of all the muscles of the foot comes from two main trunks: the internal and the external popliteals. The internal popliteal, as such, or after it becomes the posterior tibial or divides into the internal and external plantars, supplies all the muscles on the back of the leg and all the plantar muscles. The external popliteal, after dividing into the anterior tibial and the musculo-cutaneous, supplies the muscles on the anterior and outer surfaces of the leg and on the dorsum of the foot.

The cutaneous nerves of the foot are derived from the long saphenous, a branch of the anterior crural, which descends through Hunter's canal and becomes subcutaneous in the upper part of the outer side of the leg; from the short saphenous, which is formed in the middle of the calf by branches from the popliteals, the communicans tibialis, and the communicans fibularis; from the cutaneous branch of the musculo-cutaneous; and by cutaneous branches from the anterior and posterior tibials. The 
outer border of the foot is supplied by the short saphenous; the inner border by the long saphenous; both ankles and the dorsum by the musculo-cutaneous, which also supplies the dorsum of the adjacent sides of the second, third, and fourth toes. The adjacent sides of the first and second toes are supplied by the anterior tibial. The sole is supplied by the internal plantar, as is also the inner border of the first toe and the adjacent borders of the second, third, and fourth toes and the structures about the nails. The external plantar supplies the adjacent sides of the fourth and fifth and the outer side of the fifth toe. 


\section{CHAPTER II}

\section{PHYSIOLOGY}

Distribution of Weight.-A study of the structure of the bones, and especially of their striæ, as indicating the direction in which nature is prepared to receive the stress of weight and power, shows that the greatest strength of the astragalus is in the body extending to the os calcis, but that the head and neck are exceptionally strongly made. A comparison of the internal architecture of the head of the astragalus and the scaphoid with that of the bones about the calcaneo-cuboid joint demonstrates that the former are fitted to receive the greater stress.

When the tibiæ are in the transverse plane of the body containing the center of gravity, the amount of weight transmitted through the posterior tubercles of the os calcis and the amount transmitted through the astragaloscaphoid articulation, will depend on the flexion of the foot. The greater the dorsal flexion, the greater will be the weight transmitted through the os calcis; and the greater the plantar flexion, the greater will be the weight transmitted through the scaphoid. However, it must be borne in mind that the position of the transverse plane containing the center of gravity will influence the share 
of the weight borne by any particular bone. This may be easily demonstrated by scales: if a stand is erected so as to be contingent to and level with the scale-platform, it will be readily seen that moving the transverse plane ever so slightly changes the proportion of the weight passing through the forefoot and the heel. With the subject so placed that the heels are on the stand and the forefoot rests upon the scale-platform, advancing an arm, nodding the head, contracting the abdominal muscles, raising the chest, will cause an alteration in the reading of the dial. It is hard to conceive of this plane remaining unchanged for any length of time. Though a man weighing I 50 pounds and standing in low-heeled shoes may be said to transmit Ioo pounds through the heels and 50 pounds through the forefoot, and this was the conclusion reached by Sampson after a careful mathematical calculation, it must not be supposed that even while one is standing the relation of these two weights is constant. One reaches forward, leans backward, bends the neck, makes a gesticulation, - and the ratio between the weight borne by the forefoot and that borne by the heel must suffer alteration. In short, no ligament, muscle, or bone is under a constant amount of strain while the body is in the upright position. The sense of balance will cause the plane of the center of gravity to be quickly returned when moved from its normal position; but changes in the amount of strain borne by individual bones, ligaments, and muscles will have occurred, even if momentary, and that is of the greatest importance. 
MOVEMENTS

The arrangement of the bones of the foot and their peculiar shapes have caused not a little controversy as to their physiology. The only movement of the foot which is invariably examined, and usually imperfectly, is the movement at the ankle joint. Were the foot comparable to the rim of a wheel, as Dr. Holmes pictured it, the intrinsic foot movements would be of slight value. But as every joint, with its every movement, is essential to the perfect usefulness of the foot and as knowledge of these structures is not widespread even among physicians, it is not a cause for astonishment that the pseudoscientist has found the field of aching feet an especially profitable one.

Ankle-joint.- The most marked movement at the ankle joint is that of flexion and extension. To avoid confusion they are frequently designated as dorsal and plantar flexion respectively. Dorsal flexion takes place normally to 20 degrees beyond a right-angle ( 18 , Tubby: $15-20$, Whitman). That is, with the foot upon the ground the leg can be flexed on the foot until it forms an angle of 75 or 70 degrees with the sole of the foot. If this dorsal flexion is limited, as when the tibia can be flexed only to a right-angle, an abnormal condition exists and proper functionating of the foot is interrupted. Plantar flexion of the foot is more free, extending to form an angle of about 150 degrees with the leg. As explained in the chapter on anatomy, these are not the 
only movements at the ankle. Owing to the convexity of the outer border of the trochlear of the astragalus, the anterior portion of that bone is turned slightly inward in full plantar flexion and less markedly outward in full dorsal flexion. This may be seen in the outward
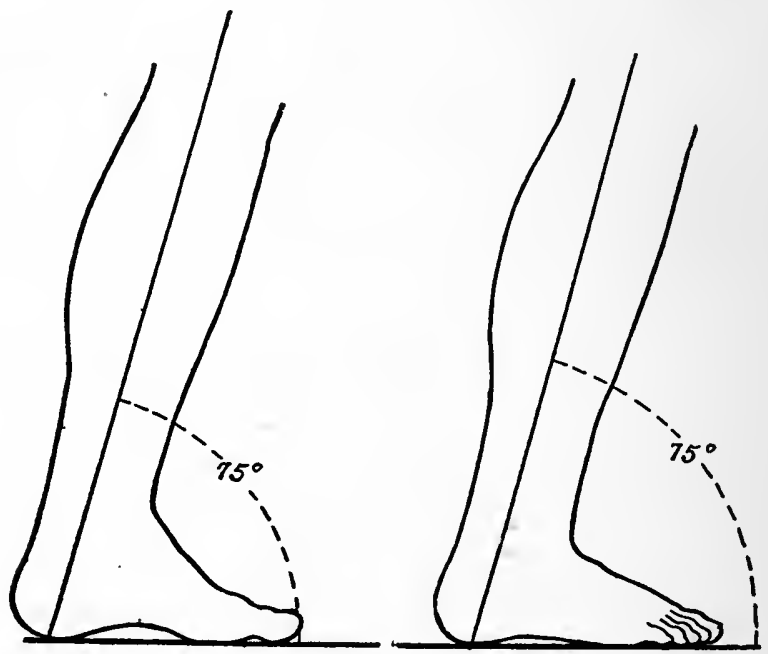

Fig. 5. Tibial Flexion

Dorsal flexion of the weight-bearing foot may be termed tibial flexion. Normally the leg can be flexed on the foot to form an angle of 75 degrees with the ground.

thrust of the ankle when one is rising on tip-toe; the weight of the body prevents the toes being carried inwards, and therefore as plantar flexion progresses the ankle is forced outwards:- a most important fact to consider when studying the physiology of walking. 
At the ankle joint, then, there is normally plantar flexion with adduction and dorsal flexion with slight abduction.

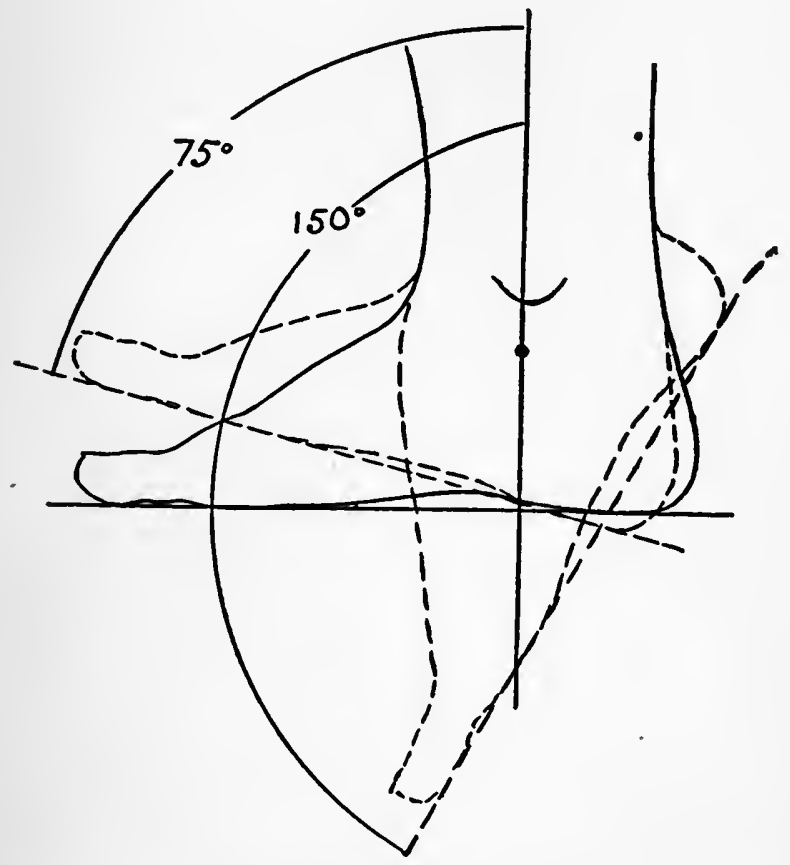

Fig. 6. Movements at the Ankle-Joint

The axis of these movements is situated one-half an inch below the internal malleolus. Dorsal flexion is arrested at an angle of 75 degrees and plantar flexion at an angle of 150 degrees with the line of the leg.

Sub-astragaloid Joint.- The chief movements at the calcaneo-astragaloid, the sub-astragaloid joint, are inversion and eversion, by which the sole is made to 
look inward and outward. With these movements there is some abduction and adduction, some rotation about a vertical axis.

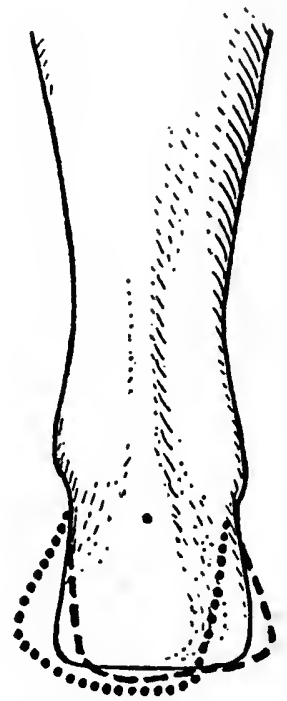

Fig. 7. Movements at the Sub-Astragaloid Joint

Movements at this joint are on an antero-posterior axis, in the direction of inversion and eversion. The dotted line shows range of inversion and the dashed line the range of eversion.

Medio-tarsal.- The medio-tarsal joint comprises two joints: the astragalo-scaphoid and the calcaneo-cuboid. It is usually spoken of as one and is called the medio-tarsal or mid-tarsal joint. The chief movement here is about a vertical axis in the direction of abduction and adduction. With this there is some rotation on an antero-posterior axis, producing inversion and eversion, 
and also some movement on a transverse axis producing flexion and extension.

Anterior to the medio-tarsal joint, the arthrodial joints have only slight movements, but they are essential to the normal use of the foot. The metatarsal-phalangeal joints, especially the first, may, by an abnormality, cause serious loss of function.

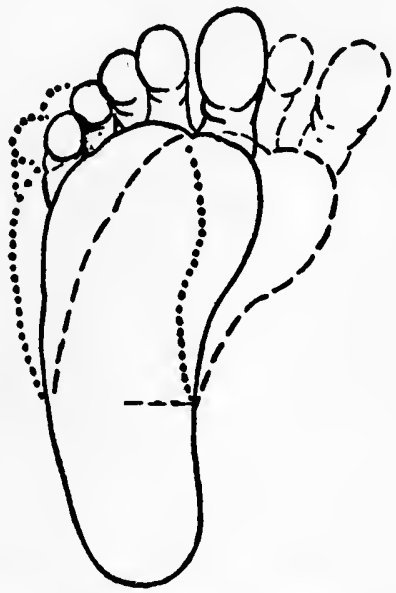

Fig. 8. Movements at the Medio-Tarsal Joint

These movements are chiefly abduction and adduction about a vertical axis. The dotted line shows range of abduction and the dashed line the range of adduction.

There are, then, three principal axes of movement in the foot: one, transverse through the lower part of the astragalus, where most of the extension and flexion takes place; one horizontal, or nearly so, extending anteroposteriorly through the sub-astragaloid joint, where inversion and eversion are the most marked; and the third 
axis, vertical through the medio-tarsal joint, where abduction and adduction of the forefoot are the most marked movements. Active abduction without eversion or active adduction without inversion is very limited, although passively they may be demonstrated.

Great-toe Joint.- The first metatarsal-phalangeal joint is deserving of special notice. It is a condyloid joint, but motion is chiefly in a plane downward and inward, toward the middle line of the body, away from the other toes. At rest the toe is slightly dorsal-flexed and drawn toward the other toes. In the active position it is in a line with the foot, neither flexed nor extended, and is abducted, drawn away from the other toes. In the acting normal foot, therefore, there is a space, a separation, between the great toe and the second toe. This alteration in the position of this toe during rest and during activity has an important bearing upon the causes and cure of flat-foot and upon the designing of foot-wear.

\section{THE FOOT AT REST}

With all the muscles at rest the foot is plantar-flexed, adducted, and rotated slightly inward. This may be seen in the anesthetized patient, in the perfectly relaxed, supine position and in the crossed-leg position. During muscular rest there is ligamentous strain if the foot is not supported by some extraneous means. The plantarflexed position will not cause strain of the plantar ligaments, but of the dorsal ligaments; and the inward rotation, while relaxing the internal ligaments, will strain 
the external. If this strain is allowed to continue for a prolonged period, the ligaments become weakened and more and more strain will be transferred to the muscles. Some changes may take place in the articulations, the most marked being the prominence on the dorsum of the trochlear surface of the astragalus, due to the exaggerated plantar flexion of that bone. This passive strain may result in nothing more serious than a weak-foot; or a peripheral neuritis or even a paralysis may follow. During a protracted illness therefore it is incumbent on the physician to see that measures are taken to prevent this foot-drop and its possibly attending evils. In the weakened condition of all the body structures, including the muscles and ligaments, which accompanies protracted illness, such as typhoid fever, as well as when the muscles of the foot and leg are weakened from a partial or complete paralysis, some means should be taken to retain the foot at about a right-angle with the leg. A prolific cause of continued paralysis following anterior poliomyelitis is this passive stretching of muscles; this subject will be taken up more fully when that disease is discussed.

\section{STANDING}

Were the foot jointless except for the tibio-astragaloid joint, its physiology would be much simpler than it is with its no less than thirty-eight articulations. Many indeed use the foot as though it were one solid mass and more than one surgeon has described it as though its normal movements were limited to flexion and extension at 
the ankle joint. Were this the case the question of the best standing position would be limited to the best geometrical figure to be formed by the two feet; their distance apart, their relation to each other, and the angle the foot should form with the leg. There can be no doubt but that we should select the quadrilateral rather than the trapezoid figure for the feet. That is, we should have the centers of the balls of the feet and the centers of the heels form the corners of a square. If the heels are
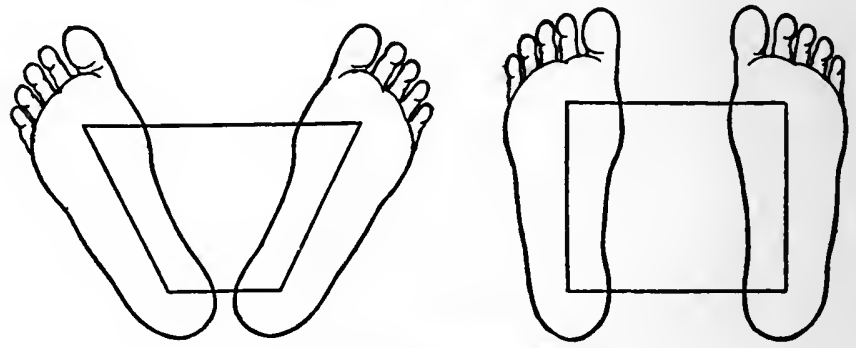

Fig. 9. Standing Positions

Trapezoid and quadrilateral bases of support. The former is decidedly the weaker, not only mechanically but physiologically, on account of the weak position of the bones, ligaments and muscles. (After Ellis.)

placed nearer together and the toes turned outward, a trapezoid figure is formed, which is a decidedly weaker base for support than the square (Ellis).

To bring the transverse plane containing the center of gravity of the body over the strongest part of the bony structures of the foot and to preserve it there with the least muscular exertion, we should place the foot at about a right-angle with the leg. Thus the body of the astragalus would rest mostly on the os calcis. Even with the 
most careful balancing, however, a condition of absolute equilibrium could be retained only by having the flexors and the extensors of the ankle in almost constant action, correcting the positions of the tibia as the gravity-plane was moved backward or forward.

Work, Rest, Fatigue.- It may be well to here discuss work, rest, and fatigue. If the muscles as described above are in constant action, they are usually given credit for continuous work. The action, however, is not, strictly speaking, constant; it is intermittent. Normal growth of a healthy muscle, and in fact of every physiological tissue, demands both work and rest. With the former alone, fatigue must result; and with the latter alone, atrophy. Either condition may be produced to a serious extent, even beyond repair. Fatigue depends upon the relation of work to rest and is very variable. The corresponding muscle in different individuals varies in a wide range not only as to the work it can do without rest and without fatigue, but in the same individual this potentiality must be affected by a great many factors having to do with the functions of other tisues.

In proper standing, no one muscle is in constant action. The amount of rest each muscle enjoys is or should be quite as definite as the amount of work, and in a healthy individual fatigue should not occur within a reasonable time. There are muscles in the body which undergo intermittent contraction for most of the waking hours, and some muscles, the cardiac for instance, work and rest for the lifetime without any prolonged rest. 
The foot can not be considered as a solid mass, as though it were composed of one bone. The modern clothing of the foot hides the more minute movements from view, but those movements are none the less made, and if they are prevented or even limited, the mechanics of the foot are altered and a weaker organ results. Whether or not such weakness becomes a serious disability depends upon the extent of the interference and upon the amount the confined foot is used.

If the foot is resting on the ground in a relaxed condition and is called into action by one's quickly rising to an upright position, certain changes in the positions of the bones take place. The most evident of these is the abduction; - toward the middle line of the body, toward the other foot - of the great toe, the first metatarsal, the internal cuneiform, and the scaphoid. Whereas in rest all the toes are in contact, a space is now evident between the first and second toes. With this movement there also occurs a rotation of the same bones upward and inward, rotation downward on that side toward the middle line of the foot. There will also be an increase in the height of the scaphoid above the ground and an increase in the height of the dome, due mostly to the abduction, rotation, and slight flexion of the joints already mentioned. The transverse arch will be increased, and the four outer toes will be flexed at their first inter-phalangeal joints and extended at their distal joints. The cuboid will be slightly flexed and adducted and there will be some, flexion at the two outer 
scapho-cuneiform articulations. There will be some rotation inwards on a vertical axis at the subastragaloid joint.

These movements will be produced, by the plantar muscles abducting the first phalanx of the great toe and holding it firmly against the metatarsal and the ground; by the long flexor of the great toe acting on all the joints over which it passes; by the tibialis anticus drawing upward and rotating inward the scaphoid and the internal cuneiform; by the tibialis posticus drawing backward, flexing the scaphoid; and also flexing and abducting drawing away from the middle line of the foot - all the bones to which it is attached by the wide expansion of its insertion; by the long flexor of the toes, which, while pressing the distal phalanges to the ground, assists the tibialis posticus in rotating the foot inward at the sub-astragaloid joint; by the plantar muscles flexing the joints in the forefoot and thereby increasing the transverse arch, in which action the peroneus longus plays a very important part. The foot is not more inverted, the forefoot more markedly adducted, and the toes crumpled up in complete flexion because the peronei, the small adductor of the great toe, the long extensors of the toes, the lumbricales and the interossei are acting at the same time. The importance of every one of these muscles is seen where one or more have been paralyzed. 


\section{WALKING}

The question of what may be called the physiological way of walking can be determined by deciding whether the heel or the toe should be presented first to the ground. A few minutes' observation of the pedestrian in any large city will convince one that most people walk on their heels. On the other hand no runner would think of letting his heels touch first. The athletic trainer is continually cautioning his men to "keep up on your toes."

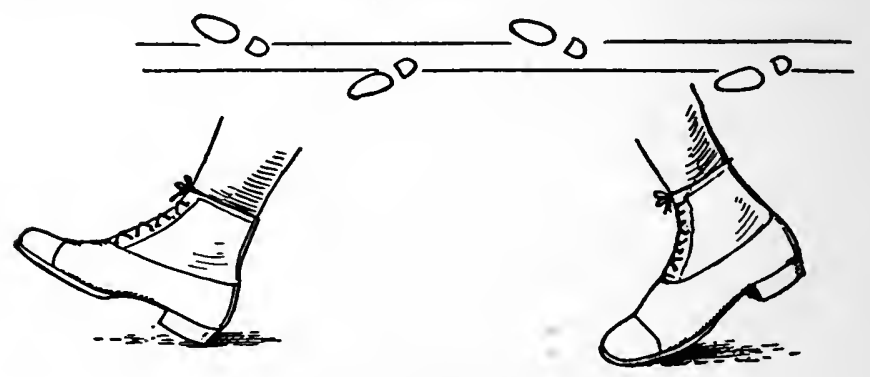

Fig. Io. Heel-Walking

The feet are abducted; toes turned outward. This is conducive to awkwardness, fatigue, weakness, strain and deformity.

Even a heel-walker presents his toes first if he steps down from any height, as from a high curb. No one would think of jumping from a height of a few feet and landing on his heels. Therefore it would seem to be quite evident that less concussion is felt and more spring is obtained by presenting the toes in advance of the heel. That all movements are more gracefully executed with the heels off the ground is known to dancing-masters. Many years ago, teachers of deportment are said to have 
adopted a novel way of training young ladies not to walk on their heels; a rubber ball having a whistle attachment was so placed in the heel that if one did not walk on her toes in crossing the ballroom floor, the whistle sounded at each step.

To the conclusion that for these reasons alone walking should be done on the toes, one might reply, however, that walking is not running, nor jumping, nor dancing. Yet why should the foot, which suffers less fatigue, transmits less shock, and gives more grace when the toes are brought to the ground in advance of the heel, be changed in its action so as to present the heel first when walking? As for the fatigue, the heel-and-toe walking is a far more fatiguing exercise than running, according to the witness of those who have entered these contests, which are rapidly being eliminated from athletic programmes. As for the shock, it may be very slight at each step; but that there is a shock, which is felt in the cord and brain is quite evidenced by the toe-walking temporarily adopted by anyone suffering with a severe headache. It is for the lessening of this concussion, too, that rubber heels are worn. As for the grace, no comparison is necessary. Toe-walking is frowned on by some as being an expression of affectation, but such a reason for ignoring physiological laws is too inane for discussion.

Nevertheless many are convinced that the usual manner of walking is the correct one. They argue, that the heel presentation is by far the most common; that people adopt it without being taught; and that in bringing the 
foot forward it seems to entail less effort to present the heel. Doctor Holmes represented walking by a wheel, the legs being the spokes and the feet representing segments of the rim. Were this a true interpretation of the act, the heel would of course reach the ground before the toes. The only one of the objections which is a true obstacle to the general adoption of toe-heel walking is that heel-toe walking is so very common. It must

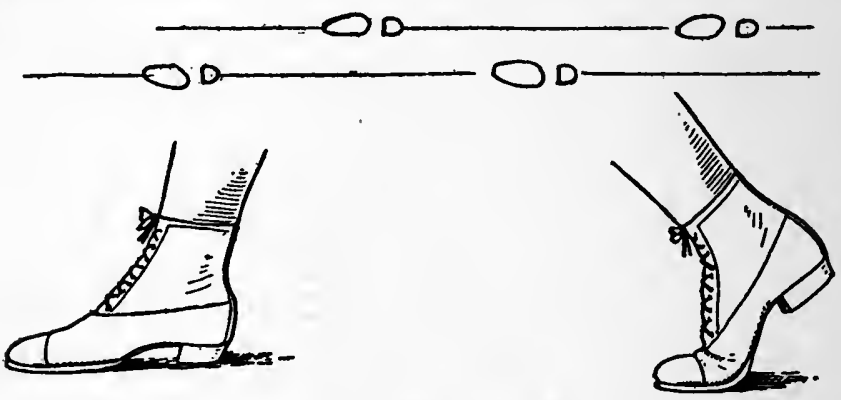

Fig. II. Toe-Walking

The feet are directed in a line parallel to the line of progression. The toe reaches the ground slightly in advance of the heel. Dorsal flexion of the back-foot (flexion of the leg) takes place in a normal manner. This is conducive to grace, rest and strength.

necessarily be a slow reformation to change a custom so generally adopted by all civilized races. The reason that it is so much easier to walk on the heels rather than on the toes and that that method is adopted without being taught, is because of the heel on the shoe. With a highheeled shoe it is impossible to plantarflex the foot sufficiently to walk on the toes, and even with the heel but slightly higher than the sole, it is difficult to prevent it from coming in contact with the ground first. Fur- 
thermore, it is impossible to walk otherwise than heel and toe with the toes pointing outward, as is still so commonly done.

Before trying to walk properly one must learn to stand properly. One can not walk on the toes if one stands with the toes turned out. Conversely, it is much more difficult to walk on the heels if one stands with the feet parallel. If we follow the physiological action of walking from this standing position, it will be manifest that the toe should be presented to the ground slightly in advance of the heel. As the transverse plane of the body passes over the foot, the anterior muscles of the leg are contracting and flexing the tibia on the foot, the toes being held against the ground by the flexors, assisted by the weight of the body. Then, at the proper time, the gastrocnemius and the soleus lift the heel; the muscles passing behind the inner malleolus contract and the smaller toes grasp the ground, be it the earth or the sole of the shoe, the plantar muscles helping; the large toe is abducted - toward the middle line of the body rotated and held firmly against the ground and the mediotarsal joint is flexed and adducted - not by movement of the forefoot, but by the lifting upward and outward of the hind foot. The muscles passing behind the outer ankle contract and increase the height of the dome and prevent an exaggeration of the adducting action of the inner muscles. At the beginning of the contraction of the posterior muscles the anterior muscles relax. When the extreme of extension of the foot is reached, the line 
of the heads of the first and second metatarsals is at a right angle to the line of progression of the body; the arch of the foot is increased and the ankle thrown outward. Seen from behind, the toes are to the inner side of the foot, the heel being about in a line with the third or fourth toe. As the ground is spurned, and this spurning is done even when the foot is clothed, the knee is slightly flexed and the foot carried forward over the three outer toes by flexion of the thigh and by some rotation of the pelvis backward on the opposite side. Thus, as the foot is brought forward all of its muscles are relaxed and it is in the position of rest, the position of plantar flexion and inward rotation, and the toes are the nearest to the ground. An extraordinary effort will therefore be necessary to present the heel first; while, if the toes are to receive the first impulse of contact with the ground, the foot can be allowed simply to drop when the desired location is reached. To walk in this way with the toes turned outward would be impossible. This rest of the foot as it is brought forward may seem to be insignificant, but it is of great importance in preventing fatigue. Emphasis should be placed on the fact that it is only in walking as thus described, that control over the exact location of the footfall is to be obtained. In heel presentation it is impossible to recover or to change the footfall after the heel has touched the ground. No one would think of walking over a surface where each step had to be chosen with care, otherwise than on the toes. 


\section{CHAPTER III}

\section{EXAMINATION}

IN examining a foot some such procedure as the following is recommended. By using it in detail on every occasion, errors in diagnosis are less likely to occur.

Inspection.- This should begin with the patient's entrance into the examining-room. Is there a limp? Is the foot held in abduction? Is the clothing over the internal malleolus worn? Are the inner ankles prominent? When the patient stands are the feet parallel or divergent? Are the soles flat on the ground or do the toes turn upward? Are any of the joints, especially the first metatarsal-phalangeal, prominent through the shoe?

History.- Inspection will naturally be interrupted to take the history. Information should be sought regarding recent illnesses and injuries; the customary use of the feet; amount of standing, walking, dancing, and of any athletic sports. How long ago were the feet perfectly well? How did the present trouble begin? Has the patient ever experienced it before and entirely recovered from it? Is the complaint that of fatigue and weakness or of pain? Is it continuous or worse at times? If the latter, what seems to influence it; if use, what kind of use? How much use? Is it worse at 
night or in the morning? Are both feet affected? Was one affected before the other? Are both equally affected now? Family and personal history should be inquired into for any trace of tuberculosis, syphilis, gonorrhea, or rachitis. Rheumatism or gout may play an important part in a localized foot trouble. Careful inquiry should be made into any previous treatment, whether professional or that of some member of the laity, as this information will frequently help in determining what line of treatment the patient will be the most likely to follow where there is some choice permissible.

Both feet and legs, above the knees, should always be bared for examination in every instance. First inspect the shoes: locate the most worn parts on the soles and heels; is the upper stretched so as to overlap the sole or heel on either side? Is the inner side of the sole and heel on a straight line? Compare the height of the heel with that of the sole; is the center of the heel under the center of the weight-bearing part of the hind foot? Then examine the stockings: are they damp? are they pointed? Before their removal it had better be determined whether they constrict the toes. Note the color of the skin for signs of faulty blood supply. With the patient standing, notice the position of the toes: are they flat on the ground, flexed, hyperextended, parallel? Is there a hallux valgus? Does the forefoot appear to be flattened out, - extra wide? Is there a concavity or a bulging beneath the tuberosity of the scaphoid? Are the malleoli well defined? Does the outer one seem to be in its normal re- 
lation to the inner one or is it apparently advanced? When examined from behind, do the tendo Achillis run down vertically to the calcaneum or do they incline to one side? Are the normal depressions on either side of the heel-cord present? Does the heel spread out on all sides like an inverted mushroom? Ask the patient to rise on his toes; is it easily done? Does the dome heighten? Are the ankles thrown upwards and outwards? Can the patient invert the feet and stand on the outer borders?

Palpation.- Take one foot, the well foot first, if only one is complained of, on your knee in such a way that the entire leg is comfortable and relaxed. The surgeon's chair should be a few inches lower than the one upon which the patient is seated. Note by feeling whether the local temperature is normal. Search for evidences of uneven pressure or of friction, such as calluses and corns. If there are calluses under the forefoot, are they beneath each one of the five metatarsals or beneath only the middle three? Is there callous formation along the outer border of the foot, or around the margin of the heel? Is there a bunion over the first metatarsal-phalangeal joint? Are there ingrowing toenails? Determine the condition of the circulation of the foot. With a skin pencil mark the lower posterior angle of the internal malleolus, the tuberosity of the scaphoid, and the inferior external tuberosity on the head of the first metatarsal. A straight line connecting the first and last of these points should have the tuberosity of the 
scaphoid slightly above it. The tarso-metatarsal joint of the first toe is normally at mid-distance of the inner border of the foot and if measuring shows a lengthening of the posterior half, a lowering of the arch exists (Golding-Bird). If deformities of the toes are present, ascertain if they can be easily straightened by passive movements.

Hold the calcaneum firmly in one hand; with the tuberosity resting in the palm, grasp the bone with the thumb and fingers so as to prevent its moving, and with the other hand test the motion at the medio-tarsal joint. Then hold the leg above the ankle with one hand and grasping the foot about the medio-tarsal joint with the other, test inversion and eversion. Test the ankle joint last: in so doing do not let flexion and extension at the medio-tarsal joint deceive you into attributing it to the ankle joint, so grasp the foot that the os calcis moves synchronously with the metatarsals. Care must also be taken that the foot is moved in the vertical plane of the leg, otherwise abduction in dorsal flexion will exaggerate the true angle of flexion. The range of active movements of all the joints, with the foot in the resting position, should be determined. Osgood tests the muscles in the performance of the various movements; and, after determining their relative strength, prescribes exercises, if their need is indicated, to restore the normal muscular balance.

Pain.- Though pain is of no more importance in making a diagnosis of an abnormal or diseased foot than 
it is in morbid conditions elsewhere in the body, it is often of great significance in making a differential diagnosis, and the painful spots should always be definitely located. Pain caused by pressure over a diseased or injured bone is usually more circumscribed and elicited more easily and definitely than the pain from pressure on a strained or ruptured muscle or ligament. A strained or ruptured muscle or ligament is always painful if stretched. This stretching is produced in the ligament only by separating the ends, but in a muscle a contraction will produce it.

Definite pain upon pressure over the body of the os calcis or of the first metatarsal is generally due to disease or injury to those bones. Pronounced pain over the peroneal tubercle on the external surface of the os calcis, is due, according to Goldthwait, to a tension of the synovial sheath of the peroneal tendon dragging it away from its attachments to this tubercle.

Pain about the external malleolus in cases of everted feet, is due to a crowding of the tissues against the external malleolus from malposition of the tarsus, according to Golding-Bird. The pain about the inner side of the medio-tarsal joint may be due to an inflammatory condition of this joint, or to strain. 


\section{CHAPTER IV}

SHAFFER'S FOOT (NON-DEFORMING CLUBFOOT). WEAK-FOOT. FLAT-FOOT

THE condition of a shortened heel-cord has been noted by several writers and more or less importance has been attached to it. Thus Adams, "Club-Foot": "A certain amount of lameness accompanied by inability to walk even a moderate distance without fatigue, follows this condition of right-angled contraction of the tendo Achillis and in consequence of the limited flexion of the ankle joint the stride is necessarily shortened; for the same reason, also, the feet in the act of progression are turned outward with a twisting movement. It is difficult to recognize the condition from the absence of deformity."

And thus Holmes Coote, "On Joint Diseases": "In many cases of talipes valgus the tendo Achillis is so tight that the foot can not be raised beyond a right angle to the leg. In such cases a cure can not be effected without the operation of tenotomy." Tubby, in his recent work devotes five pages to "This simple and seldom described condition of contraction." Shaffer, however, was the first to describe its mechanics, or rather its interference with the normal mechanism, its clinical signs and symptoms, its complications, and its treatment, for which he 
invented an ingenious apparatus which has been in successful use for over a quarter of a century.

\section{SHAFFER'S FOOT}

The chief characteristic of this condition is a shortening of the gastrocnemius and of the soleus. This

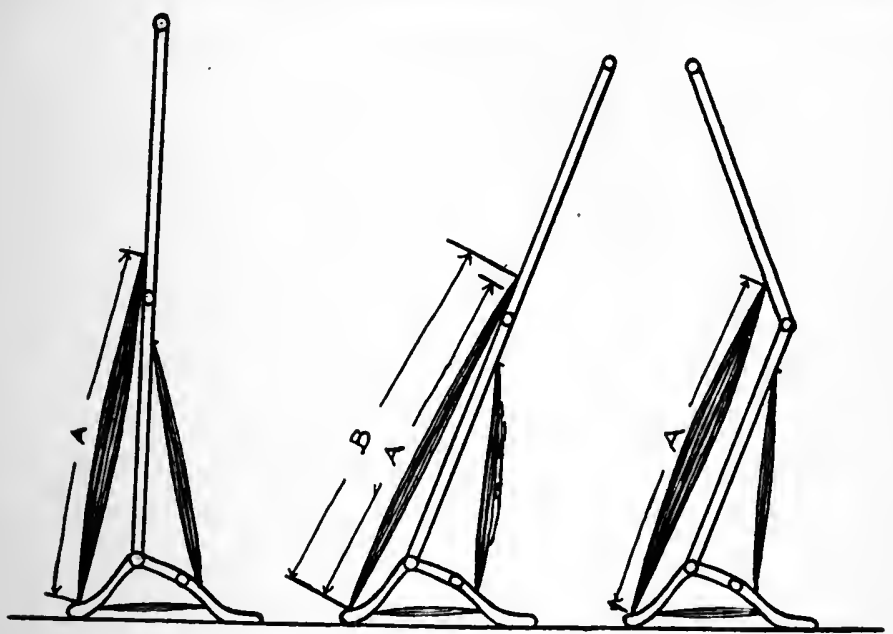

Figs. 12-I4. Effect of a Shortened Gastrocnemius

Fig. $I z$ is a schematic drawing representing the thigh, leg and foot, with the knee, ankle and medio-tarsal joints and the gastrocnemius and anterior muscles and the plantar fascia.

Fig. 13 shows the normal lengthening of the gastrocnemius when the anterior tibial muscles contract, flexing the leg upon the foot. The length of the gastrocnemius when the foot is at a right angle, $A$, has been increased to $B$.

Fig. I4 illustrates how, by bending the knee, flexion of the leg may take place without lengthening of the gastrocnemius.

shortening is not enough to produce the deformity of equinus but limits dorsal flexion. Normally, the foot can be brought up in the line of the leg, until the plantar surface forms an angle with the leg of 75 
degrees. In this condition, however, this angle is 90 or 100 degrees. As a result, when the foot is flat on the ground and the tibia is being flexed on the foot, the action is blocked at the ankle before the movement is completed. What takes place may be readily understood by reference to the diagrams. Fig. I2 represents the normal position of the leg and foot, showing the anterior and posterior muscles and the plantar fascia. Fig. I3 illustrates their positions as the body is brought forward and before the heel is raised to propel the body forward upon the other foot. The anterior muscles are contracting and flexing the leg on the ankle. The posterior muscles are relaxed.

Fig. I4 illustrates what may take place if the posterior muscles are contracted and prevent flexion at the ankle: the bending of the knee brings the heads of the gastrocnemius nearer to its insertion, thus providing for the increased ankle movement.

Fig I 5 illustrates how, if the heel is raised, this ankle movement may be increased; the blocked movement being compensated, without bending the knee.

Fig I6 shows where the strain will be felt if the knee is not bent and the heel is not raised and the anterior muscles are strongly contracted in an endeavor to flex the tibia. The gastrocnemius will be stretched, and strain will be felt at the medio-tarsal joint, in an endeavor to over-extend it, and there will be strain of the plantar tissues, which prevent, by their bow-string action, abnormal extension of this joint. The dotted line shows the 
normal position of this joint. This strain will be due not only to the contraction of the anterior muscles but also to the weight of the body in an inverse proportion to the amount of contraction of the gastrocnemius.

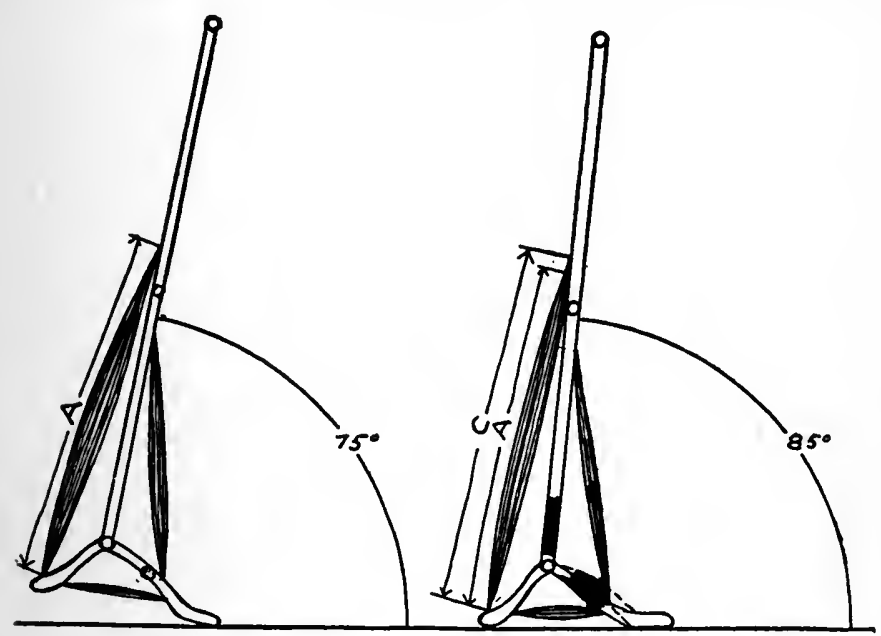

Figs. 15-i6. EFfect of a Shortened Gastrocnemius

Fig. 15. In this figure the knee is not flexed and the gastrocnemius is not lengthened, but by raising the heel the leg is brought forward to form an angle of 75 degrees with the ground.

Fig. I6 demonstrates the force brought to bear toward extension of the medio-tarsal joint when the gastrocnemius allows of slight dorsal flexion, to 85 degrees in this figure, and the consequent strain on the plantar tissues with lowering of the dome and lengthening of the foot.

Thus, if the shortened gastrocnemius permits of the tibia's being flexed Io degrees beyond the vertical, the weight of the body, acting not so much as superimposed weight as power to a long lever arm, will strain the medio-tarsal joint and the plantar tissues much more 
than if the flexion of the tibia is nil, is stopped at a right angle.

Consequences.-Therefore the consequences of this condition will depend on the extent of the contraction and upon the use to which the abnormal mechanism is put. With flexion limited to a right-angle, accommodation may be made by bending the knee or by raising the heel during attempted flexion of the leg, and by taking short steps. Usually patients who complain that they can not take long steps or can not wear low-heeled shoes will be found to have this limitation of motion at the ankle. In these instances, a slight cavus, a high arch, will usually be present and the foot will be shorter than normal from a slight contraction of the plantar fascia.

Symptoms. - In adults the complaint for which relief is sought when dorsal flexion is limited to Ioo or 90 degrees, is pain in the calves, about the knees, often in front of the knee, or, more rarely, up the thighs, sometimes extending to the buttocks and the lumbar region. This pain is entirely relieved by rest and brought on by walking. It is due to muscular, especially gastrocnemic, strain. It may be so severe as to prevent walking more than a few steps without rest. Any exercise which demands dorsal flexion will be found to be difficult or impossible: walking down stairs will sometimes be done backward, as the necessity of flexing the leg on the foot in descending is impossible for these patients without bending the knee or raising the heel, and in so doing there is a tendency to pitch one head foremost down the 
rest of the flight. There may be a history of frequent sprains of the ankle due to the instability of the joint. In a patient whom I saw in consultation there was com-
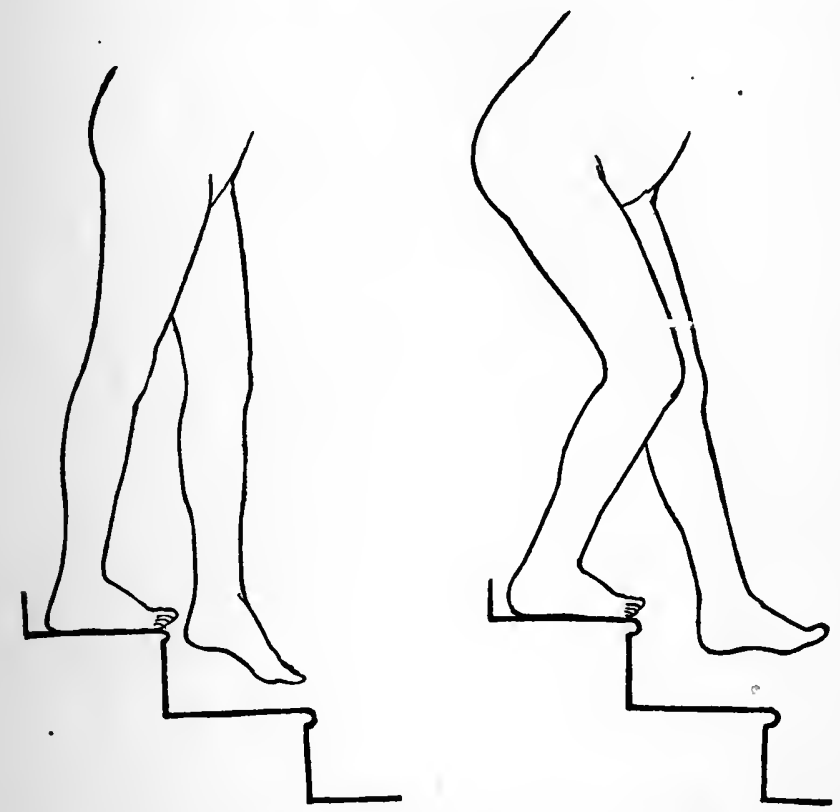

Figs. 17-18. Illustrating the Difficulty in Descending Stairs WHEN DorSal-Flexion of tHE FoOT IS LIMITED

Fig. 17. The right leg cannot be flexed on the foot so as to permit the left foot to reach the next step.

Fig. 18. This difficulty might be overcome by flexing the knee, but this would be most awkward.

plaint of a feeling of weakness of the knees, as though, when standing he felt some force pressing them forward, trying to flex them. There was no particular pain. Examination failed to reveal anything abnormal with the 
knees or feet except a limitation of dorsal flexion to about 90 degrees. In this case the strain of the short-gastrocnemius was felt in its tendency to flex the knee, and not at all in the ankle or foot.
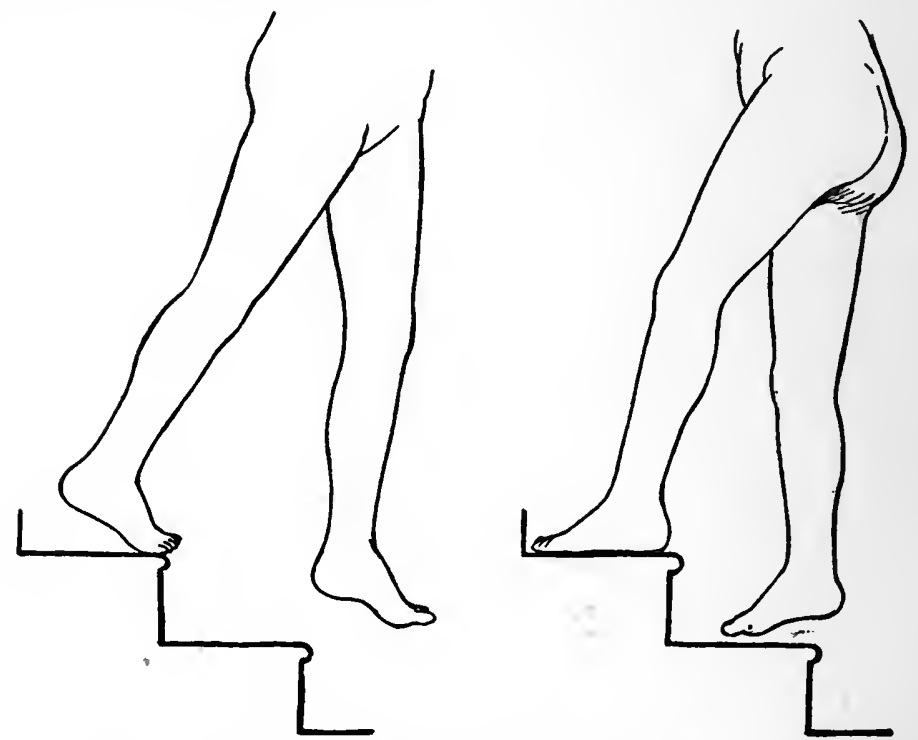

Figs. 19-20. Illustrating the Difficulty in Descending Stairs WHEN DORSAL-Flexion of the FoOt IS Limited

Fig. 19. By raising the heel the shortening of the muscle may be compensated, but this endangers one to a pitch head foremost down the rest of the flight.

Fig. 20 shows how the obstacle of this limited motion is frequently surmounted: descent is made backward, dorsal-flexion then being unnecessary.

In children, consultation is sought on account of the awkward gait and the clumsiness. The child is constantly stumbling and falling.

On the other hand, with a contraction less marked 
wherein the tibia can flex on the foot to Io degrees or so beyond the vertical, the added weight of the body to the strain upon the medio-tarsal joint and upon the entire dome of the foot, is much more serious in its deforming effects. The contraction itself is not so incapacitating as where the contraction is greater; the stride is not so limited and the muscle-strain is not so great. Pain may be felt only after unusual exertion and is more likely to be limited in extent, and is more of a tired feeling than of acute suffering. But strain toward increased dorsal flexion at the medio-tarsal joint is much more pronounced than when the ankle flexion is more limited, and the plantar tissues are now the subject of severe strain at each step. In an unconscious attempt to relieve this, the foot is abducted, as by so doing the limited ankle flexion is compensated to some extent, but this vicious attitude hastens the oncoming deformities.

Thus it may be understood how this condition may cause muscular pain from stretching of the calf muscles, or pain about the knee from the strain of supporting the body with the knee slightly flexed, or pain at the astragalo-scaphoid articulation from strain of overextension, or over the tuberosities of the os calcis from a periostitis set up by the strain on the plantar fascia. Also one can understand why the toes may be turned decidedly inwards when the dorsal flexion is so limited as to approach an equinus deformity; and why, with less limitation, the foot is everted even while the dome is as yet normal. The loss of firm support at the end of each step accounts 
for the weak ankle and frequent sprains which occur in some cases. Then, if the condition persists, the reason is understood for the formation of calluses under the five metatarsal heads, the flattening of the anterior arch, and finally the condition of flat-foot.

Etiology.-The etiology of Shaffer's foot must be sought for in anything that will cause slight shortening of the gastrocnemius. Anything that will cause a prolonged shortening of the calf muscles must lead to a more or less permanent condition: such as prolonged illness during which the patient's foot has not been supported but allowed to remain in the foot-drop attitude. Possibly some nervous conditions may keep the posterior muscles from being perfectly stretched. Tubby assigns an imperfect recovery of the anterior muscles from an attack of anterior poliomyelitis as a cause. It frequently follows attacks of rheumatism and of sciatica.

Treatment.- Treatment consists in lengthening the gastrocnemius. As a very slight lengthening is all that is necessary, the tendo Achillis being attached to so short a lever arm, an operation is searcely demanded.

Traction Shoes.- Shaffer's traction shoe is a most efficient apparatus to accomplish the desired end. In some apparatuses devised for this purpose, notably, Scarpa's shoe, the mechanics of the foot have not been correctly met. The heel has been pulled directly forward and account has not been taken of its moving about the axis of the ankle joint, and therefore, during dorsal flexion, moving in an arc, downward and forward. In 
Shaffer's traction shoe, after the application to the foot and the securing of the astragalar strap across the instep, the traction, by means of the ratchet upon the heel-strap, draws the heel downward as well as forward. Every ounce of force is exerted in the best mechanical direction, and, when properly used, is felt only in the tendo Achil-

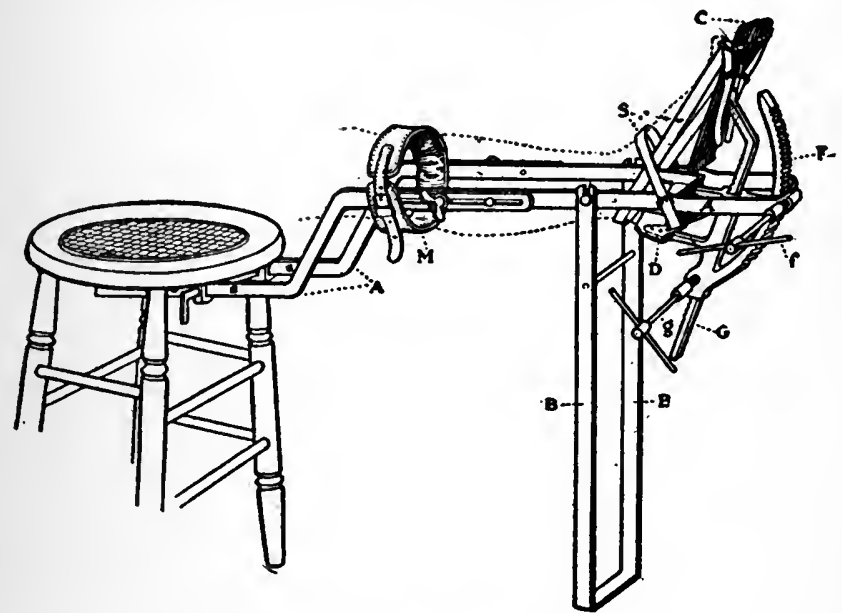

Fig. 2I. Author's Traction Shoe

Comprising the essentials of Shaffer's Traction Shoe with the addition of the arc-ratchet. The vertical ratchet is seldom used and is not necessary.

lis and its muscles. Ten minutes of daily application of intermittent traction will, except in the most resistant cases, show an increase in the range of dorsal flexion after eight or ten treatments.

The writer's traction shoe has added a ratchet, curved to correspond with an arc drawn from the axis of the ankle, which moves the foot as a whole, and therefore 
each component part, upon the radii from this axis as a center. This machine is secured to a chair or stool upon which the patient sits. After the shoe is properly adjusted, all the force desired can be applied to the contracted tissues.

The machine consists of two lateral bars, a stand, and a foot-piece. The lateral bars A, A, are joined at their distal ends in a U-shaped piece. Their proximal ends are bent so as to pass beneath the chair where they are secured by a draw-pin passing through sockets screwed to the bottom of the chair-seat. The continuity of the lateral bars is interrupted just below the calf-band $M$, and the ends overlapping, are held by thumb screws. Thus these bars may be shortened or lengthened as desired. The stand B, B, supports the machine. The foot-piece consists of a foot-plate $\mathrm{C}$, and a heel-cup $\mathrm{D}$. The foot-plate is joined to the heel-cup by the ratchetbar $G$ passing through the sheath $E$, which is secured to the bottom of the heel-cup. The entire foot-piece is riveted to the lateral bars at $j, j$, and by means of the ratchet $F$, can be moved about the axis passing through $j, j$. The act of the ratchet $F$, is constructed on the radius $\mathrm{jF}$. To the bottom of the foot-plate are riveted two buckles h, h, which secure the heel-strap. This strap passes behind the heel, forward on both sides of the foot, over the hangers $i$, $i$, projecting upward from the front of the foot-plate, and backward to the buckles. On each side of the heel-cup are rowels, $k, k$, through which the astragalar strap, $\mathrm{S}$, is roved. The strap crosses over 
the ankle, opposite the head of the astragalus, is doubled on itself and buckled.

The method of application is as follows: The patient

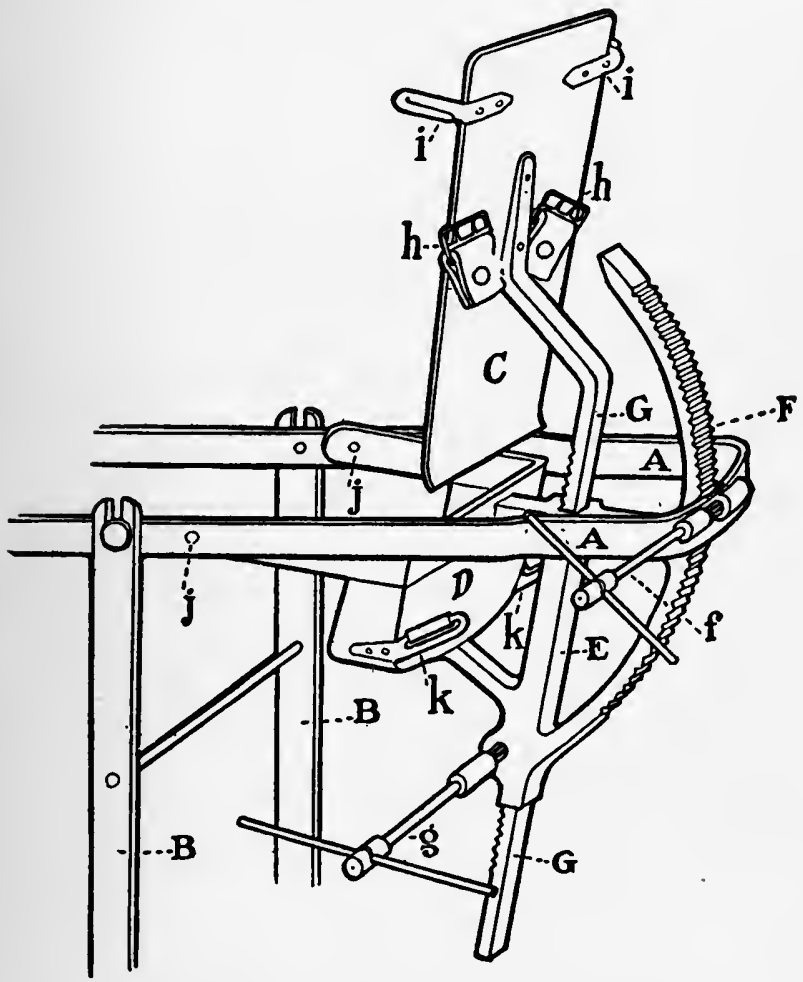

Fig. 22. Author's Traction Shoe

Illustrating in detail its various parts, which are described in the text.

is seated in the chair, the heel placed in the heel-cup, D, and the lateral bars $A, A$, adjusted by means of the thumb screws to the exact length of the limb with the 


\section{DISEASES AND DEFORMITIES OF THE FOOT}

leg fully extended. It is necessary that the axis of the ankle-joint pass through the axis of the foot-piece, $j, j$. The heel-strap makes this adjustment possible. While fitting the apparatus the foot-piece should be plantar flexed to an angle affording an easy position for the foot. The last thing to be done is to fasten the strap of the calf-band, thus preventing the knee from flexing as the strain comes on the gastrocnemius. Traction may now be started. Using the key, $f$, the ratchet, $F$, is made to pass forward and around the axis $\mathbf{j}, \mathbf{j}$. Everything on that foot-piece will move through arcs the radii of which extend from the common center. The foot is thus moved forcibly about the ankle-joint. When tautness of the tendo Achillis, as ascertained by palpation and by the sensations of the patient, warns one that the limit of flexion is reached, the astragalar strap is applied and a momentary hyperextension produced by means of the antero-posterior ratchet, G. This is the part which is exactly similar to Shaffer's traction shoe.

After one or two treatments with either of these apparatuses, the patient will usually express himself as noticing an improvement. The pain and discomfort is lessened, the foot feels "lighter" and walking is easier. The patient should not be discharged until the normal extent of dorsal flexion has been obtained and all faulty postures, in walking and standing, corrected.

Operation.- Tenotomy of the tendo Achillis is done by some surgeons for this condition but is not advocated by many. Lengthening of the tendon by open 
operation, as recommended by Tubby, is much more exact and can not result in a calcaneous deformity, which that surgeon believes to be a danger of simple tenotomy. By the open method, the tendon is divided half way

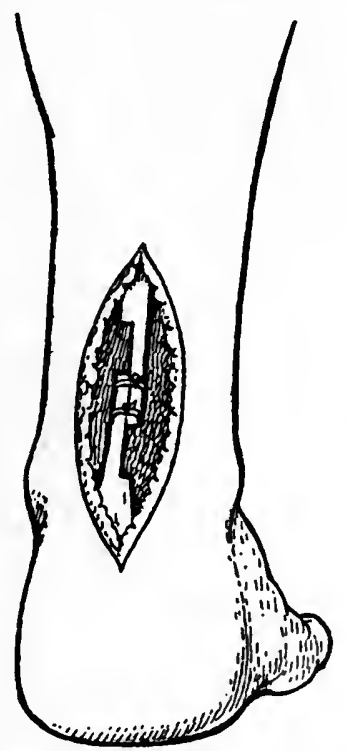

Fig. 23. Lengthening the Tendo Achinlis

After making the two incisions which extend half way across the tendon from opposite sides and at a suitable distance, one above the other, and then connecting them with a longitudinal, central incision, the foot is placed in the corrected position and the overlapping parts sutured.

through its width, by two incisions from opposite sides, one being two inches or more above the other. These two partial divisions are then united by a vertical central incision which joins their innermost ends at right angles. The foot is next placed in its proper position of full 
dorsal flexion, the divided parts of the tendon sliding on each other leaving a gap at each transverse incision but no gap extending clear across the tendon, and it is then sutured. The foot is put up in plaster-of-Paris and should be retained for three weeks in this position, either with the plaster dressing or by a brace.

Although these cases present no deformities of the dome of the foot, many are treated, through faulty diagnosis, as though they were weak- or flat-foot, with strapping, wedge soles, exercises and so forth, and even with braces, and are sometimes cured by these means through the correction of attitudes which aggravate the condition and through forcing an active stretching of the gastrocnemius.

\section{WEAK-FOOT}

Two Classes. - Weak-foot we would divide into two classes: the ligamentous and the muscular. They may exist together but, at least in the early stages, the weakness may be predominantly either ligamentous or muscular. The chief reason for making the differentiation, where possible, is to meet the specific indications in treatment and to avoid the prescribing of unnecessary and possibly injurious remedies.

Characteristics.- In either form the patient stands and walks with the feet abducted. There is usually a listing of the foot to the inner side, which is noticeable with the shoes on or off. The soles of the shoes will show more wear along the inner side. The counter on 
the inner side may overrun the heel; and the leather between the heel and toe of the shoe, along the inner side, may be drawn into furrows or wrinkles lying in a more or less longitudinal direction. These conditions come
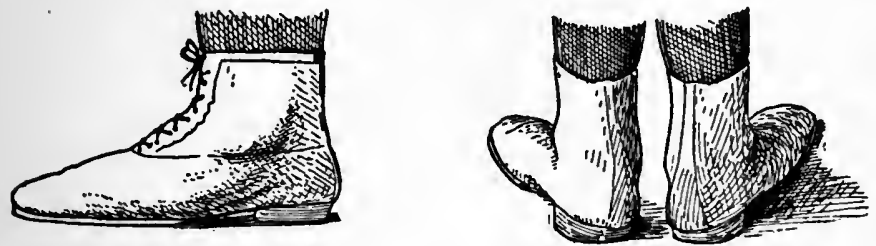

Figs. 24-25. Adducted ANd Everted Feet

In Fig. 24 note bulging along the inner border and the prominent internal malleolus.

In Fig. 25 note that the back seam of the shoe is directed downward and inward, instead of straight downward, and that the inner border of heels are more worn than the outer. The leather over the internal malleoli may be worn or may be dirty, showing interference as the feet pass one another in walking.

from the strain on this part of the shoe, produced by the abduction of the forefoot. The leather over the inner ankles may be worn from the interference due to walking with the forefoot abducted.

While the patient is standing in bare feet, the earliest evidence of weak-foot is to be seen in the altered direction of the tendo Achillis. Its direction will be downward and outward instead of directly downward. It should be borne in mind that this sign is also present when there is a shortened heel-cord without any marked weakness. The prominence of the internal malleolus will also be increased. If there is much abduction of the forefoot, the altered direction of the long axis of the foot will give the appearance of the external malleolus 
being advanced to a position more nearly in line with the internal malleolus. In marked flat-foot it will apparently be even in advance of the internal malleolus. In neither case has any actual change taken place in the relation of

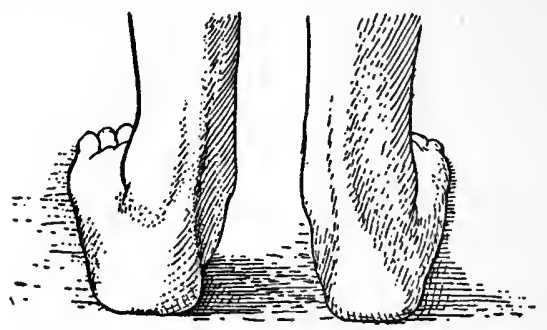

Fig. 26. Abducted and Everted FeEt

Seen from behind. Note altered direction of the tendo Achillis, valgus of the ankle and the spreading out of the heel.

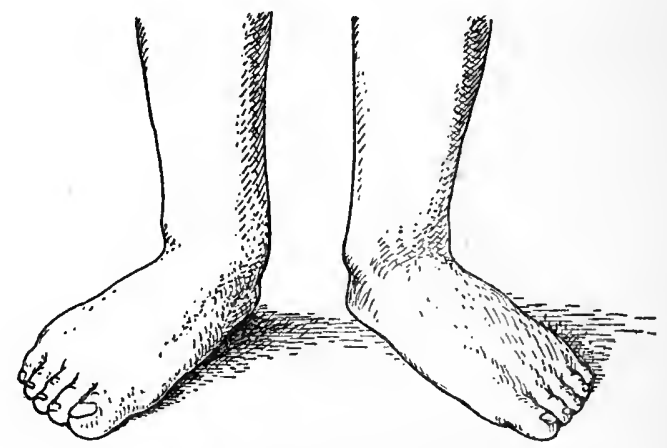

Fig. 27. Abducted and Everted Feet

Seen from in front.

the tibia to the fibula. The amount of bulging on the inner side of the ankle will depend upon the degree of weakness of the muscles and of the ligaments. If the former are mostly at fault, the deformity will be little 
or not at all changed whether the patient stands or walks. On the other hand, if the greater weakness lies in the ligaments, the deformity will be obliterated whenever the muscles are brought into play, as when the patient walks or stands on tip-toe.

Pain is more apt to be present and to be more severe
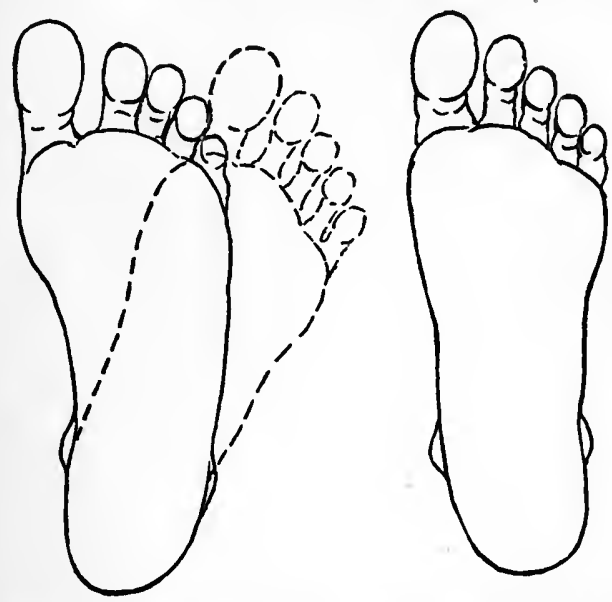

Figs. 28-29. Advancement of the External Malleolus

Fig. 28 shows the normal relative position of the external to the internal malleolus. If the fore-foot is abducted as shown in the dotted lines and then the foot as a whole turned inwards by rotating the leg, as has been done in Fig. 29, the external malleolus will apparently be displaced forward.

in the muscular form. The feet tire sooner than they should. There may be some synovitis: this may be evident in front of the external malleolus, over the subastragaloid and over the calcaneo-cuboid joints, and perhaps less frequently on the inner side below the malleolus and over the astragalo-scaphoid joint. 
If there is synovitis of the ankle joint, bulging will be evident between the tendons on the front of the ankle and on each side of the tendo Achillis.

Improper use of the foot must result in a weakening of its muscles and ligaments and, as improper use in both standing and walking is more common than proper use, those whose occupation demands their being a great deal on their feet are the greatest sufferers. Osgood $*$ has made a study of the comparative strengths of the adductors and abductors of the foot and the variations in this ratio in the normal and in "foot-strain." He found the ratio of the adductor pull to the abductor pull in normal feet to be Io to 8.2 in favor of the adductors. In pronated feet without symptoms io to I0.5 in favor of the abductors. In pronated feet with symptoms ro to ro.8 in favor of the abductors. In acute feet with valgus Io to $\mathbf{I} 2.2$ in favor of the abductors.

Weak-foot is common in children who have grown rapidly and in whom the muscular development has not kept pace with the bony growth. This inequality of growth is made more evident by severe sickness, for the bones seem to take all the nourishment they need regardless of the condition of the other parts of the body.

The ligamentous variety of weak-foot is frequently associated with rachitis. The musculature may or may not be normal. The ligaments of other joints may be lax; it may be possible to extend the elbow beyond its nornual limitation; there may be lateral movement of the

* Boston Medical and Surgical Journal, March I3, I9I3. 
knee when it is in the extended position, and postural scoliosis may exist.

Results of Non-treatment.- If weak-foot is permitted to exist without protection, the unnatural strain, due to the abduction and eversion, together with the absence of normal functionating, increases the weakness. In this position of the foot, all movements at the mediotarsal joint and at the sub-astragaloid become very painful, and to avoid this muscular spasm develops, first marked in the peroneals, in an effort at immobilization. Further use, without treatment, is accompanied by increased muscular spasm, resulting in rigidity, followed by a lowering of the dome of the foot, by bony changes, and by flat-foot as a final result.

Treatment.-This is taken up with the treatment of flat-foot.

\section{FLAT-FOOT}

The diagnosis of flat-foot, if the condition of weakfoot is recognized as an entity, rests upon the fixed reduction or obliteration of the dome of the foot. Walsham recognizes four degrees of deformity; oncoming, pronounced, rigid, and osseous. As weak-foot, according to our definition, becomes a flat-foot only when complete, voluntary restoration of the normal relations of the bones becomes impossible, and as this restoration in the early stages is prevented only by spasm, it would seem to be simpler to have but two stages of flat-foot: the spasmodic and the osseous. The former (as it is always 
progressive if the etiological factors persist) could be divided into the oncoming, which is more or less easily corrected by manipulation; and the rigid, where nothing short of great force, necessitating an anesthetic, will allow of restoration of the dome, but where no marked bony changes have as yet followed. The osseous includes all cases which are irreducible without removal of bony obstruction.

Many qualifying and descriptive terms may be used which are an aid in history-taking and provide a more or less clear description of the case, either as to an etiological factor or as to the condition, such as traumatic, paralytic, inflammatory, painful, etc., but it is unnecessary to classify under any such extended system.

Osseous Flat-Foot.- This is the result of unre$\{$ lieved eversion and abduction and may or may not be a painful condition. If firmly established with practically no movement in the intra-tarsal joints, the pain associated with it will arise from uneven plantar pressure. All the finer accommodating movements being lost, the bones can not be shifted to accommodate themselves to alterations in the plane of the body containing the center of gravity, nor to changes in the surfaces upon which the foot rests, and calluses will form at the spots receiving the greatest weight, the pain depending to a great extent on the condition of these calluses. This foot, the veritable flat-foot, has lost all the functions of a normal foot except that of support, and the supporting is done in an inanimate rather than in a physiological way. Yet 
the possessor of such a foot may have so accommodated himself to the condition as to be unaware of the extent of his deformity. Many cases of the most marked type exist, in which pain was never severe.

Pathology.- In these conditions of the foot the pathology is limited to stretching of some muscles and ligaments and to contracting of others, except in that of osseous flat-foot. Here there are certain bony changes which should be recognized in planning treatment. The eversion, the rotation on the sub-astragaloid axis, has so altered the position of the os calcis that its external inferior tubercle is lifted from the supporting surface and an articulation may be formed between the external surface of the bone and the external malleolus. The anterior part of the os calcis has been abducted. The external arch, with the entire dome, has been lowered and the eversion has so rotated the bones that the cuboid is on about the same horizontal plane as the upper part of the head of the astragalus. The superior faces of the internal cuneiform and of the first metatarsal and the upper part of the head of the astragalus, look inward. It is this rotation of the foot which throws the external malleolus into such prominence and which makes the internal malleolus less so. It is the abduction of the forefoot which gives the appearance of the external malleolus having been moved to a position in advance of its normal one. The scaphoid may be greatly altered in shape (Walsham. Lorenz) and its tuberosity look downward instead of inward, thus giving an altered direction 
to the tibialis posticus. The greater part of the inner surface of the head of the astragalus is uncovered as it is depressed and rotated inward while the scaphoid, in relation to it, is displaced upward and outward. Exuberant bone may be present on what is now the outer and upper part of the head of the astragalus and also about the cuboidal facet of the os calcis.

\section{TREATMENT OF WEAK-FOOT AND FLAT-FOOT}

Our object is reduction of deformities, whether easily reducible or fixed, and the restoration of normal functions. Usually these two processes, reducing deformity and restoring function, can go on together, but under no circumstances should a cure be pronounced until both results are obtained.

Weak-Foot.-If a weak-foot exists, wherein the foot at rest appears about normal but upon weight-bearing assumes an abducted and flattened appearance, with more or less valgus, means must be taken to preserve the normal position of the foot while it is in use and steps taken at the same time to restore strength to the weakened muscles and ligaments.

Mild Cases.-Especially in cases of the ligamentous variety, nothing may be needed to hold the foot in the corrected position beyond the shaping of the heels and soles of the shoes in such a way that the foot is held inverted when weight-bearing, as inversion alone may correct the abduction. This is done by having the heel and sole made wedge-shaped, with the thickest part of 
the wedge on the inner side of the foot and the edge of the wedge on the outer side. The amount of thickness of the heel and sole along the inner side will be found to vary in different cases, but the correct amount can be determined by examining the feet from behind when a wedge is inserted under the foot. In mild cases f an eighth of an inch is all that is required, but more fre-

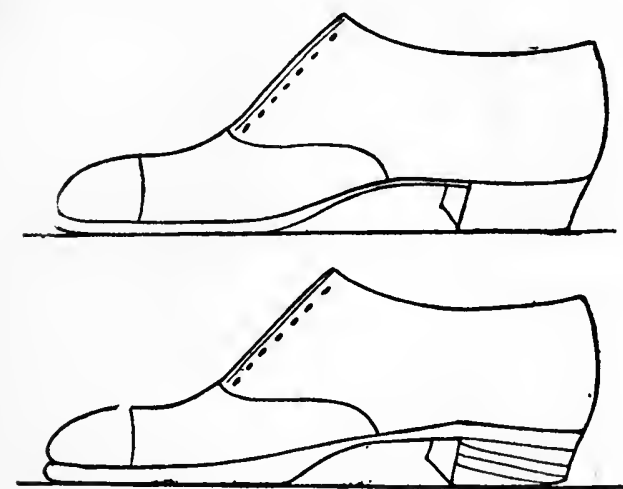

Figs. 30-31. A Shoe Before and After the Insertion of Wedges

Wedges are placed at the anterior and inner part of the heel and at the posterior and inner part of the sole for the purpose of correcting faulty balance. Figs. 30 and 31 show a shoe before and after the insertion of these wedges.

quently a quarter or even half of an inch will be needed. The ideal way of making the wedge is to construct it so that the sole and heel will correspond to segments of the dome formed when the two feet are placed together. That is, the wedge added to the heel will be highest at the internal corner and slope downward toward both the external border and the back; and the wedge added to the sole will be the highest at the internal and back part 
and from there slope downward both externally and forward.

The Beely-heel extends inward to a marked degree and thus offers an irresistible obstacle to eversion - when the most marked deformity is in the sub-astragaloid joint, this inward extension of the heel should be prescribed.
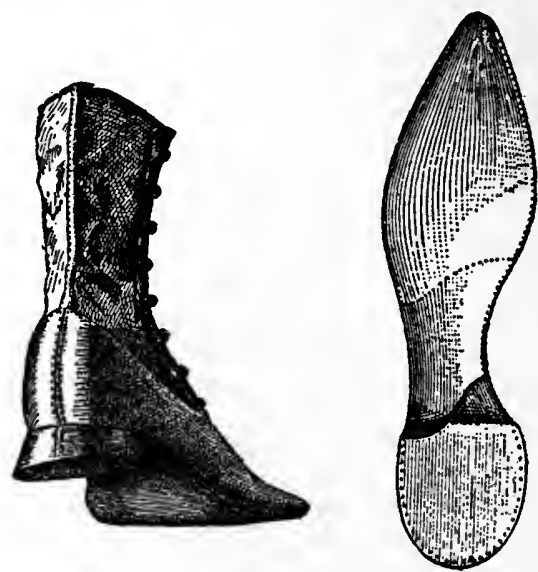

Figs. 32-33. InVERTINg HeEL

The extension of the heel inwards prevents eversion of the foot. This illustration is after Beely, taken from Handbuch der Orthopadischen Chirurgie. The toe of the shoe is not to be recommended.

By extending the heel forward so that it gives secure support as far forward as the astragalo-scaphoid joint the adduction may be more securely held. This may be done by the Thomas heel which extends forward threequarters of an inch farther on the inner than on the outer half. Adhesive plaster strapping, such as is described for the spasmodic condition, may be applied until 
the patient becomes accustomed to the new position; until the muscular sense has become re-educated.

Exercises.-The object of these is to strengthen the muscles and ligaments. There are many athletic sports which will do all the exercising necessary, at least in the mild cases, and for young people they will be found to be a much more welcome prescription than definite foot-exercises to be carried out in the bed-chamber. As such may be mentioned, tennis, running, jumping, cycling, ball, and in fact any game which necessitates one's springing about on the toes. Roller-skating is injurious because the feet are abducted. Dancing is very beneficial for weak-foot.

Specific exercises will, however, frequently have to be ordered. There are three sets which will be very useful in most cases: with the feet forming a square, slowly raising one's self up on the toes and slowly returning to the heels; walking about the room on the outer borders of the feet; using the toes to pick up articles and also strongly separating the toes. In the first exercise, care should be taken that the feet are in the proper position, parallel and forming the two sides of a square. The exercise must be done very slowly so that no help is given to the muscles by a swinging motion of the body or by allowing gravity to return the heels quickly to the ground. It will be found to be a great help in obtaining a cure if the patient is taught the habit of rising on the toes while the shoes are on. This will not take the place of the exercise as done bare-footed, but 
if the patient is instructed never to stand for more than a minute or two without rising on tip-toe and once in a

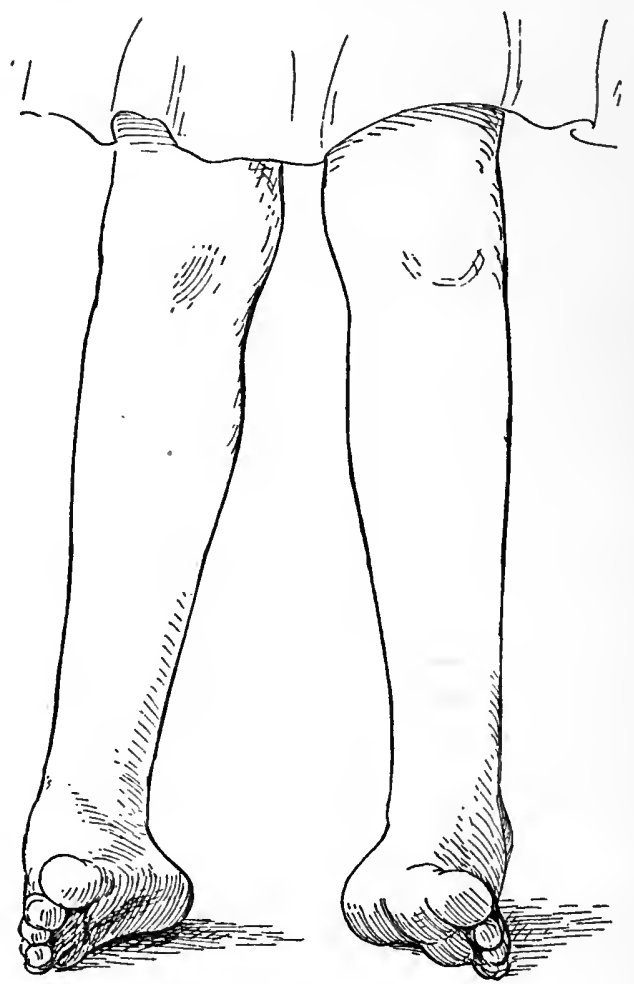

FIg. 34. AN Exercise For WEAK-FEeT

Walking about the bedroom on the outer borders of the feet tends to overcome abduction and eversion and strengthens the muscles of adduction and of inversion. This exercise is recommended by. Gibney.

while to rest on the outer border of the shoes, it will give a valuable rest to the ligaments. A most valuable 
exercise, which can not be done, though, without assistance, is rotation of the forefoot. The patient is seated with the leg resting on the physician's knee. The physician holds the heel securely in one hand, pointing the index finger of the other hand downward, with its tip just in front of, dorsal to, the second toe; the patient

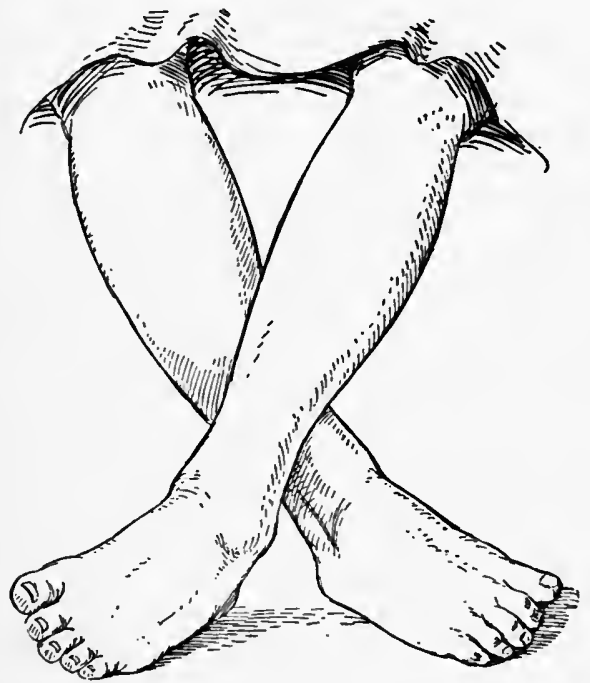

Fig. 35. The Feet at Rest

When sitting, the legs should be crossed and the feet allowed to rest on their external borders. This position puts all the structures at rest.

moves the forefoot, first in adduction and then through flexion, abduction and extension, in such a way as to carry the forefoot in circumduction around the physician's finger, which is held steadily in the one position. The exercises for the toes are directed toward increasing the 
strength of the smaller muscles and thereby strengthening the anterior arch, which will generally be weakened with the rest of the dome. When sitting, patients with a tendency to abduction and eversion should cross their legs in such a way that the feet will rest upon their external borders. This will rest all the foot-structures and be of inestimable value in preventing deformities.

These exercises will, of course, be impossible unless the foot is flexible.

Arch Supporters.-There is always associated in the minds of both the laity and the profession, in connection with the treatment of all these foot-troubles, the question of arch supporters. Few patients (complaining of their feet), come to the orthopedic surgeon, who have not worn at least one variety of the many to be found on the market. And, although the practice of unprofessional prescribing of arch supporters should be unreservedly condemned, for much the same reason as is counter-prescribing of drugs, I should not be surprised if shoemakers and salesmen of these articles had cured as many painful feet as have physicians. If this is true it is not because of any superior intelligence on the part of the cobbler or clerk, but because so few physicians possess any better knowledge of the etiology and pathology of these conditions than do the knights of the last, and the latter have a much greater opportunity to have a "try" at treatment; and that, too, usually before the condition has become what the patient would call serious.

It is a fact that many physicians, and not a few of 
them surgeons, are to-day sending their patients who complain of painful feet to the brace-maker or the shoemaker with orders for arch supporters. The object sought by all these people concerned is to supply something that will directly support the longitudinal arch, which often feels weak and may have the appearance of being depressed. This object is never obtained, but the relief of symptoms and even a cure may nevertheless result. If something, anything, is placed in the shoe in the neighborhood of the astragalo-scaphoid joint, which will force the patient instinctively to adduct the forefoot so as to avoid pressure against that object, it may lead to a cure. Adduction of the forefoot, by causing a concavity of the otherwise straight, or even convex, inner border, makes room for the foreign body, the supporter, and relieves pressure from it; and this adduction, the strong position of the foot, relieves the strain which is causing the pain and weakness. Likewise certain cases may be cured by having a supporter so shaped as to act like an incline-plane, causing an inversion of the foot; and if the forward part inclines downward, forward, and outward it may help to produce adduction as well. When these supporters, braces, or plates, as they are variously called, have any curative effect it must be their helping directly by force or indirectly by exciting muscular action, in keeping the foot adducted and inverted. They can be of no use and will cause greater pain if the foot is not perfectly flexible; and if the foot is flexible they usually do no more than postpone the time when expert treat- 
ment will become necessary, unless accidentally something is done to strengthen the weakened muscles and ligaments \while the supporter is being worn. Of themselves they tend to injure the foot by limiting its intrinsic movements and therefore weakening its structures. If at the beginning of foot-trouble, a correct diagnosis were made and proper remedial measures taken, there would be comparatively few cases which would need any brace of any description.

When for any reason the patient can not be taught to stand and walk properly,- and there are many such, especially among charity patients,- a correctly constructed brace may be applied and worn with great profit for some months.

Whitman's Brace.-This is designed not as a retentive apparatus alone but as a corrective one also. It is supposed to reëducate the muscles of the foot. When one stops to consider what a revolution it is, in the use of all the structures of the foot, to change from the abducted, everted position to that of adduction and inversion it is easy to appreciate that care and attention and intelligence as well are necessary, and many have not these to give even in forwarding their own cure. With a Whitman's brace it is impossible for the foot to assume the vicious attitude. The brace serves as a teacher at each step, but the pupil will find the teacher too severe, if any spasm exists: if the foot is not perfectly flexible and the brace a perfect fit.

To make this brace, a cast of the foot must be taken 
with the foot in the correct position. If made over a cast of the abducted, prone foot it cannot be efficient. Whitman's directions are: "Seat the patient in a chair, in front of him place another chair of equal height; on it lay a thick pad of cotton-batting and cover it with a square of cotton cloth. Put about a quart of cold water in a basin and sprinkle plaster-of-Paris on the surface until it does not readily sink to the bottom, then stir. When the mixture is of the consistency of very thick cream, pour it upon the cloth. The patient's knee is then flexed and the outer side of the foot, previously smeared lightly with vaseline, is allowed to sink into the plaster and, the borders of the cloth being raised, the plaster is pressed against the foot until rather more than half is covered. The foot should be at a right angle to the leg and the whole should be in the plane perpendicular to the seat of the chair. As soon as the plaster is hard its upper surface is coated with vaseline and the remainder of the foot is covered with plaster; the two halves are then removed, smeared lightly with vaseline, and bandaged together. The interior is dampened with soap suds and it is then filled with plaster cream. In most instances it will be of advantage to deepen, in the plaster model, the inner and the outer segments of the arch, in order that the arch of the brace may be slightly exaggerated, especially at the heel, so that the depression of the anterior extremity of the os calcis may be prevented. Upon the model the outlines of the brace are drawn." The brace consists of a sole and two flanges. The sole 
part extends from the center of the heel to the ball of the great toe. While the posterior border lies squarely across the heel the anterior border curves backward and outward from the junction of the middle and internal thirds of the plantar surface at the ball of the great toe to the anterior edge of the external flange. The internal flange is broad rising sufficiently high to cover the astragalo-scaphoid articulation and extending from the posterior to the anterior edge of the sole plate. The external flange, much smaller than the internal, covers the calcaneo-cuboid articulation.

Shaffer's Brace.-Shaffer's method of applying braces is to have a piece of steel cut to fit the shoe, which must itself be a proper fit, with a flange extending up over the inner side of the medio-tarsal joint. This flange is shaped over molds provided for that purpose and of several sizes, designated by their relative heights. The brace is accurately fitted to the patient's foot and any necessary alterations are made before the brace is finished. Completed, it extends from the center of the heel, where it is secured by a single screw, to the heads of the metatarsals. The flange presses outward and slightly upward at the astragalo-scaphoid articulation; the leather of the shoe pressing, or holding, at the two points on the outer side, the heel and the forefoot, so as to produce adduction and inversion. This brace is not absolutely rigid, being secured at the heel only; it is of light weight; it is adjustable and can be readily altered as occasion demands; 
and in a well-fitting shoe, it will retain the foot in the proper position with no discomfort to the patient.

The object of some surgeons in applying a brace in cases of weak-foot, is to obtain proper balance, but it would seem that proper balance can be obtained only with the normally functionating foot; and to treat a foot from the mechanical comparison with skids, piers, arches, et cetera, does not appeal to the physiologist.

Manipulations.-When spasm exists manipulations are clearly indicated. In mild cases the abduction and eversion may be overcome by steady but gentle pressure and the corrected position retained by adhesive plaster strapping. Proper shoes, with the wedge-shaped heels and soles and possibly the extension heel, are ordered and exercises prescribed. The manipulations should be repeated and the strapping reapplied as often as necessary, usually once or twice a week. No braces of any description should be applied so long as the spasm persists. When the foot is perfectly flexible, when the condition has been converted into a simple weak-foot, a brace may be applied if thought to be desirable.

Without Anesthetic.-By using the method illustrated to overcome spasm, all force is applied directly toward correcting the deformity with the least possible pain to the patient. If clumsily performed much force is wasted and the pain is unnecessarily severe. The patient should be seated comfortably, the surgeon standing facing him, at such a distance, that with the leg extended the pa- 
tient's foot will be between the surgeon's knees. The surgeon now grasps the heel with one hand, the left heel with the ieft hand, or the right heel with the right hand, in such a way that the fingers have a firm hold on the

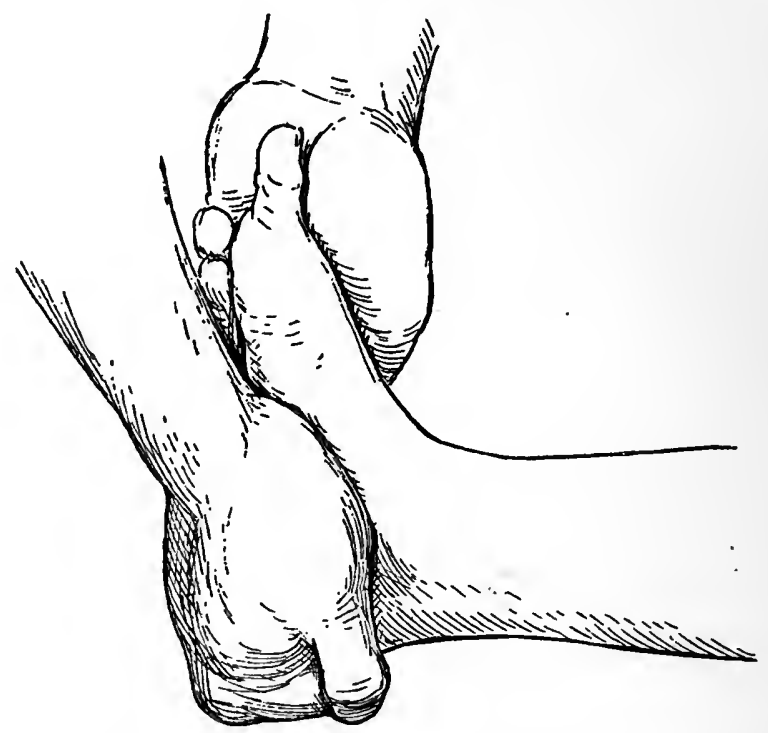

Fig. 36. Forcible Correction of Abduction

One hand so grasps the hind-foot as to firmly fix it and offer the thenar eminence as a fulcrum while the other hand forces the forefoot into the position of adduction. The force used may be supplemented by the physician bringing his knees to bear against the backs of his hands, as recommended by Whitman and described in the text.

outer surface of the os calcis while the base of the palm presses against the inner border of the foot close to the astragalo-scaphoid joint. The forefoot is seized with the other hand, the palm being against the dorsum. The back of the hand which is holding the heel is now brought 
against the knee of the corresponding side of the operator, and the thigh, just above the knee of the opposite leg of the surgeon is used as an added power to the hand

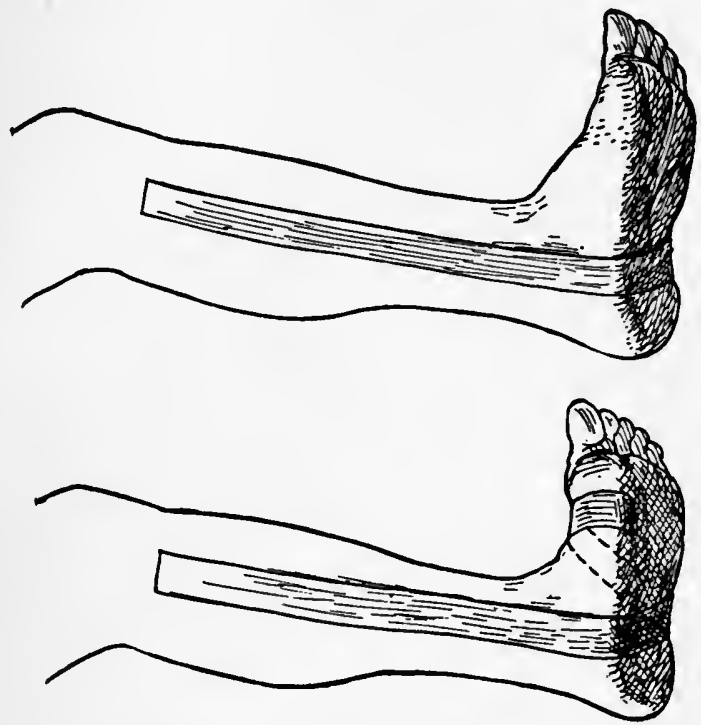

Figs. 37-38. Adhesive Plaster Strapping

Both of the straps are applied and reënforcing straps added as necessary.

Fig. 37 illustrates the strap applied to prevent eversion at the subastragaloid joint.

Fig. 38 shows the strap to hold the fore-foot in adduction and dorsal flexion, which will be accompanied by some inversion. This controls the movements at the medio-tarsal joint and at the ankle joint.

holding the forefoot. Pressure is first made in the direction of plantar flexion; steadily the foot is pressed into the position of equinus to the limit of movement. Then inversion, adduction, and dorsal flexion are performed in 
unison, very slowly and with an even, steady pressure. If the confidence of the patient is lost by a sudden increase of force, which will cause an acute pain, it will be found difficult to obtain perfect correction. If full correction is not obtained, the strapping may be applied and the next séance may be successful, especially if the feet are given complete rest in the interim. Those cases which have too much spasm for this treatment must be given an anesthetic, as will presently be described.

Strapping. - The adhesive plaster is applied to retain the position of inversion, adduction, and dorsal flexion. The inversion may be retained by a strap two inches wide, extending from a little above the external malleolus, around the bottom of the foot and up the inner side of the leg to the internal tuberosity of the tibia. The adduction and dorsal flexion may be secured by another strap encircling the forefoot, just in front of the medio-tarsal joint, and coming from the outer border of the foot across the plantar surface in a diagonal direction inward and backward, and then extending up the inner side of the leg. These straps may be reinforced if necessary. A cotton bandage applied to the entire foot and leg will secure their adhesion. If the removal of the strapping is very painful, on account of the pulling of the hairs of the leg, benzine or some other hydro-carbon, may be applied with a piece of cotton, and afterward the skin may be wiped off with the same material. When perfect flexibility has been obtained, the strapping may be discontinued, but a cure has by no means been accomplished 
until the patient not only can, but does, use the foot at all times in the physiological way.

Severe Cases. - When the spasm is too severe to be overcome with gentle means, when the foot is practically rigid, an anesthetic should be given, the foot forced into

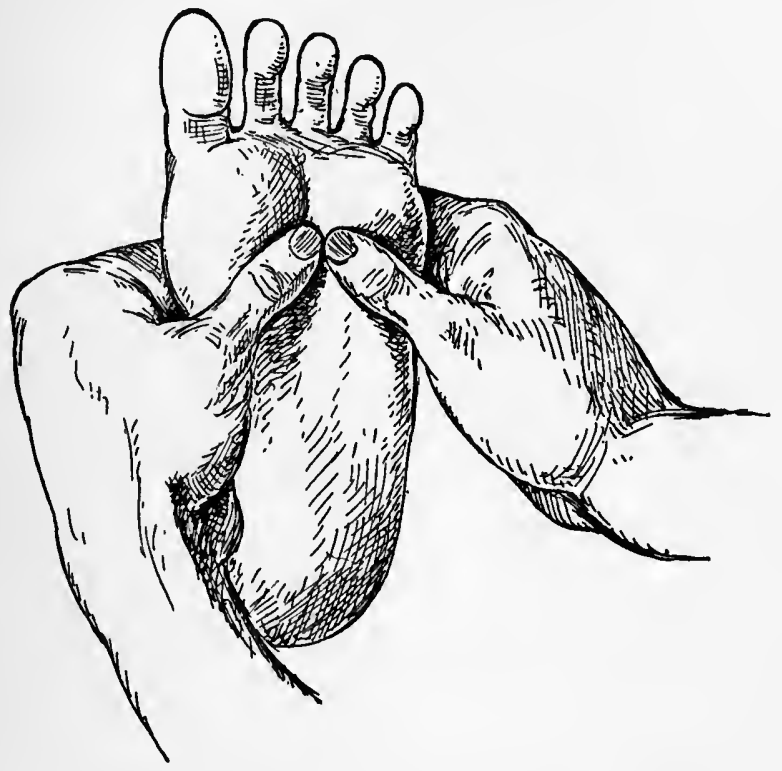

Fig. 39. Manipulation of Metatarsal Joints

an over-corrected, not merely corrected, position, and put in plaster-of-Paris from the toes to the knee. After a day or so of resting the feet in an elevated position, not necessarily in bed, with the object of reducing the swelling and discomfort from the traumatism of the operation, the patient should be encouraged to stand and walk about. 
The more he uses the feet while they are in this position the better will be the result. After four weeks, the dressing is cut down and, if the spasm has entirely disappeared, the case is treated as one of simple weak-foot. Should, however, any spasm remain, the foot must again be put up in a position of over-correction in a plaster-of-Paris dressing.

If any bony changes have taken place, which never-

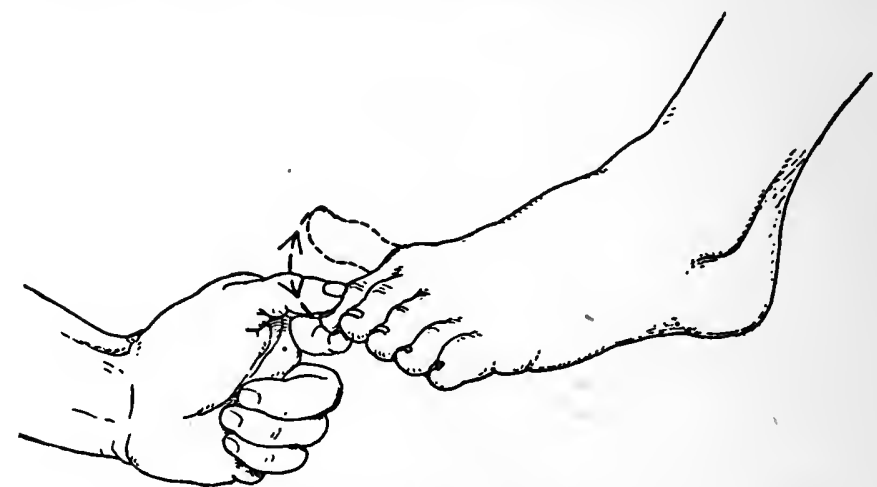

Fig. 40. Manipulation of the Toe-Joints

The rigidity of the smaller joints, especially the toe-joints, should be relieved while the patient is under an anesthetic for mobilization of the foot.

theless permit of restoration of the dome under the anesthetic, the treatment with plaster-of-Paris and the use of the foot in the over-corrected position will have to be prolonged beyond the time necessary to correct deformities due to the muscles and ligaments alone. During manipulation under an anesthetic, attention should be given to all the joints of the foot: the anterior arch should be restored and full flexion (of all the toes) obtained. 
Plaster-of-Paris Dressing. - The object of applying plaster-of-Paris after manipulation of the foot for spasmodic flat-foot, is not only to retain the foot in the improved position, but to cure a more or less severely contused and sprained foot as well. Therefore perfect immobilization must be had, while at the same time provision is made for the swelling which must follow during the first twenty-four hours.

Long strips of cotton-wadding are placed between the toes. They extend from the instep, through the clefts of the toes, to the middle of the sole. They should be of sufficient size to keep the toes slightly separated, thus giving a broader base to the forefoot and preventing the toes from being crumpled up and also keeping adjoining skin surfaces apart. Cotton-wadding is then applied from the tip of the great toe to the tubercle of the tibia. The easiest way to apply this, is to have the wadding cut into strips three inches wide and rolled on itself the same as a bandage. The thickness to which this is to be applied must depend upon the amount of swelling which may be expected. Usually it should be about half an inch thick over the entire part, with an extra thickness of half an inch over the instep and about the malleoli. Over this wadding a flannel bandage may be applied for the purpose of retaining the wadding in place while the plaster bandages are being put on. This flannel bandage is for no other purpose; and care must be exercised that it is not made to bind at any part. Many surgeons teach that a plaster dressing should begin 
at the metatarsal-phalangeal joints, but if the entire toes are included there will be much less likelihood of serious disturbance with the circulation.

To hold the foot in the over-corrected position, not

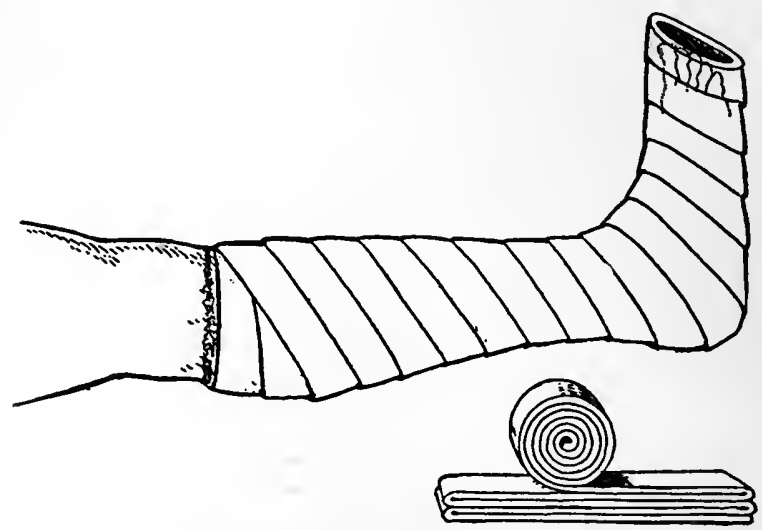

Figs 4I-42. The Plaster-of-Paris Bandage

It should extend from the tips of the toes to the tibial tubercle. It should be laid on and not bound on. Spiral turns are less likely to offer ridges than are figure-of-eight turns. Antero-posterior compression of the completed plaster over the forefoot assures plenty of room for the toes. Lateral compression above the heel will bulge the plaster out backward and relieve the tendo Achillis from all danger from pressure.

Fig. 42 illustrates the making of a pad to be incorporated into the plaster over the bottom of the foot and provide a sole. It may be easily built up on one side or the other or under the toes or under the heel so as to act as a corrective force when the plaster is used as an ambulatory splint.

a little skill and dexterity are needed. From the beginning of the application of the plaster bandages, until the dressing is well set, the foot should be held continuously in the desired attitude. If, after a few layers of the bandage have been put on, the position of the foot 
is altered, wrinkles will be formed, which may cause pressure sores. The assistant should insert the fingers of one hand between the dressing and the skin, grasp the toes firmly, and hold the foot in the dorsal flexed, adducted, and inverted position. The other hand is used to press against the inner side of the leg so as to help in obtaining the inversion and adduction. If a good grip is obtained of the toes, that hand will not need to be removed until the dressing is finished.

The plaster-of-Paris bandages should be two or three inches wide. They are placed, one at a time, in warm water until thoroughly soaked, and as one is being used another is put into the water. In applying the bandage care must be taken that it is not bound on with any firmness: each layer is "laid" on, as the bandage is unrolled upon the foot and leg. It should not be held in the hand as when a bandage is used in applying splints or in securing the dressing after an open operation, but allowed to rest on the part and to be rolled about the foot in such a way that, as it unrolls, the layers may be left where they fall without any undue pressure. Should any part present a wrinkle it must be straightened out so that the bandage lies flat in every instance.

A most important part in the technique is the thorough rubbing of each layer. If an assistant does nothing but rub the layers of bandage as they are applied, until the plaster is perfectly set, it will be found that a much greater hardness is produced than if this is neglected. Before the last layer is applied about the toes and at 
the upper end, the wadding is turned over the plaster and the last layer of bandage is used to secure the turned over part. This will pad these edges which might otherwise cause excoriations. Finally, talcum powder may be rubbed into the entire dressing, filling any pores in the last layer of bandage and giving a smooth finish which will permit washing of the bandage should it become soiled. Before the plaster has become hardened, the forefoot about the metatarsals, should be compressed in the vertical diameter, flattening it and making more room for the toes.

Operations.-For the cure of these conditions of the foot, operations are seldom necessary. Manipulations, patiently, thoroughly, and repeatedly carried out, will, in the great majority of instances, effect a cure.

Tenotomy.-The operation which is the most frequently called for, is the simple one of tenotomy. The peroneal tendons are the ones which most frequently need to be divided. It is well to be prepared to do this whenever a flat-foot is to be manipulated under an anesthetic. After painting the skin about the external malleolus with iodine, a small tenatome is inserted two inches above the tip of the malleolus close to the posterior border of the bone and both tendons divided subcutaneously. Division of the tendo Achillis should be done whenever manipulations fail to restore the normal range of dorsal flexion. As the foot is to be treated in plaster-of-Paris subsequently no hesitancy need be felt in doing these or other tenotomies if the sought- 
for over-correction can be the better secured by so doing, as the immobilization will assure one of perfect union.

Excision.- Excision of the head of the astragalus: "This is the best operation on the whole," says Cheyne, but according to this surgeon it may leave a weak dome, necessitating the continual use of a support.

Arthrodesis.-Ogston's operation: an arthrodesis of the astragalo-scaphoid joint. The incision is made from just below the internal malleolus, forward to just below the tubercle of the scaphoid, avoiding the tendon . of the tibialis anticus. The cartilage on both articular surfaces is removed and also any bone necessary for the restoration of the dome. A peg of ivory or of other material is inserted through a drilled hole, passing from the scaphoid into the head of the astragalus. The foot is kept in a plaster-of-Paris dressing for four weeks and a support is worn for six weeks longer, or until ankylosis is firmly established.

Tarsectomy.- Internal tarsectomy is done to shorten the internal border of the foot. By removing a wedge-shaped piece of the bony formation of the foot, -regardless of just what bones are attacked,-with the base of the wedge on the inner side and facing in a direction inwards and upwards rather than directly inwards, and the apex outward and downward, reaching quite to the external border, one may restore the adduction and the inversion. The writer has had no experience with this operation, does not believe it is often necessary, does not find it very popular among his con- 
frères and would use it only after every other effort had failed in a foot which was practically useless on account of pain from the deformed position.

Scaphoidectomy.-This was done by Golding-Bird in four cases, in two of which the head of the astragalus was also removed. All were relieved of the pain from the removal of pressure on the outer side, and in one case the arch was restored: in this one there was added a subcutaneous sawing-through of the whole tarsus, thus admitting of rotation and adduction of the front on the back part of the foot.

Post-Operative Treatment.- Post-operative conditions, even after nothing more than a manipulation under an anesthetic, may be a source of delaying a cure. Adhesions may form which cause great pain upon attempts at the full range of movements.

Douching.-The easiest remedy for this condition and at the same time the best, is douching alternately with cold and hot water. The nervous shock to the dilators and contractors of the vessels, creates a splendid local circulation; and the force of the water, if the douching is rightly done, is of an added value. The hot water should be as hot and the cold as cold as the patient can bear. Two pitchers may be used, each holding half a gallon, or else smaller pitchers, refilled as often as necessary, until about a half-gallon of each water is used. The foot is placed over a tub of some sort to catch the water. With one of the pitchers held eight to twelve inches above the foot, the water is poured onto it in 
a small stream for thirty to fifty seconds, the time being regulated by the sensations of the patient: the foot should feel quite hot when the hot water is changed to the cold and quite cold when the cold water is changed to the hot. The pitcher may be held as high as 20 or 24 inches above the foot if desirable, and this is usually advisable after the first few treatments, as the greater force is very beneficial. It may be possible to use a spray from the tap when the tap has both hot and cold water connections, but this is seldom satisfactory as the hot water usually becomes too hot in a very few moments. When douching is impossible, we find that using two foot-tubs or pails, one containing hot and the other cold water, and having the patient put the foot alternately into one and then into the other, is productive of excellent results. Under no circumstances should hot water alone be used. For tired, aching feet, hot water is soothing but it is not good for the circulation. Cold water, followed by a vigorous towel-rubbing, is always to be preferred when inflammation is absent.

It will be found that immediately after the douching both active and passive movements are much freer. The patient should flex and extend the foot at the ankle, flex and extend the toes, invert and evert the foot and circumduct the forefoot:

Liniments.- Because they provide a source of massage, and are cooling to any inflammatory condition, liniments may be advantageously prescribed. A liniment which is more satisfactory in many ways than 
I IO DISEASES AND DEFORMITIES OF THE FOOT the ordinary ones of the pharmacopœia, is the one recommended by Shaffer :

R.

Chloral hydrate

Menthol

Pulv. gum camphor aa3 III

Olei cajuput $\quad 3 \mathrm{VI}$

Tincturæ belladonnæ $\mathbf{3} \cdot \mathrm{IV}$

Spiriti vini rect. q. s. ad. 3 IV

M. Sig. Liniment. Poison. Use as directed. 


\section{CHAPTER V}

\section{CONGENITAL CLUB-FOOT}

THE fixation of the foot at birth in a position in which the normal foot can be placed, or an exaggeration thereof, is called congenital club-foot. If untreated, in the severer forms, when the child begins to walk, it will walk on the ankle and hence the name, talipes.

Varieties. - If the foot is fixed in the position of plantar flexion, with the movements normal in the vertical; medio-tarsal, and in the longitudinal; sub-astragaloid axes, it is called talipes equinus. If the foot is fixed in the position of dorsal flexion, with the other movements normal, it is talipes calcaneus. If movement at the transverse axis, the ankle joint, is normal and the foot is fixed in the position of inward rotation on the longitudinal axis and adduction on the vertical axis, the condition is named talipes varus; or if the fixation is limited to the same axes but the foot is everted and abducted, talipes valgus.

There may be, however, a combination of one of the first two deformities with one of the latter two, such as talipes equino-varus, talipes equino-valgus, or talipes calcaneo-varus or talipes calcaneo-valgus. There are, in addition, cases in which the dome of the foot is ab- 
normally high or abnormally low, when the condition of cavus or planus exists.

Normally, at birth, the foot is slightly in the position of equino-varus and the arch is not well formed. It does not constitute a pathological condition, when by voluntary or reflex action the muscles can, unassisted, execute the normal movements.

A classification of the deformities of club-foot based upon the nerve supply to the muscles, was arranged by Bonnet, and in spite of its imperfections, it is very useful. Malgaigne, as quoted by Lannelongue, criticised it because the tibialis anticus, supplied from the external popliteal, elevates the inner border and valgus could not accompany its contraction; and also because in Bonnet's tabulation of the degrees of club-foot he places calcaneus as the last or fifth under external popliteal club-foot, not admitting therefore that it exists as a simple variety. This classification is:

Internal Popliteal Club-foot.

I. Elevation of the heel.

2. Antero-posterior flexion of the foot on itself.

3. Abduction of the fore-foot.

4. Turning of the heel inward.

5. Increase of the dome of the foot.

External Popliteal Club-foot.

5. Lowering of the heel.

4. Forced extension of the foot on itself.

3. Adduction of the fore-foot. 
2. Turning of the heel outward.

I. Lowering of the dome of the foot.

Frequency.-The frequency of congenital clubfoot is one in every two thousand births (Lannelongue) to one in every eleven hundred (Bessel-Hagen). In comparison with other deformities it bears the relation of one to five. Tamplin reported that at the Royal Orthopedic Hospital in 10,2I7 deformities of all kinds, I,780 were congenital club-foot. Kirmisson found it a little less frequently, I66 cases out of I, I00 deformities at the Enfants Assistés; and Bessel-Hagen placed the frequency at one in ten. Lechiberder, quoted by Lannelongue, in 15,229 births found the following abnormalities; club-foot 8 , spina bifida 4 , hare-lip 8 , umbilical hernia 77 , hydrocephalus 3 , anencephalus 2 , imperferate anus I, hypospadias I2, extrophie of the bladder $\mathrm{I}$, and encephalocile 2.

It is very unusual to see different forms of the deformity in the same patient. Whitman reports eighteen cases, less than 3 per cent.; Ketch three cases, slightly more than 3 per cent.; and Kirmisson about the same percentage.

It is more often double than single, when unilateral the right is more often affected than the left (Whitman, Ketch, and Kirmisson); but Bessel-Hagen found fiftyone left to forty-one right.

Boys are more frequently affected than girls.

Of the varieties, equino-varus is by far the most com- 
mon, three-quarters of all cases (Whitman). Pure valgus and pure equinus are rare, according to Walsham and Hughes, Kirmisson, and others. The existence of pure congenital equinus is denied by some. It was seen but twice in twelve years by Walsham. Whitman reports 40 cases of equinus and 123 cases of valgus out of $\mathrm{I}, 660$ cases.

Etiology.-The etiology of congenital club-foot has received the careful study of many eminent physicians. Scarpa, Little, Lannelongue, Volkman, Hoffa, Berg, J. Guerin, Parker, Walsham, and Scudder have perhaps thrown more light upon the matter than any other students. They by no means reached the same conclusions and no one has as yet succeeded in advancing a theory, regarding the cause of this deformity, which encompasses all cases, meets the laws of embryology, and explains satisfactorily the pathological changes. These various theories, - and they are all theories,-are usually termed the germ theory, the nerve theory, the bone theory, the pressure theory, and the theory of abnormal fetal movements.

Although, in a few instances, this deformity has been traced through several generations, its appearance is generally an isolated case in the family and all, I believe, are agreed that heredity can not be a factor. Consanguinary marriages have been cited as of possible etiological significance, but any connection is only theoretical. Broadhurst believes that impressions and emotions dur- 
ing pregnancy may cause club-foot; but few, I believe, will agree with this opinion.

The germ theory is satisfactory where there is evidence of positive germ effect, as absence of bones; but it is not acceptable in the vast majority of cases.

The nerve theory, that some nerve lesion, perhaps transitory and functional only, has caused the deformity in the same way as spastic paralysis may cause the deformity post-natal, had for its principal supporters, Little, Adams, and J. Guerin. According to this theory the muscles hold the feet in the deformed position while the changes in the structures follow in adaptation to the new position.

The bone theory attributes the deformity to a primary abnormality in the bones, chiefly the astragalus, which retains the foot in the deformed position; and the soft structures accommodate themselves to this position. As will be seen when we come to study that bone, the fetal astragalus has the direction of its neck very similar to that of the talipedic astragalus, more inclined downward and inward than the normal astragalus at birth. However, the talipedic astragalus shows by its scaphoidal articular facet that there was some other influence at work to alter the relations between the tarsal bones.

The pressure theory holds that the foot was held by pressure, uterine walls, twins, tumors, abnormalities such as hydrocephalus, adhesions of the amnion, etc., in a deformed position for such a length of time that 
the alterations in bone, ligaments, and muscles are permitted to follow. Hippocrates, Galen, Scarpa, Pare, Volkman, Hoffa, Shattock, and Parker are among the supporters of this theory. Its opponents claim that there are other parts which are retained during fetal life for some time in one position without the creation of deformities such as flexed neck, arms, etc., and furthermore that pressure could scarcely produce the extreme elevation of the os calcis which is present in severe cases. Pressure sores have been discovered on fetuses and new-born children, but they are rare and can not be considered of much value in confirmation of this theory.

The theory of abnormality in fetal movements, as advocated by Eschricht, Berg, and Kocker, is that instead of the positions of the lower extremities being altered in utero, especially during the third and fourth months, the one position is continued for such a long time that shortening of the muscles and ligaments results, together with changes in the bones. The deformity results, in both this and the pressure theory, from too long retention in one position.

General Appearance.-In congenital talipes equinovarus, if only one foot is affected, its external appearance may easily be compared with the normal one: it is smaller; its color proclaims a poorer circulation; its toes are pointed downward and inward toward the other foot; its sole is turned inward, upward, and backward and the dorsum faces forward, outward, and downward; the ex- 
ternal border is much rounded and the internal is markedly concave; there may be an angle interrupting this concavity at about its center; the external malleolus is more prominent and the internal may not be apparent; the tuberosity of the os calcis is smaller, elevated, and near to or quite touching the external malleolus. On the dorsum, there may be seen and easily felt, the astragalus protruding from its mortise. The internal malleolus may be felt to be apparently in advance of its normal position and the external will seem to have taken a position nearer the back of the leg, due to the inward deflection of the astragalus and the foot as a whole. Close to the internal malleolus, sometimes beneath it, may be felt the tuberosity of the scaphoid. The head of the astragalus will be slightly above and behind the scaphoid tubercle.

Explanation of Appearance.- What has taken place may be the more easily understood by keeping in mind the three principal axes of the foot, and imagining each movement about these axes as being strongly accentuated. The movement on the transverse axis, in the direction of plantar flexion, is so exaggerated that the trochlear surface of the astragalus is protruded for perhaps two-thirds of its length, forwards, out of its socket. Attention has been called to the direction this bone takes in plantar flexions; not only about a transverse axis but also about a vertical axis which is situated at about the internal malleolar facet, an inward rotation, and consequently we find the head directed inward as 
well as downward, instead of simply forward and downward as it would, were the movement limited to an antero-posterior plane. On the antero-posterior axis, passing through the sub-astragaloid joint, the foot has been strongly inverted so that the entire plantar surface is directed inward. There is also some movement in this joint about a vertical axis and in this condition this movement, normally not marked, is exaggerated, so that there is a twisting inward of the anterior part of the os calcis and outward of its posterior tuberosity. Thus an alteration is produced in the normal relation of the long axis of the os calcis with that of the astragalus. Through the vertical axis, at the medio-tarsal joint, adduction of the forefoot has taken place in the normal direction of the movement here but unusually pronounced. At this joint too, the usually but slightly marked movement of rotation, will be marked in an inward and upward direction.

Changes in the Bones. - The most marked changes in the bones will be found in the astragalus and the os calcis: the astragalar neck will be directed inward and downward instead of forward and very slightly inward and downward. In the fetus it is normally turned inward to about 35.75 degrees, in the adult to about $\mathbf{1 2 . 3 2}$ and in the club-foot to about 50.75 (Scudder). The astragalar head is much attenuated and presents an extension backward of its articular surface. There may be a ridge across the articular surface separating the posterior part, which is in articulation with the scaphoid, 
from the anterior part, which, in the deformed position, is left uncovered by the scaphoid.

The os calcis is not only plantar flexed and rotated on its long axis and turned on a vertical axis, so that its anterior extremity is inside of its normal position, as already pointed out, but there is an inward twist, postero-anteriorly, of the bone: an intrinsic twist. The superior surface of the os calcis looks forward and inward. The tuberosity is small and may be in contact with the external malleolus. The facet for articulation with the cuboid looks markedly downward and inward. All the bones of the foot are undeveloped, otherwise the rest are nearly normal in their characteristics.

The abduction and rotation of the forefoot at the medio-tarsal joint carries the scaphoid inward, backward, and upward, so that it articulates with the back part of the astragalar head and may touch the internal malleolus.

The cuboid is likewise altered in its relations to the os calcis; its superior surface looks outward and downward. The malleoli are not much altered in development. There is no change in their relative position to each other, although the position of the foot gives the appearance of the shifting of the external malleolus to a more posterior position. There may be an articular facet on the external malleolus for the os calcis and one on the internal malleolus for the scaphoid.

The bones of the leg may be twisted in their long axis, so that were the foot normal it would nevertheless 
be turned inward in its relation to the knee. The femur may be twisted inward on its long axis and the femoral neck may be so bent that it is slightly concave forward and adds to the inward twist of the shaft of the bone.

Changes in Ligaments and Muscles.-The ligaments on the inner side of the ankle and foot and the inner half of the plantar ligaments and the posterior ligaments, are all more or less shortened. The muscletendons passing over the ankle-joint are displaced to an extent depending on the changed position of their points of insertion. The tendon of the tibialis anticus will lie close to the internal malleolus, either in front of it or, in severe cases, to its inner side, as the internal cuneiform and the first metatarsal are in that direction. The tendons of the other anterior muscles will be likewise displaced inwards to a corresponding degree.

The tibialis posticus, instead of turning forward beneath the malleolus, continues downward to the tubercle of the scaphoid; and as the scaphoid approximates and may even articulate with the malleolus, the portion of this tendon below the malleolus may be very short and difficult to define. The tendon of the flexor longus digitorum and the flexor longus pollicis are displaced inward from their normal position in proportion to the degree of the deformity. The peroneal tendons have deeper grooves than usual on the external surface of the os calcis. The longus, instead of crossing the cuboid in its course to its insertion, may pass posterior to the cuboid. The tendo Achillis is displaced outward with 


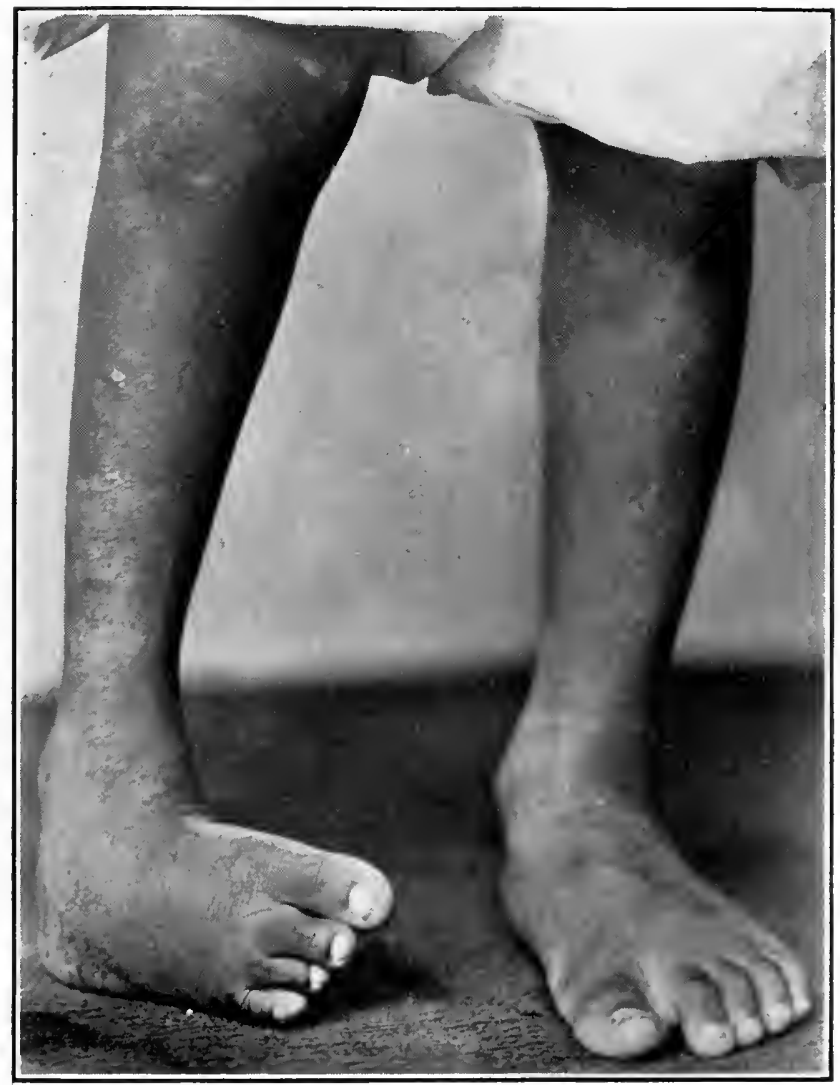

Plate II.-Unilateral Coxgexital Talipes Equino-Varus

The followlng characteristles are illustrated: the atrophy of the leg and foot; the elevated position of the malleoli due to the equinus; the callus over the head of the os calcis where most of the weight is borne: the rolling up of the forefoot on a longitudinal axis, plantar-ward, the little toe belug out of sight and the fourth turned inward and upward. This foot was cured by multiple tenotomies and resection of the head of the os calcls, followed by a brace. 

the tuberosity of the os calcis and lies closer to the fibula than normally.

The blood vessels and nerves are shortened, according to some observers, while others believe that the vessels are not actually shortened but are tortuous.

Pathology After Use.- If such a foot as has been described is walked on, all the deformities become more
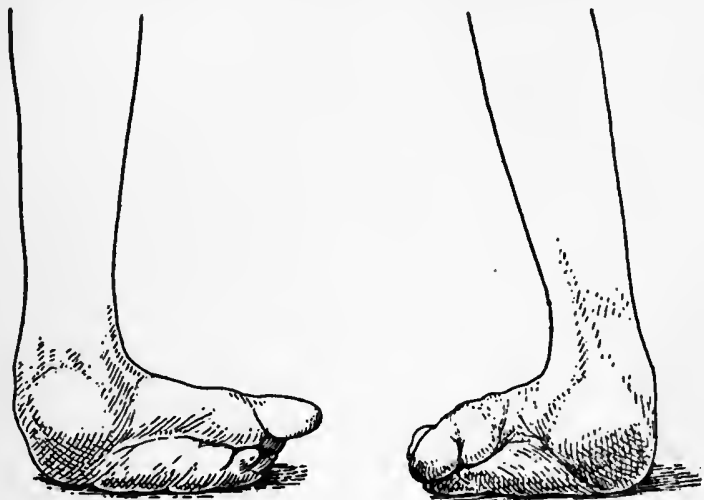

Fig. 43. Congenital Talipes Equino-Varus

Illustrating a congenital club-foot which has been walked upon. Note the longitudinal crease in the plantar surface and, less marked, the transverse crease in front of the heel.

pronounced; the equinus, the inversion, and the adduction. The dorsum may eventually come in contact with the ground. Usually, however, it is the anterior and external part of the os calcis which receives the most weight, and a callus, with a bursa beneath it, is generally found in this location.

The plantar surface, facing inward, upward, and backward, will become narrower from pressure. This pres- 
sure tends to roll the foot upon itself and, in consequence, a longitudinal furrow will be found in the plantar surface of a congenital club-foot which has been walked on. Just in front of the heel, at the medio-tarsal joint, a transverse groove may be seen which will be most marked between the posterior part of the longitudinal groove and the internal malleolus.

Through use, the os calcis will be raised still higher, so that the bone may assume a position parallel to the fibula and may even articulate with it, a capsule sometimes completing the joint. Its posterior superior articular facet may be altered in the direction of its long diameter so as to be directed from behind, forward instead of forward and slightly outward, or, in severe cases, it may be directed forward and markedly inward. Attention is called to these changes by Walsham and Hughes, and interpreted by them as resulting from an altered part of the bone being brought into the articulation and not from a shifting of the direction of the normal articular surfaces. The anterior surface of the os calcis is much altered. The cuboid in a used talipedic foot of this variety is still further displaced internally and the position of the articular surface on the os calcis, for the cuboid, has not only an absence of articular cartilage but presents an over-growth of bone, a tuberosity. This has probably been produced by irritation, as it is here that the greatest support is obtained in walking and standing. This tuberosity is of great importance in attempting to replace the cuboid in reducing de- 
formity. The internal surface of the os calcis presents a new articulation for the cuboid. This surface has its concavity increased and the external surface, its convexity increased.

The astragalus will have lost the articular cartilage from the anterior protruding part of the trochlear. The posterior part of the trochlear will be separated from the inferior part of the bone only by a ridge. The exposed inner portion of the head, the anterior portion of the talipedic head, will have lost its cartilage. The neck will have a decided downward as well as backward inclination and will, according to Walsham and Hughes, be elongated. On the external surface, in front of the external malleolus, there will be found a bony deposit which would interfere with the outer rotation of the bone into its normal position.

The other bones will show some changes, but none that are of equal importance to those of the os calcis and astragalus.

All the above changes will be found in the congenital talipedic foot of the equino-varus variety, which has been used, varying in the degree of their pronouncement with the degree of deformity at birth and with the amount the foot has been employed.

Confirmation of Structural Changes.- Whereas the changes in the muscles, ligaments, and bones during infancy are such, usually, as can be overcome with comparative ease, in childhood and adult life they become more and more serious obstacles to a cure. Slight 
124 DISEASES AND DEFORMITIES OF THE FOOT

manual traction may at the beginning be sufficient to restore the normal position; but as months are allowed to pass without treatment, tenotomies become more and more necessary. Further neglect may result in the impossibility of a cure without the division of ligaments, of part of the plantar fascia; and even without some operation on the bones. 


\section{CHAPTER VI}

\section{TREATMENT OF CONGENITAL CLUB-FOOT (TALIPES EQUINO-VARUS)}

No time should be lost in beginning treatment. Some emphasize this rule by insisting that treatment be begun the day of birth, "at the hour of birth" (Willard). Others postpone giving any attention to the deformity until the baby is two weeks old. Delay, however, of several months is simply neglect.

\section{EARLY TREATMENT}

Early treatment consists of frequent gentle manual correction and the employment of some means of retaining the foot in an improved position. Frequently this improved position will become in a few weeks the fully corrected or even an over-corrected position. The degree of the deformity and the resistance met in overcoming it, should determine the method to be followed. Cases which, from the beginning, show a marked improvement from manipulation and simple bandaging may be thus treated as long as improvement continues. As soon, however, as improvement becomes arrested, greater care must be given to the retention apparatus. The foot must be held at all times in the best possible position; 
only in this way can progress be made. If the apparatus used is a removable one and if massage and movements at the joints are practiced, care must be exercised to prevent the assuming of the deformed position, even for a moment, while the apparatus is off. The apparatus should not be removed from both feet at the same time: one only is removed, that foot massaged and exercised and the apparatus re-applied, and then the same treatment is given to the other foot.

Manipulation._- "Of all curative measures, manipulation stands first" (Willard). Bearing in mind the three axes of the foot through which the deformity occurs, gentle momentary attempts are made to turn the foot on these axes toward the normal position. The leg is held firmly about the ankle with one hand, the right leg with the right hand; and the forefoot grasped with the other hand in such a way that the plantar surface rests in the palm of the hand, the fingers encircling the outer border, the thumb the inner; and movements are made to overcome the adduction and inversion. By holding the first and second fingers of the hand holding the leg,- - the right hand if the right leg is being manipulated,- against the outer surface of the os calcis, the surgeon can concentrate his force in attacking the adduction. If the os calcis is not held, the force will be exerted toward correcting both the adduction and the inversion. By placing the palm of the hand, the right in treating the right foot, against the plantar surface in such a way that the heel rests in and is grasped by the 
fingers, the toes resting against the base of the palm, he may direct all his force to obtaining movement through the antero-posterior axis, overcoming inversion, and also through the transverse axis at the ankle, overcoming equinus. The deformity of equinus, however, demands little attention until the others, the adduction and the in-

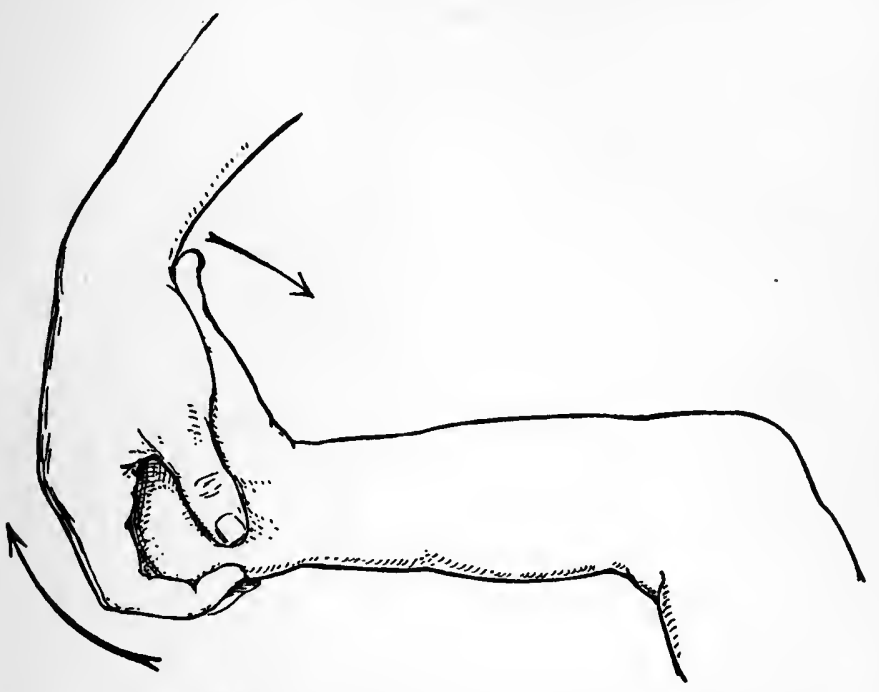

Fig. 44. Manipulation of Congenital Club-Foot Position of the hand and the direction of the force in overcoming inversion and equinus.

version, have been practically cured. There are two reasons for this: the tight heel-cord gives an excellent fulcrum against which the force used in correcting the other deformities may be exerted and without which it is difficult to obtain a fulcrum; and the equinus seldom offers much difficulty of itself, even if neglected for some 
months. Furthermore, when walking is begun, the weight of the body usually does much to correct the equinus, and should this not be all that is necessary, division of the tendo Achillis is a simple and satisfactory procedure.

The manipulative treatment should not consist of a séance of yelling and screaming. It is not necessary and it far from increases the confidence of the mother. The object is not to accomplish much in any one treatment, only by frequent treatments. Manipulation is too often practiced as though it had some occult power and is sometimes prescribed in a more or less careless way. The administration of manipulation is by no means an indifferent matter. The regularity of treatments, the time given to each treatment, and the detail of each treatment are all of vital importance if one would meet with success. If manipulation is to form an important part of treatment, and it should in every case, it is absolutely necessary to take the time and to exert the patience to teach some member of the household how to do it. It seems to me that the success of the teaching depends more upon the physician than upon the intelligence of the mother or nurse, who can generally be counted on to carry out faithfully the instructions as taught. The exact hours of the day that the manipulations are to be performed should be prescribed as carefully as are the hours for nursing an infant or the administration of a medicine. The number of times that each manipulation is to be done should be designated; as twenty of these move- 
ments, twenty of these others, and ten of these. The massage should be prescribed by time; as rubbing the leg for one minute and the foot for one minute, by the clock. Then the re-application of the apparatus must be taught in the minutest detail. The laity are now so accustomed to being taught details, as for instance, regarding the temperature of the bath, the preparing of the bottle, etc., that the statement that they are unable to follow instructions as to manipulations of a deformed foot or of the application of a brace, is, it might seem, a reflection upon the physician as an instructor.

For the first few weeks the manipulations should be done by the physician as frequently as possible, if not daily, at least several times a week. Subsequently the physician need see the patient but once a week, and, if all is going well, the time may be increased to two weeks. The resistance which the deformity shows and the care with which his instructions are carried out, must be the determining factors in deciding on the frequency of the visits. At each visit the mother or nurse should go through with the treatment exactly as she is accustomed to do it at home. The physician is thus able to correct any little mistakes. Otherwise errors in treatment or in the application of the brace are likely to creep into the procedures. If there comes a halt in the progress of the improvement, then some change must be made in treatment. The manipulations are not being performed regularly and exactly, or the brace is not doing the work for which it was designed. If the fault can not be located 
and remedied, the brace had better be discarded and plaster-of-Paris substituted. Some surgeons are unable to fit a brace so that it will be efficient, and if they are adepts at applying a plaster-of-Paris bandage they had much better not attempt the use of a brace at all.

Cases come to us all which are more fitted for treatment with plaster than with a brace, and it therefore should be within our range of skill to use whatever a case may demand, just as we must be ready to operate when an operation is necessary.

Apparatus.-Muslin Bandage. In some few cases a simple muslin bandage may be used. It should be one and one-half inches wide for an infant and about three yards long. This is applied with a few turns about the foot, close to the toes, and is then extended up the leg. It should be applied so as to unroll the foot; beginning at the outer border of the foot, it should pass over the dorsum to the inner border and thence across the plantar surface, these turns being repeated three times; then the bandage should be carried up the outer side of the leg and traction made, the foot being pulled into a position as nearly approaching valgus as may be, and the position so held with one hand while with the other the bandage is secured by a few turns about the leg and then continued down to the toes in a spiral fashion. Though this bandage may be of some service as a temporary measure while a brace is being constructed, not much can be expected from it. The forefoot slips around in the turns about it and even if much force is at first 
obtained toward correcting the varus, it will soon be lost by the slipping of the bandage down the leg.

Adhesive Plaster.- A strip of adhesive plaster applied in the same way will be much more effective. After a few turns about the forefoot with a strip three-fourths of an inch wide, it is carried up the outer side of the leg and, while the foot is held in the best position attainable, it is made to adhere to the upper third and is further secured by a strip encircling the calf. If a fairly good position has been obtained, the lower third or so of the piece extending up the leg will stand away from the skin. A cotton bandage is applied from the toes to the knee. With this adhesive plaster strapping some manipulations may be performed without its removal and the muscles and the skin other than that covered by the plaster may be massaged and kept clean and healthy. After the plaster is applied to the forefoot and before the leg strip is secured, care must be taken that with the foot held over in valgus the edge of the plaster does not cut into the skin, as may easily happen. As with older children and with adults, some babies' skin is easily irritated by the plaster and it will not be tolerated. In these cases a cotton bandage must be first applied and the adhesive outside of that; but this method is not at all satisfactory as the plaster is robbed of much of its usefulness.

Braces.- Perhaps the simplest brace is the one devised by Judson. It consists of a piece of brass or other metal, about four inches long and one-half an inch wide, with cross pieces riveted at either end. These cross 
pieces are very light and easily molded with the hand to any desirable shape. One cross piece is to fit about the calf of the leg and is about two inches long and three-

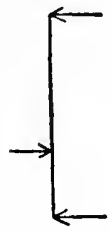

Fig. 45

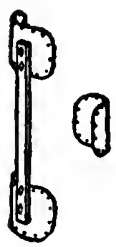

Fig. 49

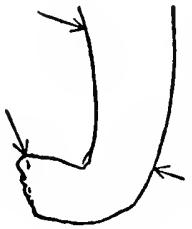

Fig. 46

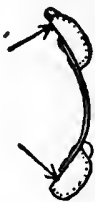

Fig. 47

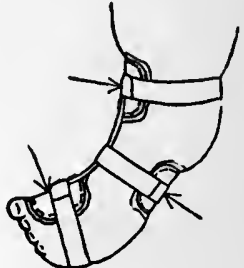

Fig. 48

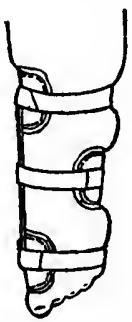

Fig. 50

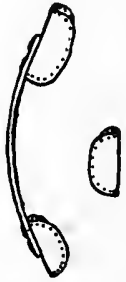

Fig. 5I

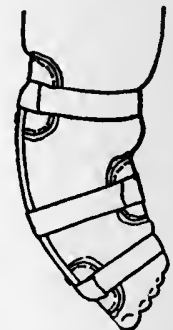

Fig. 52

The Judson Club-Foot Brace

In Fig. 45 the arrows indicate the directions in which pressure is to be obtained and the points of application of these forces to the deformed foot are shown in Fig. 46.

Fig. 47 illustrates the brace and Fig. 48 its application.

As improvement is obtained in "straightening the deformity, the brace is straightened as in Fig. 49 and applied as in Fig. 50.

Finally the brace is bent into the opposite curve to that in which it was first applied, Fig. 5I, and when secured to the foot, Fig. 52, produces an over-correction.

quarters of an inch wide. The other is to fit about the forefoot and is one and a half inches long and one-half an inch wide. The length of the calf-band and the footband should be such as to encircle two-thirds of the calf 
and foot respectively, and therefore the above measurements are only approximate. Each is riveted by one rivet so that it may be turned to lay flat against the skin, regardless of the direction of the long bar, and may not cut into the tissues. The completed apparatus is covered with chamois or other soft material. The long bar is now curved with wrenches so that it will lie flat along the leg from the inner side of the calf to the inner border of the foot at about the center of the first metatarsal, with the foot in its best possible position. On account of the equinus deformity, this will be about a straight line. That portion opposite the ankle and the mediotarsal joint is then bent so as to lie about one-half an inch away from the ankle and foot. Small strips of adhesive plaster are made to complete the circumference of the bands about the calf and forefoot, and a third strip draws the ankle close to the upright bar.

This apparatus is easily removable and applicable, and is so simple in its construction that it can readily be made by any surgeon. The material for making it can be found in any village or on any farm. Any piece of tin used in several thicknesses to give strength and riveted together will answer the purpose; and any soft cloth may be used to cover the brace. Two monkey wrenches screwed down to grasp the long bar on the flat, will give ready means for shaping the brace as desired.

Felt Splint.- Poroplastic felt may be shaped into a retention splint and acts very well if properly constructed. However, it can not be altered to fit improved positions, 
is not always readily obtainable, and requires more experience in making than do most braces. It usually needs some reënforcement, and great care is demanded so that creases on its inner side do not cause excoriations. To make it: For a pattern, take a piece of paper long enough to reach from the calf at a point opposite the tibial tubercle to the end of the great toe and wide enough

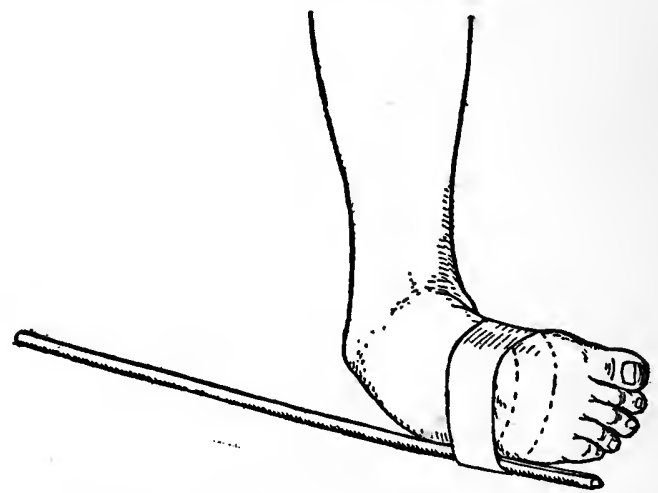

Fig. 53. The Single Steel Bar Brace

This brace is suitable for use in early cases. The deformity of adduction is the one which yields the most readily to its application. The bar, properly padded, is secured to the outer border of the foot.

to reach half way around the leg and foot. A wedgeshaped piece is cut out of each side opposite the ankle, and any trimming necessary is done. The pattern is then laid upon a piece of felt and the felt cut to conform with it. The felt may be softened in a number of ways; - by placing it in hot water, $125 \mathrm{~F}$., until it is soft, when it is rapidly dried between towels and applied and molded to the leg and foot,- the foot, of course, 
being held in the best possible position,- and a cotton bandage smoothly and firmly applied. In a few minutes the felt will be hardened and it is then removed and

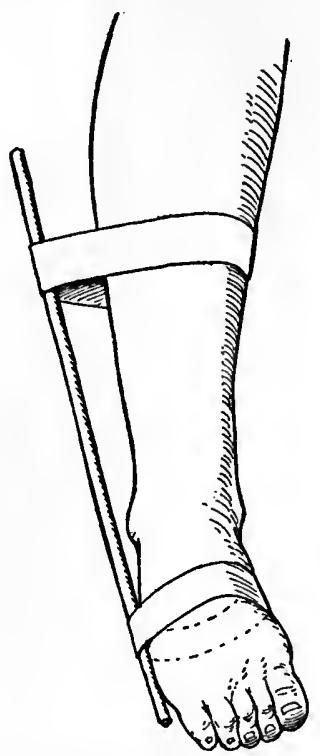

Fig. 54. The Single Steer Bar Brace

After being secured to the foot, it is brought up parallel to the leg, but if not bent with wrenches so as to fit the angle which the best possible position of the foot forms with the leg, it will not be efficient. The upper end of the bar will either stand away from the leg, as in the illustration, and as would happen at the beginning of treatment, or it will come into contact with the leg before all possible correction is procured, as would happen when over-correction was being attained.

further trimmed and any wrinkles cut out of its inner surface. It may be secured in position by adhesive straps or by a bandage. If straps are riveted to it at its upper end, at the ankle and at the forefoot, it will be somewhat 
easier to remove and to apply. Chloroform may be used as a softening agent and is especially useful if it is desired to re-soften any particular places.

Wood Splint.-A very effective splint, especially for the first few weeks of treatment, is a straight piece of

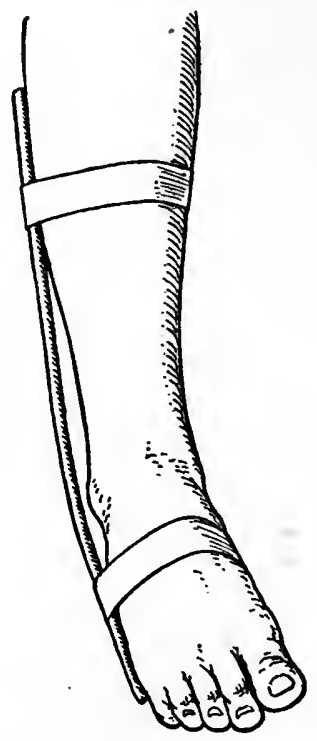

Fig. 55. The Single Steet. Bar Brace

The bar has been curved so as to securely hold all of the correction which it is possible to obtain with its leverage action, and at the same time to lie snugly against the upper part of the leg.

wood, reaching from the knee to three inches below the toes, about an inch wide and a quarter of an inch thick. It is well padded and bound to the foot by a cotton bandage. A small pad is placed opposite the ankle and the upper extremity of the splint drawn over to the leg and 
bound by continuing the bandaging up the leg. The pad at the ankle acts as a fulcrum and will therefore influence the amount of the correction obtained. Too much padding must not be used at first, but it can be gradually increased at successive dressings. If a piece of metal is used instead of the wood, its efficiency can be increased by turning the bar with wrenches as described in the Judson brace.

Plaster-of-Paris. - The plaster-of-Paris dressing is used in the following manner: Strips of cotton wadding one and one-half inches long and the size of a lead pencil are placed between the toes, allowing the ends to extend an equal distance up the dorsum and down the plantar surface of the foot. Two strips of adhesive plaster five inches long and three-eighths of an inch wide are placed along the inner and outer sides of the leg in such a way that the upper half of each adheres to the leg while the lower half extends below the foot and may be turned up and incorporated into the plaster-of-Paris bandage and so prevent its slipping down and off the foot. A flannel or cotton bandage is next applied. This should be one and one-half inches wide. Beginning at the toes it is wound around the foot and leg in such a direction as to tend to unroll the foot. It is put on in circular turns and should lie very smoothly, without wrinkles, and should not be drawn tightly in any turn. The plaster bandage is now applied. It should be of the same width as the cotton bandage and should be applied in the same way as to direction and as to care in not causing con- 
strictions at any turn and in not having any wrinkles. As each turn is applied it is thoroughly rubbed into the one preceding, as in this way the strength of the dressing is greatly increased. After the first two layers are put on, the adhesive plaster strips are turned up and incorporated in the plaster-of-Paris. During the entire procedure it is essential that the foot be retained in the best position; corrected or even over-corrected. The office of the plaster-of-Paris is to retain the foot in the position in which the plaster is applied. That is all. Of itself it does not cause any further correction. After the plaster is applied no further correction should be attempted. There is temptation still further to improve the position while the plaster is hardening, but this must not be done as there are great probabilities that the movement will cause folds and creases and thereby injure the skin. With a little experience a surgeon is able to hold an infant's foot with one hand while with the other hand he rolls the plaster bandage about the leg and foot, his assistant holding the bandage as he passes his hand from one side of the leg to the other.

This dressing should be removed once in two weeks; that on one foot being changed one week and that on the other the next week. In this way the time of each visit is but a few minutes, a valuable point in the treatment of babies, and it also allows for the observation of the plaster applied the week previously.

Points to be observed in the application of plaster-ofParis in these cases are as follows: Do not use too much. 
padding; do not manipulate so severely as to cause any subsequent swelling, which would make a close fit at the time the plaster is applied become a tight one later. The points of the greatest pressure will be on the inner side of the forefoot and heel and on the outer side of the medio-tarsal joint and over the external malleolus. Unroll the plaster bandage smoothly, laying it on rather than binding it on. Hold the foot steadily in the best attainable position, from the beginning until the operation is finished. Three to five layers of the plaster bandage are all that is necessary providing the layers are well incorporated, rubbed. Compress the forefoot between the hands, in a dorsal-plantar direction, so as to assure sufficient room for the toes. Mold the plaster carefully over the instep; this will be of great assistance in preventing the dressing from slipping down and off the foot. One of the objections to a bandage which is thicker than it need be, is that on account of its weight it is more easily kicked off by the baby.

Willard's Shoe.-Willard used a simple arrangement whereby a correcting force can be continuously exerted. A close fitting leather shoe lacing down to the toe has its shank removed or replaced by soft leather, so that free movement may be had between the fore and hind part of the shoe. A stirrup-iron reaching up to the knee is secured to the outer side of the shoe. A stout piece of catgut is attached to the shoe at a spot to the outer side of the little toe and then threaded through an eyelet on a short piece of steel extending forward and 
outward from the bend of the stirrup, and is secured to a piece of elastic which is fastened by its other end to a buckle or a button on the upright at a point opposite the tibial tubercle. The eyelet through which the catgut passes should be so placed that the vertical axis of movement of the medio-tarsal joint shall have the same radius from it as has the point of attachment of the catgut to the shoe. Willard strongly advocated this apparatus and without experience in its use criticism seems ill-advised, but it scarcely appeals to me except for use in very mild cases.

Taylor's Brace.- The Taylor club-foot brace is a very efficient apparatus after the baby is a few months old. It consists of an inside leg-bar with a calf band, jointed below to a foot-piece. The foot-piece has a sole plate with a flange curving up over the first metatarsal and another flange at the inner side of the heel. On the outer side, opposite the heel, is a small extension with a slot through which is passed a strap which goes over the ankle and into another slot on the heel-flange, to hold the heel down against the foot-plate. The joint at the ankle permits one to fit the brace to any degree of equinus. By bending the upright leg-bar any degree of eversion may be obtained. The bending upward and outward of the toe-flange, or the interposition of pieces of felt, will increase the abduction of the forefoot. This form of brace may be used after the child begins to walk, and will be found to meet every indication if it is properly designed and is kept properly fitted. When used as an 
ambulatory apparatus, a piece of wood, made wedgeshaped so that its inner edge will be from one to two inches higher than its outer edge, thus acting as a strong

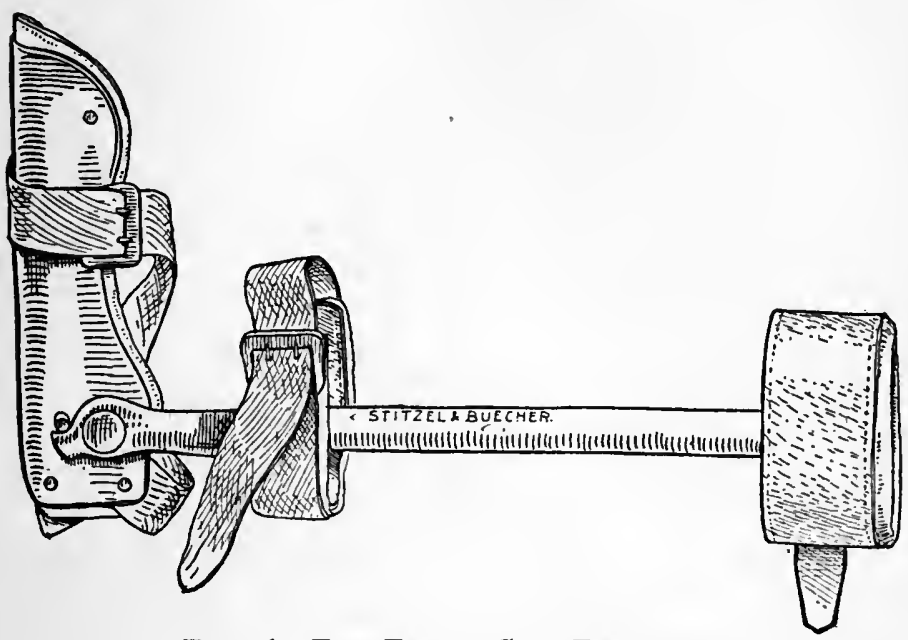

Fig. 56. The Taylor Club-Foot Brace

This illustrates a design somewhat modified from the original, but the principles of mechano-therapeutics upon which it is constructed are essentially the same. The peg-stop at the joint had better be a screw and several holes should be tapped so that by changing the position of the screw the foot-piece can be adjusted to fit the amount of equinus present at the time of application and can readily be altered as dorsal-flexion is increased. With the inside flange extending from the toe to the heel, the flange itself cannot be changed so as to alter the amount of abduction obtainable; abduction must be increased by inserting felt or other material between the flange and the forefoot.

everter of the foot, may be riveted to the under side of the foot-plate.

To design the Taylor brace, a piece of modeling cardboard is desirable, although any other easily cut and easily molded material will answer the purpose. An 
outline of the bottom of the foot is first traced upon the cardboard and then with the pencil the flanges and the extension for the ankle strap are drawn as they would appear if flattened out to the plane of the foot-plate. This tracing is then cut out, the flanges bent up, and

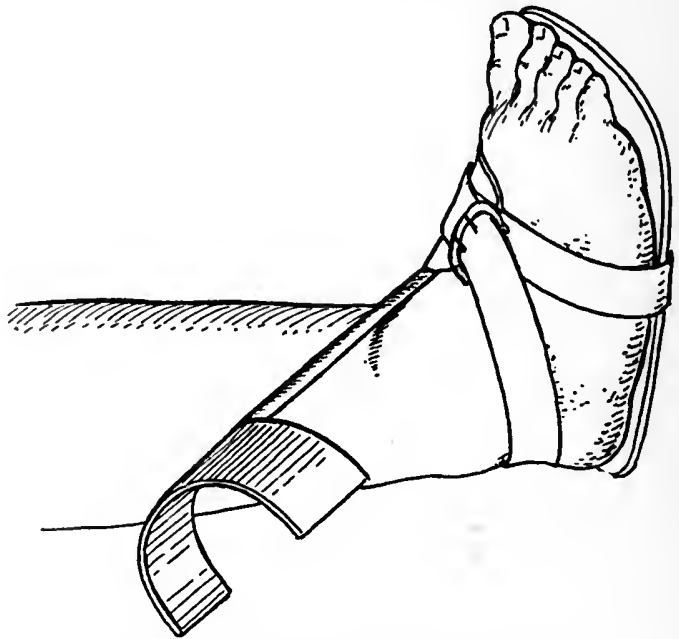

Fig. 57. Taylor Club-Foot Brace

The brace is applied by first securing the foot-piece to the foot in its deformed position. The leg-bar is then brought across the leg, everting the foot, and backward to the line of the leg, and the band slipped about the calf, which corrects the equinus deformity. The flange on the inner side holds the foot in abduction.

the model tried on the foot. It is essential that the foot be held in the best position while the fitting is being done. Alterations are marked and the changes made, and these repeated until the model fits evenly and snugly. Then the leg-bar is cut out, fitted, and marked for the joint with the foot-piece. A model of the calf-band is made 
in the same way. By making deep slots in the forefoot flange, extending from the edge well down into the soleplate, one may more easily alter the completed apparatus as the adduction is overcome. With this model, any worker in metal, such as a tinsmith, can make a perfectly satisfactory brace. A leather worker, such as a shoemaker, can line the brace as desired and make the straps.

Deformity in the Long Bones. - It is seldom necessary to extend above the knee whatever apparatus is used to correct deformity in the long bones. Yet cases will be met with in which the foot seems to be in a good position in its relation to the leg but persists in retaining a position of inward rotation, so that the toes are pointed inward. This rotation may be confined to a rotation at the knee or to an inward twist of the tibia or of the femur or it may be due to a posterior curving of the neck of the femur. To determine this matter, the foot is placed in its normal position with relation to the vertical plane of the body; and the direction of the external face of the great trochanter, to the position of the external epicondyle, and the location of the patella determined; or the foot may be left in its apparently deformed position and the relations of these anatomical points then ascertained. If the vertical plane containing the external border of the patella is outside of the great toe, the foot being dorsal flexed to a right angle and the knee extended, then a twist exists in the shafts of the bones of the leg. According to J. K. Murphy this twist in the tibia and fibula exists in a large proportion of cases. 


\section{I44 DISEASES AND DEFORMITIES OF THE FOOT}

Those due to a twist in the femoral shaft or neck are less frequent. Laxity of the knee ligaments is more likely to prove to be a confusing factor in early childhood than in infancy.

Until the child begins to walk, no other treatment than manipulation is necessary for these causes of inward rotation. The manipulation should consist of untwisting movements performed at the time of the other manipulations. Of course if there is extra lateral movement at the knee joint no exercises are used: usually nothing need be done, but the ligamentous laxity can be improved by immobilizing the knee joint during the day when the child is most active. Whatever apparatus is being used to treat the foot can be carried up so as to include the knee, as is recommended by some orthopedic surgeons in every case of club-foot; but we would remind the reader that the less immobilization required the better is the development, a most important consideration at all times of life, but of especial importance during infancy and youth.

After the child begins to walk, it may be best to correct any inward rotation by continuing the leg-bar of the brace up to a pelvic band. It will be useless to make a brace to include only the thigh. Free joints should be had at the knee and hip.

Osteotomy or osteoclasis should not be performed while the bones are yet soft; after the bones have hardened these operations may be necessary. 
Therapeutics of Early Treatment.- Impatience for results should not lead one to attempt too great a correction within the first few weeks. It is well to remember that no brace or plaster-of-Paris bandage is actively reducing the deformity while it is in place. They are retention apparatuses only. If the foot is not being used for support, there is no influence exerted on any of the structures except in a passive way. By holding the foot in a position which causes a slack in the muscles and ligaments; which are abnormally long in the deformed foot, these apparatuses cause nature to shorten those structures and thus are a powerful means of bringing about a cure; but they are not re-shaping bones nor exerting a corrective force while in situ.

The object to be aimed at in any form of treatment is an exaggerated abduction and eversion. There is no danger of this over-correction becoming itself a deformity. The so-called relapses are much more likely to occur when the correction has been limited to a restoration of the foot to its normal position, because this apparent correction is, in all probability, limited to the soft parts and is hiding deformities of the bones which persist and which, upon the foot's assuming its functions, become aggravated. If this over-correction can be obtained and maintained until the child begins to walk, the best possible result may be expected. The equinus position need not be fully corrected before the end of at least the tenth month (Willard). 
TREATMENT WHEN THE CHILD BEGINS TO STAND

When the child begins to stand, a new element enters into consideration. The weight of the body may be used as a powerful means of aiding correction and, on the other hand, if it is not properly directed, this very force may make the condition much worse. The muscles, ligaments, and bones are all actively used in maintaining the upright position and, if they are employed as in the normal foot, they will tend to become normal in strength, length, and shape. So important is the normal use of the foot in overcoming these deformities that some surgeons reserve their most active measures until the age of walking has been reached. Willard, who believed in beginning treatment at the earliest possible opportunity, said: "Walking in proper position is a mechanical measure superior to any apparatus." Yet Kirmisson believes it to be indispensable that the child should not be allowed to make any attempts to walk until about the age of twenty months. While, therefore, Kirmisson recognizes the potency of walking in altering the feet, he does not think it advisable, for the ordinary surgeon at least, to attempt to direct this force toward a curative measure. We know of no American orthopedic surgeon who agrees with him. No amount of massage or manipulations can be the equal of even a slight functional activity.

Although a plaster-of-Paris bandage can be applied so as to hold the foot in the corrected or over-corrected po- 
sition and the child in standing and walking will exert the force of the weight of the body especially toward reshaping the bones; yet so much more can be obtained by every normal activity which is encouraged, be it of muscle, bone, or ligament, that it is advisable to use a brace which allows of more normal use of the parts of the foot than can any solid casing. The Taylor brace is a most suitable one for this stage of treatment.

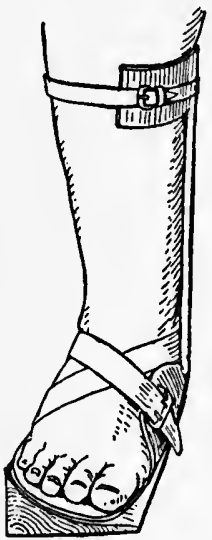

Fic. 58. Taylor Club-Foot Brace Applied

This holds the foot everted and abducted and at a right angle to the leg. A wedge piece of wood, extending the length of the footpiece, holds the over-correction when used as an ambulatory brace.

As soon as the foot begins to assume its functions, the lime salts are deposited much more rapidly in the bones and they become ossified in whatever shape they are allowed to maintain. Whereas up to this time the deformity was due to the soft structures more particularly, the bones now assume a continuously increasing impor- 
tance as a factor in obstructing correction or, under proper treatment, in rendering a cure permanent.

It may be well to recapitulate the most marked pathological changes in a congenital equino-varus, untreated and walked upon. The astragalus, rotated far forward, in an exaggerated normal position as it is during infancy in this condition, will have an increased growth of bone on that part of the trochlear surface which protrudes from the mortise, and also an increased development of the peroneal tubercle. The posterior part of the head and the adjoining part of the neck will form the articular surface for the scaphoid. The anterior part of the head, left uncovered by the new position, more posterior and inferior, assumed by the scaphoid, may present a ridge of bone marking the anterior limits of the new articulation. As the narrowest part of the trochlear surface is, in this position of hyper-extension, presented in the tibio-fibular articulation, there may be some slight rotation inward of the astragalus, thus increasing the position of adduction. Various changes in the neck have been described by different observers. The two changes which are of paramount importance to the surgeon are the alteration of the head, which may prevent reposition of the scaphoid to its normal relation with this bone, and the changes which may positively interfere with the rolling back of the trochlear surface into its mortise.

Tubby describes a new non-articular surface on the astragalus. It is nearly quadrilateral; its upper and inner border is coterminous with the outer part of the 
anterior and lower border of the tibia. The upper and outer border correspond with the anterior edge of the external malleolus. The outer border blends with the prefibular tubercle and the inner part passes gradually into the neck and head.

The os calcis, following the position of the astragalus, has its antero-posterior axis directed forward, downward and inward. It may be so far twisted inward that its tuberosity is in contact with the fibula and may form a joint with the external malleolus. The articular surface for the cuboid may have an excrescence of bone on its outer part, developed in consequence of the cuboid's being partly dislocated inward by the adduction of the forefoot; and the weight of the body resting on this surface and this new bony growth may offer an obstruction to the perfect replacement of the cuboid. In very severe cases there may be a twist of the bone itself on its long axis. The most serious obstacle offered by the os calcis to reduction of deformity is the bony excrescence on the articular surface for the cuboid.

The contractures of the soft tissues become more firmly established the longer treatment is postponed after birth. Those which may have to be divided, are: the tendo Achillis, most frequently; the two tibials, next so; and in untreated cases of two years or more, less frequently in younger cases, the ligaments comprising the capsule of the astragalo-scaphoid joint and the plantar fascia. In markedly severe cases all the soft parts on the inner and plantar surfaces may need division. The posterior 
ligament of the ankle joint, if it needs division, is divided at the time of tenotomy of the tendo Achillis, as explained under that operation.

\section{CONGENITAL TALIPES EQUINO-VALGUS}

This deformity occurs in Whitman's table, in 1.7 per cent. of all cases of congenital talipes. The heel is raised and the foot can not be brought to a rightangle with the leg. The movements through the other two axes, the longitudinal, sub-astragaloid, and the vertical, mediotarsal, are the reverse of those found in equino-varus. The plantar surface turns outward, the outer border of the foot being raised, and the forefoot is abducted. Treatment is correction by manipulation and by apparatus or plaster-of-Paris. The difficulties met are seldom difficult to overcome.

\section{CONGENITAL TALIPES EQUINUS}

This is a rare deformity. Treatment is by manipulation. Seldom is any brace or operation demanded. A simple ankle brace with a right-angle stop may be applied. The only operation that could under any circumstances be necessary would be division of the tendo Achillis. No writer records a case where it was necessary to divide the posterior ligament.

\section{CONGENITAL TALIPES VALGUS}

In frequency of occurrence, this deformity stands next to talipes equino-varus. In all probability there is some 
elevation of the tuberosity of the os calcis. Adams considered this to be the case. In infants it is by no means easy to determine the exact degree of dorsal flexion, and therefore, what one might term pure valgus another might classify as equino-valgus. However that may be, it is of little importance as the equinus must, in any case, be slight and the valgus is the pronounced deformity. In examining for this condition, it should be borne in mind that the arch of the infant's foot is not developed and the normal flat-foot must not be allowed to lead one astray. On the other hand, as the normal foot at this age is held in a position of slight inversion and adduction, the eversion and abduction are the more noticeable.

Treatment: If unaccompanied by other abnormalities, the treatment should be along the lines already laid down. Frequently, however, it is associated with absence of the fibula. In such cases, Tubby found arthrodesis of the ankle joint necessary and not always successful.

\section{CONGENITAL TALIPES VARUS}

In frequency this stands next to pure valgus. Tubby found 44 cases in 3 I I of congenital talipes. Treatment is the same as that described for the varus under equinovarus.

\section{CONGENITAL TALIPES CALCANEUS}

This deformity in a severe degree, is rare. Upon an attempt to plantar flex the foot the tendons on the dor- 
sum of the foot may be felt to tighten. Three degrees are described: that in which the foot can be plantar flexed to a rightangle; that in which it can be plantar flexed to an acute angle with the leg; and that in which it lies almost parallel with the leg. There is usually some rotation on an antero-posterior axis so that it may be called calcaneo-valgus or calcaneo-varus. Walsham states that most of his cases had the sole looking inward. Plantar flexion of the metatarsal-phalangeal joints is usually present. More rarely they are fully extended. According to Adams, rigidity of the knee joint is more frequently associated with this deformity than with any other form of congenital talipes.

The pathology, in slight degrees, is limited to a shortening of the anterior ligaments and muscles. In more severe cases there may be elongation of the posterior ligaments and muscles, and there may be some bony changes, confined mostly to a prolongation of the articular surface of the astragalus forward. The os calcis may be almost vertical. Hoffa described the cuboid as being on almost the same level as the head of the astragalus.

Treatment is by manipulation and braces. Division of the shortened structures is necessary in the severest cases.

CONGENITAL TALIPES VALGUS AND VARUS

In fourteen cases, thirteen were valgoid and one was varoid (Walsham and Hughes). In thirty-four 
cases, twenty-one were valgoid and two were varoid (Tubby).

\section{CONGENITAL TALIPES PLANUS}

The dome is developed only with the development of the muscles at the time the child begins to stand and walk. If the bones retain their infantile relations, no arch is formed and a flat-foot exists. A better term for this condition is infantile flat-foot.

\section{CONGENITAL TALIPES CAVUS}

This is sometimes met with but is very rare.

\section{CONGENITAL TALIPES VALGO-CAVUS}

CONGENITAL TALIPES EQUINO-CAVUS

Both of these conditions are described but are extremely rare.

OPERATIVE PROCEDURES IN THE TREATMENT OF CONGENITAL CLUB-FOOT

Wrenching.-This consists of forcible manipulations with the patient under an anesthetic. Usually the hands are the only instruments needed, but a wedgeshaped fulcrum may oftentimes be used to advantage, and more rarely, a wrench. Although a break in the skin will occur only through an accident, the preparation of the feet as for an open operation is advisable. When the patient is thoroughly anesthetized, the foot is grasped 


\section{54 DISEASES AND DEFORMITIES OF THE FOOT}

in the same way as it is for ordinary manipulations. Whatever the deformity may be, the equinus, if such exists, is to be left to the last attack. Steady, strong pressure is not the aim. Much more will be gained, and that with less danger and in less time, if the force is applied momentarily. With the aid of assistants the power can be confined to the desired joint and there will be no danger of fracturing bones. A foot which is perfectly rigid, even with the patient fully under the anesthetic, can usually be rendered more or less mobile; but perseverance and considerable strength are necessary. It may take ten minutes, or thirty, or forty, or even an hour.

The object of using an instrument, such as a wrench, is to obtain more leverage. If the surgeon and his assistants have not strong arms and hands, a wrench will be of great help. It should never be employed simply to gain time. It is not free from danger, such as severe bruising of the soft parts, which may interfere with the ultimate result, and the breaking of bones. Several patterns of wrenches have been made; such as the Thomas wrench, which is the best known, and the Myers wrench. Before application, the foot is wrapped in a towel so as to lessen the friction between the wrench's jaws and the skin, should there be any slipping; and it is impossible to avoid some slipping. Rubber tubing slipped over the jaws is also useful for this purpose. Both the tubing and the towel or any other sort of padding also serve to equalize the pressure: as they will spread out on all 
sides from the prominent points of contact between the jaws and the foot, they will fill up the depressions between these points and thereby extend the surface receiving the force. As an illustration of the daring, or

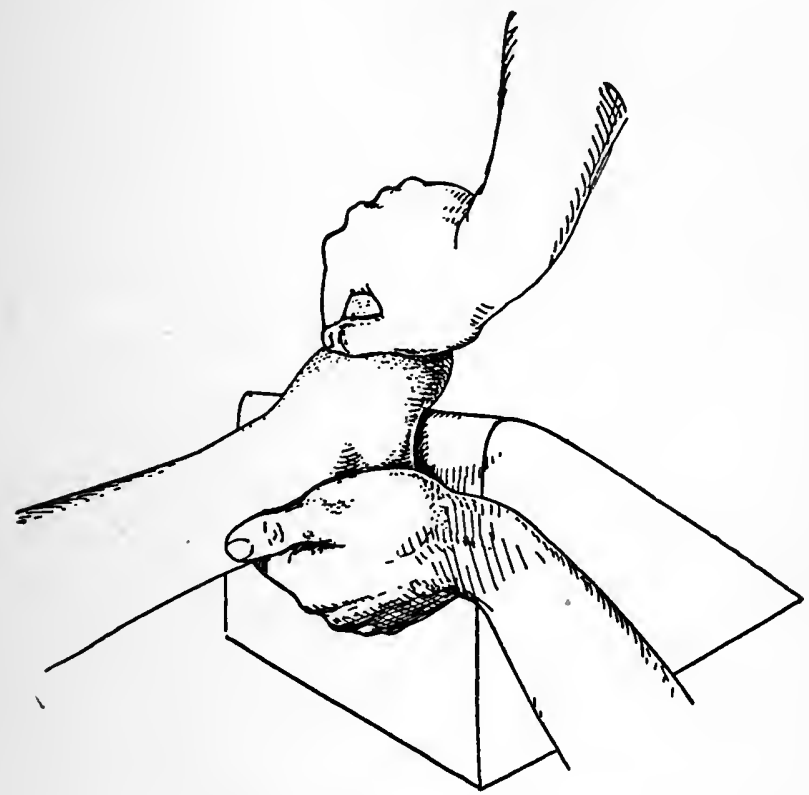

Fig. 59. The Use of a Wedge

Overcoming adduction by the use of a wedge. There should be some padding between the foot and the wedge.

callousness, of some surgeons in the past, some of the devices for wrenching the foot are most interesting.

A wedge-shaped piece of wood, padded along its apex, is often used to supply a fulcrum in manual manipulation. It is especially of service in correcting abduction 
or adduction, as it can be placed in line with the vertical axis at the medio-tarsal joint.

After all the correction possible has been obtained, the question may arise as to the advisability of dividing some of the contracted tissues. It is usually better to postpone the use of the knife until several manipulative operations have been performed at intervals of about six weeks; for the improvement directly resulting from manipulation is increased by use, weight-bearing, in the improved position. The peculiarities of each particular case must, however, determine this question. It should be remembered that if alterations in the shape of the bones is necessary for a cure, such alterations can not be expected to result immediately from any re-position of the parts. Until about the eighth year there is no doubt that the bones can be influenced to change their conformations: after that age it is much more difficult, but, I believe, quite possible. During manipulation the shape of the bone is altered not at all, unless, possibly, it might be in infancy. Manipulation stretches the soft structures so as to permit the placing of the bones, if not too greatly altered in shape, in positions, in relation to each other, which are normal or more nearly normal. If the value of manipulation ended with the operation, it would often be worse than useless. The manipulated foot in most cases of confirmed deformity would be weaker than it was before operation and would probably be painful.

The object of manipulation is to place the structures, 
as nearly as possible, in their normal positions and then to take advantage of those physiological laws which have been described by Wolff and by Davis.

Wolff's Law._- Every change in the form and position of the bones, or of their function, is followed by certain definite changes in their internal architecture, and equally definite secondary alterations of their external conformation in accordance with mathematical laws."

If weight is borne on the outer side of a bone, for instance, the laminæ laid down in that part will be such as to receive the pressure and will differ from the laminæ laid down in the other parts which do not bear weight. Now were the weight shifted so that the function formerly assumed by the outer part of the bone was borne by the inner part, then the style of the internal architecture would become exactly reversed; the weight-bearing part would be altered to meet the new function. Therefore it will be understood that manipulation must be followed by use in the new position. If the bones in their new relations to each other are not used, neither their internal architecture nor their external configuration will be changed.

Davis's Law.-_ Ligaments, or any soft tissue, when put under even a moderate degree of tension, if that tension is unremitting, will elongate by the addition of new material; on the contrary, when ligaments, or other soft tissues, remain uninterruptedly in a loose or lax state, they will gradually shorten, as the effete ma- 
terial is removed, until they come to maintain the same relation to the bony structures with which they are united that they did before their shortening. Nature never wastes her time and material in maintaining a muscle or ligament at its original length when the distance between their points of origin and insertion is for any considerable time, without interruption, shortened."

These physiological laws are simply expressions of the influence of work, rest, and fatigue on metabolism and the resultant hypertrophy and atrophy.

With the bones of the foot in an altered position as the result of manipulation, and the foot used in weightbearing, changes will occur in the bones, muscles, and ligaments. Perfect functionating, freedom of movements, can not be allowed immediately, as even if the contused condition of the foot, as a result of the manipulations, did not demand rest, the inuscles and ligaments which have been relaxed and made slack must be allowed to shorten, or more strictly speaking, be allowed to rebuild themselves as shorter structures; else the bones would soon assume their former relations.

If the case is one of long standing and much change must take place in the bones; absorption here, building up there, and the formation of new facets, then a much longer time will be necessary to give stability to the new position than would be demanded by a case where the anatomical changes were slight or were limited to the soft tissues. It is probable that an imperfect appreciation of the importance of this element of time has been 
the cause of deformed feet, apparently cured, suffering a relapse, in many such unfortunate instances. The exact

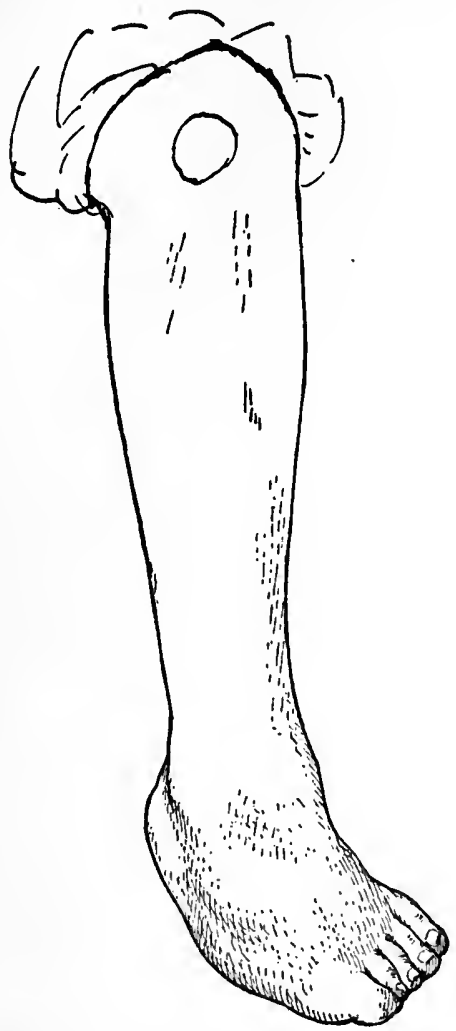

Fig. 60. Relapsed Congenital Talipes Equino Varus If an over-corrected position is not maintained until, with growth, all tendency to resumption of the deformity is gone, a relapse is certain to occur.

time necessary for these structural changes to take place is hard to determine. It doubtless varies with the age 
of the patient and with the physiological activity, depending on constitutional conditions and functional use, as well as with the amount of structural changes necessary to effect a cure.

Let us return to the operation. Before the patient comes out of the anesthetic, the foot is placed in plasterof-Paris. Strips of cotton batting are placed between the toes to prevent their being crumpled up and to keep them flat, then batting is wrapped around the foot and leg as high as the tibial tubercle. This batting must be thick enough to allow for the swelling which will result from the manipulation. Usually it should be threefourths of an inch or more thick. As the foot is to be stood upon and walked on, the bottom of the plaster must be made extra strong. A good way to reënforce it is to make a sole, by doubling back and forth upon itself a three-inch plaster bandage, making a pad the length of the foot, and then binding it on with another plaster bandage and rubbing it well until thoroughly set. By making this sole thicker in one part than another, under the inner side if a valgus has been corrected or under the outer side if a varus has been corrected, or under the forefoot if the deformed condition was one of equinus and under the heel if there was a calcaneus, a further corrective force is obtained. When stepped upon, the thicker part of the sole will push up on the part directly above with more force than will be felt by the other parts of the foot.

Over-correction should be the aim of the operator and 
the plaster bandage should be applied with the foot in the over-corrected position. A case of equino-varus should be put up in a position of calcaneo-valgus, or a position approaching it as nearly as possible.

\section{TENOTOMY}

History.- The history of subcutaneous tenotomy is an interesting one. Before the day of antisepsis, this operation assumed an importance which has been equaled in few instances, I venture to state, in the discovery of any new surgical procedure. To Stromeyer is due all honor for conceiving it and putting it into practice, and to Little is due all honor for giving it a wide introduction, and to Detmould for bringing a full knowledge of it to this country.

Tenotomies, with a more or less open wound, had been done long before Stromeyer's day. Many knew of Stromeyer's work before Little visited him as a patient and a student. Subcutaneous tenotomies had been done in America before Detmould returned from his studies abroad. The question of priority is raised with every invention and discovery, but subcutaneous tenotomy will always be associated with Stromeyer and Little in the annals of surgery; and the profession in this country should remember the name of Detmould in this connection. Dr. Dickson of North Carolina performed the operation in 1835 and Dr. Smith of Baltimore in 1836 , while Detmould did not perform the operation in New York until 1837 ; but his is the credit for advocating and 
teaching the operation so that its wonderful possibilities became widely known and countless thousands of cripples have been greatly benefited.

Value.- In this day of aseptic surgery, some think that subcutaneous tenotomy is evidence of poor surgical skill, that an open operation is better art. On the contrary, it requires greater skill cleverly to divide tendons subcutaneously than through an open wound. Not only must the operator's knowledge of the anatomy of the part be perfect but the fingers must be very deft and sensitive. The open method, in spite of the advancement of aseptic surgery, is, in many instances, disadvantageous to the best results. Cicatricial tissue is to be avoided as much as possible in the correction of any deformity: not only can it, of itself, cause a deformity by its inevitable contraction, but it is a most difficult tissue to stretch. Subcutaneous tenotomies are much quicker in the doing, there are practically no wounds to be dressed and there is no interruption in the treatment by apparatus. Furthermore, laboratory experiments have demonstrated that new tendon is produced only by the tendon sheath and the peritendoneum, the severed ends not assisting. If the sheath is left intact, the width of the gap between the severed ends can not be of grave importance, providing the gap is not so great as to allow of the sheath being entirely collapsed and drawn tight like a whipcord. So, even if an open wound is made to reach the tendon, it is better surgery not to open up the sheath, if simple tenotomy is to be performed, and thus 
no gain is made over the subcutaneous method except to allow of seeing that none of the neighboring structures is injured, an unnecessary precaution for the skillful surgeon.

Tendo Achillis. - This was the first tendon to be divided by the subcutaneous method. After preparation of the site, a narrow-bladed tenotome is inserted close to the inner border of the tendon, opposite the thickest part of the internal malleolus, where the tendon is the narrowest. The foot is held in slight dorsal flexion so as to bring the tendon into relief; and the blade, held flat to the tendon, is passed into the posterior part of the sheath and through to the outer border of the tendon, where it may be felt by the finger. The blade is now turned with its cutting edge toward the tendon and the foot dorsal-flexed to its limit. If the knife has been held firmly this tightening of the tendon will sometimes be sufficient to divide it; but if not, then a few sawing-like movements will be all that is necessary. A finger held over the tendon and pressing down upon it will inform the surgeon of the progress the blade is making. Some surgeons have advised that the last few fibers be torn rather than cut, so as to leave something of a bridge for the better growth of the new tendon; but this (by our fuller knowledge of tendon regeneration), is now known to be unnecessary. The skin puncture can be made with a sharp-pointed bistoury if desired and then the bluntpointed one inserted; but it is almost impossible to avoid cutting the entire breadth of the posterior part of the 
sheath by such a procedure and if the sharp-pointed knife is inserted through the skin on into the sheath and then withdrawn and the blunt-pointed one then used, it is not always an easy matter to find the opening in the sheath. It is much the better plan to use the sharp-pointed tenotome from start to finish. It should not be necessary to withdraw the blade until the operation is completed.

The object of subcutaneous tenotomy is lost if there is more than one small puncture in the skin and in the tendon-sheath. The tenotome should have a short, narrow blade with a long shank. There are many shapes and sizes on the market, but there is none so good as one made by grinding down the blade of an ordinary scalpel. The blade should be short so that its cutting edge will not overlap the tendon and cut the sheath. It should be narrow so that it will not make a long wound in piercing the skin and sheath. It should have a narrow shank and a long one, so that the skin puncture will not be needlessly enlarged during the operation.

The wound is dressed with a piece of sterile cotton dipped in a fifty per cent. solution of alcohol. The correction permitted by the operation is immediately accomplished and if nothing more is to be done, a plaster-of-Paris dressing is applied. Care should be taken that the dressing over the gap in the tendon does not press into it and thereby interfere with the generation of the new tendon.

Indications. - Tenotomy of the tendo Achillis is indi- 
cated in a marked equinus where the tautness of the tendon on any effort at dorsal flexion proves it to be one, if not the only one, of the obstructions to normal movement. An open operation is the method of choice whenever the lengthening of the heel-cord must be two inches, as the sheath will be badly lacerated if subjected to greater extension, and also in those cases where previous operation has resulted in much scar tissue. Scar tissue will not lend itself to extension but will tear when much force is applied; and if the scar tissue includes the skin a large gap may result which will have to heal by granulating from the bottom, thus almost certainly precluding any resulting function on the part of the gastrocnemius. In these cases and also when an exact amount of lengthening is desirable, a splicing of the tendon is the procedure of choice, with a plastic operation for the removal of scar tissue. The tendo Achillis should never be operated upon until all other deformities have been corrected in so far as is possible.

Posterior Ligament.- Lying between the tibia and the astragalus this ligament may prevent dorsal flexion after division of the tendo Achillis or it may have been the only obstruction to correction of the equinus. Its division is usually effected after tenotomy of the heel-cord by turning the cutting edge of the tenotome directly forward toward the joint, when it may be divided without danger to other structures. Broca, however, preferred to tenotomize the tendo Achillis by the open method, not on account of any danger in that operation by the sub- 
cutaneous method, but to be able to cut this ligament more easily.

To determine what structure, whether muscle, ligament, or bone, is preventing dorsal flexion, a most careful analytical examination is necessary. It is a cruel disappointment to expect a simple tenotomy to cure an equinus and to find that the deformity persists after the operation. That the posterior ligament is the chief factor may sometimes be ascertained by noting that the tension of the tendo Achillis is not complete in forced dorsal flexion, and that bony obstruction alone has a different "feel" as the limitation of movement is reached. It is well to give a conservative prognosis in all doubtful cases.

Tibialis Posticus.- Tenotomy of this tendon was first done by Stromeyer in 1834 and by Little in 1842 . It takes more skill than does division of the tendo Achillis but, when indicated, no hesitancy should be felt by the surgeon in undertaking it. By some surgeons, notably Broadhurst, it is considered as demanding division in nearly every case.

This operation can be done in either one of two locations before it reaches the end of the tibia and while it is still in the groove of that bone, or just before its insertion into the internal cuneiform. If it is to be divided alone, or with the flexor longus digitorum, the first position is the one of choice. If the tibialis anticus and the astragalo-scaphoid capsule are to be divided at the same operation, then the latter situation is preferable. In in- 
fants it is well to follow Little's instructions; the tenotome is entered one and one-half inches above the tip of the internal malleolus, at a point midway between the front and back of the leg. It is pressed down until the posterior edge of the bone is felt. The tendon is relaxed by exaggerating the deformity and the blade is slipped between the tendon and the bone. Its cutting edge is then turned toward the tendon and division usually effected simply by rendering it taut. The assistant holding the foot may feel the snap more plainly than the operator and will notice the advancement toward correction.

The greatest danger in performing this operation is that of wounding the posterior tibial artery. Should this happen it is not necessary to cut down on the artery and tie it off; dressing with a pad will stop the bleeding. The presence of an abundance of bright red blood on the withdrawal of the knife and the sudden blanching of the foot, are the indications of this accident. Should this happen the foot should be put up in the deformed position for five days and correction made after that time.

The tibialis posticus does not, in equino-varus, pursue its normal course. Its attachment to the scaphoid and to the internal cuneiform will cause its direction to be determined by the altered position of these bones. It may take a directly downward path, beneath the malleolus; or it may go slightly forward; or, according to one writer, it may take a course slightly backward. How- 
ever, it must, in any case, cross the astragalo-scaphoid joint and will therefore be divided at the same operation for the divisions of the ligaments of this joint.

Astragalo-Scaphoid Capsule.-As described by Parker, this is "made up, above and internally by a blending together of the inferior astragalo-scaphoid ligament with fibers from the anterior ligament and the anterior portion of the deltoid ligament of the ankle joint, below the fibers from the calcaneo-scaphoid ligament. To these are united fibrous expansions of the tendons of the anterior and posterior tibial muscles. This dense structure is fortunately quite subcutaneous and so placed that it can be divided without risk to other structures." Its division is effected by inserting the tenotome close to the anterior border of the internal malleolus, passing the blade on the flat, downward, just beneath the skin, for about one-half an inch or until the tip reaches the lowest plane of the scaphoid, and then turning the edge inward and cutting directly into the astragalo-scaphoid joint.

Flexor Longus Digitorum.- This tendon may be divided at the same time as the tibialis posticus by pushing the tenotome a little deeper and including both tendons.

Tibialis Anticus.- This may be divided alone as it lies in front of the ankle joint. It will be found displaced inward.

Peroneus Longus and Brevis.-Both may be divided as they lie behind the fibula, about an inch and a 
half above the tip of the external malleolus. The tenotome is passed on the flat between the tendons and the bone, and then the edge turned outward and the tendons divided.

The tendons of the extensor longus pollicis and the extensor longus digitorum may be divided anywhere between the annular ligament and their points of insertion, due care being taken not to wound the anterior tibial artery which lies between the tendon of the extensor longus pollicis and the tendon of the extensor longus digitorum. If all the tendons of the latter muscle are to be divided it is better to cut the tendon before it separates into its divisions.

Plantar Fascia.- The plantar fascia and the muscles in the sole of the foot may be reached through a puncture at about the middle of the length of the internal border or at a point posterior to this; about at the juncture of the middle and the posterior thirds. In the latter situation the scar tissue is less likely to be troublesome and the fascia and the muscles are in a more compact bundle and therefore more capable of thorough division. After the skin puncture is made the tenotome may be passed between the skin and the fascia and the incision made toward the bone; or it may be passed deeply, under the constricting bands, and the cutting done toward the skin. Whichever procedure is followed, the operation must be thorough, no band of fascia or contracted muscle must be left. After the most prominent ones have been divided, abduction and dorsal flexion of the forefoot by 
the assistant will usually bring the deeper ones into relief.

Danger of Aneurism.-In performing the operation of tenotomy, some surgeons advise the use of a bluntpointed bistoury after the skin puncture is made, so as to avoid the pricking of an artery; as a slight prick may result in the formation of an aneurism while a free division of an artery will cause little or no trouble.

Indications for Tenotomy.-In determining whether or not tenotomies are indicated in any case of deformities of the foot, the following points may prove useful:

In infants all the tissues will yield to manipulation. Through faulty manipulation, from whatever cause, one or more tendons may subsequently need to be divided.

Improvement in congenital cases should be steadily progressive. When improvement is arrested, there should be no hesitancy in operating upon the offending structures.

When the child begins to stand, the foot must be in a normal, or better, in an over-corrected position.

Tenotomy of the tendo Achillis should not be performed until all deformities except the equinus are corrected.

In congenital cases of club-foot the tendons are the chief cause of the deformities at birth; the ligaments become factors after a few months, the bones assuming a constantly increasing importance after the first year. Little advised tenotomies at about the eighth or ninth 
month, Adams at about the second month, and Broadhurst at an early period even in cases of slight degree.

In infants the plantar fascia is seldom a serious obstacle to correction.

The tendon of the tibialis posticus is of more importance in preventing correction of the varus than any other one tendon, the tibialis anticus being the next in importance.

The astragalo-scaphoid ligament or rather the capsule which includes this ligament and part of the deltoid as well, needs division much more frequently than does any other ligament.

In adults there are certain cases which yield to tenotomy alone. Broadhurst relates a case, fifty-two years old, "having one of the most severe forms of congenital equino-varus that I ever saw," so far relieved by tenotomies that he could walk with the sole flat on the ground and free ankle movement. Also a case seventy-three years old, cured by tenotomies only, so that " no vestige of deformity remained." However, the more a deformity is used the greater will be the changes which confirm it and we should therefore always expect to find more or less marked changes in the bones in these old cases.

In adults, the mere presence of deformity in the foot is not always sufficient reason for attempting to correct it. E. Duval relates a case, quoting Malgaigne, of an adult with congenital club-foot who could walk, run, and ride. He was operated upon and afterward it took him six hours to walk a distance which he could have covered 
in one hour before the operation. A Dr. Mazet himself suffered from congenital club-foot, but attended to a large practice. He was operated upon and found himself worse off than before. After a second operation he was forced to give up walking altogether and to depend entirely on his carriage. These unfortunate results are instructive as a warning to be chary of a too optimistic prognosis in confirmed cases of long standing. However, tenotomies are seldom followed by a worse condition than that for which they were done, and usually some improvement, if not a perfect cure, results.

Phelps' Operation.-This is essentially a division by open operation of all the resistant tissues on the inner side of the foot. At first it was limited to the soft parts, but later, osteotomy was done if necessary. For some years the operation was performed quite generally, here and abroad, but it has now fallen into disuse except in some specially indicated and unusual cases. The soft parts can usually be divided quite as well by the subcutaneous method and if bone operation is necessary, it had better be done through a flap incision.

\section{BONE OPERATIONS.}

Indications. - While the bones of the foot are plastic they readily yield to any force. This plasticity lessens with age, until about the eighth year, when their ossification is completed. Pressure and movements, before this time is reached, determine their conformation and the extent of their articular surfaces; while afterwards these 
forces act very slowly indeed in their influence on boneformation. This statement applies with equal force to the formation of deformities as well as to their correction.

Until the age of weight-bearing arrives, pressure and movements are determined solely by the muscles and ligaments. After weight-bearing begins, the influence of the muscles and ligaments in directing pressure and limiting movements continues, unless they are put at rest by an immobilizing splint such as a plaster-of-Paris dressing.

If operation on the soft parts permits of correction of deformity, the placing of the bones in their normal relation to each other, whether or not inter-articular cartilage perfectly intervenes; and if functionating takes place, then a cure may be expected. On the other hand, if correction of deformity follows operation on the soft parts but with much separation of bony surfaces, a cure is not likely to result. New articular surfaces will not form if the surfaces are not in contact and moving upon each other; nor will bony growth take place if the stimulation of weight-bearing, or some force transmission, is not present; nor will exuberant bone be absorbed by friction and pressure if friction and pressure do not exist. If the separation of bony surfaces after operation and correction of deformity is slight, then absorption of the fulcrum which is holding the surfaces apart may result in the closing of the gap and the establishment of a permanent cure. 


\section{I74 DISEASES AND DEFORMITIES OF THE FOOT}

Bones to be Attacked.- The bony obstructions to the correction of congenital equino-varus, the most common of the congenital varieties requiring operative interference, are confined practically to the astragalus and the os calcis. The astragalus presents a deformed head and an exuberance of bony growth in front of the articular facet for the fibula. There may also be a ridge of bone across the trochlear surface effectually preventing dorsal flexion. The os calcis presents on the external part of its anterior surface, which is normally occupied by part of the articular surface for the cuboid, an exostosis which mechanically interferes with the reposition of the cuboid to its normal location.

Astragalectomy.- By total removal of the astragalus, all bony obstruction to correcting inversion and equinus are abolished, but the anterior part of the os calcis may still prevent abduction. If the equinus is the chief deformity, the partial removal of the astragalus may be performed, as recommended by Tubby: "If the foot itself is entirely straight with the leg, and the deformity is only at the ankle joint, partial removal suffices. But if inversion is combined with absence of dorsal flexion, the whole bone must be removed." In partial removal he takes away only the upper half of the bone.

Astragalectomy was first performed by Lund in 1872 . The incision is made on the outer side of the foot, extending from behind the external malleolus, about an inch above its tip, downward, and forward to the fifth metatarsal. After elevation of the soft parts, the exact 
positions of the tibio-tarsal and of the astragalo-scaphoid joints are determined and the astraglo-scaphoid ligament is divided. The detachment of the external lateral ligaments will permit sufficient loosening of the bone to allow of its being seized with bone-forceps and so twisted that the internal lateral ligament may be reached with knife or scissors. After that the interosseous ligament is divided and the bone removed. Sometimes it may be easier to remove the bone in two sections, the head and neck being chiseled off before the body is attacked. If after excision of the astragalus, it is found that abduction is impossible, the exuberant bone on the os calcis which is blocking reposition of the cuboid, must be chiseled away. This can be done through the incision already made. It is seldom necessary to disturb the integrity of the cuboid, and if this is not done, a serviceable joint will result.

Partial Excision. - It would seem that instead of removing the entire bone, it should be possible to clear away the obstructions to reposition and leave the body of the astragalus undisturbed. If one can satisfy himself, by his examinations and radiographs, that the obstacle to correction is confined to the head and neck, the excision of this part may be sufficient or this operation combined with resection of the head of the os calcis. This was the procedure advised by Broca in both the child and the adolescent. After ten years of such practice, he said that the facts disprove the theory of its interference with osteogenesis. 
Ogston's Operation.-This operation is devised for the correction of congenital deformities of the foot. It consists of the removal of the ossified center or kernel of the deformed bone and the consequent reduction of the bone to an entirely cartilaginous state permitting of immediate re-shaping of its external configuration. Ligaments, muscles, and joints are left undisturbed. It is applicable until after the sixth year, or until the ossification has not advanced too far when, of course, the operation would be tantamount to excision.

For congenital equino-varus the incision is curved from just in front of the external malleolus, forward to the dorsal aspect of the calcaneo-cuboid joint, with the convexity toward the sole. An incision is made into the head of the astragalus, and with a Volkmann spoon the ossified center is scooped out. The body of the astragalus is treated in the same way, and if necessary, the same treatment is given to the os calcis and to the cuboid. A radiograph will aid in determining whether the growth of the bones has passed beyond the stage when this operation is feasible.

Cuneiform Tarsectomy.-This is the removal of a wedge-shaped piece from the tarsus, irrespective of the exact bones operated upon. The foot being considered as a solid mass, the wedge is removed to permit the restoration of the contour regardless of the exact bones and joints involved in the operation.

Multiple Cuneiform Osteotomies.-Hoke has very successfully treated a number of relapsed and untreated 
cases of congenital equino-varus by carefully planned cuneiform osteotomies of several of the tarsal bones, including the internal cuneiform, cuboid, os calcis, and the astragalus. He also shifted the external malleolus forward by fracturing the fibula one and one-half inches above the tip of the malleolus. 


\section{CHAPTER VII}

\section{POTT'S PARAPLEGIA. CEREBRAL PARALYSIS}

PARALYSIS COMPLICATING POTT'S DISEASE

As the majority of these cases recover and as during its presence no one would think of allowing any attempt at using the feet, it is necessary only to prevent passive stretching of the muscles and ligaments of the foot until their normal strength has returned. The foot should be held by some simple retentive apparatus at a right angle to the leg. A padded tin, gutter splint is easy to construct and meets all the indications. No passive exercises or massage should be prescribed during the period in which the reflexes are markedly active.

\section{SPASTIC PARALYSIS}

As this condition is usually such as to demand as much or more attention to other parts of the body than to the feet, a general discussion will not be attempted, the reader being referred to works on general orthopedic surgery. Sometimes, however, the foot in hemiplegia, or both feet in diplegia, will present the most noticeable abnormality and therefore, treatment as directed toward the feet will be outlined. 
Diagnosis. - The most marked spasm is usually in the gastrocnemius and the soleus, producing equinus, but the inverters of the sole and the adductors of the forefoot are also involved and a talipes equino-varus may exist, with the patient, if able to walk at all, walking on the outer border of the forefoot or even on the dorsum. Although the non-spastic muscles are more or less paralyzed from the constant stretching to which they are subjected, atrophy of the leg is not very noticeable; doubtless hypertrophy of the over-acting muscles hides much of the atrophy.

Examination.-If one grasps the foot firmly and attempts to restore it to its normal position, resistance will be met, which will yield, much as would a stout rubber band, to a steady pressure. This is called "lead pipe contracture" by Weir Mitchell. The deformity is at once resumed upon removal of the pressure.

Treatment.-In an untreated case it is impossible to determine in advance how much may be expected from such measures as look to the relief of the stretching of the non-spastic muscles and the restoration of their power. A brace should be designed to hold the foot in the best possible position without pain. Such a brace will have to be worn inside the shoe - as the strength of the spastic muscles would distort the shoe in an outside ankle brace. Massage and unassisted active movements of the muscles which have been the subject of the stretching, may be practiced three times a day, but such treatment must absolutely be limited to these muscles. 
Sometimes the spasms are lessened by putting the muscles at complete rest, with the foot in an improved position, in a plaster-of-Paris dressing for several weeks. This measure is recommended at the beginning of treatment in all cases of pronounced severity.

Tenotomies.-These will, of course, remove immediately all influence on the joints of the spasmodic muscles; but after the tendons have united their influence will again be exerted. Before this union takes place, much may, however, be gained by massage and exercise of the other muscles. Furthermore, the rest obtained by the nervous system after tenotomy is beneficial to the general condition. As a rule operation is not advisable immediately after a case comes under treatment. If braces can be made to overcome the spasmodic muscles, tenotomies can be advantageously postponed for some months or even years; especially as, in many of these cases, there comes a time when the spasms diminish in intensity, contractures form, and the result of a tenotomy will then be more enduring. After tenotomy of these muscles, if the spasm is very pronounced, the proximal end is drawn some distance from the distal end and therefore it is well to put the foot up at a greater angle than 90 degrees, so as to assure a firm union.

Neurotomy.-The reports of many observers that after simple tenotomy of the tendo Achillis in these cases, the spasms in all the muscles of the extremity frequently lessened, and even the mental condition improved, led me to perform intraperineural neurotomy 
so as to give a more widely distributed and a more prolonged rest to the muscles. The results in two cases operated were very encouraging and were reported in the American Orthopedic Journal of November, I909. In both of these the sciatic was temporarily paralyzed. More recently I divided and sutured the posterior tibial nerve and the branch from the internal popliteal to the soleus. This left the nerve supply to the skin unimpaired and did not affect the muscles supplied by the external popliteal. Thus the stretched and weakened dorsal-flexors and abductors could be strengthened and shortened by exercise and massage. As the supply to the gastrocnemius was uninterrupted, the tendo Achillis was divided. The result in this case was excellent. After the tendo Achillis had united, the patient could walk with the foot in the normal position.

Injections of Alcohol.- These have been made into the nerve by Schwab and Allison. However, an operation is necessary to expose the nerve to be injected and the paralysis from nerve-block is not, in my opinion, of sufficiently long duration to warrant the procedure. They have given many reports of their cases and they themselves are greatly encouraged by their results.

Stoeffel's Technique.- Stoeffel * lessens the aggregate energy of a spastic muscle by reducing the number of its active parts. This he does by severing and resecting the motor nerve funiculi of these parts. His

* "The Treatment of Spastic Contractures," Adolf Stoeffel, Mannheim. The American Journal of Orthopedic Surgery. May, I9i3. 
object is to reduce the energy of the spastic muscle, leaving only power enough for it to perform its work as it would under normal conditions. In a severe case of spastic pes equinus he resects two-thirds of the nerve branches to the gastrocnemius and half of the supply to the soleus. In slight cases, one-third of the nerve branches to the gostrocnemius and half the supply to the soleus. In both cases he destroys the nerves to the digital flexors. In about one hundred of these nerve operations in various conditions and localities, he has never experienced a lesion of the nerve tract that was not intended. It will be at once apprehended that a remarkable knowledge of anatomy, experience in dissecting and skill in operating are demanded. Stoeffel himself warns against this operation being undertaken unless the surgeon has fitted himself for this work. 


\section{INFANTILE PARALYSIS}

Residual Paralysis. - The residual paralysis after an attack of anterior poliomyelitis, more often affects the muscles controlling the foot than any other group, and of this group the tibialis anticus is the one most frequently left in a paralyzed condition. Not only is it uncertain as to the exact limit of time after an attack of this disease, when improvement may be expected from resuscitation of the cells in the anterior cornua, probably six months and perhaps longer; but paralysis may remain, after the nerve cells have entirely recovered, from stretching, atrophy, and degeneration of the muscles. Therefore it is essential that before any operation is undertaken the existing cause of the paralysis be unquestionably ascertained. Theoretically electricity offers a means of determining whether the nerve-cell or the muscular structure is at fault and it is certainly of great value in the hands of an expert. To the ordinary surgeon, however, especially when the patient is a child, the technique is too difficult of application to be entirely satisfactory. The best way to satisfy oneself that the paralysis is permanent is to treat the doubtful muscles for a prolonged period as though a return of power was 
expected. Even when contractures have not formed, gravity, or use in a deformed position, may stretch and prevent the return of power to muscles histologically
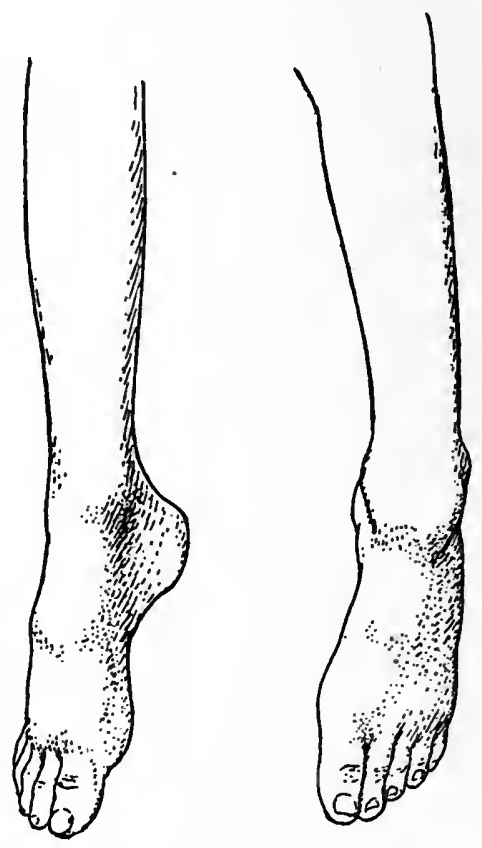

Fig. 6I. DANGLe- $\dot{F}$ OOT

From photograph of child with complete paralysis of the lower extremities. The child is seated on the end of a table with the feet dangling. Gravity has placed the feet in the position of equinus and slight inversion; the position directed by the shape of the articulations, uninfluenced by muscles.

intact and sound. For instance, an equinus position retained at a flail ankle on account of gravity, may be sufficient to continue a paralysis of the dorsal flexors after their cornual cells have quite recovered. 


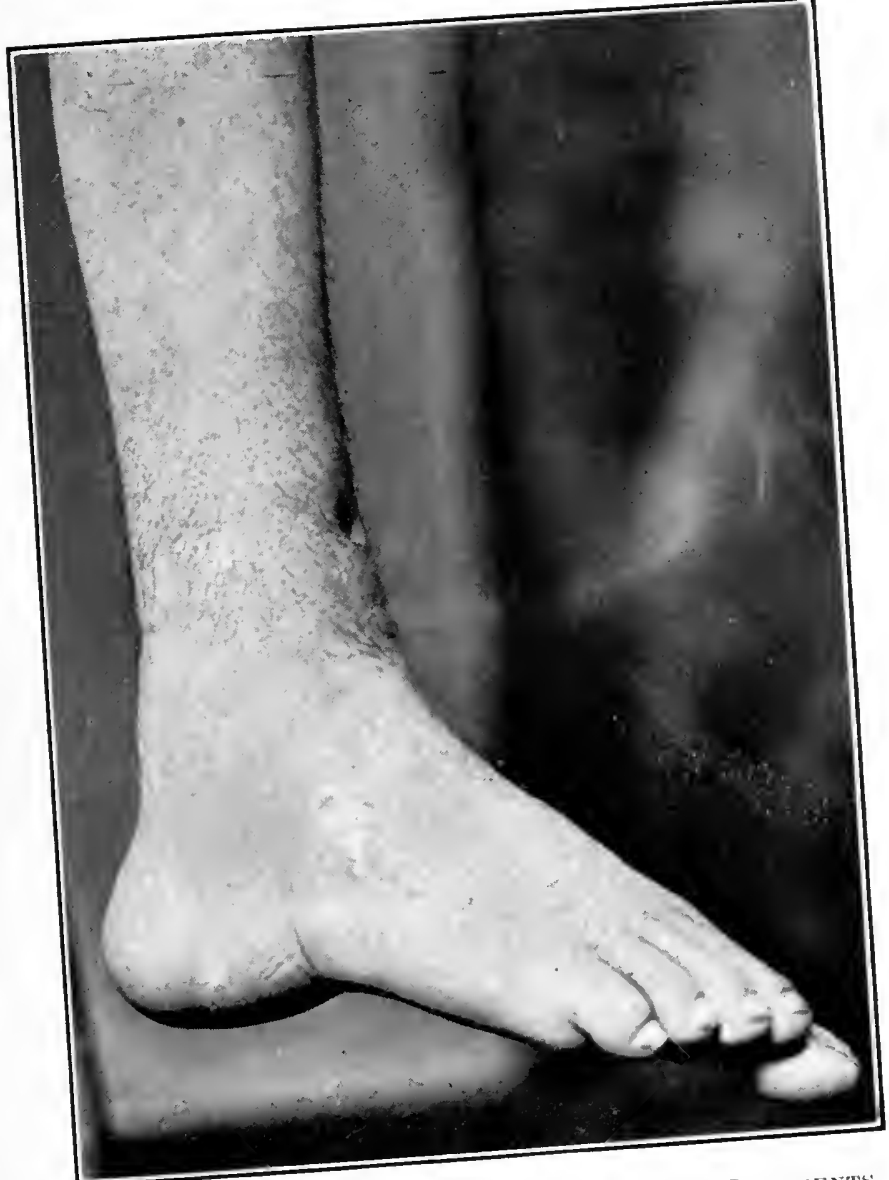

Plate III.-Frail-ANkLE Supponted BY Silk Liganests

passing over the ankle were paralyzed. The muscles passis deformity due to contractures of the foot presented a Silk ligaments were inserted an months after plantar tissues. Sill they supported the root ten monging free. graph shows how well the sed on a table, 

Diagnosis.- By placing a finger on a tendon, a contraction may be felt which is too weak to be demonstrated by any visual movement. Even the slightest. voluntary contraction of a muscle is proof that its nervous mechanism is intact, at least in part, and therefore the prognosis for that muscle is good. The degeneration of muscle fibers may be extensive, yet diligent attention to the details of treatment, over a prolonged period, will often be rewarded. Muscles have regained their functions after years of disuse.

These remarks are especially of weight when the question of an operation arises which would not be necessary should one or more of the inactive muscles prove to be sound. As to arthrodesis, it should not be performed in any case on the child with growing bones. The interference with the length of the leg will not be so great after arthrodesis of the ankle as after that operation on the knee joint, but postponement of this operation on the child is, as a rule, to be made.

Paralysis of the foot from infantile paralysis will be taken up under three divisions; recent cases, untreated cases of long standing, and cases of undoubted permanent paralysis.

\section{RECENT CASES}

We shall not concern ourselves with the general treatment but confine ourselves to the foot. The primary object is to preserve the healthfulness of all the structures: bones, ligaments, and muscles. While strain and 
stretching are prevented, normal function should be exercised in so far as possible. Whatever will assist in obtaining these ends must be accepted as valuable.

Apparatus.- Of whatever material it is made, the object of an apparatus is to prevent the strain of the muscles and ligaments from the action of the sound muscles, from gravity, and from weight-bearing in a faulty position and simultaneously permit as much functionating as possible.

Active Movements.-When all the muscles are not paralyzed, those which have escaped must be exercised or suffer atrophy. Exercise is given by removing the apparatus and by having the sound muscles voluntarily contracted against the resistance of the nurse's hand. For instance, if the calf muscles are paralyzed, the patient is told to draw the foot up, in dorsal flexion, while the hand of the nurse resists the movement sufficiently to increase the work done and at the same time prevents hyperflexion. Thus the anterior muscles are exercised while over-stretching of the calf muscles and of the posterior ligament of the ankle-joint is prevented.

Voluntary contraction of a muscle is far superior to massage or to contraction from an external stimulant, such as electricity. Therefore attempts should be made to contract the paralyzed muscles voluntarily. In the above exercises for the preservation of the anterior muscles, in order to keep diligently attempting to contract the calf muscles, the child should be told to try to extend the foot when the nurse brings the foot into extension. 
If the movements are done rhythmically it is much better than spasmodically; and if but one foot is paralyzed, the other should voluntarily be put through the same movements synchronously with the one being exercised. It will be found to be of assistance if the commands are given in a sing-song fashion, as: "up!" "down!" "up!" "down!"

What may be accomplished by these exercises when they are done conscientiously, three times a day, for weeks and perhaps months, seems little short of miraculous. The patience, time, and trouble are amply rewarded if voluntary power does return; for no transplanted muscle can equal the restored normal muscle.

Massage.-This is especially indicated for the paralyzed muscles. It is of undoubted value in preserving the muscle tissue. Degeneration and atrophy are at least delayed. Not only are the muscles benefited but also the skin, blood vessels, ligaments, and even the bones. The therapeutic value of massage is principally from the increased flow of blood and lymph which it causes; the carrying away of effete material, and the supplying of material necessary to the maintenance of health. The stimulation of nerves not paralyzed, the sensory nerves and the nerves to other structures than the paralyzed muscles, is probably of value also. Therefore massage should be deep and with long, gentle strokes, so as to empty the deeper vessels; and not a simple rubbing of the skin. It is very important, however, that it be borne constantly in mind that paralyzed muscles are 
weak, they can not stand rough handling, and massage such as kneading may do more harm than good.

Electricity. - This is of value as a stimulant to contraction of muscle fiber. If the stimulation is carried to the muscle fibers through a nerve, it may help to preserve the health of the nerve as well, but on this point I know of no physiological data from the laboratory. As has already been stated, voluntary contraction is the best preservation of the health of a muscle, but where this is impossible, on account of the paralysis, the muscle fiber contraction from an external stimulus is the next best measure. Usually the galvanic current will be necessary; but as soon as the nervous mechanism begins to be reëstablished, the faradic current may be used. Two or three contractions are all that should be made at any one séance. More will exhaust the fibers and hasten atrophy. Electricity is probably of no value except where it causes contraction of muscle fiber.

Heat. - The temperature of the paralyzed parts is lowered, not only as a result of the paralysis of the nerves supplying the vessel-walls and the consequently sluggish blood stream; but as a result of the inactivity of the various structures, which slows the blood and thereby allows greater dissipation of heat before it is renewed. It is of the greatest importance that the normal temperature be maintained as nearly as possible. This may be accomplished to a great extent by preventing conduction through unconfined air. Extra clothing, as two pairs of stockings, should be worn. What- 
ever means are taken, the surgeon should satisfy himself that the object is being attained and should bandage the foot and leg in cotton-wool should it become necessary. External heat may be applied with benefit just before each séance of exercises, massage, and electricity, such as the heat from an open fire or the application of warm clothes; but the extra clothing should be sufficient for the rest of the time.

Functional Use.-This is the best therapeutic measure for obtaining normal growth. It must be limited, as any apparatus efficient in protecting the paralyzed muscles and the weakened ligaments from strain, must limit exceedingly, if it does not entirely prevent, the use of the unaffected muscles. The function of weight-bearing is of inestimable value in stimulating the growth of the bones and indirectly in increasing the temperature of the foot. It should therefore, be provided for and encouraged in every instance.

\section{UNTREATED CASES OF LONG STANDING}

The fact that this class has had no treatment, or only treatment of a negligible quality or quantity, or both, raises a question which is all important in our prognosis: Are the apparently paralyzed muscles beyond the possibility of repair? The electrical reaction will tell much, but personally I prefer, whenever possible, to begin treatment with the aim of giving each muscle an opportunity to reassert itself. Therefore operation should, I feel, be limited to the replacing of the struc- 
tures of the foot in their normal relations and this should be followed by the treatment laid down for recent cases.

In these cases we have either the flail joint of the ankle with more or less deformity of the foot resulting from paralysis of all the muscles, and from the foot's

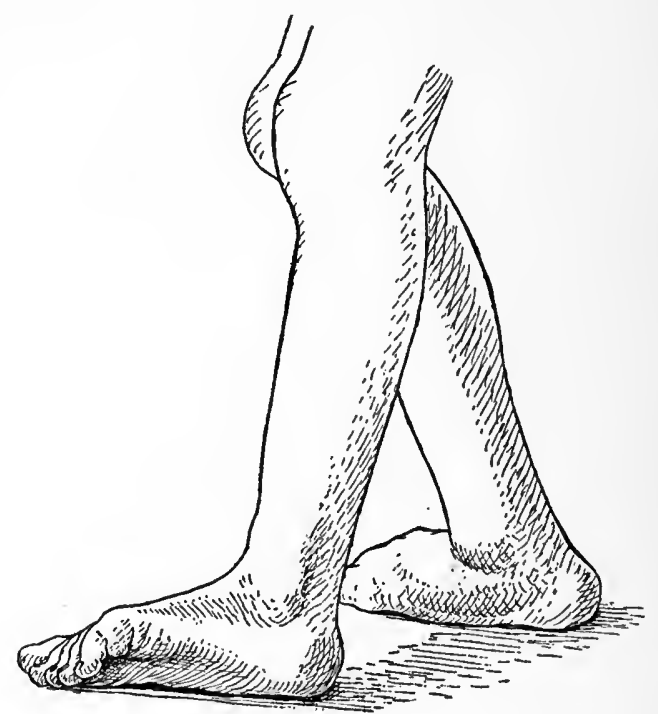

Fig. 62. Paralytic Talipes Valgus

The only muscular power was expressed by a slight ability to flex the smaller toes. Astragalectomies were performed with marked improvement.

being used while in this condition; or we have deformities resulting from shortening of healthy structures, muscles and ligaments, from stretching of unused muscles and of unprotected ligaments, and from changes in some of the bones, resulting from their being used to bear weight and aid in progression while retained in one po- 
sition or at least while not enjoying their normal range of motion.

Some of the acquired deformities following paralysis are :

Valgus.- This is the most common and accompanies paralysis of the tibialis anticus or of both the anticus and posticus. Sometimes it is associated with a re-

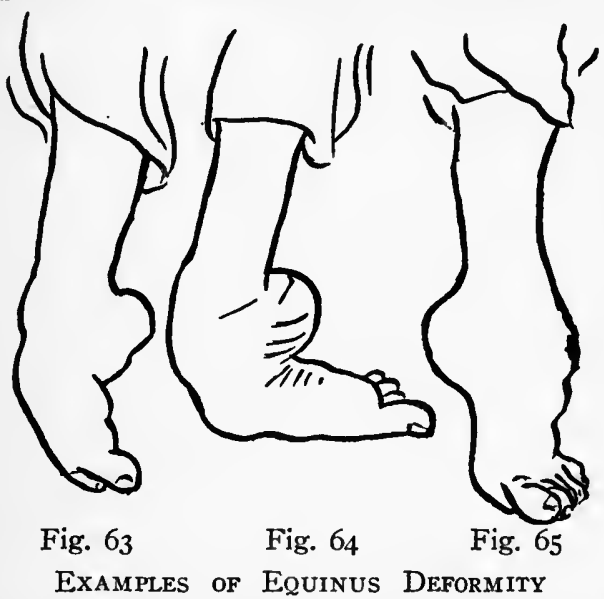

If Fig. 63 is used in standing and walking, it may progress to the deformity seen in Fig. 64 . Fig. 65 becomes more confirmed in the deformed position without the contour of the foot becoming much altered.

sidual weakness of the other anterior tibial muscles, in which case there is usually a shortening of the tendo Achillis and an equino-valgus rather than a pure valgus.

Varus.-This is rather uncommon. It results from paralysis of the peroneals. The deformity does not become extreme and is not of so much consequence as valgus. 
Equinus.-This results from paralysis of the anterior tibial muscles. Dorsal flexion being lost, the extensors can not be fully drawn out except by passive flexion of the tibia in walking with the foot held straight ahead or turned slightly inwards. With the foot so held, when it is raised at the end of the step to advance it for the next step, the toe drops to full extension, or rather to hyperextension, and by dragging makes walking difficult. To avoid this embarrassment, the foot is turned outward and full dorsal flexion no longer takes place. Thus full extension of the posterior muscles, the drawing of them out to their full length, never occurs and they consequently become permanently shortened. While a patient with paralysis of the anterior muscles is lying down or sitting with the foot hanging, extreme plantar flexion is present. The anterior muscles and ligaments become more and more stretched until the equinus is marked.

Whether valgus or varus co-exists with this deformity will depend to a great extent on whether the unprotected foot is used in walking soon after the attack of the disease. If the influence of walking is a minor factor, the foot will become more strongly fixed in the position which it assumes when hanging free, and equinovarus results; on the other hand, if walking is undertaken early and persisted in, equino-valgus will be found. The former deformity, however, is much more usual for the following reasons: the plantar muscles, not having the anterior muscles to extend them, suffer contraction 
(unless frequently and strongly stretched by the foot's being used in a valgus position); and by their flexion of the medio-tarsal joint they raise the dome, adduct the forefoot, and help to produce varus. With this position of equino-varus, the toes will be extended at their first interphalangeal joints, because of the contraction of the interossei, which are inserted into the extensor tendons over the first phalanx. The distal phalanges of the smaller

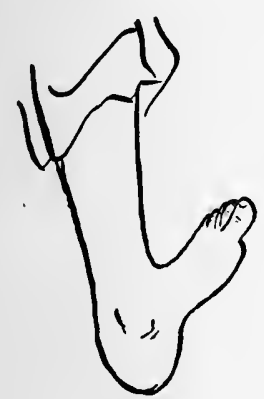

Fig. 66

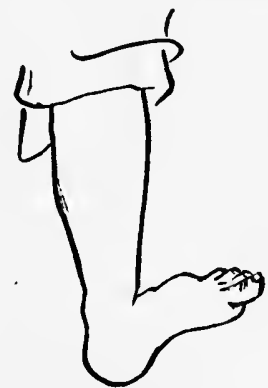

Fig. 67

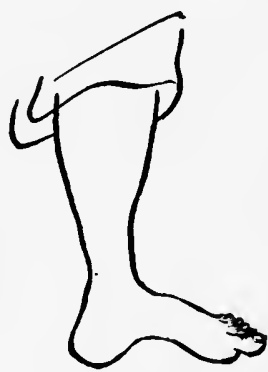

Fig. 68

\section{Varieties of Calcaneus}

In Fig. 66 the calcaneum is rotated on a transverse axis so that the posterior surface of its tuberosity is walked upon. In Fig. 67 the deformity is less marked, and in Fig. 68 the forefoot is flexed and a cavus produced by the long flexors being intact.

toes will be flexed by the shortening of the long flexor. The contracted plantar tissues may cause a more marked cavus condition than a varus. This cavus, however, is found to be due entirely to flexion of the forefoot. If the forefoot can be forced into its normal position the cavus disappears.

Calcaneus.- With a paralysis of the gastrocnemius and the soleus, the force of contraction of the 
plantar muscles attached to the os calcis is felt entirely in that bone, unprotected by the tendo Achillis. Added to the pull of the plantar muscles, is that of the anterior muscles, especially of the long extensors. This force upon the os calcis tends to rotate it on a transverse axis and this rotation is further increased by walking, for the

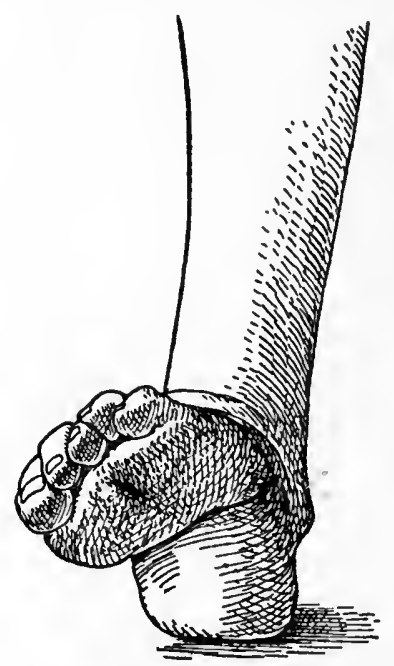

Fig. 69. Paralytic Calcaneus

Showing the transverse plantar groove opposite the medio-tarsal joint caused by the folding together of the forefoot and hind-foot.

impact of each step is received more and more upon the posterior surface of that bone, as the posterior extremity of that bone and the heads of the metatarsals are more and more approximated. Calcaneus may be accompanied by very marked cavus or folding of the forefoot so that the foot of a Chinese lady is more or less re- 
sembled. It is a matter under dispute whether or not calcaneus must be the sequence of a primary dorsal flexion, which, in the severer grades, has, as a consequence of walking and of gravity, given way to a dropping down of the forefoot, helped sometimes by an intact peroneus longus.

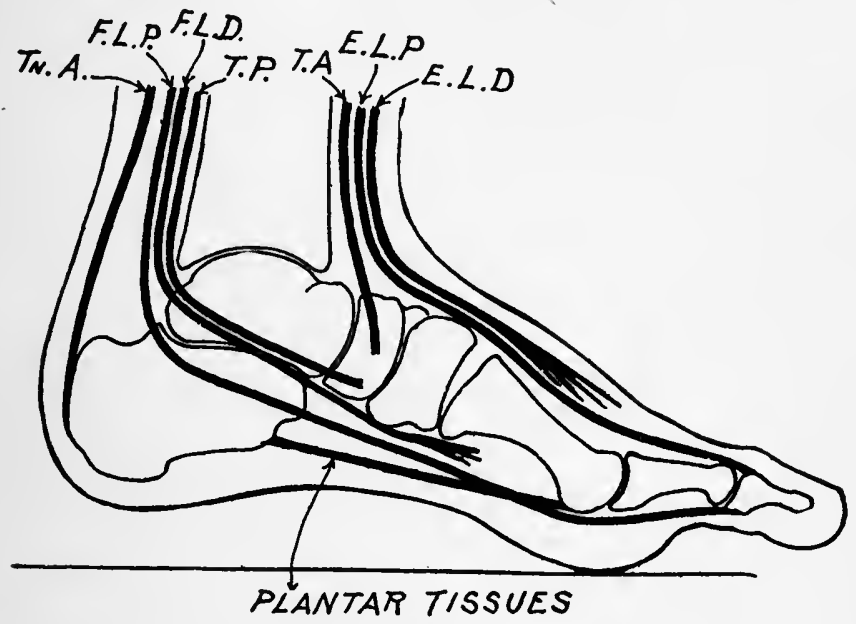

Fig. 70. Talipes Plantaris

The anterior muscles are weak and the plantar-flexors of the forefoot have raised the dome. The ball of the great toe is on a lower level than the heel.

Cavus.- Aside from the condition of cavus which may accompany other paralytic deformities, there is one which is widely recognized; and although it is probably a result of other than infantile paralysis, being most common after the age of childhood, we shall take this opportunity to describe it. 
Hollow Claw-foot.- The arch is increased and the toes are in a position of dorsal-flexion at their metatarsalphalangeal joints and of plantar-flexion at their first interphalangeal joints. Duchenne attributed it to a paralysis of the interossei and lumbricales, which plantarflex the first phalanges on the metatarsals and dorsalflex at the first interphalangeal joints; and to a paralysis

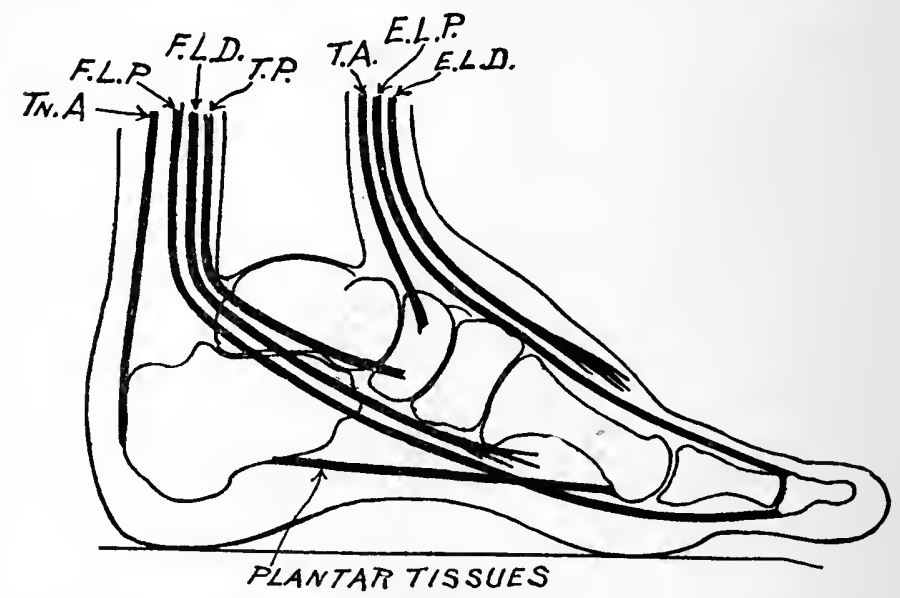

Fig. 7I. TAlipes ARcuatus

The anterior muscles are weak and the dome is raised, but the forefoot can be dorsal-flexed to the level of the heel.

of the short flexors and of the abductor of the great toe. Thus contraction of the long flexors and extensors produces the clawed-toes. Golding-Bird, however, attributed the condition to paralysis of the peronei; the unresisted action of the adductors causing the hollow-foot, and the clawed-toes resulting from the ineffectuality of the action of the extensors on account of the fact that the 
proximal ends of the first phalanges are held down by the contracted plantar fascia. As this deformity is usually associated with a right-angled contraction of the tendo Achillis and as we have shown how this contraction may cause a hollow-foot, we are inclined to believe that the shortened heel-cord may be a causative factor.

In untreated cases of long standing those muscles which show no definite improvement after three or four months of treatment, may be condemned as permanently paralyzed.

CASES OF UNDOUBTABLE PERMANENT PARALYSIS

All the muscles, ligaments, and fascix which have been retained in one position for a protracted period, will have accommodated themselves to that position. If their points of attachment have been held nearer together than normally they will be contracted so that separation of the points of origin and insertion in their usual extent will be impossible without forcible stretching or division of the contracted tissues. The points of attachment of these tissues have been brought nearer together by forces, either intrinsic or extrinsic, by either muscular pull or external pressure. An illustration of the former in causing contractures is seen in the equinus produced when the anterior muscles of the leg are paralyzed and the action of the posterior muscles is unopposed. Voluntary power of reducing the equinus position is lost and as the calf muscles are not stretched to the length they have in dorsal flexion they become contracted, to- 
gether with all the other soft structures which are held in a shortened position.

Furthermore, the plantar muscles will hold the forefoot flexed at the medio-tarsal joint since they are unopposed by the anterior muscles, and a cavity deformity

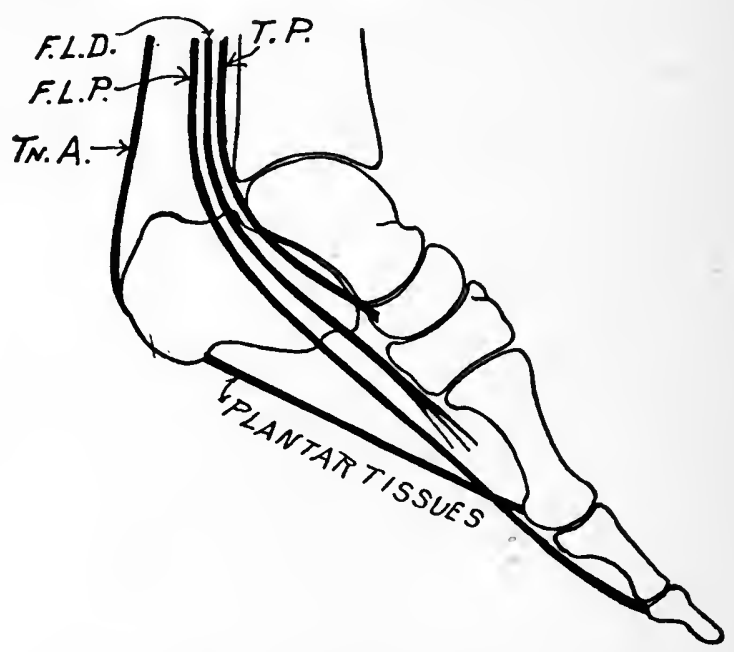

Fig. 72. Paralysis of the Anterior Muscles

The foot is drawn into the position of equinus. The dome is raised, owing to the unresisted action of the plantar-flexors of the forefoot and contraction of the plantar fascia. The toes are not more flexed because the interossei, inserted into the tendons of the long extensors, are intact. Walking also helps to keep the toes extended.

will be added to the equinus. There will also be flexion deformity at the metatarsal-phalangeal joints; but the flexion deformity will not have taken place at the interphalangeal joints because the interossei muscles, through their attachments to the long extensor tendons over the 
first phalanges, will keep intact some power of extension at these joints.

Whether an external force, as weight-bearing, increases or decreases a deformity of the foot must depend on its direction, strength, and duration; so the amount of cavus deformity and of deformity of the toes in a

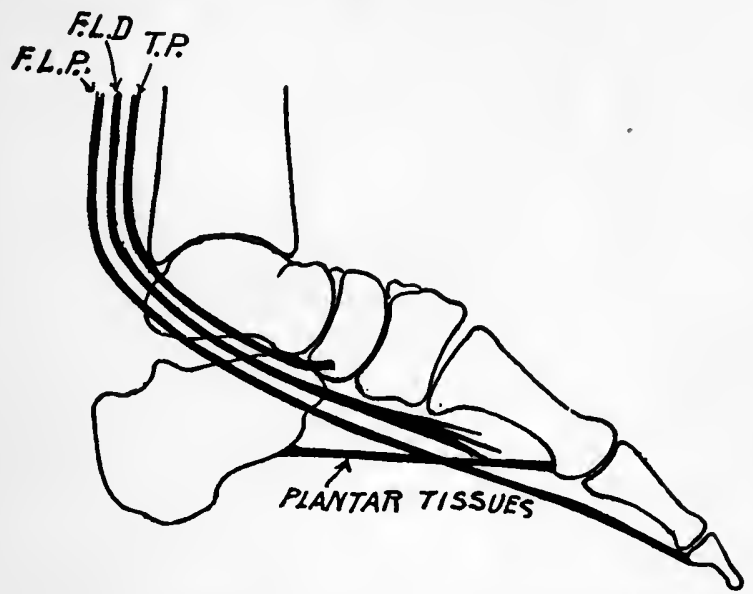

Fig. 73. Paralysis of the Tendo Achillis and the ANTERIOR MUSCLES

The sound, long flexors flex the forefoot, contraction of the plantar tissues approximate the calcaneum and the metatarsals; thus a cavus is produced.

case with the above mentioned paralysis, will be influenced by the amount and manner of the use to which the foot is put.

If all the muscles passing over the ankle are paralyzed and only the plantar muscles are left intact, there will be added a rotation of the os calcis on a transverse axis, so that its tuberosity will be moved downward and for- 
ward, and its anterior extremity upward, and, at first, forward, and then backward. This is brought about through the absence of the calf-muscles' offering a point of resistance by holding the os calcis firm, when the plantar muscles contract; and consequently at each contraction of these plantar muscles the force is not firmly resisted by the os calcis and the bone gives $\circ{ }^{\circ}$ the di-

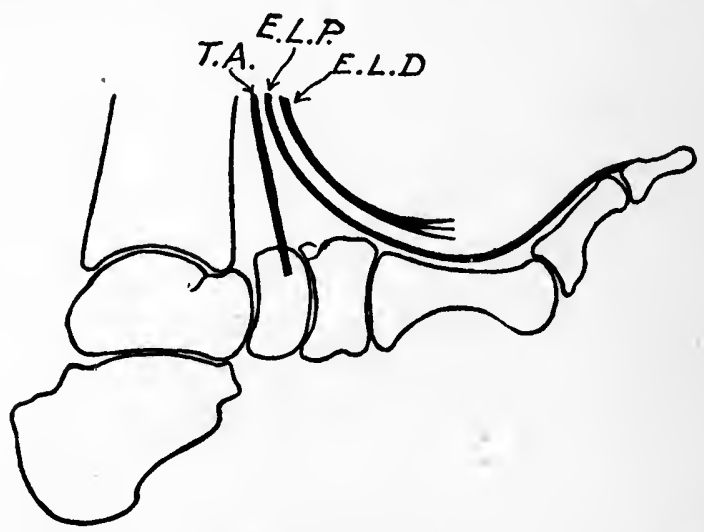

Fig. 74. Paralysis of the Posterior and the Plantar Muscles

The foot is drawn into the position of dorsal flexion. In walking the heel is presented to the ground in advance of the toes and as the impact of the calcaneum is received more and more, as deformity progresses, on the posterior tuberosity of that bone, a calcaneous results.

rection of this rotation. Thus a calcaneus is produced which, added to the flexion of the forefoot, forms a marked cavus or hollow-foot. With this flail ankle, the foot, in walking, will take a position of eversion; and if much used will tend more and more to flatten the dome, but this force will not prevent, although it may delay, the calcaneo-cavus. 
If the posterior muscles are alone paralyzed, the os calcis will suffer the rotation as described above and the forefoot will be drawn up into exaggerated dorsal flexion. Flexion will not only be pronounced at the ankle joint, but there will be extension at the medio-tarsal joint, and the patient will walk upon the heel.

If all. the posterior muscles are paralyzed, and the

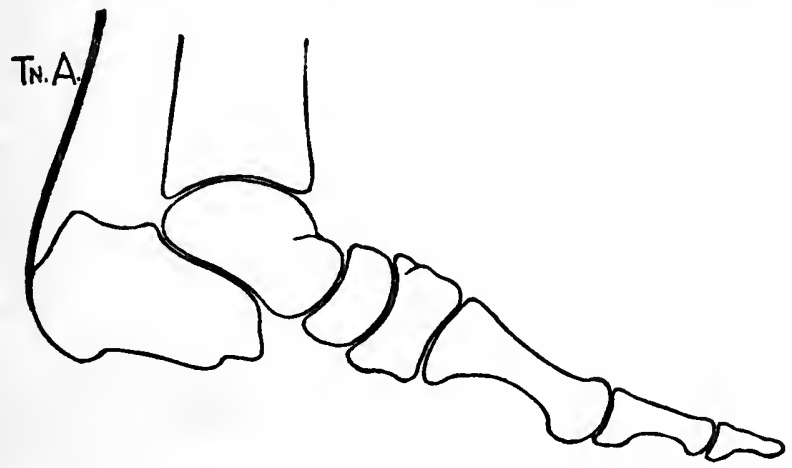

Fig. 75. Paralysis of All the Muscles Except the GastrocNEMIUS AND THE SoleUs

With the tendo Achillis normal the heel is raised, but an equinus of the foot is not produced because there are no muscles to flex the forefoot and because use of such a foot tends to lower the dome, to extend the medio-tarsal joint.

plantar muscles as well, the os calcis will not be rotated and the toes will be held in extension. There will be dorsal flexion at the medio-tarsal joint and the dome will be flattened.

When the tibialis anticus alone is paralyzed, the foot will be drawn into eversion whenever dorsal flexion takes place; and in standing, the inner border of the foot will fall into a position of valgus, especially marked if 
there is paralysis of the tibialis posticus accompanying it.

\section{TREATMENT}

Reduction of Deformities. - Whether our object is to discharge the patient wearing braces or whether we intend to attempt the restoration of power in the control of joints by muscle transplantation, or to support them by the use of artificial ligaments or by arthrodesis we plan to immobilize them, the first concern of the surgeon must be the reduction of deformities. Reduction of deformity may be undertaken by manual or instrumental force, or by open or subcutaneous cutting operations. Most contractures about the foot can be made to yield to stretching, without the use of an anesthetic, if the forces are correctly and repeatedly applied and the improvement constantly maintained. To be painless and at the same time efficient, the forces must be applied with such exactitude and perfect control that special apparatus is generally necessary.

Instrumental Stretching.-In the hands of the orthopedic surgeon, Shaffer's antero-posterior and lateral traction shoes will give most satisfactory results. Special mechanical knowledge and an abundance of patience, together with the absolute confidence and coöperation of the patient, are necessary; and therefore these apparatuses have never been very popular with the general surgeon. A keen knowledge of mechanics is absolutely essential for the successful use of orthopedic apparatus. 
Without this knowledge the surgeon had better content himself with the knife and plaster-of-Paris. Therefore, I am persuaded that it is advisable for me to give but a short description of the lateral-traction-shoe and that is only for those who are or might become orthopedic surgeons.

Lateral-Traction Shoe.- The lateral-traction apparatus is based upon the three varieties of rotation on the three axes of movements of the foot. It consists of a calf band, $A$, to which is attached a single upright, $\mathrm{A}^{\prime}$. This upright is always placed on the side toward which the deformity looks; in varus on the inside, in valgus on the outside of the leg, the instrument being a pusher. At a point just above the axis of motion of the anklejoint, are placed a lateral joint, $\mathrm{C}$, which is acted upon by a hinged lever and screw; and $\mathrm{O}$, an arm, which pushes the lower part of the apparatus toward the deformity to any desired extent. The distal end of this arm is free, and, as the arm is turned away from the deformity by key $\mathrm{B}^{\prime}$, the foot part of the apparatus takes any lateral position required. This lateral hinge, with its lever and screw, is intended to meet especially the inversion of the os calcis. When traction is applied, it antagonizes the tibialis posticus muscle contraction as well as the shortening of the internal lateral ligament. Just below the hinged lever and screw is an antero-posterior joint, E, a worm and screw controlled by the key $\mathrm{E}^{\prime}$. With this any degree of flexion and extension may be obtained. Still lower and acting upon the an- 
terior part of the foot-plate (which is divided transversely at a point corresponding to the medio-tarsal joint) is the traction rod placed upon the inner side of the apparatus and moving in the cylinder F. It is con-

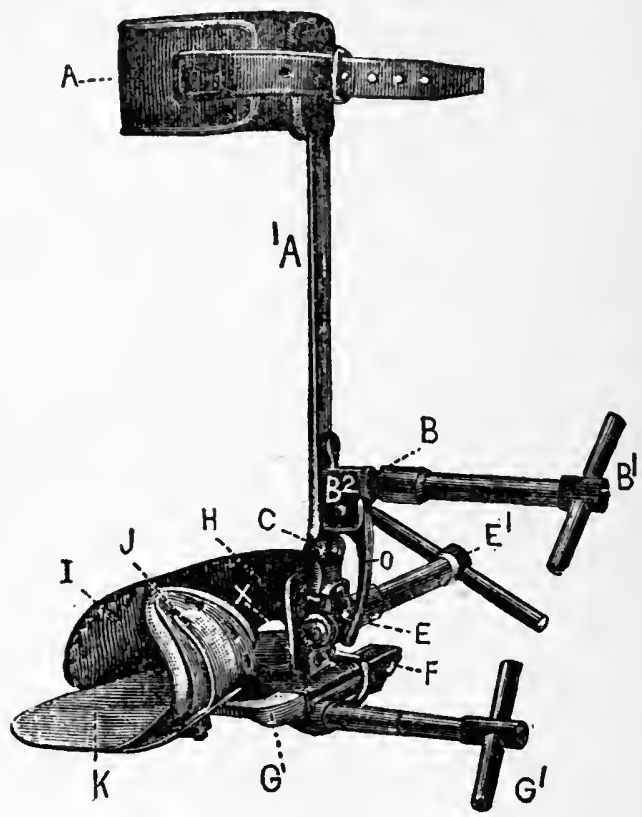

Fig. 76. Shaffer's Lateral Traction Shoe

The movements about the three chief axes of the foot are controlled by the three keys. A full description is given in the text.

trolled by the key $\mathrm{G}^{\prime} . \mathrm{H}$ is the heel-cup, through the openings in which, $\mathrm{X}$, are passed the ends of the astragalar strap. The outer part of the heel-plate is extended quite well forward to form a resistance to the rotating movement imparted to the foot by the traction 
rod G. The inner border of the foot-plate is curved over the dorsum of the foot, $\mathrm{J}$, in order to grasp it as it moves from the center of motion at the outer border of the foot. The heel-plate part of the heel-cup has the semicircular opening to permit the descent of the heel when traction is applied, the same as in the antero-posterior shoe.

Determination of Obstruction.-It is worthy of remark, that as exact a determination as is possible, as to what tissues are obstructing correction, should be made. The X-ray will assist in demonstrating the extent of any bony obstruction, but the hands of the surgeon are of the first importance. The resistance caused by bone is of a decidedly different "feel " from that due to ligament or muscle. It must be borne in mind that the movement allowed by the removal of one obstruction may be immediately blocked by another tissue which is shortened or otherwise abnormal. Thus an operator may be greatly disappointed at finding that a simple tenotomy, while sufficient to remove all immediate obstruction to correction, has simply served to bring into action other obstructions.

It is worth while to spend much time and effort in trying to lengthen soft tissues by force, applied with the hand or wrench; but bony deformities will yield only to constant pressure and friction, and to the chisel.

Recovery of Muscles.- After the restoration of the foot to its normal position, the bones being in their normal relations to each other, it should be maintained thus 
for a sufficient length of time to assure the surgeon that all possible recovery of muscular power has taken place. Even though a muscle has been out of use for some years, it may yet enjoy a return of power if the opportunity is given. That is to say, although the cells in the anterior horns, which supply the muscle, were attacked by the original disease, they may have recovered but are unable to cause contraction of the muscle because the muscle is so stretched. It is impossible to say in any given case, how long a time should be given to Nature to show a return of muscular power. As a rule, if a muscle is kept constantly in the best position for it to contract for five or six months, and if at the end of that time there is no apparent return of nervous control, it may be condemned as permanently paralyzed.

Braces.-During this period of giving Nature an opportunity, braces should be applied. The design of

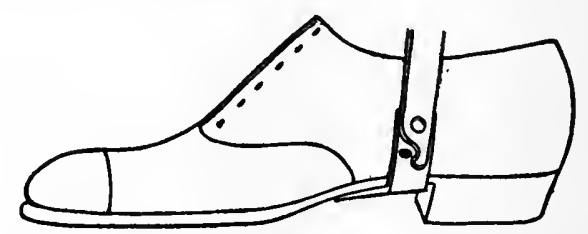

Fig. 77. Right-Angle Stop at Ankle-Joint

With this stop plantar flexion beyond a right angle is prevented. It is to be used in paralysis of the dorsal-flexors.

the brace should be such as to protect the paralyzed muscles from being stretched by the sound muscles. If the anterior muscles are paralyzed the brace must be so constructed as to prevent plantar flexion beyond a right 
angle. If the posterior muscles are paralyzed, dorsal flexion must be prevented beyond a right angle. The easiest way to make the joint of the brace meet these requirements, is to have a tongue extend beyond the joint with a peg to stop motion in the direction of the healthy muscles.

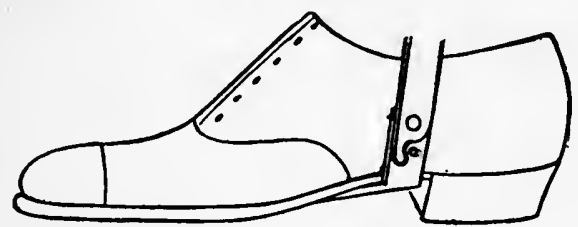

Fig. 78. Reverse-Stop at ANKLE-Joint

With this stop dorsal-flexion is limited to a right angle with the leg. It is indicated in paralysis of the plantar-flexors.

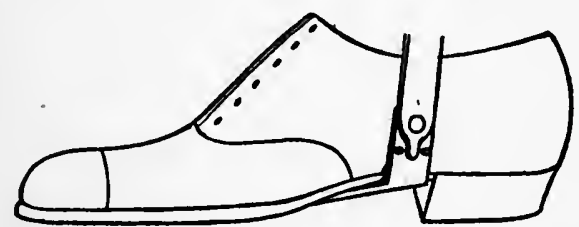

Fig. 79. A Stop-Joint Allowing a Few Degrees of Motion Only

This is of use when extensive paralysis is present. It facilitates walking by permitting the body to be brought over the foot while it is flat upon the ground. If there is no movement at this joint, the heel must be raised as soon as the vertical is reached, which markedly embarrasses the walk.

The brace may be secured to the outside of the shoe or it may be made with a foot-piece and be worn inside of the shoe. It will be found easier to fit the brace if it is made to go outside of the shoe and though that shoe will always have to be worn, unless other braces are made and fitted to other shoes, we should advise that 
the surgeon of little experience in such matters use the outside brace. The inside brace, if not accurately fitted, is likely to be uncomfortable; and many patients have discontinued treatment, when the principle of the brace was perfectly correct, because they despaired of ever being able to obtain an accurate fit.

The joint of the brace must not be too high; this fault

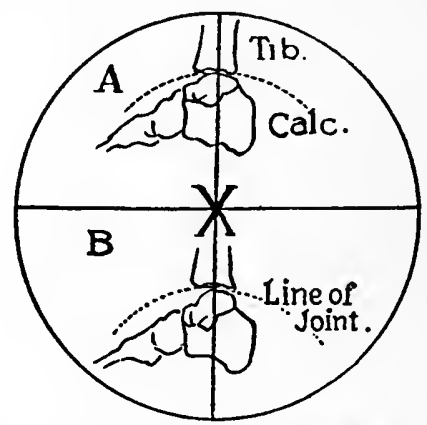

Fig. 80. Location of AnkLe-Brace Joint

Illustrating the necessity of the joint of a brace being below the ankle-joint: $X$ represents the joint of the brace, if within the arc of movement at the ankle as in 'A, no interarticular pressure will be caused by motion, while if the joint of the brace is above the ankle-joint, as in B, motion in either dorsal or plantar flexion must cause interarticular pressure.

is very frequently made by those unfamiliar with physiological mechanics. The center of the joint should be opposite the tip of the external malleolus, or even a little lower. The side bars should lie close to the leg and nut stand away a quarter of an inch or so, and this fit should be just as accurate over the malleoli. If valgus is assumed upon weight-bearing, a pad should be placed on the outer side, low down, so as to hold the tarsal bones 
in their proper position. If varus is assumed under these circumstances, the pad should be placed on the inner side. The pad is not to push over the ankle, the prominent malleolus, as some seem to suppose.

Wedge Sole.-When the paralysis is very slight and confined to one or two muscles, as the tibialis anticus or the peronei, it may be necessary only to apply a wedge to the shoe, such as was described under congenital clubfoot; or a steel insole can sometimes be fitted to meet all requirements.

When one is satisfied that the remaining paralysis is permanent, consideration may be given to the possibility of some operation relieving the patient from the necessity of wearing a brace for the rest of his life.

Tendon Transplantation.- This is very successful if correctly planned and properly executed. The planning must provide for the substitution of sufficient new muscular force to take the place of the paralyzed muscles, without too great weakening of movements in other directions; and the execution of the operation must be done with the most careful transposition of the tendon, providing as direct a line as possible to the new insertion, arranging the bed for the tendon so that it may move freely and not be held by adhesions, and exhibiting a perfect aseptic technique. The after treatment is of an importance second only to the operation itself : immobilization for four weeks followed by gentle massage and active and passive movements for several months.

The condition in which the tibialis anticus is the only 
muscle paralyzed, is the most promising for this operation. The muscle to be transplanted is the extensor longus pollicis. Three very small incisions are all that are required, though one long curved incision may be used. The tendon of the long flexor is divided over the middle of the metatarsal, the distal end secured by silk or linen suture to the tendon of the flexor longus digitorum going to the second toe, and the proximal end drawn out through an incision made over the ankle at the forward turn made by the tendon as it goes forward to the great toe. An incision is then made over the tuberosity of the scaphoid, a tunnel made to the incision above, and the tendon drawn down through this and sutured with silk to the periosteum or drawn through a hole drilled in the bone, and then sutured. The tendon should be drawn taut before it is sutured, with the foot in dorsal flexion to eighty-five deyrees and in slight inversion. The position of the foot is retained in a plaster-of-Paris dressing for four weeks. A brace is then worn for six months to prevent the extremes of motion. A night shoe is also prescribed, and massage given daily. It may be necessary for the patient to wear a wedge on the inner side of the sole after the brace is discarded.

This operation may sometimes be supplemented, when eversion is pronounced, by also transplanting a slip from the extensor longus digitorum to the scaphoid. If this is done the distal extremity of the extensor longus digitorum is secured to the periosteum of the metatarsal. The tendon of the peroneus longus may also be used to 
reënforce the flexor longus pollicis. This is done by dividing it over the head of the os calcis, drawing it out through an incision two inches above the tip of the ex-

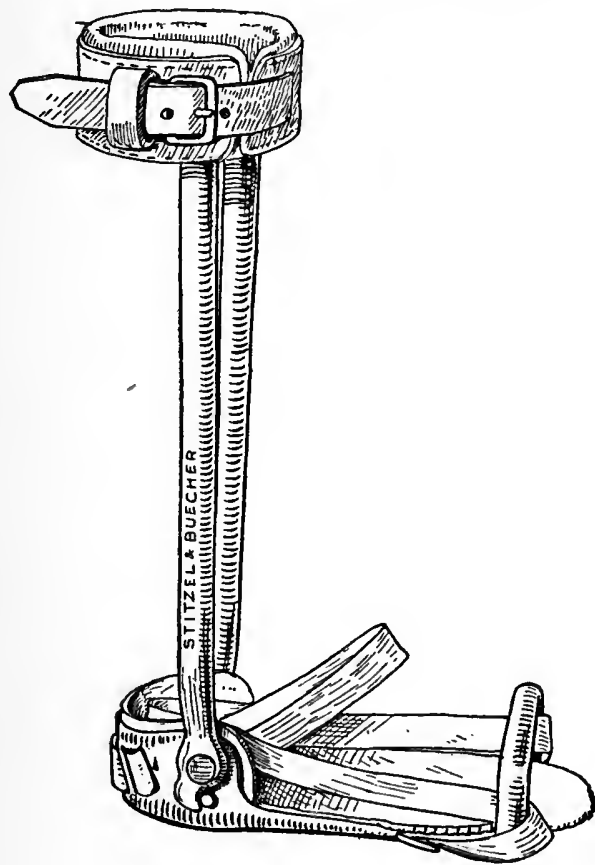

Fig. 8I. Night-Shoe

An apparatus for retaining the foot in the right-angled position while the patient is in bed. The heel-strap, secured to buckles on the bottom of the foot-plate, passes over the bar at the toe and down the sides of the foot and around the heel. The astragalar strap passes over the instep to hold the heel against the foot-plate.

ternal malleolus, and then passing it through a tumnel downward and forward, across the inner surface of the tibia and above the internal malleolus, to the scaphoid.

Where the condition presents a paralysis of the gas- 
trocnemius and the soleus, muscles from each side of the tendo Achillis may be taken and secured either to the tendon itself or directly into the os calcis. Both peroneals and the tibialis posticus and the flexor longus digitorum are all accessible. My personal experience with this operation has not been successful. It is easy to obtain good active motion in plantar flexion at the ankle joint; but as soon as this motion is attempted while the foot is weight-bearing, the insufficiency even of all these muscles as compared to the two muscles they try to supplant, becomes evident, and instead of becoming stronger with use, as one might expect, they become weaker. Moreover, in this operation one is using muscles which can ill be spared from their normal functions. Others have made artificial tendons from the hamstring muscles to the os calcis and have reported good results. This operation I have never tried.

Silk Ligaments. - Where a flail joint exists, silk ligaments may be inserted to prevent foot-drop. This will prevent the dragging of the toes and greatly assist in walking. The operation is a simple one. Number I6 or I8 silk is used. It is best to boil it first in bichloride for an hour and then in plain water for fifteen minutes and dip in boiling paraffin shortly before it is used. Two ligaments are placed, each having two strands of the silk. One extends from the cuboid, under the annular ligament, to the tibia at a point two inches above its articular surface for the astragalus. The other passes from the scaphoid, also beneath the annular ligament, to 
the same point on the tibia. The bone insertions may be made through drill holes. By passing under the annular ligament, the new ligaments will not be raised up prominently at the ankle when attempts are made at dorsal flexion. All knots should be in positions where they can be well buried in subcutaneous tissue.

In the insertion of silk as ligaments, Ryerson was, I believe, the first to place them in the tendon-sheaths of the paralyzed muscles which they are to supplant. This is undoubtedly a great advance in the technique. Allison has added some ingenious details : in foot-drop he uses one continuous strand, passing it through a drilled channel in the tarsus and running one end up the sheath of the peroneus tertius and the other up the sheath of the tibialis anticus, securing both ends high up on the tibia to its periosteum; to prevent calcaneous deformity in paralysis of the gastrocnemius and soleus, he drills two holes from the plantar surface of the calcaneum upward to either side of the tendo Achillis and threads the silk through them, bringing the loop taut around the heel and securing the ends to the tibia after passing them up through the sheath of the tendon.

Astragalectomy.- Whitman's astragalectomy, which he devised for the treatment of calcaneus, is excellent also in cases of paralysis where all or nearly all the muscles crossing the ankle-joint are powerless. While this operation allows of some movement, it prevents the extremes of either flexion or extension and thus facilitates walking, making it as easy as does an ankle brace which 
allows of slight motion. Moreover, it firmly holds the foot in the antero-posterior plane. It also increases the development of the foot, since it is used quite freely.

Whether performed for calcaneus or for a flail anklejoint, the operation is practically the same. The steps of the operation are as follows: "An Esmarc bandage having been applied, an incision is made from a point about one inch above the external malleolus midway between it and the tendo Achillis, passing downward to the attachment of the tendo Achillis, forward below the extremity of the malleolus and over the dorsum of the foot to the external surface of the head of the astragalus. The sheaths of the peroneal tendons which are exposed at once are opened, the tendons divided below the malleolus and drawn backward. One next divides the bands of the external lateral ligament, and the foot being somewhat adducted, the interosseous ligament is divided. On further inversion, the tissues being retracted, one may with scissors free the head of the astragalus from its attachments to the navicular, and forcibly twisting it outward, break off the cartilaginous margin to, which the internal and posterior ligaments that can not be reached are attached. One then prepares the new articulation. A thin section is removed from the lateral aspects of the adjoining os calcis and cuboid bones, and from the internal surface of the external malleolus, which may be further shaped to secure accurate apposition. The same, but more difficult, procedure is undertaken on the inner side. One thoroughly separates the internal lateral liga- 
ment from the malleolus in order to permit complete backward displacement, then removes the cartilage from its inner surface. With a periosteal elevator the strong inferior calcaneo-navicular ligament is detached sufficiently to permit the malleolus to sink in behind or slightly to overlap the navicular. The two peroneal tendons, thoroughly freed from their attachments to the fibula, are then passed through the base of the tendo Achillis from within outward and are sutured to it and to the periosteum of the os calcis as well, at a sufficient tension to hold the foot in moderate plantar flexion. The tendo Achillis is sometimes overlapped and sutured as an aid in restraining deformity. The malleoli are then forced forward and accurately adjusted to the new articulation and the wound closed with catgut, reënforced with several silk sutures. The foot, carefully supported in its attitude of backward displacement and moderate plantar flexion, is thickly covered with sterilized sheet wadding and fixed by a light plaster bandage, particular care being taken to exert only the slightest constriction. The leg is then brought to a right angle with the thigh and the plaster bandage is continued over the thigh, reënforced by a band of steel in the popliteal region. The limb is suspended for several days or a week, the aim being to relax tension and to lessen the congestion. The plaster bandage fixing the limb in flexion at the knee remains for several weeks until immediate repair is complete, a section being removed over the wound to permit inspection at the end of a week. It is then replaced by 
2 I6 DISEASES AND DEFORMITIES OF THE FOOT

one that reaches only to the knee, holding the foot in moderate plantar flexion, the sole being made level by the insertion of a piece of cork. The plaster support is worn for about six months, the longer the better, since the patient must bear weight on the front of the foot. Success in this treatment is directly dependent upon the accuracy with which its details are carried out. The most important of these is secure fixation in complete backward displacement." 
TUBERCULOUS AND GONORRHEAL DISEASE

TUBERCULOUS DISEASE OF THE FOOT

THE primary focus may be in the tibia, fibula, one of the tarsal bones, or in the synovial membrane. Clinically it is usually found to be limited to one bone or to involve one or more joints together with the synovial membrane. The tendon sheaths may be infected, especially that of tibialis anticus, from their insertion into an infected bone. The order of frequency in which the bones were attacked in I23I cases collected by Whitman, were: calcaneum, astragalus, cuboid, scaphoid and metatarsals, cuneiform bones, malleoli, phalanges. The calcaneum and the astragalus are primarily infected much more than any of the other bones.

The etiology and pathology are the same as bone tuberculosis elsewhere.

Diagnosis. - The history will be that of a limp with more or less pain, especially at night, alterations in the contour of the foot and sometimes sinus formation. In children it is usually the limp for which consultation is sought.

Limp. - At the beginning this will be intermittent. The infected foot will unconsciously be favored in such a way as to relieve the infected areas from weight- 
bearing and from movement. Thus, if the focus is in the astragalus or in the os calcis, the foot will be used in the position of slight equinus; if the medio-tarsal joint is the location of the infection, the deformity will be that of abduction, resembling that of valgus; and if the focus is in the forefoot, the child will walk on the heel.

Pain will not be a prominent symptom during the early stages, but later, especially in adults, it may be severe. In children the pain may be enough to disturb sleep and to cause the patient to desist from romping as much as usual. There is little of value as a help in diagnosis, in the designated site of the pain. It may be along the inner or outer side of the foot. Sometimes pressure over the site of the infection will produce pain, but the absence of such a painful spot is of little or no value. The character of the pain is not distinctive of this disease. In adults, when the medio-tarsal joint is involved, the pain may resemble very closely that accompanying flatfoot; a strained, sore, aching feeling rather than a sharp, severe, acute pain.

Contour. - In the earliest stages even, a careful observer, on comparing the two feet, will note differences in them. Depressions in the normal foot will be filled in, partially or completely, in the other. If the synovial membrane is infected early, there will be a puffy appearance wherever the infected membrane is not confined securely by the over-lying structures; as on each side of the tendo Achillis, below and in front of the malleoli, over the calcaneo-cuboid joint, or the astragalo-scaphoid 
joint, or in front of the ankle on either side of the extensor tendons. The atrophy of the calf-muscles is an early sign, but in the early stages is not recognizable except by the use of the tape-measure.

The consistence of the swelling will differentiate between bony enlargement, induration of the soft parts, synovitis and abscess formation. That is to say an attempt should always be made to make such a differentiation, but experience alone can teach the comparative points in density, in fluctuation and in "feel."

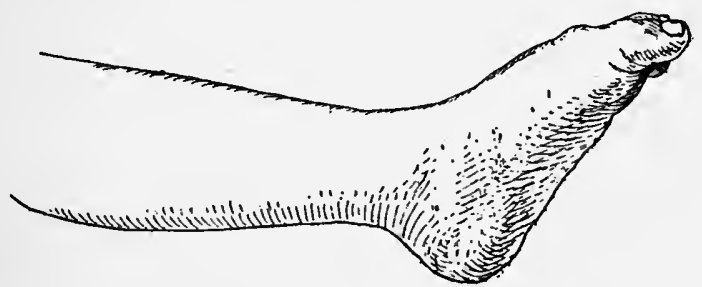

Fig. 82. TUberculous Foot

This drawing, from a photograph, shows the fusiform enlargement, pointing of the toes and the equinus position.

Deformity.-If the ankle joint is infected, there will gradually be assumed a position of equinus; if the calcaneo-astragaloid joint, a position of eversion; and if the medio-tarsal point, especially the astragalo-scaphoid joint, a position of abduction. With the increase of the amount of tissue involved, the entire ankle and foot become involved, fusiform in shape, with toes pointed downward and slightly outward; and the possible appearance of sinuses, in almost any location, will complete the picture. 
When one malleolus is attacked, it may be so enlarged as to give the foot the appearance of having been badly treated after a fracture. The foot seems to be dislocated at the ankle in the opposite direction. As long as the ankle joint is free of infection there will be no muscular spasm associated with its movements. Frequently pressure over the malleolus will give pain.

Heat.- - Heat will be present during the activity of the disease. It is best determined by first laying the hand on the sound foot until its temperature is determined, as compared with the temperature of the hand; and then placing the hand on the suspected foot and noting any difference in temperature. If one becomes adept in this examination for slight differences in the temperature of the two feet, he will have an excellent aid to the determination of the efficiency of any treatment being pursued. Redness is not present except at some point in advance of the opening of an abscess. The superficial veins may be much more marked than in the other foot.

Muscular Spasm. - This will invariably be present whenever a joint becomes involved. Any movement, active or passive, of such a joint will elicit this spasm. Whether the spasm occurs at the beginning of movement, or not until the normal limitations of movement at that joint have been reached, will depend upon the exact location of the infected area and its extent. If this area is extensive, there will be no movement in the joint, it will be absolutely immobilized by the muscular spasm; if this area is very limited, movements may be 
free until the limitations are approached, when they will be found to be halted before they are in the normal foot; the range of motion is shortened. This spasm is the same involuntary protection of a diseased condition, or an injury, which is found in so many instances. The diagnostic value of this spasm was, I believe, not fully recognized until studied by the orthopedic surgeon. To-day the general surgeon, in a case of appendicitis for instance, is often guided more by the spasm than by any other one sign or symptom.

In examining a joint for muscular spasm, it is necessary that the examiner be ready and able to recognize the slightest restriction to movement, its sharp, sudden appearance, its muscular rather than bony "feel," and the invariableness with which this spasm always appears when that particular angle of movement is reached. There is no better indication of the progress, or the lack of progress, being made in the treatment of tuberculous arthritis, than the increase or decrease of the muscular spasm. In severe cases there will be no movement discernible, the joint will seem to be ankylosed; but a patient, careful examination will disclose the presence of a spasm while attempts at movement are being made; and spasm once felt rules out bony ankylosis, regardless of whether every other test, even X-rays, seem to point toward bony ankylosis. When a muscle passes over two or more joints, and one of these joints is the site of a tuberculous infection, it will limit only the movement of the diseased joint and will not affect movement 
in the other joints, unless the movements in the healthy joints tend to convey some movement to the diseased joint. Thus if the medio-tarsal joint is the subject. of infection, the ankle joint may be freely moved if none of the movements is extended to the medio-tarsal. It is the failure to recognize this fact which sometimes leads to an erroneous diagnosis. After examining the sound foot, so as to become familiar with the range of movements it enjoys, each of the three axes of motions in the suspected foot should be examined separately. Early in the disease there is not likely to be absolute immobilization from the spasm, but only a limitation of movement. Later this muscular spasm may cause complete fixation.

Mensuration.- This will disclose the atrophy of the leg, more marked in disease of the ankle joint and of the subastragaloid than of the medio-tarsal, and also the swelling of the foot. All measurements must be made at the same distance from corresponding points on each leg and foot. This atrophy is present early in the disease, increases as the disease progresses, and is always greater than would accompany disuse alone.

$X$-rays. - The radiograph of a suspected foot will be of inestimable value and should always be had, if possible, before committing oneself to a positive diagnosis. In very early cases, however, if all the clinical signs and the history point to a tuberculous infection, even though the radiograph is negative, I should advise that the treatment be directed toward this disease. When the symp- 
toms are pronounced, the absence of confirmation by the radiograph is, of course, of the greatest importance.

Tuberculin Test.- The various tests with tuberculin may be used with the same advantage obtained in its use for diagnostic purposes in other suspected regions. A positive reaction may come from the presence of a focus in some other part of the body.

Differential Diagnosis. - The following table for differential diagnosis is open to criticism in many respects, but it would manifestly be impossible to tabulate the many exceptions which may be taken to the statements as we have set them forth. Probably few physicians of wide experience would agree in all respects with this or any other similar table.

\begin{tabular}{|c|c|c|c|c|c|c|}
\hline & FLAT-FOOT & $\begin{array}{c}\text { TUBERCU- } \\
\text { LOUS } \\
\text { ARTHRITIS }\end{array}$ & $\begin{array}{c}\text { GONOR- } \\
\text { RHEAL } \\
\text { ARTHRITIS }\end{array}$ & \begin{tabular}{|c|} 
RHRUMA- \\
TOID \\
ARTHRITIS
\end{tabular} & GouT & $\begin{array}{l}\text { PYOGENIC } \\
\text { INPBCTION }\end{array}$ \\
\hline & Bilateral & Unilateral & Unilateral & Unilateral & Unilatera & Unilateral \\
\hline Age & $10-30$ & $2-20$ & $15-25$ & $2-40$ & $35-60$ & Any age \\
\hline Sex & Male & Either & Male & Female & Male & Either \\
\hline $\begin{array}{l}\text { Previous } \\
\text { attacks }\end{array}$ & None & None & Likely & Likely & Likely & None \\
\hline Onset & Slow & Slow & $\begin{array}{l}\text { Slow or } \\
\text { rapid }\end{array}$ & Slow & Rapid & Rapid \\
\hline $\begin{array}{l}\text { Constitu- } \\
\text { tional } \\
\text { symptoms }\end{array}$ & Absent & Absent & Slight & Absent & Slight & Marked \\
\hline Location & $\begin{array}{l}\text { Astragalo- } \\
\text { scaphoid } \\
\text { joint }\end{array}$ & $\begin{array}{l}\text { Infected } \\
\text { area }\end{array}$ & $\begin{array}{l}\text { Tendon } \\
\text { sheaths } \\
\text { Bursae } \\
\text { Plantar } \\
\text { fascia }\end{array}$ & $\begin{array}{l}\text { Involved } \\
\text { joints }\end{array}$ & Great toe & $\begin{array}{l}\text { Infected } \\
\text { joints }\end{array}$ \\
\hline
\end{tabular}




\begin{tabular}{|c|c|c|c|c|c|c|}
\hline & FLAT-FœT & $\begin{array}{c}\text { TUBERCU- } \\
\text { LOUS } \\
\text { ART H RITIS }\end{array}$ & $\begin{array}{c}\text { GONOR- } \\
\text { RHBAL } \\
\text { ARTHRITIS }\end{array}$ & \begin{tabular}{|c|} 
RHEUMA- \\
TOID \\
ARTHRITIS
\end{tabular} & Gour & $\begin{array}{l}\text { PYoggnic } \\
\text { INFECTION }\end{array}$ \\
\hline $\begin{array}{l}\text { Position } \\
\text { of foot }\end{array}$ & $\begin{array}{l}\text { Everted } \\
\text { abducted }\end{array}$ & $\begin{array}{l}\text { Everted } \\
\text { abducted } \\
\text { plantar- } \\
\text { flexed }\end{array}$ & $\begin{array}{l}\text { Everted } \\
\text { abducted }\end{array}$ & $\begin{array}{l}\text { About } \\
\text { normal }\end{array}$ & $\begin{array}{l}\text { Extension } \\
\text { great toe }\end{array}$ & $\begin{array}{l}\text { Varies } \\
\text { widely }\end{array}$ \\
\hline Swelling & $\begin{array}{l}\text { Inner } \\
\text { border of } \\
\text { foot }\end{array}$ & Fusiform & Diffuse & $\begin{array}{l}\text { May be } \\
\text { absent }\end{array}$ & Great toe & Varies \\
\hline Color & Purplish & White & Normal & Normal & Purplish & Red \\
\hline Heat & Absent & Slight & Absent & Absent & Slight & Marked \\
\hline $\begin{array}{l}\text { Tender- } \\
\text { ness }\end{array}$ & $\begin{array}{l}\text { Over } \\
\text { Hunter's } \\
\text { capsule }\end{array}$ & $\begin{array}{l}\text { Slight, } \\
\text { over in- } \\
\text { fected } \\
\text { area }\end{array}$ & $\begin{array}{l}\text { Over } \\
\text { bursae } \\
\text { tendon } \\
\text { sheaths } \\
\text { plantar } \\
\text { fascia } \\
\end{array}$ & $\begin{array}{l}\text { Not } \\
\text { marked }\end{array}$ & $\begin{array}{l}\text { Over me- } \\
\text { tacarpal- } \\
\text { phalan- } \\
\text { geal joint } \\
\text { of large } \\
\text { toe } \\
\end{array}$ & $\begin{array}{l}\text { Inflamed } \\
\text { area }\end{array}$ \\
\hline Pain & $\begin{array}{l}\text { Only after } \\
\text { use } \\
\text { Soreness } \\
\text { Bruise }\end{array}$ & $\begin{array}{l}\text { Slight but } \\
\text { continu- } \\
\text { ous } \\
\text { Worse at } \\
\text { night } \\
\text { Sharp } \\
\end{array}$ & $\begin{array}{l}\text { After use } \\
\text { Ache }\end{array}$ & $\begin{array}{l}\text { Continu- } \\
\text { ous } \\
\text { Increased } \\
\text { by use } \\
\text { Dull }\end{array}$ & $\begin{array}{l}\text { Worse at } \\
\text { night } \\
\text { Constrict- } \\
\text { ing char- } \\
\text { acter }\end{array}$ & $\begin{array}{l}\text { Continu- } \\
\text { ous } \\
\text { throbbing }\end{array}$ \\
\hline $\begin{array}{l}\text { Muscular } \\
\text { spasm }\end{array}$ & $\begin{array}{l}\text { Peroneus } \\
\text { longus } \\
\text { and brevis }\end{array}$ & $\begin{array}{l}\text { Most } \\
\text { marked: } \\
\text { P. longus } \\
\text { and brevis } \\
\text { or gastroc- } \\
\text { nemius }\end{array}$ & $\begin{array}{l}\text { Peroneal } \\
\text { muscles }\end{array}$ & $\begin{array}{l}\text { May be } \\
\text { absent }\end{array}$ & $\begin{array}{l}\text { Extensor } \\
\text { of great } \\
\text { toe }\end{array}$ & $\begin{array}{l}\text { Marked in } \\
\text { any } \\
\text { movement } \\
\text { of involve } \\
\text { joints }\end{array}$ \\
\hline Blood test & Negative & $\begin{array}{l}\text { Tuber- } \\
\text { culin } \\
\text { reaction }\end{array}$ & $\begin{array}{l}\text { Compli- } \\
\text { ment fixa- } \\
\text { tion for } \\
\text { gonococci }\end{array}$ & $\begin{array}{l}\text { Compli- } \\
\text { ment fixa- } \\
\text { tion for } \\
\text { auto-infec- } \\
\text { tious mi- } \\
\text { cro-organ- } \\
\text { ism from } \\
\text { teeth or } \\
\text { elsewhere }\end{array}$ & Negative & Negative \\
\hline $\begin{array}{l}\text { Condi- } \\
\text { tions to } \\
\text { be sought } \\
\text { elsewhere }\end{array}$ & None & $\begin{array}{l}\text { Atrophy } \\
\text { of leg } \\
\text { muscles }\end{array}$ & $\begin{array}{l}\text { Gonor- } \\
\text { rheal uri- } \\
\text { thritis or } \\
\text { ophthal- } \\
\text { mia }\end{array}$ & $\begin{array}{l}\text { Results of } \\
\text { attacks in } \\
\text { other } \\
\text { joints }\end{array}$ & Tophi & None \\
\hline
\end{tabular}




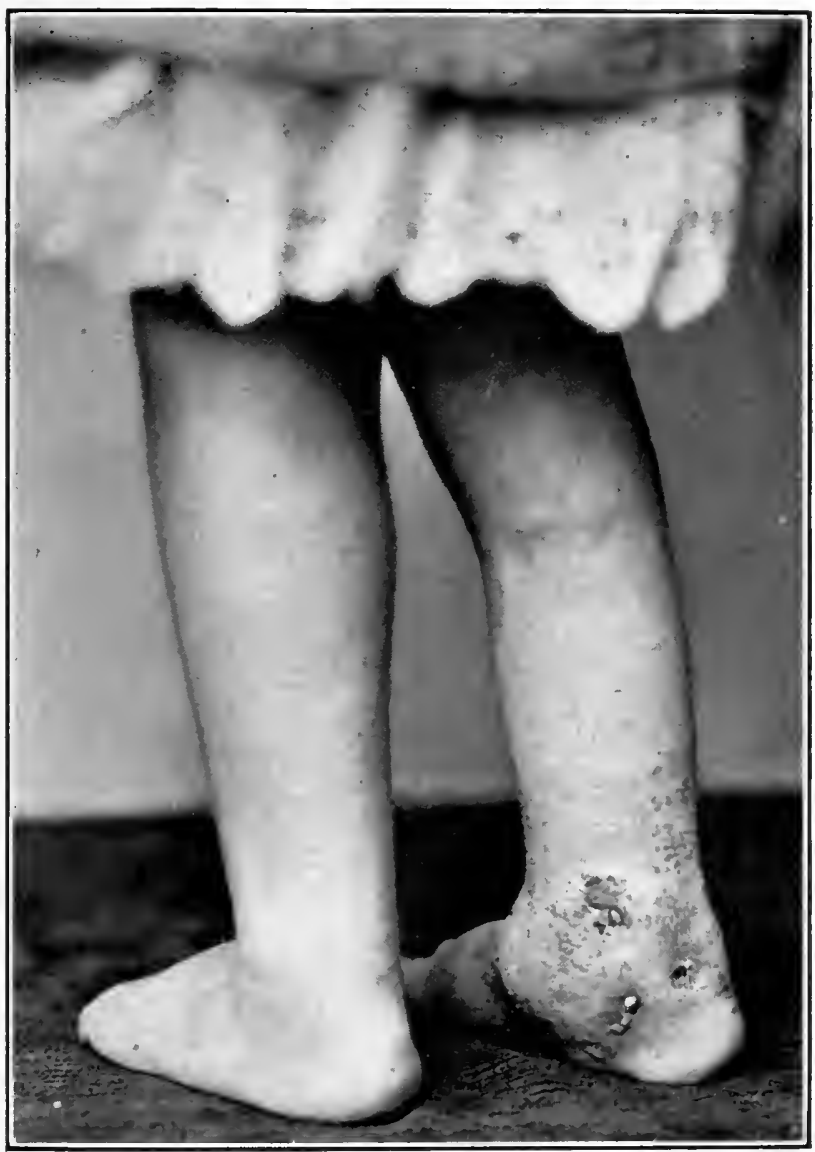

Plate IV.-Tlberculoes Disbase of the Foot

The disease has extended so as to involve the tibia, astragalus, calcaneum and scaphoid. At the time thls photograph was taken the equlnus was practically overcome but valgus and eversion are still marked. The sinuses are discharging freely and spasm is evident upon the least attempt at passlve motiots. An ankle-brace, crutches, and helio-therapy, witl extraurban hospital care, arrested the disease, healed the sinuses and reduced all deformities. 



\section{TREATMENT}

Immobilization. - It is impossible to obtain traction in order to prevent inter-articular pressure; so that immobilization, with the removal of weight-bearing during the acute stage, is the object to be sought. Immobilization may be obtained by using plaster-of-Paris, a steel brace, molded leather, porous plastic felt, or silicate bandages. In the hands of those who are not expert orthopedic surgeons, the plaster-of-Paris is probably the most satisfactory to use, especially while the disease is active and while deformity and swelling exist. If the ankle joint or the subastragaloid is involved, the knee should be included in the bandage so as to insure control over the gastrocnemius. Care must be taken to put the foot up in the best position obtainable without using too much force. If some equinus and abduction are present, they need not be corrected at once, as these deformities will become less as the foot improves, and any violence to the diseased area is to be avoided. If sinuses exist, fenestra must be cut in the plaster to permit of their being dressed. The plaster should be changed about every two weeks if deformity and swelling are present, so as to allow further correction of the position and the better fitting of the bandage as the swelling subsides. When deformity and swelling are absent, the bandage needs to be changed but once a month. While the disease is active, the foot should not be allowed to hang down. Confinement in bed is not necessary nor 
advisable, but the foot should be kept elevated the greater part of the time.

With the reduction of deformity, disappearance of swelling and lessening of spasm, and when these signs are not greatly marked at the first consultation, ambulatory treatment may be tried. It is at this stage that a brace is especially indicated: if properly fitted, it is not only more comfortable than plaster but better admits a close observation of the progress being made. The slightest increase of spasm is indication of insufficient protection. It should not be necessary to wait for the grosser signs, actual deformity or immobilization or swelling, to recognize Nature's assertion that we are not properly caring for the condition. At the first hint, an increase of spasm, a shorter range of motion, the treatment must be changed.

Braces. - There are three braces which may be used in tuberculous disease of the foot: the ankle brace, the long leg brace of Shaffer's, and the Thomas knee brace.

The ankle brace consists of a foot piece with two side bars extending to the knee and has no joints. Instead of a calf band it has a band connecting the tops of the leg-bars and passing in front of the tibia, with a strap passing around the calf and completing the circumference of the leg. The foot-piece must be made to fit accurately the bottom of the foot so that it will act as a perfect, immobilizing splint. It may be made over a plaster cast of the foot, or made from an outline of the foot and fitted, altered, and refitted until perfectly satis- 
factory. The use of a cast is recommended for the inexperienced surgeon, especially if the brace is to be made by a mechanic who is not an expert in this work. The side bars are formed to correspond to the outlines of the leg and are then welded to the foot-piece. They must not be riveted to the anterior cross-bar until they have been fitted to the leg after the welding, as it is impossible to alter them after completion of the brace without freeing them from this cross-bar, because any change in their outline must be felt in other parts of the apparatus. The cross-bar should lie across the lower part of the tibial tubercle, should be well padded, and should fit snugly. The object of having this steel bar in front is to absolutely prevent any dorsal flexion, which is not possible with only a strap in this location. A strap to buckle over the ankle is riveted to the bottom of the foot-piece, just behind the line of the leg-bars. This strap, together with a laced shoe going on over the brace, will securely hold the foot against the foot-piece, and render immobilization complete. During the stage of convalescence, if the disease has been confined to the forefoot, an ankle brace may be applied permitting movement at the ankle.

Campbell Brace.-The long leg brace which may be used for these cases is called the Campbell brace by Dr. Shaffer. The name is derived from that of the first patient for whom he devised it, just as he named the Condon brace after the first patient to wear one. The principle of this brace is to transfer the concussion of 


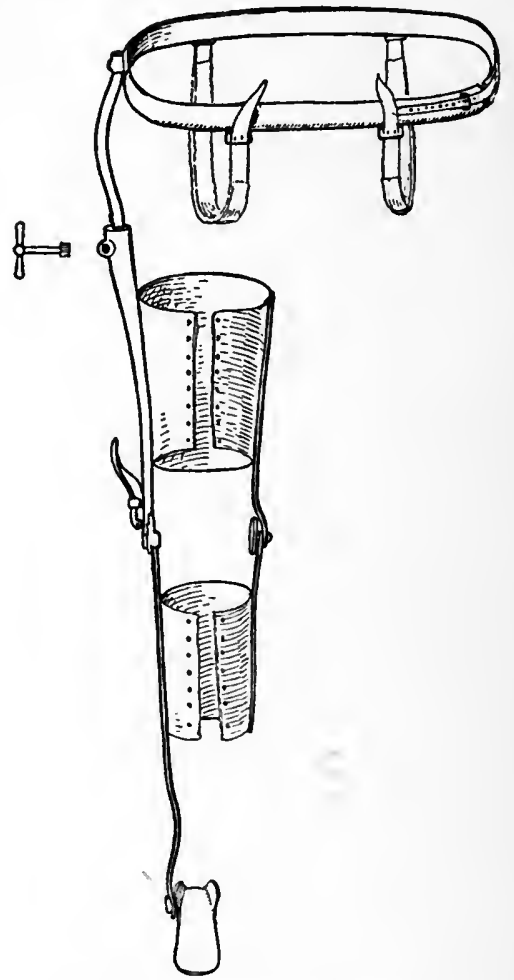

Fig. 83. Campbell Brace

The pelvic band and the perineal straps are properly adjusted, the sole plate brought against the bottom of the foot and the shoe put on and laced up. Extension by means of the ratchet in the thigh-bar is then applied. This pushes the shoe away from, below, the foot. The thigh and calf bands are then laced. All weightbearing is removed from the foot. The weight is transferred from the body through the tuberosities of the ischii, the perineal bands, the pelvic band, the long outside bar of the brace, to the footpiece. The lock at the knee permits bending that joint when sitting; desirable not only for convenience, but as an important factor in preserving the health of the knee and the leg and thus a factor in curing the foot. 
each step and the weight of the body, if desirable, from the foot to the tuberosity of the ischium. It consists of a long outside leg-bar, reaching from a foot-plate to a pelvic band and having a joint at the knee. A short inside bar reaches from the middle of the leg up to the inner side of the thigh at a point two and a half inches below the perineum, and is connected with the outside bar by a posterior calf and a thigh bar. In the outside bar, about opposite the middle of the thigh, is a ratchet, which permits of separation of the pelvic band and the foot-piece. The apparatus is applied with the patient lying on a couch: the pelvic band is adjusted, strapped, and the peroneals buckled into place; then by means of the ratchet, the foot-piece is brought up against the sole of the foot and the shoe is put on and laced up; with the leg straight, the ratchet is made to push the soleplate, and with it the shoe, away from the sole of the foot; then the straps for the thigh and leg are buckled, and the patient allowed to get up. When properly adjusted, there can be very little weight conveyed through the foot and none of the concussion of walking will be felt at the diseased area.

The Thomas Knee Brace also transfers the weightbearing and the concussion of walking to the tuberosity of the ischium. It consists of an iron ring, made from an outline obtained with a lead tape, so as to fit snugly around the thigh up against the ischeal tuberosity and at about the top of the great trochanter on the outer side. Provision must be made for covering the ring 


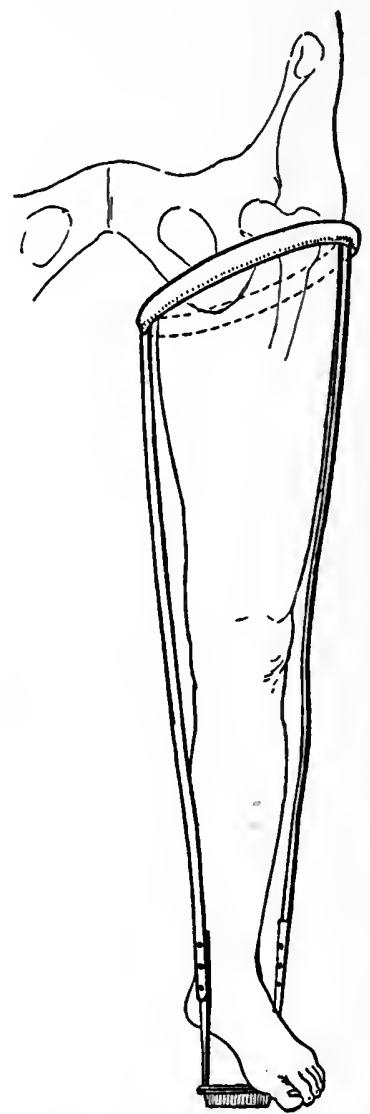

Fig. 84. Thomas Knee-Brace

This simple and economical ambulatory brace is suitable for use in disease of the foot. It removes weight-bearing by transmitting the weight from the pelvis, through the tuberosity of the ischium, to the padded iron ring encircling the thigh. From this ring the weight is taken by the upright bars, to the cross-piece below the foot. The length of the uprights may be adjusted by the screws in their lower part. Straps are placed about the thigh and leg, and a broad band supports the back of the knee. A high shoe is worn on the sound foot. 
with leather and for padding it where it comes against the tuberosity. Extending down from the ring on each side is a steel rod having a crossbar connecting the lower ends. This crossbar should be about an inch and a half below the sole of the foot. The illustration shows how a leather "shoe" may be attached to the crossbar.

Whether a brace, and what kind of brace, or whether plaster-of-Paris is to be used, must depend upon the exact site of the diseased process; its extent; the presence of deformity; the condition of the patient, his age, weight, and general activity; and the control over the case which the surgeon can be sure of maintaining. With the Thomas brace a high shoe should be worn on the sound foot and sometimes crutches should be provided. With Shaffer's brace crutches are frequently needed. With the ankle brace it is frequently necessary to interdict all walking and standing until convalescence is established. All of these matters must depend on the ability of the surgeon to recognize the slightest changes in the condition and on his promptness in making changes in treatment to meet the indications.

Bier's Treatment.-Congestion, theoretically carrying to the diseased area an increased supply of blood with its bactericidal properties, should be of great value in the treatment of these cases. While some of the reports of cases treated by this method seem to be most encouraging, many surgeons have been disappointed in its use. Perhaps one great source of failure in using congestion, is to be found in the too great dependence 
which some have placed on it. Its use is sometimes accompanied by a relaxation of the minute care which should always be given to the general and to the local treatment. If the bactericidal power of the blood is low, or is allowed to deteriorate, the increased supply of such blood may not be equal to the normal supply of blood which has been carefully built up by general treatment. So fixation and relief. from functionating may be more powerful in preventing an increase in the local infection than congestion, and I do not believe they can be slighted without disastrous results. Bier's treatment should be regarded as an adjunct to other treatment.

The object of this treatment is to obtain hyperemia; redness, heat, and swelling. Stasis is to be avoided as injurious. The bandage should be applied about the middle third of the thigh. It should not cause any discomfort. If a rubber bandage is used it should be applied over a cotton or flannel bandage, so as to avoid any effect of the rubber on the skin. If an elastic bandage, not of pure rubber, is used, it should not have a selvage; it must be equally elastic throughout. Before applying the bandage, note the condition of the skin; its temperature and color, the superficial veins, the appearance of any sinuses, and the pulse at the ankle.

There are two distinct methods of proceeding. A degree of congestion may be obtained in which the veins are made more prominent, fuller, but they remain easily compressible by the finger, and the skin becomes just barely blue-red, not cyanotic. The bandage is worn for 
five or six hours every day, the time being reduced to three hours after a week or ten days and after that to but one hour a day. In the other method, called the "intensification congestion method," the bandage is applied more firmly and the skin becomes bluish-red. There is some swelling but no edema. A prickly sensation may be felt, but no pain. Cantile thinks this is the better method of treatment in experienced hands.

Tuberculin Treatment.- The therapeutic value of injections of tuberculin, in the treatment of bone tuberculosis, has been very widely tested during the past six years. The consensus of opinion, among American orthopedic surgeons at least, seems to be against its having any pronounced value. I have given it a careful trial at the New York State Hospital for Crippled and Deformed Children and also in private practice, having used it in over forty cases, in most of which the opsonic index was regularly ascertained, the clinical signs recorded once a week or at least once a fortnight, and notes kept of the general condition, including weight, appetite, urinalysis, and general appearance. A report of over twenty of these cases was published in the Journal of The American Orthopedic Association in 1909, in conjunction with Hastings, who undertook the laboratory work. The opinion which I now hold regarding tuberculin in these cases is, that in most instances it is a valuable tonic; that it is not injurious in any way if constant watch is kept for the slightest reaction and anything more marked than a slight malaise avoided; that 
its influence on the local lesion is dependent on any improvement in the general condition, appetite, etc.; and that it in no wise warrants any less careful attention to details of other treatment. In some cases it seemed to shorten the time of treatment, but not so markedly that it could be highly recommended. It may be that this line of treatment will yet be perfected so that it may be used to greater advantage.

It may be administered during any stage of the disease. The initial dose should be from .ooor to $.0002 \mathrm{mg}$. or $\mathrm{m} 3$ of a solution, I c.c. of which equals .0000or gm. The next dose may be $m 5$, and so on up until .oor mg. or $\mathrm{m} \mathrm{I} \mathrm{I} / 2$ of a solution, I c.c. of which equals .oooor $\mathrm{gm}$. is reached.

Until the dose of .oor $\mathrm{mg}$. is given, the inoculations should be given every three or four days, twice a week. After that every five days, or once a week. When the dose has reached .or mg. or $m 2$ of a solution, I c.c. of which equals .ooor gm., the vaccinations should be made once in ten days and the greatest of care should be taken to guard against reactions. Larger doses are not advisable. The treatment should be interrupted for a month or two and the second series of inoculations should be begun with from .0001 to $.0002 \mathrm{mg}$.

A slight local reaction, whether simply an erythematous condition of the skin or an indurated mass at the site of injections is of no consequence, but injection should be made elsewhere until the part is again normal.

The temperature should be taken every four hours, 
for forty-eight hours before and after each inoculation. A sudden or unusual rise in temperature had better be followed by a much reduced dosage or an omitted dosage.

Constitutional symptoms, such as nausea, chilly sensations and headache, occurring within forty-eight hours of a vaccination demand a cutting of the dosage by onehalf, and a slower rate of progression.

The opsonic index is not to be used as a guide to the removal of mechanical apparatus.

Tuberculous individuals show variations in the indices which are more marked than in the normal individual. Inoculations are to be continued regardless of the indices; the clinical symptoms alone to be the guide. Tuberculin can be safely given without ascertaining a single opsonic index.

Heliotherapy.-The sun's rays may be used with decided advantage if the treatment is carried out in a conscientious manner. The indifferent exposure of a tuberculous foot to the sunlight cannot be of value. Probably the therapeutic value derived from the sun comes from a stimulation of metabolism and the diseased area is benefited more through a constitutional effect than through any direct local effect. Rollier's method is to begin with a short exposure of one part and then to increase at each treatment the time of exposure of that part and add a new area for a short original exposure. Thus at the third treatment the left leg may be exposed thirty minutes, the left thigh twenty minutes and the 
right leg ten minutes. This is continued until the entire body is exposed for one or two hours a day, or sometimes longer. It would not be possible to follow out any set of rules in all cases nor in all localities. The object to be sought is a tanning of the skin. Rollier believes that he can prognose with some certainty the value the treatment will have in any individual case by the appearance of this tanning. Not only will some skins $\tan$ easier than others and be less likely to be burned, but the condition of the atmosphere, effecting the penetration of the rays, the altitude of the sun, effecting the thickness of the layer of the atmosphere through which the rays must travel, and the surrounding conditions which effect the reflection of the rays must be factors of importance in determining the manner of prescribing. The head should be protected by a shade-hat or a parasol. In dry climates the entire body may be exposed in low temperatures. The protection of the foot by a perfectly fitting brace must not be interrupted during this treatment.

Sea-bathing.- If one is treating a tuberculous foot at the sea shore, sea-bathing is generally to be recommended. Not only is it a wholesome stimulation to metabolism but I believe the exposure of a sinus to the sea water is in itself beneficial. The foot should be well protected by a brace which may be dried and reapplied after the bath or a brace may be used especially for bathing. Sinuses may be protected by one or two layers of gauze. 
Constitutional Treatment.- In tuberculous disease of the bones of the foot, as in that disease of any of the bones and joints, the general condition of the patient is as essential as it is in tuberculosis of the lungs. The best of air and of food is essential for the preparation of the opsonins and other body-forces for the conquering of the disease. The best air is always out-of-door air, preferably country air, and each inspiration is of benefit. Therefore an out-of-door life, night as well as day, is desirable. This is the treatment followed at the New York State Hospital for Crippled and Deformed Children for all of these cases. Regardless of weather conditions the children are out of doors night and day, protection, of course, being provided against severe winds, rain, and snow. Naturally, sufficient and proper clothing must be used to preserve the temperature of all parts of the body. Since this night and day fresh air treatment was adopted four years ago, there has been an undoubted improvement in the treatment of these cases of bone tuberculosis.

Opinion seems to be divided as to the advantages of sea air over mountain air, some claiming superiority of one and some of the other. I do not believe there has been a sufficient amount of data collected, or sufficiently careful analysis made of the air in different localities, for the drawing up of positive statements. From my personal observations I am inclined to believe that with the same surgical treatment many cases do equally well in either environment, others do well at the sea shore 
but would do better at an altitude or vice versa, and yet others progress very poorly at one place who would be benefited at the other place. In other words, for many, perhaps most, of these cases, there is a choice between sea air and mountain air, but at present there is no method of determining that choice except by trial.

Theoretically a meat diet might seem to be advisable, the carnivorous animals being less subject to tuberculous infection than the herbivorous; but experiments I have carried out have failed to prove anything positive and a mixed diet of nourishing food is all I can recommend.

Operations. - In children this is so seldom indicated that it may be stated, as a rule, Do not operate. In adults new factors enter into the question, such as the probable duration of treatment and the final result of prolonged treatment as compared with operation. The length of time consumed by conservative treatment is never under eighteen months, seldom under two years, and more frequently three or more years. The amount of incapacity for work during treatment is not only frequently of grave importance in adults; but complete idleness, of such long duration, is more or less demoralizing to most characters.

The result of conservative treatment in the adult, must be dependent to a great extent, on the ability of the patient to follow the orders of the surgeon. If it is evident that the patient will not be able to follow the necessary instructions, including both local and general treatment, in their minutest details, then I believe it to 
be wrong to enter upon them. The patient is too often allowed to "try" conservative treatment, when if he was given distinctly to understand that it would probably be years before he could discard all protection to the foot; and that during these years it would be in a greater or less vulnerable condition, likely to become worse through some minor accident; and that most of the time he would have to pay special attention to his general health, he would probably elect to have some operative interference, even an amputation, were it offered him. In the early stages, when the pain may not be excessive, it is easy to persuade the patient that plaster-of-Paris will probably cure the condition, but must be worn for a "long time." Several months is a "long time"; but if he must submit, he will. Tell him it will take three or four years, with the plaster or a brace, and offer him the alternative of an operation; and, I believe, most busy men, who have not much money and have some one or more persons dependent upon them, would not delay long in selecting the radical treatment. The mistake lies in undertaking to give a patient a "try" at conservative treatment, when we know that he can not continue it in all its details for more than a year. When the patient has an abundance of means, will not be made "sick with worry," and is one to whom time is little object, the conditions are entirely different.

With a single focus, and that limited to one bone, excision is indicated. Anything less, such as opening and curetting is inadvisable. If the disease is more 
extensive, then every bone which is surely infected should be excised. And with this excision, all possible infected soft tissue should be cut away. The incisions should be made through healthy tissue. It is of less importance to follow the procedure of any classical operation for the excision of this or that bone or part of the foot, than it is to accomplish that for which the operation is performed, the eradication of all diseased tissue. The more thorough the operation, the less the danger of complications, such as meningitis, and the shorter will be the convalescence. During the early stage, operation need produce but little mutilization of the foot, while during the latter stages amputation may be necessary.

Sinuses.-Abscesses and sinuses are to be treated according to the symptoms they excite and the line of treatment being followed. Where conservative treatment is being followed, the appearance of an abscess, some time after the patient has been under observation, usually points to some fault in treatment. The foot is not receiving sufficient protection or the general health is not improving. Under efficient treatment most of these abscesses will become absorbed. It is seldom that they need to be opened or aspirated on account of their being the source of pain. Sinuses should be kept scrupulously clean so as to prevent a mixed infection. It is the mixed infection of sinuses from bone tuberculosis which is the greatest cause of amaloid changes in the internal organs. When the sinus becomes nearly dry bismuth paste may be tried to hasten its closing. I give 
Beck's formula for its composition, but would not recommend it to be used unless a very small quantity will fill the sinus and that should be injected without much pressure.

At the beginning, until all discharge of pus has ceased, the formula used is:

Bismuth subnitrate (arsenic free) ....30.0 gms. Vaseline ...............6. 60.0

Mix while boiling.

Afterward, the formula used is:

Bismuth subnitrate $\ldots \ldots \ldots \ldots \ldots \ldots 30.0$ gms.

White $\operatorname{wax} \ldots \ldots \ldots \ldots \ldots \ldots \ldots \ldots$

Soft paraffin $\ldots \ldots \ldots \ldots \ldots \ldots \ldots \ldots . \ldots \ldots$

Vaseline ................60.0

Mix while boiling.

"Care must be taken that no water should accidentally be spilled into the paste during the process of boiling, and the glass syringes must likewise be sterilized by the dry process and the plunger dipped in sterile vaseline instead of water, before charging the syringe." The fistula tract must be dried out, if possible, by packing with dry gauze before the injection.

If a case to be operated has sinuses they should be cut out, the cutting being done through healthy tissue, so as to include their entire wall. Abscesses must be emptied and their walls cut entirely out with scissors, 
and then the surfaces left free swabbed with pure carbolic followed by swabbing with alcohol.

Injections.- Injections of iodoform and glycerine, ten per cent., are frequently used in treating tuberculous disease of joints, especially after abscess formation. Instead of glycerine; ether, olive oil, and other vehicles are also used. Glycerine may be rendered sterile by adding one per cent. of carbolic acid; and the iodoform, by being steeped in a carbolic solution, one in twenty. The skin may be prepared with tincture of iodine and then frozen with ethel chloride. The needle is introduced in such a way as to avoid important structures or areas which appear to be breaking down. Any pus is first aspirated. The syringe, holding two or three ounces is then screwed on the trochar, and the injection made slowly and with considerable pressure. Upon withdrawal of the trochar, a pledget of cotton dipped in a fifty per cent. solution of alcohol is pressed against the puncture for several minutes. Then a pad is applied and firmly bound on with a bandage. Considerable pain may result, lasting in some instances for several days; but this will vary greatly in individual cases. If it seems to be advisable, the injection may be repeated in about four weeks. A combination of iodoform and glycerine is also used in the treatment of sinuses. It should be injected through an olive pointed tube, and the injections repeated at about every dressing.

Kirmisson prefers a one in ten solution of iodoform in ether. $\mathrm{He}$ injects only that amount which he feels 
can be safely left in the cavity; five to twenty grams. The trochar he uses has a cock which he closes at the completion of the injection and which thus allows the confined ether to coat thoroughly with the iodoform all the tissues with which it comes into contact as it expands. After a few moments, he opens the cock and the ether escapes, thus removing all pain from pressure.

Camphorated-thymol as an injection is recommended by some, on account of its liquefying action, if the contents of the abscess are caseous (Calvé and Gauvain, quoted by Tubby). It is prepared by mixing two parts of camphor to one of thymol, the formula determined by Ménard.

\section{GONORRHEAL INFECTION OF THE FOOT}

Next to the knee, the foot is the most frequently involved in this form of arthritis. Most writers agree that men are more often attacked than women. "Women possess a strange immunity" (Keys). But of fifty-six cases observed by C. F. Marshall, quoted by Tubby, eighteen were men and thirty-eight women.

It usually appears after the first week of the infection and may come on any time during the later stages. There are several forms in which the disease presents itself: it may include several joints and be ushered in with symptoms resembling those attending an attack of acute articular rheumatism or it may be confined to one foot and cause but slight constitutional disturbance. The differential diagnosis of the former rests on the arthritis 
remaining in the joints primarily infected and, if a new joint is attacked, in its not immediately subsiding in those first attacked; on the disproportion between the local and the general condition, the temperature, pulse, and sweating being less severe in the gonorrheal arthritis and more quickly subsiding; on the absence of effect from the use of salicylates; on the presence of a urithritis, and on the finding of gonococci in the flakes aspirated from the joint.

If but one joint is involved, and the constitutional symptoms are not pronounced, it may easily be mistaken for a tuberculous infection. The rapidity of the onset should put one on his guard, although it must be remembered that a gonorrheal arthritis may be a chronic condition. Persistent search should be made in every doubtful case for a possible origin of a gonorrheal infection.

The brunt of the attack may be on the synovial membrane, causing an exudation of fibrin, which, if not interfered with, may form bands of adhesions interfering with joint-movement and finally eroding the cartilage and causing complete ankylosis; or the periarticular structures may be the most involved, causing pronounced swelling, with marked tenosynovitis, and heat and redness. Signs of inflammation are, however, frequently absent even when the swelling is pronounced and abscess formation does not take place unless a mixed infection is present. The plantar fascia and the bursæ between the tendo Achillis and the os calcis and underneath the 
posterior tubercle of the os calcis, are frequently involved. The pain may be as severe as that accompanying articular rheumatism, but rest and immobilization are much more effective in allaying it.

A number of cases of gonorrheal arthritis have been reported in infants, complicating the same infection elsewhere.

The prognosis must depend to a great extent on the tissues involved, on the virulence of the invading organism, and on whether or not there is a mixture of other bacteria. In the mildest cases, six weeks or two months may produce a cure; while in the severer forms, six months may be necessary, and a perfect restoration may not then have been made.

Treatment. - To relieve the pain the foot should be put at rest in the most comfortable position. A posterior splint made of plaster-of-Paris, reaching from the toes to the middle of the thigh, is very satisfactory. The foot should be dressed in a thick layer of cotton-wool before the splint is applied. It may be well during the first few days to keep the patient in bed with the foot elevated. These are considered ideal cases for the application of Bier's treatment and those who have had the greatest experience with it, are enthusiastic in its power to relieve the pain and to hasten a cure. No internal medicine is indicated other than general tonics, if deemed necessary, or possibly alkalies for the urine.

The font of the infection should be attacked immediately. If the surgeon is not greatly experienced in 
genito-urinary work he had much better call in one who is, if such a one is available, rather than attempt to treat this factor himself.

Aspiration through a large-bore needle, or a small incision, a puncture, had better be performed early if there is much deposit in the joint. This relieves pain, hastens resolution, and renders material available for a bacteriological examination. The joint may be washed out with a sterile salt solution, a carbolic solution, $\mathbf{I}$ in 40 , or a bichloride solution $I$ in 5000 . In a mixed infection this is absolutely necessary. If the ankle and foot are boggy and tense and much tenosynovitis exists, several long incisions may be made and the foot placed in an antiseptic bath or put up in a wet dressing which should be kept wet. Usually, however, in this disease of the foot, immobilization and Bier's band will be all the local treatment necessary.

The danger of a resulting ankylosis and of flat-foot is always very imminent, and therefore immobilization must be interrupted by passive movements after the first few days. When the acuteness has subsided, the especially painful spots remaining, the bursæ and the tenosynovitis, may be greatly relieved by the Paquelin cautery. Bier's treatment, hot air baths, and douching with hot and cold water will often render supple, joints which seemed to be permanently limited in motion.

Vaccine treatment should be reserved for the later stages. Stock vaccine, which is put up by the large pharmaceutical houses, is quite efficient. In case of a 
mixed infection, however, it is much better to use an autogenous vaccine.

A brace which permits movements within the range of comfort is advisable, as it permits of ambulatory treatment, protects the foot from deformity, flat-foot, and preserves the movements which are not already limited. 


\title{
CHAPTER X
}

\section{OTHER AILMENTS}

\begin{abstract}
PAINFUL HEEL — METATARSALgIA - MORTON'S TOE HALLUX VALGUS - HAMMER TOE - RAYNAUD'S DISEASE - INTERMITTENT LIMPING - CHILBLAINS FROST BITE - HYPERIDROSIS - CORNS - INGROWING TOE NAIL.
\end{abstract}

PAINFUL HEEL

THIS is a condition in which the greatest pain, perhaps the only pain, is described as being in the bottom of the heel. It is most frequently found in people who have to be on their feet a great deal, as policemen, and has in consequence been called "policeman's heel." It is aggravated by use of the foot and may be entirely absent during rest, but the painful spot can always be found by digital pressure.

Examination.-In appearance the foot may be nearly, if not quite normal. Upon the patient's standing there may be slight evidence of weakness, some bulging along the inner border, and some deflection outward of the tendo Achillis; but usually any apparent abnormality is not sufficient to account for the symptoms. The dome may appear normal and, sometimes, even somewhat exaggerated. 
Palpation.-Abduction and adduction may be normal and also inversion and eversion. It is necessary to say "may be" as painful heel may exist with oncoming flat-foot. Dorsal flexion will almost always be linited to a right angle; and, in my opinion, this is the primary cause of the condition.

Etiology.- According to my theory, the painful spot or spots (there may be two) are caused by a traumatic periostitis set up by the pulling and tearing of the periosteum by the plantar tissues. From his experience, König is convinced that "calcaneum spur" in itself does not cause pain; the pain develops when the spur is injured by some trauma or becomes involved in an infectious process originating in an adjoining bursa and frequently of gonorrheal origin. The strain of a shortened heelcord, especially one not limiting dorsal flexion to more than a right angle, is felt mostly in these plantar tissues, as explained under non-deforming club-foot. Whereas this strain may result in a giving-way or stretching of these tissues, in these cases under discussion the greatest effect is in the traumatism to the periosteum at the attachment of the plantar fascia and other plantar tissues to the tubercles of the os calcis, and in a resulting chronic inflammation, a chronic periostitis. A further result, if it continues, is an excess of bony growth which may be clearly apparent in the radiograph.

Pathology.-The examination of these exostoses, when they have been removed by operation, has shown a large number to be infected with gonococci, and quite 
reasonably, these exostoses, and therefore painful heel, have been considered by many surgeons as a complication of gonorrhea. My only quarrel with this classification is that it seems to be probable that the cocci have made a nidus at these spots only after the chronic periostitis has been set up by the strain due to the shortened heel-cord. Whether or not there is a simple traumatic, or a gonorrheal infectious periostitis, the history, and especially a complement-fixation test, will help in deciding.

Treatment.- The condition of a shortened heelcord should be relieved and if a weak-foot exists it should receive appropriate treatment. The acute symptoms may sometimes be ameliorated by providing a lift to the heel which is so formed as to relieve the painful spots from direct pressure. As such a lift will also, by raising the heel, relieve some of the strain on the plantar tissues, as the range of flexion of the tibia on the plane of the ground in walking is thereby increased, further relief may be obtained.

Operation.- In the hands of some surgeons, excision of the exostoses gives splendid results. They should be reached from a lateral, longitudinal incision on the external aspect, so as to avoid a scar in the tread of the heel. Doubtless the rest in the plaster-of-Paris dressing after the operation is of material benefit, too.

Vaccine treatment in cases where a complement-fixation test has been positive may be tried. 


\section{METATARSALGIA}

Metatarsalgia is a painful condition in which the part of the dome formed by the metatarsal bones, the transverse arch, is weakened, lowered, and sometimes obliterated. Normally this part of the foot is very flexible, although any one direction of movement is greatly limited. This flexibility is essential to proper poise and

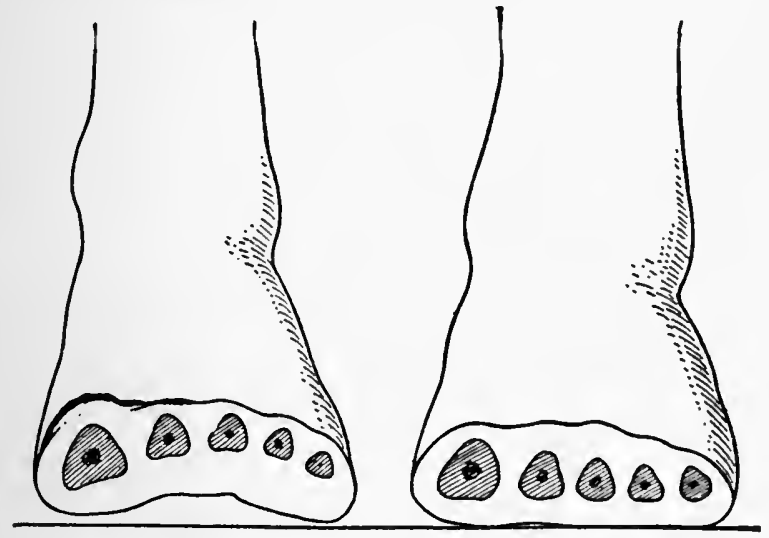

Figs. 85-86. Transuerse Section of the Forefoot

Fig. 85 shows the normal position of the metatarsals and Fig. 86 their abnormal position when their arch is flattened.

balance. If for any reason the muscles controlling the metatarsals become weak, the ligaments will stretch, the arch be lowered, and movement between these bones be lost, together with the finer movements of the toes.

Etiology.-Although severe traumatism may destroy this arch, as from falls from a height or by the falling of heavy weights upon this part of the foot, the 
condition under consideration is the result of weakness. Usually it is the effect of non-use. Many shoes are made so as effectually to immobilize the forefoot.

Metatarsalgia is more common among women than men. It is rare during childhood.

Pain.- The pain is of an aching character, brought on by use of the foot and sometimes remaining indefinitely during rest. When great weakness exists, the pain will be excited whether the patient walks in a broad-toe shoe or even bare-footed. The pain is usually confined to the forefoot.

Examination.- If the condition is of long standing, calluses will be found under each of the metatarsal heads, as a rule; although they may be only under the second, third, and fourth. In the former case, a right angled contraction of the heel-cord will be present. The forefoot is broader than normal and usually appears thinner, depressions sometimes being present between the metatarsals, as it is seen between the metacarpals in muscular atrophy of the hand. On the other hand, the forefoot may be swollen and somewhat inflamed and, according to Tubby, lead to a diagnosis of gout or rheumatism. In examining the shoes, it may be found, as pointed out by Goldthwait, that the sole is concave from side to side so that the first and fifth metatarsals are held on a higher plane than the others, and may also be concave antero-posteriorly so that the phalanges are held extended in a position to force down the heads of the metatarsals. The shape of the sides of the shoes 
will have a very great determining influence on the position which the bones will take when the arch is materially weakened.

Treatment.-This is sometimes so easy and so successful that one case will bring renown to the surgeon among the patient's friends. In others the greatest patience is demanded and many therapeutic measures will be tried before a cure is obtained. The object is to strengthen the arch, and to keep it in the position of strength while this is being accomplished. When a shortened gastrocnemius or a weak-foot exists, appropriate measures must be taken for their treatment. Exercises which will strengthen the small muscles of the forefoot are indicated: picking up objects, as a marble (Osgood); and voluntary attempts at abducting, flexing, and extending the toes. It is best to be very specific in prescribing the time for these exercises, as five minutes every morning and evening, with instructions to move the toes, while the shoes are on, at the stroke of each hour throughout the day.

Support may be had in a number of ways: if a brace is to be made for the antero-posterior arch, it can be extended forward and a convexity made on its upper surface beneath the anterior arch. If the bases of the metatarsal bones are grasped in the hand and slightly compressed, their heads slightly separate and they may be easily made to assume the arched position: a piece of adhesive strapping about this part, acting as does the hand, may frequently be sufficient support. A piece 
of felt, varying in size according to necessity, may be placed under this arch and retained by adhesive plaster. A leather insole, exactly fitting in the shoe so that it can not vary its position, may have secured to it a piece of felt or soft rubber, which will support this arch when the foot is weight-bearing. When the exact location for this pad, on the insole, has been determined, a cot may be secured, opened at one end; and this will admit of fresh padding being inserted as may become necessary. The amount of padding which can be tolerated and which will afford relief, varies to a remarkable extent. I have had patients made quite comfortable, and able to travel abroad, visiting art galleries and being on their feet a great deal, with a piece of soft rubber the size of their own forefinger supporting this arch; while in other cases of apparently the same degree of weakness and deformity, a piece of soft felt the size of a small pencil, could not be tolerated. Sometimes, by having the shank of the sole of the shoe made very narrow, the lacing over the waist can be drawn so snugly as to effect what strapping accomplishes.

\section{MORTON'S TOE}

Morton, of Philadelphia, was the first one to describe this condition. It is often confused with anterior metatarsalgia, but has a distinct pathology. The two conditions may be present at the same time.

Etiology.- Morton's toe is a neuritis, caused by nerve pressure by the head of the fourth metatarsal. 
It may be pressure on one of the larger branches of the external plantar nerve or on the nerve formed by communicating branches of the external and internal, or on some one of the filaments from the plantar, or from the anterior tibial. The pressure is usually between the head of the fourth metatarsal and the base of the first phalanx of the fifth toe, or it is an intermetatarsal pressure between the third and the fourth. Whitman has drawn attention to the similarity in the character of this pain to that elicited in the hand by compressing laterally the metacarpals while they are held in the same plane, and are not allowed to form the carpal arch. Tubby has twice found evidence of true nerve inflammation.

Diagnosis. - The pain in this condition is characteristic: it comes on suddenly, sometimes from a slight traumatism; is sharp, lancinating, and defined to the fourth toe, extending down to its tip and also, frequently, up the leg, following the nerve's course. The sufferer may be compelled to remove the shoe immediately, and will instinctively rub the forefoot, compress and relax it, and flex and extend the toes. It may be possible to replace the shoe in a few moments and be comfortable until another traumatism, perhaps some particular poise or step, excites it again. When the attacks become frequent, and a chronic neuritis results, the slightest pressure causes pain so that walking, especially with a shoe, is almost impossible.

Examination.-A callus may be found beneath the 
fourth metatarsal and its head may be distinctly beneath its normal plane. The base of the fifth metatarsal may be prominent and its shaft incline inwards toward the fourth, while the little toe is directed outward, so that the base of the first phalanx is directed inward toward the head of the fourth metatarsal.

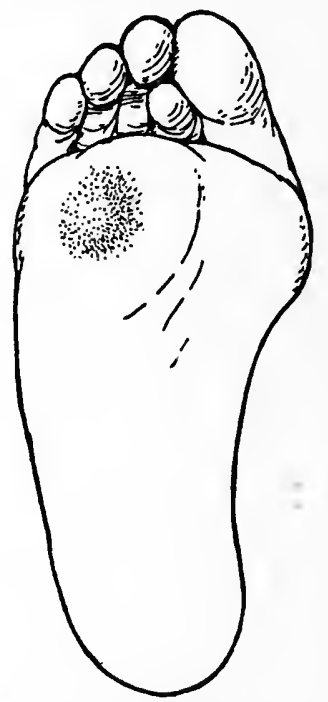

Fig. 87. Callus in Morton's Toe

The situation of this callus is very characteristic. It lies beneath the head of the fourth metatarsal bone. The calluses resulting from a shortened heel-cord are usually five in number, lying beneath the heads of the five metatarsals. There is present in the above illustration a hallux valgus and also a hammer toe.

Treatment.-Sometimes a pad, carefully shaped and applied to restore the transverse arch, and strapping to separate the bones may be successful. When the radiograph shows an undoubted impinging on the head of 
the fourth metatarsal by the third metatarsal or by the first phalanx of the little toe, an excision of the fourth metatarsal head may be undertaken. It is a simple operation and, aside from a slight retraction of the fourth toe, is not followed by any bad results. Resection has been done of other metatarsal heads with reported cures. On the other hand it is easy to be deceived by the radiograph and to give an excellent prognosis, only to reap disappointment, an unfortunate experience which I once had. It is now my custom to withhold operation until all other means of cure have been exhausted.

An operation has been devised and practiced by Forbes which consists of a transplantation of the extensor tendon of the fourth toe into the head of the metatarsal.

\section{HALLUX VALGUS}

This is a condition in which the great toe is directed in toward the middle line of the foot and toward the other toes, and the first metatarsal-phalangeal joint is made prominent on the foot's inner border.

Etiology.-Its most frequent cause is the crowding together of the toes in a narrow-toed shoe. After the deformity has started, a short shoe will increase it by pressing backward against the tip of the toe and crowding the head of the first metatarsal outward from the middle line of the foot. The extent of the deformity may vary from a slightly abnormal inclination to almost a right angle with the other toes, so that the great 
toe may lie upon and across the others. A bursa, called here a bunion, develops on the side of the joint where it presses against the side of the shoe.

The loss of the normal function of this toe precludes proper walking and the prevention and cure of this deformity is therefore of great importance. Shoes likely to cause it should be unhesitatingly condemned even if otherwise perfect.

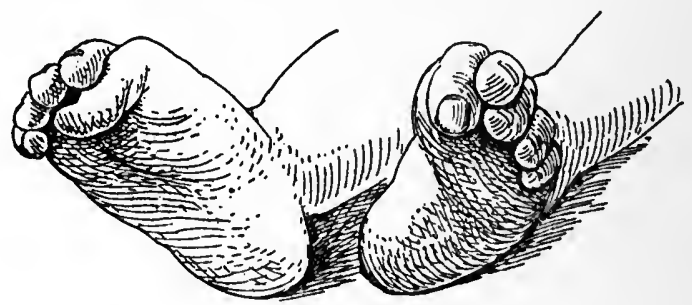

Fig. 88. Hallux Valgus

The great-toe is turned inward toward the middle line of the foot. The cause in this case was paralysis. When due to faulty foot-wear the deformed toe usually lies above the other toes and the metatarsal head is made very prominent by pressure outward, toward the other foot, through the phalanges.

Treatment.- When the deformity is slight and the toe can easily be brought by passive movement to its normal position, exercises, manipulations, and correct foot-wear may be all that is needed. But the detail of such treatment must receive conscientious attention to be effective. At night a brace may be worn to retain the toe in a straight position. A piece of steel spring, secured along the inner border of the foot by straps and having a strap to hold the toe against it, will be all that is demanded. 
Various braces have been made designed for wear in the shoe. Perhaps the most popular device is a stall made by inserting a small piece of steel through an insole so that it will press against the side of the toe and hold it in a straight position. An insole is obtained which fits the shoe accurately, the foot is placed upon it, and the cleft marked between the first and second toes. A piece of light steel (tin is satisfactory) is cut so as to be one-half inch longer than double the depth of the toe, the height of the top of the toe from the ground, and of a width equal to the length of the first phalanx. This is doubled upon itself, on its long diameter, and the ends for the last quarter of their lengths bent at right angles to make flanges. The end of the contrivance will now have the shape of an inverted $T$ with the stem having two layers and the flanges one each. This stall is then pressed through a slot made in the sole at the place marked as the site of the cleft, the flanges coming against the bottom of the inner-sole and holding the stall in place. The stall had better be covered with a little padding which may be secured by adhesive plaster. If the stall is not high enough the toe will at times get above it and cause severe pain. This method is only practical in moderately deformed cases, where the deformity is easily corrected by manipulation.

Wedges made of cotton may be used to separate the first and second toes and together with other treatment, especially exercises, will be of assistance. 
Operation.- When the deformity is of long standing, bony changes and shortening of the soft structures on the inner side of the joint will make an operation imperative. This is best done by a curved incision around the bursa with the base downward; careful dissection of the flap off from the bursa, a second in-

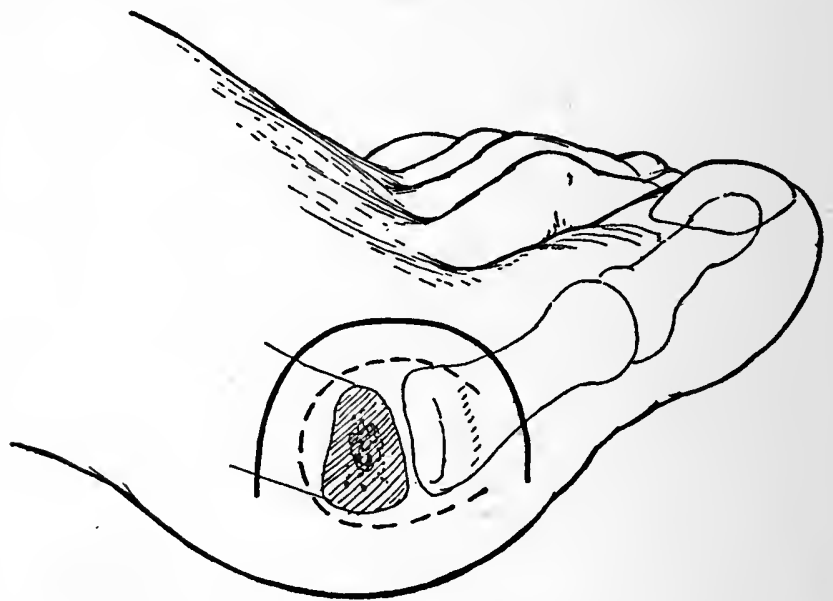

Fig. 89. Operation for Hallux Valgus

The solid line represents the skin incision, the interrupted line the incision for the flap containing the bursa, which is turned in between the metatarsal and the phalanx, after removal of the head of the former.

cision, forming a flap with its base forward and including the bursa; removal of the head of the metatarsal in a line not directly at right angles to the long axis of the bone, but extending outward and slightly forward; making the end of the bone smooth with the rongeur; placing the bursa in the resected joints and securing with 
a suture; closing the wound and applying a plaster-ofParis dressing with the great toe in the straight or slightly abducted position. A movable joint may be expected as a result.

Objection to the operation has been made on the score that removal of the head of the first metatarsal takes away an important support of the forefoot. However, no unfavorable results have been reported, and, on the other hand, C. H. Mayo reports (Annals of Surgery) sixty-five operations, all of which were most satisfactory.

\section{HAM:MER-TOE}

When one of the smaller toes has become flexed at its first inter-phalangeal joint, so that this joint stands up prominently above the plane of the other toes, and is not extensible even by passive movement, it is called a hammer-toe.

Etiology.-It is caused, in the last instance at least, by the toe's being retained in this position continuously by the crowding together of the other. The chief reason for its exciting any complaint is that the summit of the flexed joint is subject to pressure against the shoe leather and a painful corn is usually the result.

Treatment.-If not of too long standing, the prescribing of proper foot-wear and of exercises for the toes, may relieve the condition. The upper of the shoe over the toes should be of sufficient room to avoid pressure on the joint; a soft leather will be less irritating than a stiff leather. A simple arrangement may be 
made to hold the toe extended by strapping it down to an inner sole, the straps running through slots opposite the cleft on either side of the deformed toe.

Operation.- In cases of long standing, however, success is not likely to be achieved by any means short of an operation. Simple division of the contracted soft tissues, followed by retention of the toe in the straight position for two weeks, will be all that is needed, unless there have been marked bony changes. The contracted structures are usually the lateral ligaments and sometimes the plantar ligament. All three may be divided through a small puncture on one side. If the joint is much altered in shape the extension made possible by dividing the tissues on the flexor side can not be expected to remain permanent. Under such circumstances a resection of the joint is better when an operation has been undertaken.

\section{RAYNAUD'S DISEASE}

Usually a symptom of some disease affecting the nervous system, Raynaud's disease may, rarely, exist without any such connection. It may affect any of the extremities or even other parts of the body. It is most frequently seen in middle life, attacking women more often than men. It is not always symmetrical, although this was at one time considered one of its characteristics. If symmetry is present it is the more easily diagnosed. There are three stages, the first and second coming in paroxysms; local syncopy, local cyanosis, and local 
gangrene. The disease may present the symptoms of the second stage without those of the first having been present or at least without the symptoms having been recognized.

In the stage of syncopy, caused by spasm of the arterioles, the part, usually the plantar surface of the toes, becomes white and cold and there is present a disturbance of sensation. It may be brought on by exposure to a low temperature, not necessarily a very cold temperature, or by walking or standing. It is attended with pain which may not be severe, but is, in some cases, excruciating. Warmth and rest may restore the part to its normal condition. The disease may express itself only in these attacks of the first stage and may not progress to the second stage. At any time it may be arrested and not return for months or even years.

The second stage, that of local asphyxia, usually follows the first. It is caused by spasm of the venules. The part becomes blue or purple, cold and edematous. Pain may vary as in the first stage.

The third stage, which does not follow in every case, is that of gangrene. This results in a mummification of the part.

Treatment.- Anything which will help to correct the condition of the vasomotor system will help to prevent the attacks and to produce a cure. General hygienic treatment, tonics, and equable temperature are of great value. When gangrene is present, care should 
be taken against infection. The line of demarkation should be awaited before active interference is undertaken.

\section{MYASTHENIA ANGIO-SCLEROTICA. INTERMITTENT}

\section{CLAUDICATIO. INTERMITTENT LIMPING}

This is a rare condition in which a severe, spontaneous, cramp-like pain occurs in the calf while walking. A moment's halt relieves the pain, walking is resumed, and the attack returns. There is present a circulatory disturbance which causes a feeling of coldness in the foot accompanied by a blanching of the skin. Lovett reports the absence of pulse in the dorsalis pedis and in the posterior tibial arteries in two of his cases.

Treatment.-Codein, given in small doses three times a day, has been highly reported. If syphilis, diabetes, or chronic alcoholism exists appropriate treatment may alleviate the local condition.

\section{PERFORATING ULCER OF THE FOOT}

This is a neuropathic ulcer occurring in the sole, usually in the metatarsal region, painless, and showing no tendency to heal. Other stigmata of the nervous condition underlying the ulcer may be also found in other parts of the body, such as the condition of the skin and nails of the hands. It may be associated with either a peripheral neuritis or with some central lesion. The ulcer is usually neglected on account of its analgesic condition 
and may be an eighth or a quarter of an inch in diameter before treatment is sought and may reach to and involve the bone. The callous formation about the ulcer may lead to the diagnosis of an infected corn.

Treatment. - The underlying condition should be sought for and appropriately treated. Local treatment consists in thoroughly cleansing the wound, using the curette freely, and lightly packing and dressing. A mildly stimulating antiseptic may be of value. Balsam of Peru mixed with castor oil is to be recommended.

\section{PERNIO. CHILBLAINS}

This is a condition of disturbance of the circulation to a part and results from prolonged exposure to moderate cold. The skin assumes a purplish color and there may be edema. The subjective symptoms are a burning pain with itching. A foot which is partly or completely paralyzed is very subject to chilblains. Anemia is a predisposing factor. According to Wright there is present in people who suffer from this condition a deficiency of the calcium salts.

Treatment.-This consists in the prevention of exposure to cold. Woolen stockings should be worn in cold weather. The burning and itching may be relieved by the application of cooling lotions, as a fifty per cent. solution of alcohol. If the skin becomes broken from scratching, infection must be guarded against. Internal treatment should be directed against anemia if such exists. Calcium is reported to be a specific and 
may be given as the lactate or the chloride. It has been recommended that its administration be commenced at the beginning of cold weather and continued for several weeks in cases of recurring chilblains.

\section{CONGELATION. FROST-BITE}

This is the effect of a prolonged exposure to a severe cold. The diagnosis is not difficult.

Treatment.- The generally accepted plan of treatment is the gradual restoration of the circulation by massage with snow or cold water. It is the opinion of some surgeons that a too sudden raising of the temperature results in a paralysis of the muscular coats of the vessels. Doubtless the slow restoration of the circulation is much less painful, but it is not satisfactorily. proved that gangrene is any the less likely to occur. The foot should not be kept continually moist for days, but after thirty-six or forty-eight hours should be dried, powdered with boric acid, wrapped in wool, and placed in an elevated position. If gangrene sets in, the line of demarkation should be awaited before the knife is used. Bullæ should be treated as they are in burns.

\section{HYPERIDROSIS. EXCESSIVE SWEATING}

When this condition attacks the feet it may prove to be a most disagreeable and troublesome affection. Not only the stockings but the shoe-leather as well become damp and a pungent and penetrating odor, "bromidrosis," is created, due to bacterial growth. There is 
present a functional disease of the sweat glands of the feet, the cause of which, except that it is probably of nervous origin, has not been determined.

Treatment. - Mild cases may demand nothing more than a change of stockings twice a day and the application of a powder made up of equal parts of boric acid and salicylic acid. If the shoes are damp when the stockings are changed another pair should be put on. When only the soles of the shoes are-damp, the use of cork insoles may answer the purpose, a fresh pair being inserted whenever the stockings are changed. It will generally be found that these patients are in the habit of frequently bathing their feet in very hot water, their object being to keep their feet odorless. It is much better to bathe the feet in cold water or better still to douche them with alternately hot and cold water, then dry, rub with alcohol and powder. In severe cases Hebra used an ointment made of lead plaster and olive oil. This he applied on strips of linen after the feet had been thoroughly washed. This dressing is not disturbed for twenty-four hours when fresh ointment is applied without, however, again washing the feet. If necessary this procedure is kept up for a week or even two when the epidermis comes off in large plates. The feet are then washed and treated with boric acid powder. Careful strapping of the feet with zinc oxid plaster is much easier to apply and is very gratifying in its results.

The general condition of the patient should receive 
attention at the same time as the feet, as most of these patients will be found to be run down and more or less anemic and nervous.

\section{ERYTHROMELALGIA}

A chronic disease in which there is a painful congestion of the feet and to a less extent of the hands. It is at first limited to the heel, or perhaps to the forefoot and occurs only at night but finally extends over the entire plantar surface and is present night and day. When it has progressed to this stage walking or even standing becomes very painful.

PLANTAR NEURALGIA

Erythromelalgia is considered by Dana as a form of plantar neuralgia. He states that in rare cases the pain of sciatica is limited to the plantar nerves and is accompanied by anesthesia and paresthesia of this region. It may be present in insular sclerosis, tabes dorsalis, and myelitis.

\section{CLAVUS. CORNS}

The skin is being constantly renewed by the multiplication of its deeper cells and the shedding of its external ones. The new cells push the older ones upward into the outermost layer of the epidermis, the horny layer or stratum corneum, whence they are cast off. If pressure is exerted against an area, these cells are not only prevented from escaping ahead of those pushing 
them outward; but, if the pressure continues, they are crowded back into a compact mass. The cells pile up and spread out beneath the point of pressure; and in consequence a cone-shaped mass is formed, the apex being in the deep layer and the base on the external surface. It is thus that a corn is formed. Its apex, pressed in among the papillæ which are extremely abundant in nerves, causes the pain which is an accompaniment of all corns. When this external pressure is made by an adjoining toe the outer layer of the corn is kept moist and thus a "soft corn" is formed. If the pressure is intermittent as is more frequently the case in the sole of the foot, calluses are produced. These, while causing the piling up of the outer layer, do not so confine the cells as to form the distinct hard cone seen in the veritable corn. In the formation, therefore, a corn is first a callus and later, as the pressure continues, the cells become more and more compact so that a dense center column, the core, is formed.

A corn is always the result of pressure and much valuable information in the diagnosing of foot-deformities can be obtained by noting the location of a corn. It is quite essential to bear in mind that although the patient's foot may be in otherwise perfect physical condition, and the pressure may be caused by an ill-designed shoe; on the other hand the shoe may be designed for a perfect foot and be of a correct size; but owing to some faulty condition of the foot, as will be the case if it is held in abduction, the skin will be pressed un- 
duly against the leather. Spasm of the peronei will so abduct the fore-foot that pressure will be exerted opposite the head of the fifth metatarsal regardless of what shape shoe is worn. A short shoe with a narrow toe cannot fail to crowd one or more of the toes into flexion causing the skin over the proximal interphalangeal joint to press against the leather and produce a corn. Thus a hammer-toe frequently has a corn over this joint. If a hallux valgus is present a short toe will press the toe backward and outward so as to crowd the head of the first metatarsal against the leather of the shoe. As a hallux valgus interrupts the symmetry of the inner line of the foot and shoes are not usually made to accommodate this bulging joint, there is pressure here even in a shoe of ample length and a corn in this condition is almost invariably present.

In some cases it may be noted that the skin seems to produce corns with extraordinary facility and will not tolerate pressure which would cause no disturbance in other cases.

In pityriasis rubra pilaris corns are easily formed.

Diagnosis. - A small perforating ulcer has a callus about it and may easily be mistaken for a corn, a mistake which can be avoided if this fact is kept in mind during the examination.

Treatment. - It hardly need be pointed out that the removal of the cause is essential. We know that the cause is the pressure of the skin against the leather and the removal of pressure may be obtained by stretch- 
ing the leather or cutting it away, proceedings frequently resorted to by the patients themselves. The rings of felt sold in the drug-stores for this purpose are generally efficient. Some shoe-makers, when hallux valgus is present, make a ring of leather with wings or extensions extending forward and backward, which when placed in the shoe not only prevent pressure but hide the deformity quite effectually. All of these measures are but palliative: they recognize pressure as the cause, but do not remove the cause of the pressure.

If an on-coming deformity, such as a beginning flatfoot or hallux valgus, is found or if a deformity already exists, appropriate treatment should be directed toward the correction of such. The shoes must be changed if they are too short or too narrow or if they hold the forefoot abnormally abducted or adducted.

To remove the corn, it should first be softened. This may be done by soaking the foot in a hot bath for twenty minutes and then drying the foot and applying salicylic acid. Salicylic acid acts chemically upon the callus, softening it so as to facilitate its removal. It may be put up in flexible collodion, five grains to the ounce, and painted on with a camel's hair brush; or it may be applied in the form of an ointment made up of lanoline and vaseline, five parts of each and one part of salicylic acid. In ten or twelve hours the softened callus appears as a white mass and can be picked off. It may be necessary to repeat the treatment, perhaps two or three times before the last vestige of the corn is removed. Naturally, 
if the original pressure is operative after each treatment, the corn will return and the above procedure will have to be repeated indefinitely.

There are two corns which deserve special mention: the soft corn and the corn over an hallux valgus. The soft corn can be effectually treated by the process as above described and then by placing a pledget of cotton between the toes. A broad-toed shoe is essential for a cure. The corn accompanying a hallux valgus will often have a bursa developed beneath it. This corn is more subject to inflammation than any other and it is frequently called a bunion. The treatment quite commonly resorted to, of paring it with a knife, may result in an infection and a serious condition thereby be produced. When infection is present half-way measures must not be tolerated. The patient must remain indoors to the end that appropriate treatment can be carried out. It may. be sufficient to apply hot boric acid dressings, under oiled silk, the dressing being large enough to cover the entire forefoot; or more radical procedure may be demanded, such as opening and draining. As the bursa beneath this corn communicates with the metatarsal-phalangeal joint, neglect or improper treatment may prove to be a rather serious matter.

ONYCHIA. PARONYCHIA.' INGROWING TOE-NAIL

This is an inflammation of the matrix and the soft parts about the nail. Paronychia of the toes is most frequently found on the large toe and is commonly called 
ingrowing toe nail. It is caused by the pressure of the shoe against the side of the toe, forcing the soft parts against the nail which may have an abnormally sharp edge, usually from careless trimming. The soft parts become inflamed and swollen and a painful condition exists.

Treatment.-This must depend upon the severity

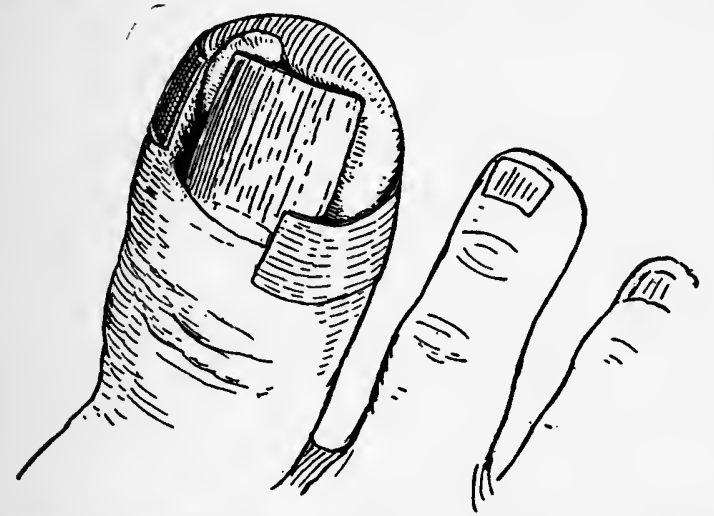

Fig. 90. Conservative Treatment of Ingrowing Toe-Nail

The irritation from the nail is relieved by a pledget of cotton and the exuberant tissue is drawn away by a strip of adhesive plaster.

of the case and the control one can expect to have over the patient. In dispensary patients radical measures are the most satisfactory. When time is not of importance, operation is seldom necessary. Moreover, in private practice this condition is apt to be called to the surgeon's attention in its early stages. Conservative treatment consists first in removing all pressure such as may be caused by a pointed or narrow shoe or even by a narrow or short-toed stocking, and then in removing the irrita- 


\section{DISEASES AND DEFORMITIES OF THE FOOT}

tion caused by the pressure of the side of the nail. This last may be done by pressing a little piece of cotton with a probe or an orange wood stick or a pointed match under the edge of the nail and between it and the swollen parts. The soft parts may be further relieved by drawing them away from the nail with a narrow strip of adhesive plaster encircling the toe. When conservative treatment is undertaken the patient should be instructed to trim the nail straight across and not to cut down the corner or inner edge.

Radical treatment consists in the removal of the sharp edge of the nail. About a third of the nail should be taken off together with that part of the matrix and the swollen soft part. It can readily be done under a local anesthetic. A rubber tube is first fastened about the base of the toe and cocaine, if that is used, is injected around the nail. The first thrust of the needle should cause all the pain there is to bear. The incision should be elliptical, including all that is to be removed. Horse hair sutures are the best for closing the wound. The edges will not be brought close together; but there will be less surface to granulate, the time of healing will be shortened; and the co-aptation of the V-shaped depression left in the soft parts by the removal of the exuberant tissue will the better insure a rapid cure.

Should the toe be acutely inflamed and very painful it may be better to defer operation until the condition has been rendered less acute by a few days' treatment with rest and wet dressings. 
CALlUS. CALLOSITES

Callus will appear wherever the skin is subjected to constant irritation. When on the feet it is due to deformities, faulty mechanism in the use of the feet, irritation from foot-wear, or the presence of one of two diseases. If the callus is of long standing, a bursa may be developed beneath it, as is frequently found beneath the callus over the cuboid in a much used talipes equinus varus.

Frequently the location of the callus and its extent and hardness are guides to the condition of the foot. Calluses under the heads of the five metatarsals are evidence of a complete lowering of the transverse arch and will be found in non-deforming club-foot of long standing and also in cases of anterior metatarsalgia. If the callus is under the head of only one metatarsal, usually the fourth or third, the condition found in Morton's toe is present.

There are two diseases which may be accompanied by calluses of the soles; ichthyosis and pityriasis rubra pilaris.

Treatment.- The cause of the irritation must be determined and dealt with accordingly. The callus may be removed by the treatment laid down for corns, or it may be left to desquamate, as it will if the cause of its inception is eradicated. 
276 DISEASES AND DEFORMITIES OF THE FOOT

\section{PAINFUL SOLES}

Pain in the sole of the foot may be present with any of the following conditions: deformities, new growths, contractions, scar tissue, absorption of fat from pressure or following prolonged illness, gout, rheumatism, arthritis deformans, inflammatory arthritis, as tuberculous or gonorrheal, Raynaud's disease, erythromelalgia, plantar neuralgia. 


\section{CHAPTER XI}

\section{FOOT-APPAREL}

IT is quite generally admitted that many deformities of the foot are due to faulty clothing. Some French surgeons, to be sure, have recently taken pains to discount this opinion, claiming that some pathological condition of muscles, nerve, or joint is the primary etiological factor and that the shape of the foot-clothing is of but secondary importance.

"Without wishing to ignore the rôle played by malconstructed shoes in the production of deformities of the toes, it seems to us that such causes ought to be considered as accessories; the rôle by far the most important, it seems to us, appertains to general causes, among which chronic rheumatism ought to have first place." *

While this may be true in some cases, we do not believe that most surgeons agree that it is so in the majority of the deformities of the foot.

Stockings. - A stocking so short as to prevent full extension of the toes or so pointed as to cause adduction of the great toe and of the two outer toes, would not

* Les Difformités des Orteils. Kirmisson et Baillene. Revue D'Orthopédie. Ier Mars, Igr3. 
cause a permanent deformity were it worn but a few hours and the muscles and joints properly exercised when freed of the restraining influence of the stocking. The gravity of the restraint of a confining shoe or stocking is due to the prolonged time of its effect. Improper walking and standing have so trained many people to use the foot as though it were one solid mass that upon removing the shoes and stockings they will walk the little that is necessary, bare-footed or in bed-room slippers, go to bed, and dress in the morning without having moved the toes or the smaller joints of the foot to even the smallest extent.

As stockings and socks are'made of flexible and elastic material, their influence is limited to that of compressing the more movable joints, especially the toes. Digitated stockings have been made but were never very, popular. "Rights and lefts" are on the market and are to be recommended if one does not try to get too good a fit and thereby get a stocking that is too short. The ordinary stocking, as long as it is not too pointed and not too short, is beyond criticism. Whether it should be made of silk, wool, or cotton will depend upon the circulation of the foot.

Shoes.- A great many attempts have been made to produce a perfect shoe. Shoemakers have been at no little pains to turn out a confection of their art which would meet the scientific requirements as they have understood them. The results have been encouraging although they have been far from perfect. As soon as 
it has been definitely decided just what constitutes a perfect shoe, a long step ahead will have been taken. When the surgeons are finally in accord on this subject it is not probable that Dame Fashion will be able to ignore the dicta as to shoe-construction as laid down by Master Science. She has been forced to bow to so many laws of hygiene that we hope she will allow her toes to be stepped on.

As it is, we should feel pleased at the advance that has been made in the styles of shoes sold in the markets to-day. Orthopedic, Common Sense, and various other named shoes have a constantly increasing sale, showing the desire of the laity to treat their feet with becoming respect and not to be too much the slaves of the passing whims of fashion. Some of these shoes have the forefoot so adducted that the outer border of the soles form almost a quadrant of a circle; some have simply a broad, square toe; some an extension or Thomas heel; some a wedge-shaped heel; and some have stiffening of one sort or another under the dome. Most of these modifications have their therapeutic value, but, for the normal foot, some of them are likely to prove to be injurious.

The first requirement of a shoe for a normal foot is that it must permit the free functionating of the foot. This is of less importance if the foot is not to functionate while in the shoe; but as most shoes are used for standing and walking, we need not consider the shoe worn only while the foot is at rest. 
Heels.-First as to the heel; a high-heel is injurious only when being used. The greatest objection to the high-heel is that it makes it so difficult to walk on the toes. Most of them are found on narrow, pointed shoes which are of themselves objectionable. If high-heels are worn constantly they may also be a contributing factor to non-deforming club-foot. Of the two most popular forms of high-heels, that which has its supporting surface the more anterior is the better, the French, rather than

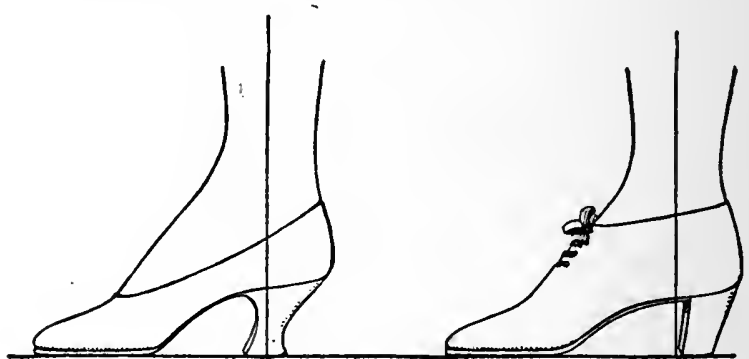

Figs. 9I-92. The French and Cuban Heels

The heel of a shoe should have its base in the plane of gravity to give the greatest support. If it is behind this plane it may be a contributing cause of weakness of the ligaments and muscles.

the Cuban heel. The ideal heel is not higher than the sole, has straight sides and extends well forward, to the plane of the anterior border of the internal malleolus, thus giving a firm and extensive support.

Toe.-The main features to be sought in the toe of the shoe are, that the toes have room to extend and to flex; that the great toe has room for abduction and rotation; that the metatarsals are not cramped; and that the forefoot as a whole is not held in abduction. 
As a cross section through the heads of the metatarsals has its thickest part over the head of the first, the leather covering the toe of the shoe should not offer the most room to the center of the foot, to the middle metatarsal, as is so often the case; but the highest part of the toe of the shoe should be over the great toe. A last which does not take this into consideration will exert

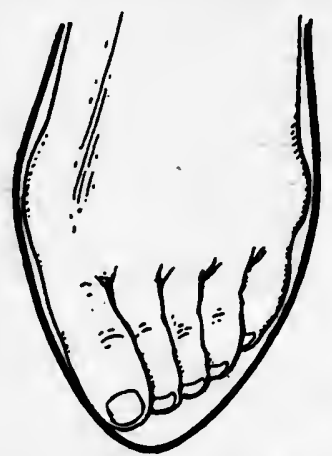

Fig. 93. A Faulty Shoe-Toe

Illustrating the evil effect of the inner border of the shoe, at the toe, turning inward too sharply. This shoe does not increase the deformity of the great toe by pressing it directly inward, but by pressing against the tip in a direction backward and inward. This force is felt at the first metatarsal-phalangeal joint in a direction outward and backward; the direction best calculated to produce a marked hallux valgus.

an adducting force on the large toe and prevent its abduction and rotation, its normal movements.

In the normal foot, the line connecting the middle of the tip of the great toe with the center of the first metatarsal-phalangeal joint will, if continued backwards, pass over the center of the heel. If the inner side of the great toe, when in the shoe, can for its entire length form a 
straight line with the inner border of the heel, this requirement is met. As the anterior part of the heel of the shoe is frequently not at a right angle to the long diameter of the shoe, care must be taken that one is not thereby deceived.
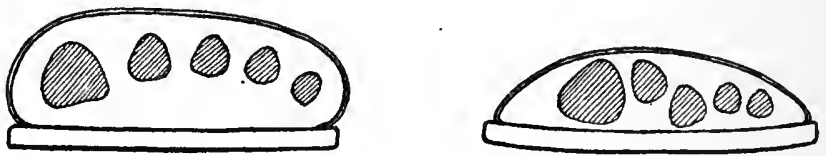

Figs. 94-95. The Toe of the Shoe

The sole should be sufficiently stout to prevent its being curled up at the sides. The space within should be ample for the free functionating of the metatarsals and the phalanges. The figure on the right shows a badly fashioned shoe-toe: the sole curls up and the greatest depth of the inside measurement is in the center, thus crowding the bones together and forcing the third metatarsal onto a lower level.

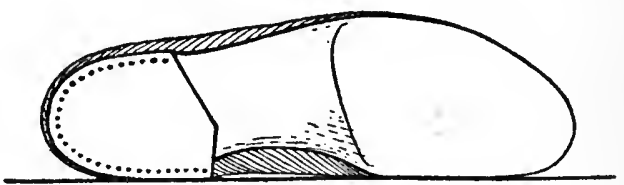

Fig. 96. An Excellent Shoe

The heel extends forward on the inner side and also further inwards than is usual. Secure support is thus given in the plane of gravity and eversion is prevented. There is ample room to permit of abduction of the great-toe and also to provide for the alterations in the shape of the dome of the foot while the foot is functionating. It is not unsightly.

The breadth of the toe should be such that the toes can be fully extended and flexed as easily as they could be were one wearing sandals. The interference with this movement is the greatest cause of anterior metatarsalgia.

Shank. - The waist of the shoe should fit snugly and 
should not be so far forward, there should not be so short a vamp, as to limit movement at the medio-tarsal joint. The shank must be narrow, for if wide it will prevent the leather along the inner side from being laced snugly up against the longitudinal arch. The shank usually contains a steel spring which undoubtedly aids in preserving the shape of the shoe; but if walking is properly done and if the heel is not too high, it is unnecessary. Providing it is not so long as to obstruct movement at the medio-tarsal joint, however, there can be but little objection to it.

The importance of the distance of the part of the sole beneath the first metatarsal-phalangeal joint and the center of the heel, has been pointed out by Sampson. If the inner border of the sole begins to curve too soon toward the shank, the upper at this point will unduly press against the head of the first metatarsal and will not only operate against the normal movement of the great toe, abduction (away from the middle line of the foot) and rotation, but will exert an abducting force on the forefoot.

Upper.-The uppers may be low, the "shoe" of the English, or high, the English "boot." The low shoe offers more freedom to ankle movements and the close fitting high tops may be the cause of a certain amount of weakness. If high shoes are worn we should advise that under ordinary circumstances the uppers be soft, not too stiff and unyielding, and never reennforced with whalebone or other material unless assuredly indicated by some 
abnormal condition. A lace fastening is always preferable to any other kind.

Rocker-sole. - It remains to say a word regarding the rocker-sole. It is the claim of shoemakers that if the sole is made to lie flat on the ground, especially with a low heel, it will be turned up at the toe after it has been worn a short while and not be so presentable as a shoe which was made that way in the first place. To a great extent this is true, due to the habit of heel-and-toe walking. If walking consisted in rolling onto the heel and
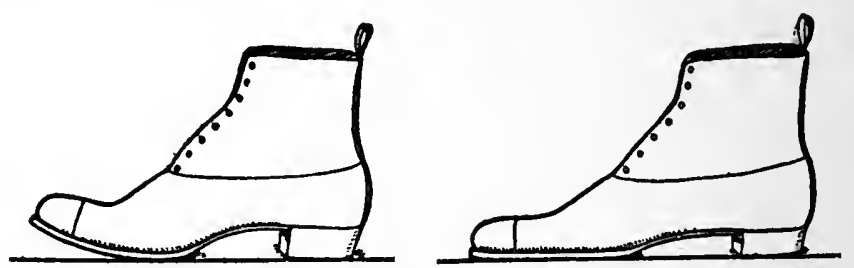

Figs. 97-98. Rocker-Sole aNd Flat-Sole Shoe

The former is the shape assumed by any shoe, with a low heel, which is worn by a heel-walker. With such a shoe it is impossible to walk properly.

off the toe, the rocker-sole would be our choice; but for the normal manner of walking as we conceive it, the toes should bear the same relation to the rest of the foot while the shoe is on that they do in the normal barefoot, whether walking, standing, or resting.

Fit.- In the consideration of shoes, it is well to remember not only that the distance across the metatarsals increases during standing, due to the abduction of the first metatarsal and to a slight lowering of the metatarsal arch; but that there is a lowering of the dome as a whole. 
This may be demonstrated by marking the position of the tubercle of the scaphoid and observing its changed

Figs. 99-ioo. Alterations in the Length of the Foot During REST AND WEIGHT-BEARING

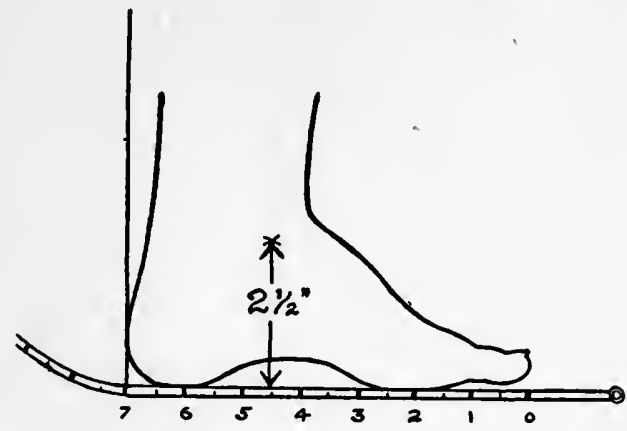

During rest: the foot is represented as 7 inches long and the scaphoid-tubercle (imperfectly marked) as $2^{1} / 2$ inches above the ground.

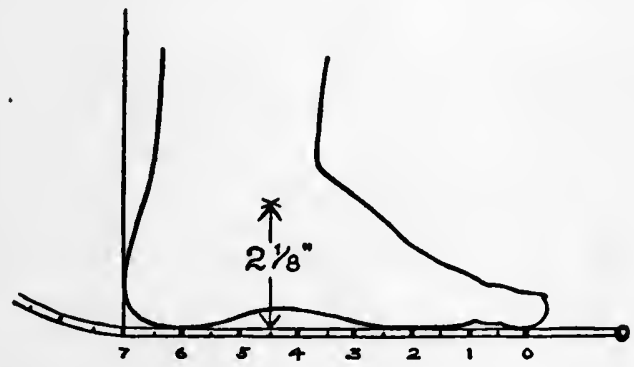

Weight-bearing: the arch is lowered as shown by the lowering of the tubercle of the scaphoid, and the foot is elongated as evidenced by the toe reaching beyond the 0 of the tape.

relation to the plane of the sole during rest and during weight-bearing. With this lowering of the dome there 
is a lengthening of the foot, which may be read on a tape measure by placing it under the weight-bearing foot, taking the length from heel to tip of great toe, and then with the foot still on the ground, removing the weight by having the subject sit. If instead of removing the weight, the subject rises on tip-toe, and the tape is brought up to the heel, it will be found that the foot has shortened.

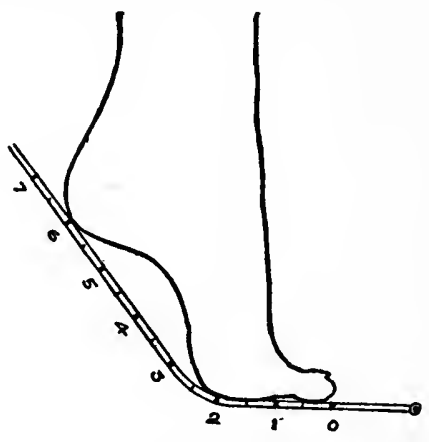

Fig. IOI. Alterations in the Length of the Foot During TipToeING

Tip-toeing: the foot is shortened as shown by reading of the tape, due to the elevation of the arch. The tubercle of the scaphoid is, of course, raised.

The slight flexion of the great-toe during rest and its position in activity, are also shown in these illustrations.

These changes in the length of the foot during rest, weight-bearing, and tip-toeing, are only what would be expected from the lowering and raising of the dome. A slipper which fits snugly the relaxed foot, will be too tight when standing and too loose when on tip-toe. A comfortable slipper usually flips-flaps up and down at the heel when used for walking; a slipper perfect for dancing is uncomfortable for walking. 


\section{INDEX}

Abductor minimi digiti, 37 . pollicis, 37 .

Abscesses, tuberculous, 240.

Accessorius, the, 33.

Adams, on "club-foot," 64 .

Adams on etiology of congenital club-foot, II5.

Adductor pollicis, 38 .

Allison's treatment of spastic paralysis, $18 \mathrm{I}$.

Ankle brace for tuberculous feet, 226.

Ankle-joint movement, I4, 43.

Apparatus for treating congenital club-foot, I30.

for treatment of infantile paralysis, 186.

Arch supporters, 92.

Arthrodesis of astragalo-scaphoid joints, I07.

Aspiration for gonorrheal infection of the foot, 246 .

Astragalo-scaphoid capsule, tenotomy of, I68.

Astragalectomy for congenital club-foot, 174 .

in paralysis of foot, 213.

Astragalus, the, 13-16.

Beely shoe-heel, 88 .

Berg on causes of congenital club-foot, II6.

Bessel-Hagen on frequency of club-foot, II3.

Bier's treatment of tuberculous disease of the foot, 23 I.
Bone operations for congenital club-foot, 172.

Bones, changes in, in talipes equino-varus, 118.

of the foot, 13 .

Bonnet's classification of clubfoot, II2.

Brace, Taylor's, 140.

Braces for congenital club-foot, I3I.

for foot paralysis, 206.

for hallux valgus, 259.

for tuberculous feet, 226.

for weak-foot, 94-96.

Broadhurst on tenotomy, I7I.

Broca on astragalectomy, 175.

Calcaneo-astragaloid joint, movements at, 45.

Calcaneo-scaphoid ligament, 23.

Calcaneum, the, I7.

Calf muscles, 24 .

Callosities, 275.

Campbell long leg brace, 227.

Cavus, accompanying infantile paralysis, I95.

Chilblains, 265.

Clavus, 268.

Claw-foot, Ig6.

Club-foot, Bonnet's classification, II2.

causes, bone theory, II5.

characteristics, etiology and treatment, 64-73.

congenital, II I-I77.

causes, germ theory, II5. 


\section{Club-foot-Continued.}

causes, nerve theory, Ir5. causes, pressure theory, II5. causes, theory of abnormal fetal movements, ir6. etiology of, II4. frequency of, II 3 . non-deforming, 64-I IO. pathology after use, I2I.

Congelation, 266.

Congenital club-foot, adhesive plaster for, I3I. astragalectomy for, 174 . bone operations for, 174 . braces for, I3I.

felt splints for, 133 . muslin bandages for, 130 . pathology after use, I2I. theories of causes, II4. varieties, III. wood splint for, $\mathrm{I} 36$.

Congenital deformities, II3. in the tibia and femur, I43.

Coote, Holmes, on "Joint Disease," 64.

Corns, 268.

Cuboid, the, I7.

Cuneiform bones, the, I8.

Davis's physiological law, 157 .

Detmould on subcutaneous tenotomy, i6r.

Diagnosis of case, 59-63. of tuberculous foot, 217 . of weak-foot, 79 .

Diet, for tuberculous foot, 238 . Differential diagnosis of tuberculous foot, 223 .

Dorsal flexion at ankle joint, 43 . Douching in post-operative treatment, 108 .

Duval, E., on tenotomy, I7I

Early treatment of congenital club-foot, 125.
Erythromelalgia, 268.

Etiology of congenital club-foot, I 4 .

Eschricht on causes of congenital club-foot, II6.

Exercises for flat-foot, 89.

Extensor brevis digitorum, 39. longus digitorum, 36. proprius pollicis, 36 .

External lateral ligament, 22.

Fallen arch, 251.

Felt splint for congenital clubfoot, 133 .

Flat-foot-arthrodesis operation, 107.

diagnosis, 83 .

osseous, 84.

pathology of, 85 .

Plaster of Paris dressing for, I03.

post-operative treatment, 108. tarsectomy for, 10\%.

tenotomy for, 106.

treatment of, 86.

Flexor brevis digitorum, 33, 37 . brevis pollicis, 38 .

longus digitorum, 32 .

longus digitorum, tenotomy of, I68.

longus pollicis, 28.

Foot at rest, 48.

bones of, I3.

ligaments of, 2 I.

muscles and tendons of, 23.

nerves of, 39 .

standing, 49.

Frequency of club-foot, II3.

Frost-bite, 266.

Galen on causes of congenital club-foot, I 16.

Gastrocnemius, contraction of, 65. 
Golding-Bird, on hollow-foot, 196.

Golding-Bird's scaphoidectomy, 108.

Gonorrheal infection of foot, 243 .

Great-toe joint, 48.

Guerin, J., on etiology of congenital club-foot, II5.

Hallux valgus, 257.

Hammer-toe, 26I.

Heel-and-toe walking, 55 .

Heel-cord, shortened, treatment, 250.

Heliotherapy, 235 .

Hippocrates on causes of congenital club-foot, $\mathrm{I} 16$.

History of case, 59.

Hoffa on causes of congenital club-foot, II6.

Hollow claw-foot, 196.

Hyperidrosis, 266.

Infantile paralysis, treatment, 202.

Ingrowing toe-nail, 272.

Injections for tuberculous joints, 242.

Inspection of patient, 59.

Intermittent limping, 264.

Internal lateral ligament, 22.

Interossei, the, 38 .

Ketch on congenital deformities, II3.

Kirmisson on frequency of clubfoot, II3.

on injections for tuberculous joints, 242.

on treatment of congenital club-foot, $\mathbf{I} 46$.

Kocker on causes of congenital club-foot, 116 .

Lannelongue on frequency of club-foot, II3.
Lateral traction shoe, 203.

Lechiberder on congenital deformities, I13.

Ligaments, changes in, in talipes equino-varus, 120 .

of the foot, $2 \mathrm{r}$.

Liniments, 109.

Little on etiology of congenital club-foot, II5.

on subcutaneous tenotomy, $\mathbf{1 6 1}$.

Lumbricales, the, 33.

Lund, on astragalectomy, 174 .

Manipulations for weak-foot and flat-foot, 97.

Manipulative treatment of congenital club-foot, 126.

Mayo, C. H., on treatment of hallux valgus, 261 .

Medio-tarsal joint, movements at, 46.

Mensuration of tuberculous foot, 222.

Metatarsal bones, the, 18 . joints, manipulation of, ror.

Metatarsalgia, 25I.

Morton's toe, 253.

Movements of the foot, 43.

Murphy, J. K., on congenital deformities of the tibia and femur, 143.

Muscles, changes in, in talipes equino-varus, 120.

of the calf, 24.

of the foot, 23 .

Muscular spasms in tuberculous foot, 221 .

Myasthenia angio-sclerotica, 264.

Myers wrench, 154.

Nerves of the foot, 39 .

Neurotomy for spastic paralysis, 180.

Nutt's traction shoe, 73 . 
Ogston's operation-arthrodesis of the astragalo-scaphoid joint, 107, 176.

Onychia, 272.

Operation for hallux valgus, 260 . hammer-toe, 262. shortened heel-cord, 250. tuberculous foot, 238 .

Ogston's arthrodesis, 107. tenotomy for flat-foot, 106. tenotomy of tendo Achillis, 76 .

Operative treatment of congenital club-foot, 153 .

Os calcis, the, 17.

Osseous flat-foot, 84 .

Osteoclasis for congenital clubfoot, 144 .

Osteotomies, multiple cuneiform, I76.

Osteotomy for congenital clubfoot, 144 .

Pain, significance of in diagnosis, 62.

Painful heel, 248.

soles, 276.

Palpation, 6I.

Paralysis, infantile, 183-216. of plantar muscles, 198 . of tibialis anticus, 201. residual, 183 spastic, 178 .

Allison's treatment, $18 \mathrm{I}$.

Schwab's treatment, I8I.

Stoeffel's treatment, I8I.

treatment, I80.

Paralytic talipes calcaneus, 193. equinus, 192.

valgus, $19 \mathrm{I}$.

varus, IgI.

Pare on causes of congenital club-foot, I 16 .

Parker on causes of congenital club-foot, I 16 .
Parker on tenotomy of the astragalo-scaphoid capsule, 168.

Paronychia, 272.

Peroneal muscles, 27.

Pernio (chilblains), 265.

Peroneus brevis, tenotomy of, 168.

longus, tenotomy of, I68. tertius, 36 .

Phalanges, the, 18.

Phelps' operation for congenital club-foot, 172.

Physiological laws, Wolff's and Davis', 157.

Plantar fascia, 21.

fascia, tenotomy of, 169 .

flexion, 30-32. of the foot, 43 . ligaments, 2I. muscles, paralysis of, 198 . neuralgia, 268.

Plaster-of-Paris dressing for flat-foot, 103 .

for congenital club-foot, I37.

Policeman's heel, 248.

Post-operative treatment of flatfoot, 108.

Posterior ligament, 23.

Pott's disease, complicated by paralysis, 178 .

Radiography of tuberculous foot, 222.

Raynaud's disease, 262.

Residual paralysis, 183 .

Scaphoid, the, 17 .

Scaphoidectomy for flat-foot, 108 .

Scarpa on catuses of congenital club-foot, 116.

Schwab's treatment of spastic paralysis, I8I.

Sea-bathing for tuberculous foot, 236. 
Sesamoid bones, the, 18.

Shattock on causes of congenital club-foot, in 6 .

Shaffer's brace, 96 .

Shaffer's foot, 65-77. etiology, 72 .

characteristics, $65-67$. symptoms, 68 . treatment, 72 .

Shaffer's liniment, IIo. traction shoes, 72, 202.

Shoe, lateral traction, 203. Nutt's traction, 73.

Shaffer's traction, 72 . the Beely-heel, 88. Willard's, 139.

Shoes, the heels, 280 . importance of proper fitting, 284.

the toes, 280 .

the uppers, 283 .

the shank, 281.

the rocker-sole, 284 .

Silk ligaments, use of in paralysis of foot, 212 .

Sinuses, tuberculous, 240.

Skeleton of the foot, 18 .

Soles, painful, 276.

Spastic paralysis, 178.

Standing, proper position, 57 .

Stockings, 277.

Stoeffel's treatment of spastic paralysis, I8I.

Strapping weak-foot with adhesive plaster, 100.

Stromeyer on subcutaneous tenotomy, $16 r$.

Sub-astragaloid joint, movements at, 45 .

Subcutaneous tenotomy, I61.

Sun-bath for tuberculous foot, 235.

Sweating feet, 266.

Symptoms of Shaffer's foot, 68.
Talipes calcaneo-valgus, definition of, III.

calcaneo-varus, definition of, III.

calcaneus, congenital, treatment, I5I.

definition of, III.

paralytic, I93.

cavus, congenital, I53.

comparative frequency of varieties, II4.

equino-cavus, congenital, 153 .

equino-valgus, congenital, treatment, 150.

definition of, II r.

equino-varus, changes in bones in, 118.

changes in muscles and ligaments in, 120.

confirmation of structural changes in, 123.

definition of, III.

early treatment, 125-I45. . general appearance, II6.

pathological changes in, $\mathbf{I} 48$. treatment when child begins to stand, $146-150$.

equinus, congenital, treatment, 150.

definition of, IIr.

paralytic, 192.

planus, congenital, 153 .

varus, congenital, treatment, I5I.

definition of, III. paralytic, $19 \mathrm{I}$.

valgo-cavus, congenital, 153 .

valgus, congenital, treatment, 150.

valgus and varus, congenital, treatment, 152.

valgus, definition of, III. paralytic, 191. 
Tamplin on frequency of clubfoot, Ir3.

Tarsectomy, cuneiform, I76. for flat-foot, 107.

Taylor's club-foot brace, I40.

Tendo Achillis, 26. contraction of, 64 . tenotomy of, 76, 163 . transplantation, 209.

Tendons of the foot, 23.

Tenotomy for flat-foot, 106 . for spastic paralysis, 180 . indications for, 170 .

of astragalo-scaphoid capsule, I68.

of flexor longus digitorum, I68. of peroneus longus and brevis, 168.

of plantar fascia, 169 .

of tendo Achillis, 76, 163.

of tibialis anticus, $\mathrm{I} 68$.

of tibialis posticus, 166 . subcutaneous, I6I.

Therapeutics of early treatment of congenital club-foot, 145 .

Thomas knee brace, 229. wrench, 154 .

Tibialis anticus, 35 . paralysis of, 201 . tenotomy of, 168. posticus, 34 . tenotomy of, 166.

Toe-walking, 55 .

Traction shoes, Shaffer's, 72.

Transversus pedis, the, 38 .

Treatment, Bier's, for tuberculous foot, 231 . constitutional, for tuberculous foot, 237.

callosities, 275 .

chilblains, 265 .

congenital club-foot, I25-I 77 .

early, 125 .

manipulation, $\mathbf{1 2 6}$.
Treatment, congenital club-foot apparatus, 130.

muslin bandages, 130 .

adhesive plaster, $13 \mathrm{I}$.

braces, 131 .

felt splint, 133 .

wood splint, 136 .

plaster-of-Paris, 137 .

Willard's shoe, I39.

Taylor's brace, 140.

osteotomy, 144.

osteoclasis, I44.

therapeutics of, 145 .

when child begins to stand, 146.

operative procedure, I53-172. wrenching, 153.

tenotomy, 161-172. indications for, 170.

Phelps' operation, 172.

bone operations, 172 .

astragalectomy, I74.

Ogston's operation, 176 .

cuneiform tarsectomy, 176 .

multiple cuneiform osteotomies, 176

congenital talipes calcaneus, I5I.

congenital talipes equino-valgus, 150.

congenital talipes equinus, 150 . congenital talipes valgus, $\mathbf{I} 50$. congenital talipes varus, I5I. corns, 270.

fallen arch, 253.

flat-foot, 86 .

frost-bite, 266.

hallux valgus, 257 .

hammer-toe, 26r.

gonorrheal infection of the foot, 245 .

infantile paralysis, 202.

instrumental stretching, 202.

lateral traction shoe, 203. 
Treatment of infantile paralysis braces, 206.

wedge sole, $2 n 9$.

tendon transplantation, 209.

silk ligaments, 212.

astragalectomy, 213 .

ingrowing toe-nail, 273.

intermittent limping, 264.

metatarsalgia, 253.

Morton's toe, 256 .

painful heel, 249.

perforating ulcer of the foot, 264.

Raynaud's disease, 263.

Shaffer's foot, 72.

shortened heel-cord, 250.

sinuses, 240.

spastic paralysis, 179.

sweating feet, 267.

tuberculous abscesses, 240.

tuberculous disease of the foot, 225.

tuberculous joints, 242.

tuberculin, for tuberculous foot, 233 .

weak-foot, 86.

Tubby on astragalectomy, 174 .

Tubby, on talipes equino-varus, 148.

Tuberculin test for tuberculous foot, 223.

Tuberculin treatment for tuberculous disease of the foot, 233 .

Tuberculous foot, diagnosis, 217. mensuration, 222.
Tuberculous foot-Continued. muscular spasms in, 220. primary focus, 217 . joints, 242.

Ulcers of the foot, 264 .

Vaccine treatment for gonorrheal infection of the foot, 246 .

Volkman, on causes of congenital club-foot, II6.

Walking, 54.

Weak-foot, adhesive plaster strapping, 100.

characteristics, 78 .

diagnosis, 79.

exercises for, 89.

manipulations for, 97 .

treatment, 83 .

Wedge sole, 209.

Weight, distribution of on the feet, $4 \mathrm{r}$.

Whitman on congenital deformities, I13.

Whitman's astragalectomy, 213. brace, 94 .

Willard, on early treatment of club-foot, 125 .

Willard's shoe, 139 .

Wolff's physiological law, I57.

Wood splint for congenital clubfoot, 136 .

Work, rest and fatigue, $5 \mathrm{I}$.

Wrenching in operative treatment of club-foot, 153 . 



\section{Practical Monographs}

DISEASES AND DEFORMITIES OF THE FOOT

By John Joseph NUTT, M.D., Orthopedic Surgeon, Willard Parker Hospital, New York, etc. 8 vo, over 300 pages, 105 illustrations, prepaid...\$2.75

This treatise is prepared for the use of those physicians who have not had the time or the opportunity for thorough study of this of ten neglected subject and who feel keenly their inability to prescribe scientifically and successfully for the many who consult them regarding their pedal conditions. The illustrations will be found an especially valuable feature.

\section{DIAGNOSIS OF NERVOUS DISEASES}

By Purves Stewart, M.D., F.R.C.P. Third Edition. 8vo, 500 pages, 225 illustrations and colored plates, cloth, prepaid............\$4.50.

"The plainness of the text, and the directness of approaching symptoms, the clear-cut lines to accurate methods, all combine to render this work a dependable ally of the general practician, who, as a rule, devotes too scant attention to nervous disorders."-Medical World.

\section{DISEASES OF THE RECTUM AND} PELVIC COLON

By Martin Bodkin, M.D., Rectal Surgeon, St. Mary's Hospital, New York. 8vo, 425 pages, 90 special illustrations, cloth, prepaid.......\$3.50

The author's purpose has been to condense the results of his own practice and the labors of other specialists into a book that covers the entire field in the most practical manner and not to burden the reader with an exhaustive and unnecessarily technical treatise.

\section{NEW ASPECTS OF DIABETES}

By Prof. CARI, VaN Noorden, Vienna. 8vo, diagrams and tables, cloth, prepaid .......\$1.50

The author's experience, during the past seven years, with over 2,000 cases of diabetes in his clinic and sanatorium, forms the basis of these American lectures.

\section{E. B. TREAT \& CO., Medical Publishers} 241-243 Weat 23d Strect :: $\quad$ is NEW YORK 





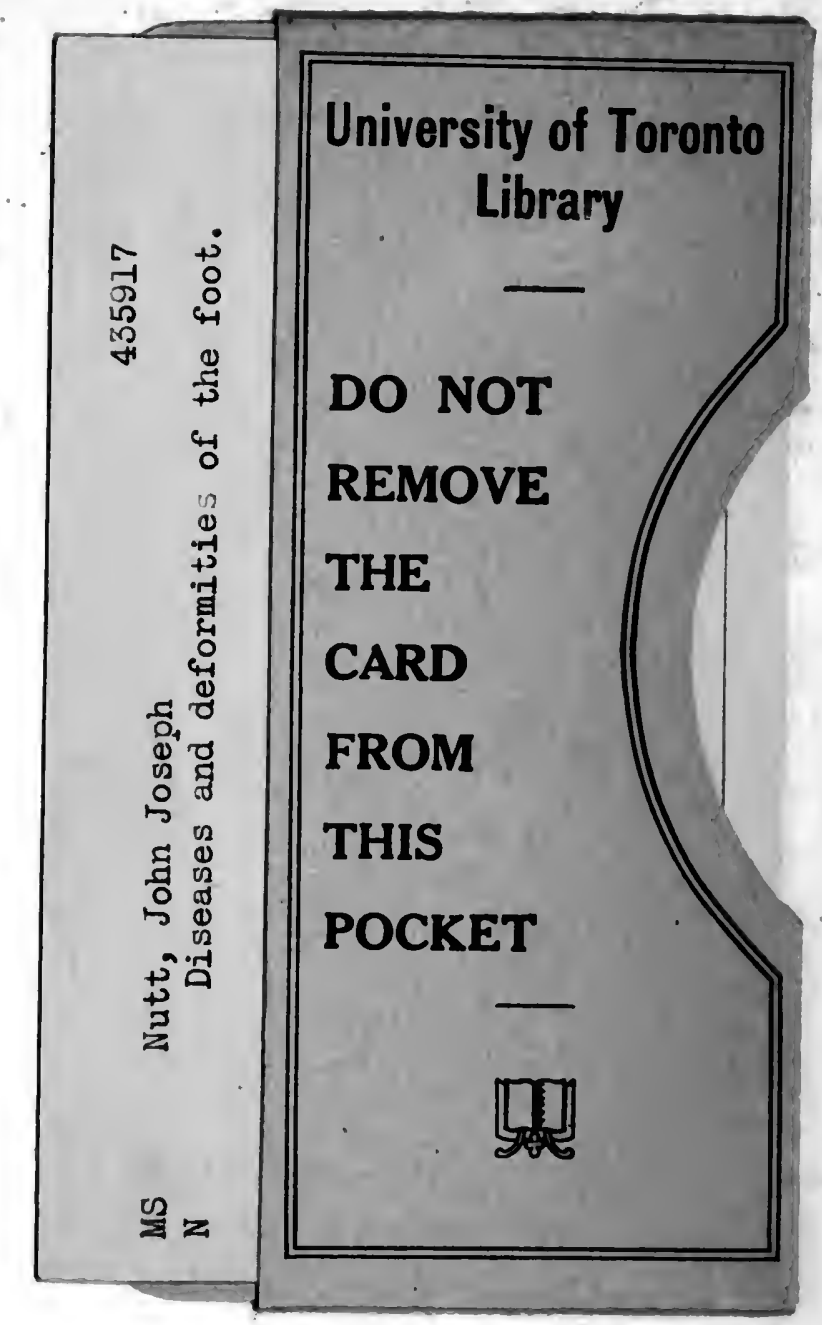


